\title{
Um estudo dos modelos polinomiais com erros nas variáveis na função de verossimilhança corrigida e escore corrigido
}

Arturo Alejandro Zavala Zavala

\author{
Tese apresentada \\ ao \\ Instituto de Matemática e estatística \\ da \\ Universidade de São Paulo \\ para \\ obtenção do grau \\ de \\ Doutor em Estatística
}

Área de Concentração: Estatística Orientador: Prof. Dr. Heleno Bolfarine

(i) Este trabalho contou parcialmente com apoio financeiro do CNPq. São Paulo - Maio - 2004 


\title{
Um estudo dos modelos polinomianis com erros nas variåveis na frumção de verossimillhauça corrigida e escore corrigido
}

\author{
Este exemplar corresponde à redação final \\ da tese de doutorado devidamente \\ corrigida e defendida por \\ Arturo Alejandro Zavala Zavala \\ e aprovada pela comissão julgadora.
}

São Paulo, Maio de 2004.

Banca examinadora:

Prof. Dr. Heleno Bolfarine (Orientador) - IME-USP

Prof. Dr. Gilberto Alvarenga Paula - IME-USP

Prof. Dra. Pilar Iglesias - UNIV.C.CHILE

Prof. Dr. Filidor Vilca Labra - IME-UNICAMP

Prof. Dr. Mário de Castro Andrade Filho - ICMC-USP 
谄 A meus grandes amigos Vicente Garibay Cancho e Fernando Carvalho Trindade para eles todo meu reconhecimento.

谄 A todos os peruanos presentes e em especial aos formados na Universidad Nacional Agraria La Molina.

赗 A meus colegas de trabalho e alunos da Universidade Estadual de Mato Grosso - Barra do Bugres - UNEMAT, muito grato pelo apoio e ajuda nesta nova etapa de minha vida profissional.

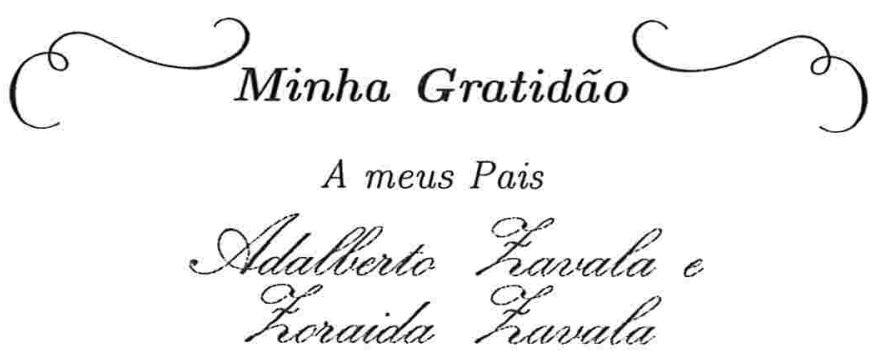

A minha querida esposa

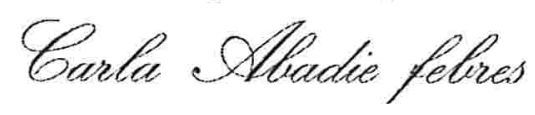

A meus lindos filhos

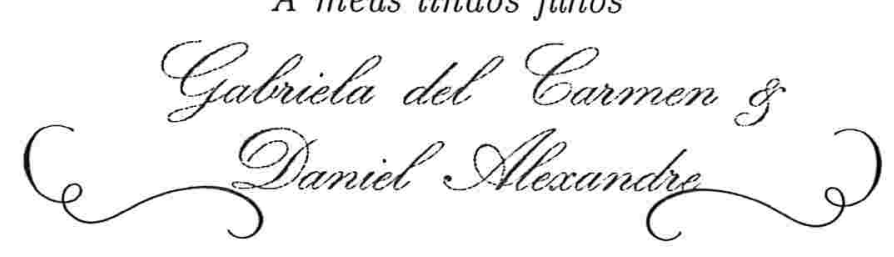




\section{RESUMO}

Neste trabalho, estudamos os modelos polinomiais com erros nas variáveis considerando as metodológicas da função de verossimilhança corrigida desenvolvida por Nakamura (1990) e a função escore corrigido desenvolvida por Bolfarine e Gimenez (1997).

Exibimos os casos quando o modelo com erros nas variáveis tem um erro aditivo, assim quando este erro é multiplicativo, além disso consideramos os dois possíveis supostos, isto é, quando o modelo é funcional e quando o modelo é estrutural, logo fazemos uma generalização para modelos multivariados, considerando neste caso os modelos de calibração comparativa.

Nos casos onde a função de verossimilhança corrigida exista num modelo aditivo, será considerada um estudo de Influência Local. Consideramos exemplos de Simulação de Monte Carlo e exemplos concretos para cada situação. 


\section{ABSTRACT}

In this work, we study polynomials models with errors in variables, using methodologies based of the corrected likelihood function development by Nakamura (1990) and the corrected score function development by Bolfarine and Gimenez (1997).

We study the cases when the models present additive errors in variables, and also when they have multiplicative errors. For one hand, two situations are considered, seat is, functional models and estrutural models. The models are also generalized, considering multivariate models, using comparative calibrations models.

In this case since the likelihood function corrected the methodology of the local influence is used for studying influence of observations on the estimate parameters. 
Resumo

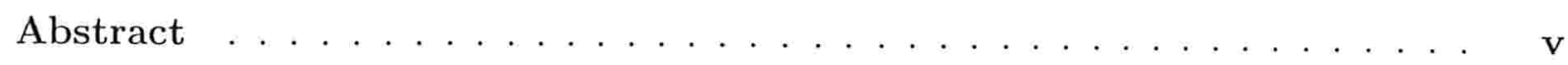

1 Apresentação do Problema. . . . . . . . . . . . . . . . 1

1.1 Introdução. . . . . . . . . . . . . . . . . . . . 1

1.2 Alguns trabalhos em modelos polinomiais com erros nas variáveis. . . . . . . 3

1.3 O método para a correção de viés em modelos com erros nas variáveis. . . . 10

1.4 Influência Local . . . . . . . . . . . . . . . . . . . . . . . . . . . . . . 13

2 Modelo Aditivo . . . . . . . . . . . . . . . . . . . . 16

2.1 Introdução. . . . . . . . . . . . . . . . . . . . . 16

2.2 Caso $1: \sigma_{u}^{2}$ conhecida . . . . . . . . . . . . . . . 17

2.2 .1 Estimação dos parâmetros . . . . . . . . . . . . . . . 17

2.2 .2 Obtenção da matriz de covariâncias assintóticas . . . . . . . . . . . 32

2.2 .3 Teste de hipóteses para $\beta \ldots \ldots \ldots \ldots \ldots$

2.2 .4 Aplicações numéricas. . . . . . . . . . . . . . . . . 35

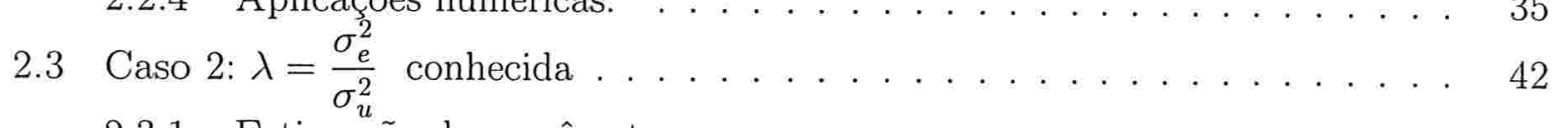

2.3.1 Estimação dos parâmetros . . . . . . . . . . . . . . . . . 42

2.3.2 Obtenção da matriz de covariâncias assintóticas . . . . . . . . . . . . 47

2.3 .3 Teste de Hipótese. . . . . . . . . . . . . . . . . . . . . . . . . . 47 
2.3.4 Aplicações numéricas . . . . . . . . . . . . . . . . . . . . . . . . 48

2.4 Influência Local . . . . . . . . . . . . . . . . . . . . . . . . . . . 53

2.4 Metodologia . . . . . . . . . . . . . . . . 53

2.4 .2 Esquemas de perturbação . . . . . . . . . . . . . . . 55

2.4 .3 Aplicação . . . . . . . . . . . . . . . . . . . 60

2.5 Caso 3: Variâncias heterogêneas . . . . . . . . . . . . . . . . . . . 67

2.5.1 Metodologia ...................... 67

2.5 .2 Exemplos . . . . . . . . . . . . . . . . . . 68

2.5.3 Obtenção da matriz de covariâncias Assintóticas . . . . . . . . . . . 72

2.5 .4 Teste de hipóteses para $\beta \quad \ldots \ldots \ldots$. . . . . . . . . . 72

2.5 .5 Aplicações numéricas . . . . . . . . . . . . . . . . . . . 73

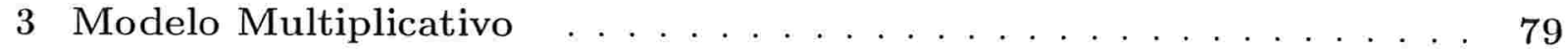

3.1 Introdução . . . . . . . . . . . . . . . . . . . . . . . . . . . . . . . 79

3.2 Caso $1: \sigma_{u}^{2}$ conhecida . . . . . . . . . . . . . . . . 80

3.2 .1 Estimação dos parâmetros . . . . . . . . . . . . . . . 80

3.2 .2 Exemplos . . . . . . . . . . . . . . . . . . . . . 83

3.2.3 Obtenção da matriz de covariâncias assintóticas . . . . . . . . . . 85

3.2 .4 Teste de hipóteses para $\beta \ldots \ldots \ldots \ldots$. . . . . . . . 86

3.2 .5 Aplicação numérica . . . . . . . . . . . . . . . . . . . 88

3.3 Caso $2: \lambda=\frac{\sigma_{e}^{2}}{\sigma_{u}^{2}}$ conhecida . . . . . . . . . . . . . . . . . . . 92

3.3 .1 Estimação dos parâmetros . . . . . . . . . . . . . . . . . . 92

3.3 .2 Exemplos . . . . . . . . . . . . . . . . . . . 93

3.3 .3 Aplicações numéricas . . . . . . . . . . . . . . . . . . . . . 97

3.4 Caso 3: Variâncias heterogêneas . . . . . . . . . . . . . . . . . . . 100

3.4 .1 Estimação dos parâmetros . . . . . . . . . . . . . . . . . . 100

3.4 .2 Exemplos . . . . . . . . . . . . . . . . . . . 101

3.4 .3 Aplicações numéricas . . . . . . . . . . . . . . . . . . . . . . 103

4 Calibração Comparativa . . . . . . . . . . . . . . . . . 108

4.1 Introdução . . . . . . . . . . . . . . . . . . . . . . . 108

4.2 Variâncias homogêneas . . . . . . . . . . . . . . . . . . . . . 110 
4.2.1 Caso 1: $\sigma_{u}^{2}$ conhecida . . . . . . . . . . . . . . . . . 110

4.3 Caso 2: $\lambda=\frac{\sigma_{e}^{2}}{\sigma_{u}^{2}}$ conhecida . . . . . . . . . . . . . . . . . . 119

4.4 Variâncias heterogêneas . . . . . . . . . . . . . . . . . . 127

4.4.1 Caso 1: $\sigma_{u}^{2}$ conhecida . . . . . . . . . . . . . . . . . 127

4.5 Caso 2: $\lambda=\frac{\sigma_{e}^{2}}{\sigma_{u}^{2}}$ conhecida . . . . . . . . . . . . . 135

5 Considerações finais f. . . . . . . . . . . . . . . . 143

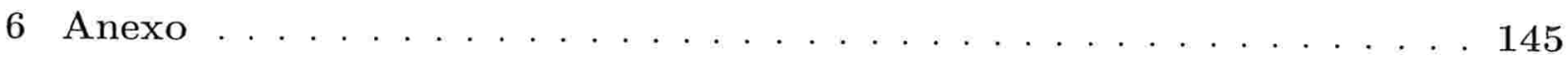

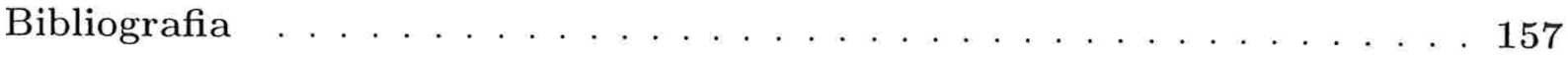




\section{LISTA DE TABELAS}

2.1 Estimativas segundo os métodos "naive"e "escore corrigido"para diferentes tamanhos de amostra. . . . . . . . . . . . . . . . 36

2.2 Comparação das estimativas segundo os métodos "naive", "escore corrigido"e de Fuller . . . . . . . . . . . . . . . . . . . . . . . . . . . . . . . . . 39

2.3 Teste de Hipótese para os parâmetros do modelo. . . . . . . . . . . . . . . 40

2.4 Estimativas segundo os métodos "naive"e "escore corrigido" para diferentes tamanhos de amostra. . . . . . . . . . . . . . . . . . . . 49

2.5 Estimativas segundo as metodologias "naive" e "escore corrigido". . . . . . 52

2.6 Avaliação das estimativas do método "escore corrigido", usando o teste de wald. 53

2.7 Índice de variação percentual nos parâmetros em um modelo quadrático devido aos pontos $1,2,3$ e $4 \ldots \ldots \ldots 66$

2.8 Estimativas segundo o método "naive" para diferentes tamanhos de amostra em um modelo de regressão quadrática quando são consideradas variâncias heterogêneas. . . . . . . . . . . . . . . . . . . 75

2.9 Estimativas e testes de hipóteses para os parâmetros de um modelo quadrático 78

3.1 Estimativas segundo os métodos "naive"e "escore corrigido"para diferentes tamanhos de amostra. . . . . . . . . . . . . . . . . . . . . 89

3.2 Estimativas segundo o método de escore corrigido . . . . . . . . . . . . . . 91 
3.3 Estimativas segundo os métodos "naive"e "escore corrigido" para diferentes tamanhos de amostra. . . . . . . . . . . . . . . . . . . 98

3.4 Estimativas segundo o método de escore corrigido . . . . . . . . . . . . . 100

3.5 Estimativas segundo os métodos "naive"e "escore corrigido" para diferentes tamanhos de amostra. . . . . . . . . . . . . . . . . 105

3.6 Estimativas segundo o método de escore corrigido . . . . . . . . . . . . . 107

4.1 Estimativas dos parâmetros segundo o método "naive" para diferentes tamanhos de amostra e Instrumento 1. . . . . . . . . . . . . . . . . 113

4.2 Estimativas dos parâmetros segundo o método "escore corrigido"para diferentes tamanhos de amostra e Instrumento 1. . . . . . . . . . . . . . . . . 113

4.3 Estimativas dos parâmetros segundo o método "naive" para diferentes tamanhos de amostra e Instrumento 2 . . . . . . . . . . . . . . . . . . 115

4.4 Estimativas dos parâmetros segundo o método "escore corrigido"para diferentes tamanhos de amostra e Instrumento 2 . . . . . . . . . . . . . . . 115

4.5 Estimativas dos parâmetros segundo o método "naive" para diferentes tamanhos de amostra e Instrumento 3 . . . . . . . . . . . . . . . 117

4.6 Estimativas dos parâmetros segundo o método "escore corrigido"para diferentes tamanhos de amostra e Instrumento 3 . . . . . . . . . . . . . 117

4.7 Estimativas dos parâmetros segundo o método "Naive" para diferentes tamanhos de amostra e Instrumento 1. . . . . . . . . . . . . . . . . 121

4.8 Estimativas dos parâmetros segundo o método "escore corrigido"para diferentes tamanhos de amostra e Instrumento 1 . . . . . . . . . . . . . . . 121

4.9 Estimativas dos parâmetros segundo o método "naive" para diferentes tamanhos de amostra e Instrumento 2 . . . . . . . . . . . . . . . . . . 123

4.10 Estimativas dos parâmetros segundo o método "escore corrigido"para diferentes tamanhos de amostra e Instrumento 2 . . . . . . . . . . . . . . 123

4.11 Estimativas dos parâmetros segundo o método "naive" para diferentes tamanhos de amostra e Instrumento 3 . . . . . . . . . . . . . . . . . 125

4.12 Estimativas dos parâmetros segundo o método "escore corrigido"para diferentes tamanhos de amostra e Instrumento 3. . . . . . . . . . . . . . . . 125 
4.13 Estimativas dos parâmetros segundo o modelo "naive" para diferentes tamanhos de amostra e Instrumento 1. . . . . . . . . . . . . . . . . . . . 129

4.14 Estimativas dos parâmetros segundo o modelo "escore corrigido"para diferentes tamanhos de amostra e Instrumento 1 . . . . . . . . . . . . . . . 130

4.15 Estimativas dos parâmetros segundo o modelo "naive" para diferentes tamanhos de amostra e Instrumento 2 . . . . . . . . . . . . . . . . . . . . . 131

4.16 Estimativas dos parâmetros segundo o modelo "escore corrigido"para diferentes tamanhos de amostra e Instrumento 2 . . . . . . . . . . . . . . . . 131

4.17 Estimativas dos parâmetros segundo o modelo "naive" para diferentes tamanhos de amostra e Instrumento 3 . . . . . . . . . . . . . . . . . . . 133

4.18 Estimativas dos parâmetros segundo o modelo "escore corrigido"para diferentes tamanhos de amostra e Instrumento 3 . . . . . . . . . . . . . . . . 133

4.19 Estimativas dos parâmetros segundo o modelo "naive" para diferentes tamanhos de amostra e Instrumento 1 . . . . . . . . . . . . . . . . 137

4.20 Estimativas dos parâmetros segundo o modelo "escore corrigido"para diferentes tamanhos de amostra e Instrumento 1 . . . . . . . . . . . . . . . . 137

4.21 Estimativas dos parâmetros segundo o modelo "naive" para diferentes tamanhos de amostra e Instrumento $2 \ldots \ldots$. . . . . . . . . . . . . . . . 139

4.22 Estimativas dos parâmetros segundo o modelo "escore corrigido"para diferentes tamanhos de amostra e Instrumento 2 . . . . . . . . . . . . . . 139

4.23 Estimativas dos parâmetros segundo o modelo "naive" para diferentes tamanhos de amostra e Instrumento 3. . . . . . . . . . . . . . . . . . . 141

4.24 Estimativas dos parâmetros segundo o modelo "escore corrigido"para diferentes tamanhos de amostra e Instrumento 3. . . . . . . . . . . . . . . . . 141

6.1 Dados simulados por Fuller (1987) para um polinômio de segundo grau. . . 145

6.2 Dados simulados por Fuller (1987) para um polinômio de segundo grau (Continuação...) . . . . . . . . . . . . . . . . . . . 146

6.3 Dados do exemplo 4 em Galea-Rojas et. al. (2003). . . . . . . . . . . . . 147

6.4 Dados do exemplo 4 em Galea-Rojas et. al. (2003). . . . . . . . . . . . . 148

6.5 Dados do exemplo 4 em Galea-Rojas et. al. (2003). . . . . . . . . . . . . 149 
6.6 Dados do exemplo 4 em Galea-Rojas et. al. (2003). . . . . . . . . . . 150

6.7 Dados do exemplo 4 em Galea-Rojas et. al. (2003). . . . . . . . . . . . . 151

6.8 Dados do exemplo 4 em Galea-Rojas et. al. (2003). . . . . . . . . . . . . 152

6.9 Dados do exemplo 4 em Galea-Rojas et. al. (2003). . . . . . . . . . . . . 153

6.10 Dados do exemplo 4 em Galea-Rojas et. al. (2003). . . . . . . . . . . . . 154

6.11 Dados do exemplo 4 em Galea-Rojas et. al. (2003). . . . . . . . . . . . 155

6.12 Dados do exemplo 4 em Galea-Rojas et. al. (2003). . . . . . . . . . . . 156 


\section{LISTA DE FIGURAS}

2.1 Comportamento das estimativas "naive"e "escore corrigido" segundo o tamanho da amostra . . . . . . . . . . . . . . . . . . 37

2.2 Gráfico da dispersão das variáveis $Y_{1}$ e $W \ldots \ldots \ldots \ldots$

2.3 Comportamento das estimativas dos três modelos . . . . . . . . . . . . . . 39

2.4 Comportamento das estimativas do modelo linear e quadrático no escore corrigido. . . . . . . . . . . . . . . . . . . 41

2.5 Comportamento das estimativas "naive" e "escore corrigido"segundo o tamanho

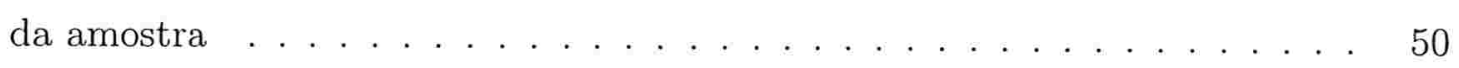

2.6 Gráfico da dispersão das variáveis $Y_{2}$ e $W \ldots \ldots \ldots$. . . . . . . . 51

2.7 Gráfico do comportamento das estimativas dos métodos "naive"e "escore corrigido"

2.8 Gráfico de índice segundo o esquema de perturbação da ponderação dos casos 63

2.9 Gráfico de índice segundo o esquema de perturbação da variável resposta . . 64

2.10 Gráfico de índice segundo o esquema perturbação das variâncias dos erros de medição da variável resposta . . . . . . . . . . . . . . . . . . . 64

2.11 Gráfico de índice segundo o esquema de perturbação da covariável . . . . . . 65

2.12 Comportamento das estimativas "naive" e "escore corrigido" segundo o tamanho da amostra . . . . . . . . . . . . . 76

2.13 Gráfico de dispersão dos dados . . . . . . . . . . . . . . . . . . . 77

2.14 Gráfico de dois modelos propostos, Linear e Quadrático . . . . . . . . . . . 78 
3.1 Comportamento das estimativas "naive"e "escore corrigido" segundo o tamanho

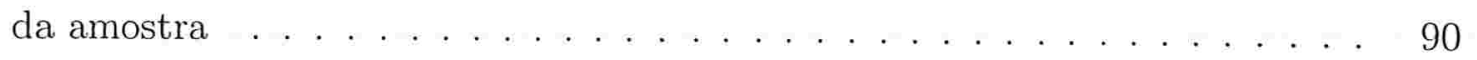

3.2 Comportamento das estimativas "naive"e "escore corrigido" segundo o tamanho da amostra . . . . . . . . . . . . . . . . . . .

3.3 Comportamento das estimativas "naive"e "escore corrigido" segundo o tamanho da amostra

3.4 Gráfico comparativo dos modelos aditivo e multiplicativo na metodologia escore corrigido

4.1 Comportamento das estimativas "naive"e "escore corrigido" segundo o tamanho da amostra para o instrumento 1

4.2 Comportamento das estimativas "naive"e "escore corrigido" segundo o tamanho da amostra e instrumento 2

4.3 Comportamento das estimativas "naive"e "escore corrigido" segundo o tamanho da amostra

4.4 Comportamento das estimativas "naive"e "escore corrigido" segundo o tamanho da amostra no instrumento $1 \ldots \ldots$. . . . . . . . . . . . . . 122

4.5 Comportamento das estimativas "naive"e "escore corrigido" segundo o tamanho da amostra no instrumento 2 . . . . . . . . . . . . . . . . . . . 124

4.6 Comportamento das estimativas "naive"e "escore corrigido" segundo o tamanho da amostra no instrumento $3 \ldots \ldots \ldots \ldots \ldots$

4.7 Comportamento das estimativas "naive"e "escore corrigido" segundo o tamanho da amostra no instrumento $1 \ldots \ldots$. . . . . . . . . . . . . 130

4.8 Comportamento das estimativas "naive"e "escore corrigido" segundo o tamanho da amostra no instrumento $2 \ldots \ldots$. . . . . . . . . . . . . . . 132

4.9 Comportamento das estimativas "naive"e "escore corrigido" segundo o tamanho da amostra no instrumento $3 \ldots \ldots$. . . . . . . . . . . . . . 134

4.10 Comportamento das estimativas "naive"e "escore corrigido" segundo o tamanho da amostra no instrumento $1 \ldots \ldots$. . . . . . . . . . . . . . . 138

4.11 Comportamento das estimativas "naive"e "escore corrigido" segundo o tamanho

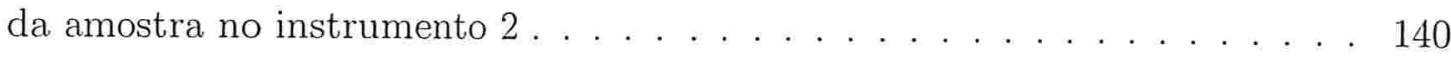


4.12 Comportamento das estimativas "naive"e "escore corrigido" segundo o tamanho da amostra no instrumento $3 \ldots \ldots$. . . . . . . . . . . . . . . . . 142 


\section{CAPÍTULO}

\section{Apresentação do Problema.}

\subsection{Introdução.}

Muitas áreas do conhecimento apresentam problemas envolvendo relações entre duas ou mais variáveis. Estas relações podem ser estudadas no âmbito dos modelos de regressão, podendo-se apresentar segundo o seguinte modelo

$$
Y_{i}=f_{i}\left(X_{i} ; \beta\right)+e_{i}, \quad i=1, \ldots, n
$$

Esta relação pode ser linear ou não, dependendo da expressão que se considera em $f_{i}\left(X_{i} ; \beta\right)$. Podemos considerar $Y_{i}$ como a variável dependente, enquanto $X_{i}$ pode ser definida como a variável ou conjunto de variáveis independentes ou regressoras, sendo estas em muitos casos conhecidas, $e_{i}$ pode ser definida como o erro do modelo (1.1). Muitas vezes a variável ou conjunto de variáveis $\mathrm{X}$ pode ser não observada ou desconhecida na pesquisa, conforme apresenta Fuller (1987) em uma situação onde se tinha interesse em avaliar o rendimento da produção de milho em função da verdadeira porcentagem de nitrogênio no solo. Aqui considerando a produção do milho como a variável dependente (ou $Y_{i}$ ), enquanto a verdadeira porcentagem de nitrogênio no solo como a variável independente (ou $X_{i}$ ), um possível modelo seria

$$
Y_{i}=\beta_{0}+\beta_{1} X_{i}+e_{i}
$$


mas a verdadeira porcentagem de nitrogênio no solo não pode ser conhecida, o que nos leva a observar seu valor segundo a relação seguinte:

$$
W_{i}=X_{i}+u_{i}
$$

onde $W_{i}$ é o valor que substitui a verdadeiro porcentagem de nitrogênio no solo e $u_{i}$ é considerado como o erro dessa substituição.

As expressões (1.2) e (1.3) constituem as bases dos modelos de regressão com erros nas variáveis, se distinguem dois tipos de modelos em função da expressão (1.3). Se $X_{i}$ é fixo ou constante de modo que o modelo é chamado modelo funcional, podendo ser uma das suposições

$$
\left(\begin{array}{c}
e_{i} \\
u_{i}
\end{array}\right) \sim N_{2}\left[\left(\begin{array}{l}
0 \\
0
\end{array}\right),\left(\begin{array}{cc}
\sigma_{e}^{2} & 0 \\
0 & \sigma_{u}^{2}
\end{array}\right)\right] \text {, independentes, } i=1, \ldots, n
$$

onde $N_{p}(\mu ; \Sigma)$ denota a distribuição normal p-variada com vetor de médias $\mu$ e matriz de covariâncias $\Sigma$. Neste caso o número de parâmetros cresce com o número de observações, tendo-se nas expressões (1.2) e (1.3) um total de $n+4$ parâmetros e neste caso $X_{1}, X_{2}, \ldots, X_{n}$ são denominados parâmetros incidentais. No caso em que na expressão (1.3) $X_{i}$ é uma variável aleatória, então o modelo é chamado modelo estrutural, sendo comum supormos que,

$$
\left(\begin{array}{c}
X_{i} \\
e_{i} \\
u_{i}
\end{array}\right) \sim N_{3}\left[\left(\begin{array}{c}
\mu_{X} \\
0 \\
0
\end{array}\right),\left(\begin{array}{ccc}
\sigma_{X}^{2} & 0 & 0 \\
0 & \sigma_{e}^{2} & 0 \\
0 & 0 & \sigma_{u}^{2}
\end{array}\right)\right] \text {, independentes, } i=1, \ldots, n \text {. }
$$

Neste caso o modelo não apresenta parâmetros incidentais, apenas parâmetros estruturais. Para estes tipos de modelo existe uma variedade de formas para a estimação dos parâmetros $\beta$ e dos demais parâmetros. Neste trabalho apresentamos algumas formas de estimação dos parâmetros encontrados na literatura quando a função $f_{i}\left(X_{i}\right)$ é considerada como um polinômio em $X_{i}, i=1, \ldots, n$, considerando os modelos funcional e estrutural. 


\subsection{Alguns trabalhos em modelos polinomiais com erros nas variáveis.}

Desde começos dos anos 80 , tem havido um interesse crescente no estudo de modelos não lineares. A obtenção de estimadores consistentes dos parâmetros desconhecidos em modelos com erros nas variáveis não lineares resulta muito complicada, mesmo quando o erro de medição é assumido normalmente distribuído, com variância conhecida. A dificuldade é devida ao fato de que as verdadeiras covariáveis desconhecidas devem ser removidas da função de verossimilhança, seja por integração no caso de um modelo estrutural, seja incluindo-as no espaço paramétrico, no caso de um modelo funcional. Os cálculos podem ser bastante complicados. Existem uma variedade de técnicas que produzem estimadores "aproximadamente consistentes" em modelos não lineares, fazendo correções explícitas para o vicio dos estimadores ou propondo estimadores alternativos sob certas suposições. A continuação apresentaremos brevemente algumas soluções propostas para os modelos polinomiais.

Wolter e Fuller (1982) estudaram o polinômio de segundo grau, para isto, consideram o modelo

$$
\begin{gathered}
Y_{i}=\beta_{0}+\beta_{1} X_{i}+\beta_{2} X_{i}^{2}+e_{i} \\
W_{i}=X_{i}+u_{i},
\end{gathered}
$$

$i=1, \ldots, n$. O modelo acima considera que $X_{i}$ não é observado diretamente, sendo que $W_{i}$ é observado no lugar de $X_{i}$. Eles consideram a matriz de covariâncias dos erros,

$$
V=\left(\begin{array}{cc}
\sigma_{e}^{2} & \sigma_{e u} \\
\sigma_{u e} & \sigma_{u}^{2}
\end{array}\right),
$$

conhecida, de modo que da equação (1.5) podemos escrever

$$
\varpi_{\mathbf{i}}=\left(\begin{array}{c}
1 \\
W_{i} \\
W_{i}^{2}-\sigma_{u}^{2}
\end{array}\right)=\left(\begin{array}{c}
1 \\
X_{i} \\
X_{i}^{2}
\end{array}\right)+\left(\begin{array}{c}
0 \\
u_{i} \\
2 X_{i} u_{i}+u_{i}^{2}-\sigma_{u}^{2}
\end{array}\right)=\mathbf{x}_{\mathbf{i}}+\mathbf{f}_{\mathbf{i}}
$$

e, de (1.4) e (1.6), podemos escrever: 


$$
\begin{gathered}
\mathrm{Z}_{i}=\left(\begin{array}{c}
Y_{i} \\
\varpi_{\mathbf{i}}
\end{array}\right)=\left(\begin{array}{c}
\beta_{0}+\beta_{1} X_{i}+\beta_{2} X_{i}^{2} \\
1 \\
X_{i} \\
X_{i}^{2}
\end{array}\right)+\left(\begin{array}{c}
e_{i} \\
0 \\
u_{i} \\
2 X_{i} u_{i}+u_{i}^{2}-\sigma_{u}^{2}
\end{array}\right) \quad \mathrm{ou} \\
\mathbf{Z}_{i}=\mathbf{z}_{i}+\varepsilon_{i}, \quad i=1, \ldots, n .
\end{gathered}
$$

Com isso pode-se construir a matriz de momentos

$$
\mathrm{M}=\frac{1}{n} \sum_{i=1}^{n} \mathrm{Z}_{\mathrm{i}} \mathrm{Z}_{\mathrm{i}}^{\mathrm{t}}=\left(\begin{array}{ll}
\mathrm{M}_{\mathrm{YY}} & \mathrm{M}_{\mathrm{Y} \varpi} \\
\mathrm{M}_{\varpi \mathbf{Y}} & \mathrm{M}_{\varpi \varpi}
\end{array}\right)
$$

sendo

$$
\mathbf{M}_{\mathbf{Y} Y}=\frac{1}{n} \sum_{i=1}^{n} Y_{i}^{2}, \quad \mathbf{M}_{\mathbf{Y} \varpi}=\left(\frac{1}{n} \sum_{i=1}^{n} Y_{i} \quad, \frac{1}{n} \sum_{i=1}^{n} Y_{i} W_{i} \quad, \quad \frac{1}{n} \sum_{i=1}^{n} Y_{i}\left(W_{i}^{2}-\sigma_{u}^{2}\right)\right)
$$

e

$$
\mathbf{M}_{\varpi \varpi}=\left(\begin{array}{ccc}
1 & \frac{1}{n} \sum_{i=1}^{n} W_{i} & \frac{1}{n} \sum_{i=1}^{n}\left(W_{i}^{2}-\sigma_{u}^{2}\right) \\
\frac{1}{n} \sum_{i=1}^{n} W_{i} & \frac{1}{n} \sum_{i=1}^{n} W_{i}^{2} & \frac{1}{n} \sum_{i=1}^{n}\left(W_{i}^{3}-\sigma_{u}^{2} W_{i}\right) \\
\frac{1}{n} \sum_{i=1}^{n}\left(W_{i}^{2}-\sigma_{u}^{2}\right) & \frac{1}{n} \sum_{i=1}^{n}\left(W_{i}^{3}-\sigma_{u}^{2} W_{i}\right) & \frac{1}{n} \sum_{i=1}^{n}\left(W_{i}^{4}-2 \sigma_{u}^{2} W_{i}^{2}+\sigma_{u}^{4}\right)
\end{array}\right),
$$

a matriz de covariâncias $\Omega$ é definida pela expressão,

$$
\Omega=\frac{1}{n} \sum_{i=1}^{n} E\left(\varepsilon_{\mathbf{i}} \varepsilon_{\mathbf{i}}^{\mathbf{t}}\right)=\left(\begin{array}{cc}
\sigma_{e}^{2} & \Omega_{\mathrm{ef}} \\
\Omega_{\mathrm{fe}} & \Omega_{\mathrm{ff}}
\end{array}\right),
$$

sendo que,

$\Omega_{\mathrm{ef}}=\left(\begin{array}{ccc}0 & \sigma_{e u} & 2 \sigma_{e u}^{2} \frac{1}{n} \sum_{i=1}^{n} X_{i}\end{array}\right)$ e $\Omega_{\mathrm{ff}}=\left(\begin{array}{ccc}0 & 0 & 0 \\ 0 & \sigma_{u}^{2} & 2 \sigma_{u}^{2} \frac{1}{n} \sum_{i=1}^{n} X_{i} \\ 0 & 2 \sigma_{u}^{2} \frac{1}{n} \sum_{i=1}^{n} X_{i} & 2 \sigma_{u}^{2}\left(2 \frac{1}{n} \sum_{i=1}^{n} X_{i}^{2}+\sigma_{u}^{2}\right)\end{array}\right)$.

Um estimador não viciado de $\Omega$, isto é, $\hat{\Omega}$ é construído ao substituir $X_{i}$ por $W_{i}$ e $X_{i}^{2}$ por $W_{i}^{2}-\sigma_{u}^{2}$, de forma que o estimador dos $\beta$ é definido pela expressão seguinte, 


$$
\hat{\beta}=\left(M_{\varpi \varpi}-\hat{\alpha} \hat{\Omega}_{\mathrm{ff}}\right)^{-1}\left(\mathrm{M}_{\varpi \mathrm{Y}}-\hat{\alpha} \hat{\Omega}_{\mathrm{fe}}\right)
$$

onde o valor de $\hat{\alpha}$ é obtido resolvendo a equação determinantal $|\mathrm{M}-\alpha \Omega|=0$, os autores afirmam que existem duas motivações para o estimador proposto na expressão (1.7):

1. Pode-se mostrar que $\hat{\alpha}$ converge em probabilidade a 1 , e por sua vez pode-se observar que $\mathbf{M}_{\varpi \varpi}-\hat{\alpha} \hat{\Omega}_{\mathrm{ff}} \quad$ é $\quad$ um estimador $\quad$ de $\mathbf{M}_{\mathbf{X X}} \quad$ e $\mathbf{M}_{\varpi \mathbf{Y}}-\hat{\alpha} \hat{\Omega}_{\mathrm{fe}}$ é um estimador de $\mathrm{M}_{\mathrm{XY}}$.

2. O estimador apresentado na expressão (1.7) é tal que minimiza a função $h(\theta)=$

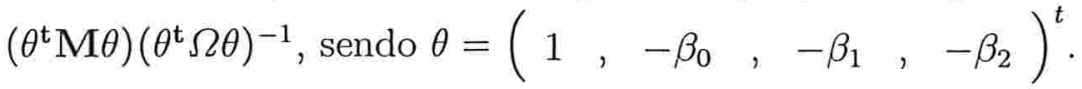

Segundo os autores, em modelos lineares com erros nas variáveis normais $h(\theta)$ pode ser considerada como função de verossimilhança.

Hwang(1986) considera o modelo seguinte:

$$
\begin{gathered}
Y_{i}=\beta_{0}+\beta_{1} X_{i}+\beta_{2} X_{i}^{2}+\ldots+\beta_{p} X_{i}^{p}+e_{i}, \\
W_{i}=X_{i} u_{i}, \quad i=1, \ldots, n .
\end{gathered}
$$

Neste caso estamos frente a um modelo multiplicativo, as suposições sobre a matriz de covariâncias dos erros quando $X_{i}$ é fixo (modelo funcional) é definido por

$$
\left(\begin{array}{l}
e_{i} \\
u_{i}
\end{array}\right) N_{2}\left[\left(\begin{array}{l}
0 \\
1
\end{array}\right),\left(\begin{array}{cc}
\sigma_{e}^{2} & 0 \\
0 & \sigma_{u}^{2}
\end{array}\right)\right] \text {, independentes, } i=1, \ldots, n,
$$

no caso que $X_{i}$ seja uma variável aleatória (modelo estrutural), isto é, $X_{i} \sim N\left(\mu_{X}, \sigma_{X}^{2}\right)$ independentes as suposições apresentadas acima.

Com a finalidade de converter o modelo multiplicativo (1.9) apresentado acima a um modelo aditivo, o autor considera a seguinte modificação

$$
\log \left(W_{i}\right)=\log \left(X_{i}\right)+\log \left(u_{i}\right)
$$

O autor afirma que esta transformação quebra a linearidade do modelo e pode complicá-lo levando a um modelo não linear. Para evitar este problema, ele trabalha o modelo segundo a equação abaixo, 


$$
Y_{i}=\beta_{0}+\beta_{1} W_{i}+\beta_{2} W_{i}^{2}+\ldots+\beta_{p} W_{i}^{p}+e_{i}
$$

obtendo a partir da expressão acima o estimador ingênuo "naive" da forma

$$
\hat{\beta}_{M Q}=\left(\mathrm{W}^{t} \mathrm{~W}\right)^{-1}\left(\mathrm{~W}^{t} \mathrm{Y}\right)
$$

que não é consistente. O autor prova pela lei dos grandes que se pode obter a seguinte expressão

$$
\frac{\mathbf{W}^{t} \mathbf{Y}}{n} \stackrel{P}{\longrightarrow} \mathrm{A} \beta
$$

onde $\stackrel{P}{\longrightarrow}$ denota convergência em probabilidade, quando $n \rightarrow \infty$. Sendo

$$
\mathbf{A}=E\left(\mathbf{X}_{1} \mathbf{X}_{1}^{t}\right)
$$

e como A é uma matriz não singular, então

$$
\mathrm{A}^{-1}\left(\frac{\mathrm{W}^{t} \mathrm{Y}}{n}\right) \stackrel{P}{\longrightarrow} \beta .
$$

Para a determinação de $\mathbf{A}$, notemos que, sob condições de regularidade,

$$
\frac{\mathrm{W}^{t} \mathrm{~W}}{n} \stackrel{P}{\longrightarrow} E\left(\mathrm{~W}_{1} \mathrm{~W}_{1}^{t}\right)
$$

e levando em conta que $E\left(\mathbf{W}_{1} \mathbf{W}_{1}^{t}\right)=\mathbf{M} \odot E\left(\mathrm{X}_{1} \mathbf{X}_{1}^{t}\right)$, onde $\mathbf{M}=E\left(\mathbf{u}_{1} \mathbf{u}_{1}^{t}\right)$, para esta expressão supomos que $u_{i} \sim N\left(1, \sigma_{u}^{2}\right)$, e $\sigma_{u}^{2}$ é conhecida, porém a matriz $\mathbf{M}$, também é conhecida, com isto temos que,

$$
E\left(\mathrm{~W}_{1} \mathrm{~W}_{1}^{t}\right) \oslash \mathrm{M}=E\left(\mathrm{X}_{1} \mathrm{X}_{1}^{t}\right)=\mathbf{A} .
$$

O autor conclui segundo a expressão (1.11), que

$$
\hat{\beta}=\left[\left(\mathrm{W}^{t} \mathrm{~W}\right) \oslash \mathrm{M}\right]^{-1}\left(\mathrm{~W}^{t} \mathrm{Y}\right)
$$

é um estimador consistente para $\beta$. As operações " $\odot$ "e " $\oslash$ "são os operadores matemáticos do produto e divisão de Hadamard(Styan, 1973), que são o produto e a divisão elemento a elemento de duas matrizes com a mesma dimensão.

Hwang (1986) apresenta, usando o teorema central do limite multivariada (Sen e Singer, 1992), a distribuição assintótica dos estimadores, ou seja, 


$$
\sqrt{n}(\hat{\beta}-\beta) \stackrel{D}{\longrightarrow} N_{p}(0 ; \Sigma), \quad \text { quando } n \rightarrow \infty
$$

onde, $\Sigma$ é estimada por

$$
\hat{\Sigma}=\hat{\mathrm{A}}^{-1} \sum_{i=1}^{n}\left(\mathrm{~W}_{i} Y_{i}-\left(\mathrm{W}_{i} \mathrm{~W}_{i}^{t} \odot \mathrm{M}\right) \hat{\beta}\right)\left(\mathrm{W}_{i} Y_{i}-\left(\mathrm{W}_{i} \mathrm{~W}_{i}^{t} \odot \mathbf{M}\right) \hat{\beta}\right)^{t} \hat{\mathrm{A}}^{-1}
$$

com $\mathrm{M}$ conhecida.

Carroll, Rupert e Stefanski (1995), obtiveram estimadores consistentes a partir da expressão (1.10) que é o modelo ingênuo ( "naive"), considerando o algoritmo SIMEX (Extrapolação da Simulação) desenvolvido por Cook e Stefanski (1995). O procedimento envolve duas etapas, sendo a primeira etapa chamada de etapa de simulação, onde se consideram modificações nas variáveis observáveis do seguinte modo:

$$
W_{i, s}(\lambda)=W_{i}+\sqrt{\lambda} u_{i}
$$

$i=1,2, \ldots, n$, e $s=1,2, \ldots, B$, onde $u_{i} \sim N\left(0, \sigma_{u}^{2}\right)$. Para cada valor de $\lambda$ fixado (valor compreendido entre 0 e 2 ), deve-se simular $n$ possíveis valores para $W_{i, s}(\lambda)$; a seguir deve-se usar a técnica de mínimos quadrados para a obtenção dos $\beta$ 's para cada $\lambda$ fixo; este processo deve-se repetir $\mathrm{B}$ vezes, de modo que para cada valor fixo de $\lambda$ se obtém um valor médio de $\hat{\beta}$, da maneira seguinte,

$$
\hat{\beta}\left(\lambda_{j}\right)=\frac{1}{B} \sum_{s=1}^{B} \hat{\beta}_{s}\left(\lambda_{j}\right), j=1, \ldots, p .
$$

Na segunda etapa, chamada de extrapolação, deve-se estimar uma regressão entre cada valor de $\hat{\beta}\left(\lambda_{j}\right)$ e os valores de $\lambda_{j}$ fixos, sendo que algumas relações sugeridas na literatura são:

$$
\begin{array}{lr}
\hat{\beta}\left(\lambda_{j}\right)=\gamma_{0}+\gamma_{1} \lambda_{j}, & \text { (Extrapolação Linear) } \\
\hat{\beta}\left(\lambda_{j}\right)=\gamma_{0}+\gamma_{1} \lambda_{j}+\gamma_{2} \lambda_{j}^{2}, & \text { (Extrapolação Quadrática) } \\
\hat{\beta}\left(\lambda_{j}\right)=\gamma_{0}+\frac{\gamma_{1}}{\gamma_{2}+\lambda_{j}} . & \text { (Extrapolação Não Linear) }
\end{array}
$$

Uma vez estimados os parâmetros $\gamma_{m}$, para $m=0,1,2$ deve-se fazer $\lambda=-1$, obtendo-se um estimativa de cada parâmetro $\beta$,

$$
\hat{\beta}_{\text {SIMEX }}=\hat{\beta}_{(\lambda=-1)} .
$$


O método SIMEX foi feito para se trabalhar com o método estrutural, mas também pode se trabalhar com o modelo funcional.

Para um modelo multiplicativo como na expressão (1.9) quando é usado o SIMEX pode-se substituir na etapa de simulação, a equação (1.12), pela expressão seguinte:

$$
W_{i, s}(\lambda)=e^{\log \left(W_{i}\right)+\sqrt{\lambda} u_{i}}
$$

mantendo-se os outros procedimentos para estimar os parâmetros de $\beta$ sem nenhuma modificação.

Cheng e Schneeweis (1998), trabalharam o modelo como as equações (1.8) e (1.5), considerando a técnica de mínimos quadrados ordinários, obtêm as seguintes equações lineares:

$$
\left(\mathrm{X}^{t} \mathrm{X}\right) \hat{\beta}_{M Q}=\mathrm{X}^{t} \mathrm{Y}
$$

substituindo a matriz $\left(\mathrm{X}^{t} \mathrm{X}\right)$ que não é observável, de acordo com o modelo (1.5) por uma outra matriz $\mathbf{H}$, constituída por variáveis auxiliares $t_{i,(r)}$ que representam os momentos da variável não observada, $X_{i}^{r}$, isto é, $E\left(t_{i,(r)}\right)=X_{i}^{r}$, esta variável auxiliar está em função de $W_{i}^{r}$ e $\sigma_{u}^{2}$, relacionadas através da equação (com $\sigma_{u}^{2}$ conhecido)

$$
t_{i,(r+1)}=W_{i} t_{i,(r)}-r \sigma_{u}^{2} t_{i,(r-1)} \quad r>2,
$$

da mesma forma, o vetor $\left(\mathrm{X}^{\mathrm{t}} \mathrm{Y}\right)$ é substituído pelo vetor $\mathrm{h}$, constituído pelas variáveis $t_{i,(r)}$, de tal forma que um estimador consistente de $\beta$ pode ser obtido a partir da equação,

$$
\mathbf{H} \hat{\beta}=\mathbf{h},
$$

onde um estimador de $\sigma_{e}^{2}$ é dado por

$$
\hat{\sigma}_{e}^{2}=\frac{1}{n}\left(\mathrm{Y}^{t} \mathrm{Y}-\mathrm{h}^{t} \mathrm{H}^{-1} \mathrm{~h}\right) .
$$

Os autores denominam esta forma de se obter as estimativas como estimador de mínimos quadrados ajustados. Usando propriedades assintóticas de equações de estimação (Huber,1981), eles apresentam o comportamento assintótico de $\hat{\beta}$, como sendo 


$$
\sqrt{n}(\hat{\beta}-\beta) \stackrel{D}{\longrightarrow} N_{p}(0 ; \Sigma)
$$

onde

$$
\hat{\Sigma}=\frac{1}{n}\left(\overline{\mathrm{H}}^{-1} \hat{\mathrm{V}} \overline{\mathrm{H}}^{-1}\right) \quad \text { e } \quad \hat{\mathrm{V}}=\frac{1}{n} \sum_{i=1}^{n}\left(\mathrm{H}_{i} \hat{\beta}-\mathrm{h}_{i}\right)\left(\mathrm{H}_{i} \hat{\beta}-\mathrm{h}_{i}\right)^{t} .
$$

Esta técnica é apropriada para modelos funcionais, assim como para modelos estruturais. Por outro lado o método exige o conhecimento dos momentos da variável $u_{i}, i=1, \ldots, n$.

Conforme visto na Introdução, podemos advertir que nos modelo com erros nas variáveis existem duas formas de enfocar à variável $X_{i}$ que é não observável; uma primeira forma trata $X_{i}$ como uma variável aleatória (modelo estrutural), uma segunda forma trata $X_{i}$ como uma constante fixa (modelo funcional), mas na realidade existe uma terceira situação que é similar ao modelo funcional, a forma como é apresentada faz a diferença. Suponhamos que desejemos conduzir um experimento para estudar a qualidade de determinada marca de cimento e para isto, é criado uma operação de mistura constante. Assuma que a qualidade do cimento depende da quantidade de água usada na mistura. A leitura da água pode ser feita através do medidor da válvula de controle da quantidade de água que ingressa na mistura. A quantidade de água que se requer na mistura é constante e conhecida $\left(W_{i}\right)$, mas devido à pressão da água, pode-se enviar mais quantidade de água do que se está observando no medidor, podendo-se entender que esse excesso corresponde ao erro pela medição da água $\left(u_{i}\right), i=1, \ldots, n$.

Se o medidor está apropriadamente calibrado, a média de $u_{i}$ é zero. Temos então a relação

$$
X_{i}=W_{i}-u_{i}
$$

onde $u_{i}$ é uma variável aleatória de média zero e variância $\sigma_{u}^{2}$. Como se deseja uma qualidade ótima do cimento, a quantidade de água através do medidor da válvula de controle de água $W_{i}$ deve ser pré-fixado pelo pesquisador e as possíveis variações são devidas ao erro aleatório $u_{i}$. Este modelo é chamada modelo de Berkson (Fuller, 1987). Observemos que a variável $W_{i}$ pode ser chamada variável controlada, pois seus valores são fixados com antecedência, 
enquanto a variável desconhecida $X_{i}$ é flutuante em seus valores.

Cheng e Van Ness (1998), estudaram o modelo polinomial de Berkson considerando as equações

$$
\begin{gathered}
Y_{i}=\beta_{0}+\beta_{1} X_{i}+\beta_{2} X_{i}^{2}+\ldots+\beta_{p} X_{i}^{p}+e_{i}=P\left(\mathrm{X}_{i} ; \beta\right)+e_{i}, \\
W_{i}=X_{i}+u_{i},
\end{gathered}
$$

logo a expressão (1.15) pode ser escrita por $X_{i}=W_{i}-u_{i}$ e substituída na expressão (1.14), obtendo-se

$$
Y_{i}=\beta_{0}+\beta_{1} W_{i}+\beta_{2} W_{i}^{2}+\ldots+\beta_{p} W_{i}^{p}+e_{i}^{*}=P\left(\mathrm{~W}_{i} ; \beta\right)+e_{i}^{*},
$$

com $e_{i}^{*}=P\left(\mathrm{X}_{i} ; \beta\right)-P\left(\mathbf{W}_{i} ; \beta\right)+e_{i}$, de modo que o valor esperado de $e_{i}^{*}$ não é zero, somando e subtraindo $E\left(e_{i}^{*}\right)$ em (1.16), obtém-se

$$
Y_{i}=\beta_{0}+E\left(e_{i}^{*}\right)+\beta_{1} W_{i}+\beta_{2} W_{i}^{2}+\ldots+\beta_{p} W_{i}^{p}+e_{i}^{*}-E\left(e_{i}^{*}\right),
$$

$\operatorname{com} e_{i}^{* *}=e_{i}^{*}-E\left(e_{i}^{*}\right)$ e $E\left(e_{i}^{* *}\right)=0$, lembrando que os $E\left(e_{i}^{*}\right)$ estão em função dos $\beta$ 's e $W_{i}$, obtemos o seguinte resultado

$$
Y_{i}=\beta_{0}^{*}+\beta_{1} W_{i}+\beta_{2} W_{i}^{2}+\ldots+\beta_{p} W_{i}^{p}+e_{i}^{* *}
$$

sendo que em (1.17) os parâmetros $\beta$ podem ser estimados com a técnica de mínimos quadrados ordinários e neste caso os estimadores são consistentes e eficientes. Variâncias dos estimadores também são estimados usando procedimentos usuais.

\subsection{O método para a correção de viés em modelos com erros nas variáveis.}

O método da função verossimilhança corrigida foi desenvolvido por Nakamura (1990) e o método da função escore corrigida foi desenvolvido por Bolfarine e Gimenez (1997), que apresentam a descrição do método, as propriedades assintóticas dos estimadores, testes de hipóteses e também aplicações ao modelo de calibração comparativa.

As técnicas da função de verossimilhança e do escore corrigido podem ser usadas tanto 
para estruturas aditivas dos erros quanto para multiplicativas, assim como para modelos funcionais e para modelos estruturais.

A propriedade fundamental que define uma função de verossimilhança corrigida é o fato de que sua esperança com respeito à distribuição dos erros de medição e as esperanças das derivadas parciais com respeito a cada parâmetro (função escore corrigido a partir da verossimilhança) coincidem com a função de escore usual baseada nas verdadeiras covariáveis que são desconhecidas.

Esta propriedade é importante para provar a consistência e a normalidade assintótica do estimador obtido como solução da equação de estimação obtida com o escore corrigido.

Em resumo, se temos o logaritmo da função de verossimilhança não observada,

$$
\ell(\theta ; \mathrm{X}, \mathrm{Y})=\sum_{i=1}^{n} \ell_{i}\left(\theta, X_{i}, Y_{i}\right)
$$

e uma função de escore não observada,

$$
\mathrm{U}(\theta ; \mathrm{X}, \mathrm{Y})=\frac{\partial \ell}{\partial \theta}(\theta ; \mathrm{X}, \mathrm{Y})
$$

temos que

$$
E[\mathrm{U}(\theta ; \mathrm{X}, \mathrm{Y})]=0
$$

sendo esta propriedade fundamental para consistência e normalidade do estimador que hboxmaximiza $\ell(\theta ; \mathrm{X}, \mathrm{Y})$.

Se substituímos a variável não observável $X_{i}$ pela variável observável $W_{i}$ temos que,

$$
E[\mathbf{U}(\theta ; \mathrm{W}, \mathrm{Y})] \neq 0 \text {. }
$$

Deve-se procurar modificações de (1.19) tal que se obtenha a propriedade (1.18), daí a necessidade de se obter $\mathbf{U}^{*}(\theta ; \mathrm{W}, \mathrm{Y})$ satisfazendo

$$
E\left[\mathbf{U}^{*}(\theta ; \mathbf{W}, \mathbf{Y})\right]=0
$$

e a partir desta expressão podemos obter estimadores consistentes. 
Segundo Bolfarine e Gimenez (1997), um estimador consistente de $\theta$ poderia ser obtido como solução de uma equação de estimação não viciada. Nakamura (1990) propõe obter tal função da seguinte maneira. Seja $\ell^{*}(\theta ; \mathrm{X}, \mathrm{Y})$ uma função das variáveis observadas e do parâmetro desconhecido tal que

$$
E\left[\ell^{*}(\theta ; \mathrm{W}, \mathrm{Y}) \mid \mathrm{X}, \mathrm{Y}\right]=\ell(\theta ; \mathrm{X}, \mathrm{Y})
$$

$\ell^{*}(\theta ; \mathrm{W}, \mathrm{Y})$ é chamada de logaritmo da função verossimilhança corrigida.

Se $\ell^{*}(\theta ; \mathbf{W}, \mathbf{Y})$ for duas vezes diferenciável em $\digamma$, onde $\digamma$ é um sub-conjunto aberto e conexo do espaço paramétrico $\Theta$, definem-se

$$
\mathbf{U}^{*}(\theta ; \mathbf{W}, \mathbf{Y})=\frac{\partial}{\partial \theta} \ell^{*}(\theta ; \mathbf{W}, \mathbf{Y})
$$

e

$$
\mathbf{I}^{*}(\theta ; \mathbf{W}, \mathbf{Y})=\frac{\partial}{\partial \theta} \mathbf{U}^{*}(\theta ; \mathbf{W}, \mathbf{Y})
$$

a função escore corrigido e a matriz de informação observada corrigida, respectivamente.

Uma condição importante no método de Nakamura, é que as operações $E(\bullet \mid \mathrm{X}, \mathrm{Y})$ e $\frac{\partial}{\partial \theta}(\bullet)$ devem ser permutáveis, o que permite provar a seguinte condição:

$$
E\left[\mathbf{U}^{*}(\theta ; \mathbf{W}, \mathbf{Y}) \mid \mathbf{X}, \mathbf{Y}\right]=\mathbf{U}(\theta ; \mathrm{X}, \mathrm{Y}), \quad \forall \theta \in \digamma
$$

de modo que (1.20) estaria satisfeita.

O valor de $\theta$ obtida a partir de $\mathrm{U}^{*}(\hat{\theta} ; \mathrm{W}, \mathrm{Y})=0$ é dito estimador do escore corrigido de $\theta$.

Bolfarine e Gimenez, (1997) apresentam as condições de regularidade que devem satisfazer a função escore corrigido, bem como um estudo da consistência e da normalidade assintótica dos estimadores, sendo a matriz de covariâncias assintótica dos estimadores dada por

$$
\frac{1}{n}\left(\mathbf{I}_{n}^{*}(\hat{\theta} ; \mathrm{W}, \mathrm{Y})\right)^{-1} \mathbf{S}_{n}^{*}(\hat{\theta} ; \mathrm{W}, \mathrm{Y})\left(\mathbf{I}_{n}^{*}(\hat{\theta} ; \mathbf{W}, \mathbf{Y})\right)^{-1}
$$


onde,

$$
\mathbf{I}_{n}^{*}(\hat{\theta} ; \mathrm{W}, \mathrm{Y})=-\frac{1}{n} \sum_{i=1}^{n} \frac{\partial}{\partial \theta} U_{i}^{*}(\hat{\theta} ; \mathrm{W}, \mathrm{Y})
$$

e

$$
\mathrm{S}_{n}^{*}(\hat{\theta} ; \mathrm{W}, \mathrm{Y})=\frac{1}{n} \sum_{i=1}^{n} \mathrm{U}_{i}^{*}(\hat{\theta} ; \mathrm{W}, \mathrm{Y}) \mathrm{U}_{i}^{*}(\hat{\theta} ; \mathrm{W}, \mathrm{Y})^{t}
$$

Segundo os autores, o método do escore corrigido é aplicável à distribuições de poisson, normal, normal inversa, valor extremo e gama. Em geral o escore corrigido pode ser determinado quando o escore usual (não observado) depende das covariáveis somente através de potências e produtos dos mesmos. Os autores fazem um estudo dos modelos de calibração comparativa usando o método de escore corrigido, onde se define como modelo de calibração comparativa o modelo que permite comparar instrumentos ou métodos de medição (capítulo 4). Estes modelos são casos particulares dos modelos com erros nas variáveis multivariados, segundo Fuller, (1987).

\subsection{Influência Local}

Modelos estatísticos são ferramentas úteis para extrair e compreender as características essenciais de um conjunto de dados. Modelos são quase sempre, descrições aproximadas de processos reais bem complexos e portanto são quase sempre inexatos. Devido a estas inexatidões, o estudo do efeito de pequenas modificações na formulação do modelo torna-se importante. Se uma modificação pequena de uma descrição aproximada afeta fortemente os resultados de uma análise, este deve ser o centro de nosso interesse.

Por outro lado, se as alterações encontradas são desprezíveis, o modelo é robusto com relação às perturbações induzidas e nossa ignorância do modelo poderia não causar dano.

Uma técnica geral para avaliar a influência de pequenas perturbações no modelo ou nos dados é proposta por Cook (1986), que se baseia na comparação dos modelos postulado e perturbado fazendo $v$ variar em $\Omega$, procurando elementos em $v$ responsáveis pelo afastamento entre os modelos. Este método é conhecido como método de influência local. Permite avaliar a influência que pequenas perturbações podem exercer sobre componentes do modelo, tais como estimativa dos parâmetros e outros resultados da análise. 
Um estudo sobre influência local no método de escore corrigido foi trabalhada por Lee e Zhao (1996), onde a influência de pequenas perturbações (denotada por $v$ ) podem ser obtidas pelo distanciamento do logaritmo da função verossimilhança corrigida $\ell^{*}(\beta)$,

$$
F(v)=2\left[\ell^{*}(\hat{\beta})-\ell^{*}(\hat{\beta}(v))\right]
$$

notemos que $\ell^{*}(\beta(v))$ corresponde a função verossimilhança corrigida e perturbada.

Cook (1986) aplica conceitos de geometria diferencial para quantificar $F(v)$. Para obter a curvatura normal na direção unitária $d, F(v)$ deve ser uma função não negativa com um mínimo global em $v_{0}$, de tal forma que a curvatura normal em $F\left(v_{0}\right)$ na direção unitária $d$ pode ser expressa como

$$
C(d)=2\left|d^{t} \Delta^{t} f^{-1} \Delta d\right|
$$

onde

$$
\begin{gathered}
\Delta=\frac{\partial^{2} \ell^{*}(\beta \mid v)}{\partial \beta \partial v^{t}}, \\
f=-\frac{\partial^{2} \ell^{*}(\beta)}{\partial \beta \partial \beta^{t}},
\end{gathered}
$$

ambas avaliadas em $v_{0}$ e $\hat{\beta}$.

O diagnóstico da influência é derivado do análise dos autovalores de $\Delta^{t} f \Delta$, isto é, se consideramos $C_{\max }=\max _{d:\|d\|=1} C(d)$ que corresponde ao maior autovalor de $-2 \Delta^{t} f \Delta$ (veja, Seber, 1984; pag.526) e $d_{\text {max }}$ é o autovetor associado ao maior autovalor $C_{\text {max }}$. Neste caso podemos considerar $d_{\max }$ como a direção que produz maior mudança local nas estimativas dos parâmetros, sendo que os elementos mais influentes podem ser identificados através da magnitude relativo dos elementos em $d_{\max }$, enquanto que a sensibilidade em relação a perturbação causada pode ser indicada pelo valor de $C_{\max }$.

Os objetivos do presente trabalho é estudar o método da maxima verossimilhança corrigida proposto por Nakamura (1990) e o método da função escore corrigida desenvolvido por Bolfarine e Gimenez (1997), assim como a influência local que se gera ao elaborar modelos polinomiais gerais com erros nas variáveis, fazendo estudos nos casos de 
erros aditivos e multiplicativos para modelos funcionais e estruturais. Um caso particular de um análise multivariado será fazer um estudo em calibração comparativa.

Para atingir estes objetivos o trabalho foi organizado em quatro capítulos. No Capitulo 2, apresentamos um estudo dos métodos da maxima verossimilhança corrigida e da função escore corrigido quando se trabalha em um modelo polinomial com erros nas variáveis no caso aditivo, considerando modelos funcionais e estruturais. Neste capítulo se faz um estudo da estimação dos parâmetros nos casos quando $\sigma_{u}^{2}$ e $\lambda$ são conhecidas, sendo $\lambda=\sigma_{e}^{2} / \sigma_{u}^{2}$, a obtenção das variâncias assintóticas dos estimadores nos casos em que $\sigma_{u}^{2}$ e $\lambda$ são conhecidas, assim como testes de hipóteses de interesse, um estudo da influência local e um estudo da estimação dos parâmetros quando as variâncias dos erros são heterogêneas, isto é, temos $\sigma_{e_{i}}^{2}$ e $\sigma_{u_{i}}^{2}, i=1, \ldots, n$. Enfatizamos que o caso em que $\lambda=\sigma_{e}^{2} / \sigma_{u}^{2}$ é conhecido não é tratado na literatura por nenhum método. O mesmo se passa com o caso das variâncias heterogêneas.

No Capitulo 3 é abordado o estudo dos mesmos métodos verossimilhança corrigida e a função escore corrigido no modelo polinomial com erros nas variáveis considerando o erros multiplicativos. Tópicos similares aos desenvolvidos no Capitulo 2 também são considerados.

No Capitulo 4, se faz um estudo do método do escore corrigido considerando o caso da calibração comparativa, tanto com variâncias dos erros homogêneas quanto heterogêneas. 


\section{CAPÍTULO}

\subsection{Introdução.}

Nesta seção estudaremos os modelos polinomiais com erros de medição aditivos nas variáveis, que em termos gerais pode ser definido por

$$
\begin{gathered}
Y_{i}=\sum_{j=0}^{p} \beta_{j} X_{i}^{j}+e_{i}, \\
W_{i}=X_{i}+u_{i},
\end{gathered}
$$

$i=1, \ldots, n$. As equações (2.1) e (2.2) constituem um modelo polinomial com erros nas variáveis de tipo aditivo, onde as variáveis $Y_{i}$ e $W_{i}$ são variáveis observáveis, enquanto $X_{i}$ é uma variável não observável, a qual é observada a traves de $W_{i} \cdot \beta_{j}, j=0,1, \ldots, p$ são os parâmetros que se deseja estimar, $e_{i}$ é o erro do modelo proposto e $u_{i}$ é o erro por observar $W_{i}$ e não $X_{i}$, sendo $i=1, \ldots, n$. Se consideramos o modelo funcional o suposto a considerar no presente trabalho é definido pela expressão (1.4), no caso de trabalhar com o modelo estrutural será considerada a expressão (1.5). Devemos destacar que no desenvolvimento do presente trabalho podemos usar qualquer um dos modelos (funcional ou estrutural).

Como foi mencionado no capítulo 1, este modelo foi amplamente estudado sob variadas óticas. Nosso interesse neste capitulo é avaliar os modelos polinomiais acima considerando as metodologias propostas por Nakamura (1990) e Bolfarine e Gimenez (1997). 
A análise se baseia no estudo da função log-verossimilhança corrigida e a partir dela gerar a função escore corrigida. Com a finalidade de verificar a condição de $E(\bullet \mid \mathrm{Y}, \mathrm{X})$ e $\frac{\partial}{\partial \theta}(\bullet)$ serem permutáveis, será avaliada a função escore corrigido sem considerar a função log-verossimilhança corrigida, tendo que coincidir as duas formas de obtenção da função escore corrigido.

Na seção 2.2 estudamos a estimação dos parâmetros, as variâncias assintóticas dos estimadores e os testes de hipóteses para o caso em que $\sigma_{u}^{2}$ é conhecida. Na seção 2.3 apresentamos a estimação dos parâmetros, as variâncias assintóticas e os testes de hipóteses para o caso em que $\lambda=\frac{\sigma_{e}^{2}}{\sigma_{u}^{2}}$ é conhecida. Na seção 2.4 apresentamos um estudo de influência local, considerando alguns esquemas de perturbação e uma aplicação prática do caso 1 será considerada. Na seção 2.5 analisamos o caso em que as variâncias dos erros $\left(\sigma_{e}^{2}\right.$ e $\left.\sigma_{u}^{2}\right)$ são heterogêneas.

\subsection{Caso $1: \sigma_{u}^{2}$ conhecida}

\subsubsection{Estimação dos parâmetros}

Nesta seção estudaremos a função verossimilhança corrigida derivada segundo a proposta de Nakamura (1990), desenvolvimento válido tanto para o modelo funcional quanto para o modelo estrutural.

a) Avaliação da função escore a partir do logaritmo da função verossimilhança "naive"

Para estimar os parâmetros $\beta$ e $\sigma_{\mathbf{e}}^{2}$ consideramos o logaritmo da função verossimilhança "naive", como proposto em Nakamura (1990), dada por

$$
\ell\left(\beta, \sigma_{e}^{2} ; \mathrm{W}, \mathrm{Y}\right)=-\frac{n}{2} \log (2 \pi)-\frac{n}{2} \log \left(\sigma_{e}^{2}\right)-\frac{1}{2 \sigma_{e}^{2}} \sum_{i=1}^{n}\left(Y_{i}-\sum_{j=0}^{p} \beta_{j} W_{i}^{j}\right)^{2},
$$

com a finalidade de se obter,

$$
\begin{aligned}
E\left(\ell^{*}\left(\beta, \sigma_{e}^{2} ; \mathrm{W}, \mathrm{Y}\right)\right) & =E\left[E\left(\ell^{*}\left(\beta, \sigma_{e}^{2} ; \mathrm{W}, \mathrm{Y}\right) \mid \beta, \sigma_{e}^{2} ; \mathrm{X}, \mathrm{Y}\right)\right] \\
& =E\left(\ell\left(\beta, \sigma_{e}^{2} ; \mathrm{X}, \mathrm{Y}\right)\right)
\end{aligned}
$$


da expressão da acima temos que

$$
E\left(\ell^{*}\left(\beta, \sigma_{e}^{2} ; \mathrm{W}, \mathrm{Y}\right) \mid \beta, \sigma_{e}^{2} ; \mathrm{X}, \mathrm{Y}\right)=\ell\left(\beta, \sigma_{e}^{2} ; \mathrm{X}, \mathrm{Y}\right)
$$

Para chegar à expressão (2.4), iniciamos o valor esperado condicional de (2.3). Com a finalidade de simplificar nossa notação, fazemos $K=\left(\beta, \sigma_{e}^{2} ; \mathrm{Y}, \mathrm{X}\right)$. Logo,

$$
\begin{aligned}
E\left(\ell\left(\beta, \sigma_{e}^{2} ; \mathbf{Y}, \mathbf{W}\right) \mid K\right)= & -\frac{n}{2} \log (2 \pi)-\frac{n}{2} \log \left(\sigma_{e}^{2}\right)-\frac{1}{2 \sigma_{e}^{2}} \sum_{i=1}^{n} E\left[\left(Y_{i}-\sum_{j=0}^{p} \beta_{j} W_{i}^{j}\right)^{2} \mid K\right] \\
= & -\frac{n}{2} \log (2 \pi)-\frac{n}{2} \log \left(\sigma_{e}^{2}\right)-\frac{1}{2 \sigma_{e}^{2}}\left\{\sum _ { i = 1 } ^ { n } \left[Y_{i}^{2}+\sum_{j=0}^{p} \beta_{j}^{2} E\left(W_{i}^{2 j} \mid K\right)\right.\right. \\
& \left.\left.-2 Y_{i} \sum_{j=0}^{p} \beta_{j} E\left(W_{i}^{j} \mid K\right)+2 \sum_{j=0}^{p} \sum_{s=0}^{p} \beta_{j} \beta_{s} E\left(W_{i}^{j+s} \mid K\right)\right]\right\} .
\end{aligned}
$$

Considerando na expressão (2.5) o artifício seguinte,

$$
E\left(W_{i}^{m} \mid K\right)=X_{i}^{m}+\left(E\left(W_{i}^{m} \mid K\right)-X_{i}^{m}\right),
$$

temos:

$$
\begin{aligned}
E\left(\ell\left(\beta, \sigma_{e}^{2} ; \mathrm{Y}, \mathrm{W}\right) \mid K\right)= & -\frac{n}{2} \log (2 \pi)-\frac{n}{2} \log \left(\sigma_{e}^{2}\right)-\frac{1}{2 \sigma_{e}^{2}}\left\{\sum _ { i = 1 } ^ { n } \left[Y_{i}^{2}+\sum_{j=0}^{p} \beta_{j}^{2} X_{i}^{2 j}\right.\right. \\
& \left.\left.-2 Y_{i} \sum_{j=0}^{p} \beta_{j} X_{i}^{j}+2 \sum_{j=0}^{p} \sum_{s=0}^{p} \beta_{j} \beta_{s} X_{i}^{j+s}\right]\right\} \\
& +\frac{1}{2 \sigma_{e}^{2}}\left\{\sum _ { i = 1 } ^ { n } \left[\sum_{j=0}^{p} \beta_{j}^{2}\left(E\left(W_{i}^{2 j} \mid K\right)-X_{i}^{2 j}\right)\right.\right. \\
& -2 Y_{i} \sum_{j=0}^{p} \beta_{j}\left(E\left(W_{i}^{j} \mid K\right)-X_{i}^{j}\right) \\
& \left.\left.+2 \sum_{j=0}^{p} \sum_{s=0}^{p} \beta_{j} \beta_{s}\left(E\left(W_{i}^{j+s} \mid K\right)-X_{i}^{j+s}\right)\right]\right\}
\end{aligned}
$$


Na expressão (2.7) podemos identificar duas componentes. Uma primeira que corresponde ao logaritmo da função verossimilhança não observada $\left(\ell\left(\beta, \sigma_{e}^{2} ; \mathrm{Y}, \mathrm{X}\right)\right)$ e uma segunda que corresponde ao vício esperado condicional do logaritmo da função verossimilhança não corrigida, sendo este vício o seguinte:

$$
\begin{aligned}
\text { vício }= & \frac{1}{2 \sigma_{e}^{2}}\left\{\sum _ { i = 1 } ^ { n } \left[\sum_{j=0}^{p} \beta_{j}^{2}\left(E\left(W_{i}^{2 j} \mid K\right)-X_{i}^{2 j}\right)-2 Y_{i} \sum_{j=0}^{p} \beta_{j}\left(E\left(W_{i}^{j} \mid K\right)-X_{i}^{j}\right)+\right.\right. \\
& \left.\left.+2 \sum_{\substack{j=0 \\
s<j}}^{p} \sum_{\substack{s=0 \\
s}}^{p} \beta_{j} \beta_{s}\left(E\left(W_{i}^{j+s} \mid K\right)-X_{i}^{j+s}\right)\right]\right\} .
\end{aligned}
$$

Considerando a substituição de variáveis como em Cheng e Schneeweis (1998), isto é, $E\left(W_{i}^{m} \mid K\right)$ por $W_{i}^{m}$ e $X_{i}^{m}$ por $t_{i,(m)}$ na expressão (2.8), sendo $t_{i,(m)}$ a variável auxiliar observada através de $W_{i}^{m}$, e calculada como na expressão(1.13), temos que

$$
\begin{aligned}
\text { vício* }^{*}= & \frac{1}{2 \sigma_{e}^{2}}\left\{\sum _ { i = 1 } ^ { n } \left[\sum_{j=0}^{p} \beta_{j}^{2}\left(W_{i}^{2 j}-t_{i,(2 j)}\right)-2 Y_{i} \sum_{j=0}^{p} \beta_{j}\left(W_{i}^{j}-t_{i,(j)}\right)\right.\right. \\
& \left.\left.+2 \sum_{\substack{j=0 \\
s<j}}^{p} \sum_{s=0}^{p} \beta_{j} \beta_{s}\left(W_{i}^{j+s}-t_{i,(j+s)}\right)\right]\right\} .
\end{aligned}
$$

Agora o logaritmo da função de verossimilhança corrigida pode ser considerada segundo a seguinte expressão:

$$
\ell^{*}\left(\beta, \sigma_{e}^{2} ; \mathrm{Y}, \mathrm{W}\right)=\ell\left(\beta, \sigma_{e}^{2} ; \mathrm{Y}, \mathrm{W}\right)-\text { vício*}^{*}
$$

Fazendo alguns cálculos algébricos temos que o logaritmo da função verossimilhança corrigida é dado por 


$$
\begin{aligned}
\ell^{*}\left(\beta, \sigma_{e}^{2} ; \mathrm{Y}, \mathrm{W}\right)= & -\frac{n}{2} \log (2 \pi)-\frac{n}{2} \log \left(\sigma_{e}^{2}\right)-\frac{1}{2 \sigma_{e}^{2}}\left\{\sum _ { i = 1 } ^ { n } \left[Y_{i}^{2}+\sum_{j=0}^{p} \beta_{j}^{2} t_{i,(2 j)}\right.\right. \\
& \left.\left.-2 Y_{i} \sum_{j=0}^{p} \beta_{j} t_{i,(j)}+2 \sum_{j=0}^{p} \sum_{s=0}^{p} \beta_{j} \beta_{s} t_{i,(j+s)}\right]\right\}
\end{aligned}
$$

A partir do logaritmo da função verossimilhança corrigida podemos obter a função escore corrigida para cada parâmetro $\beta$ e $\sigma_{e}^{2}$ derivando a expressão (2.9) com relação aos parâmetros de interesse, obtendo

$$
\mathrm{U}^{*}\left(\beta_{s}\right)=\frac{\partial \ell^{*}}{\partial \beta_{s}}\left(\beta, \sigma_{e}^{2} ; \mathbf{W}, \mathbf{Y}\right)=\frac{1}{\sigma_{e}^{2}} \sum_{i=1}^{n}\left(Y_{i} t_{i,(s)}-\sum_{j=0}^{p} \beta_{j} t_{i,(j+s)}\right),
$$

sendo $s=0,1, \ldots, p$,

$$
\begin{gathered}
\mathrm{U}^{*}\left(\sigma_{e}^{2}\right)=\frac{\partial \ell^{*}}{\partial \sigma_{e}^{2}}\left(\beta, \sigma_{e}^{2} ; \mathrm{W}, \mathrm{Y}\right)=-\frac{n}{2 \sigma_{e}^{2}}+\frac{1}{2 \sigma_{e}^{4}} \sum_{i=1}^{n}\left[Y_{i}^{2}+\sum_{j=0}^{p} \beta_{j}^{2} t_{i,(2 j)}-\right. \\
\left.-2 Y_{i} \sum_{j=0}^{p} \beta_{j} t_{i,(j)}+2 \sum_{\substack{j=0 \\
s<j}}^{p} \sum_{\substack{s=0 \\
s<j}}^{p} \beta_{j} \beta_{s} t_{i,(j+s)}\right] .
\end{gathered}
$$

b) Avaliação da função escore a partir da função escore "naive".

Neste caso consideremos o logaritmo da função verossimilhança "naive" como em (2.3), derivamos esta função em relação aos parâmetros $\beta$ e $\sigma_{e}^{2}$, obtendo as funções escores não corrigidos (ou "naive"), como proposto em Bolfarine e Gimenez (1997). As derivadas são

$$
\mathrm{U}\left(\beta_{s}\right)=\frac{\partial \ell}{\partial \beta_{s}}\left(\beta, \sigma_{e}^{2} ; \mathrm{W}, \mathrm{Y}\right)=\frac{1}{\sigma_{e}^{2}} \sum_{i=1}^{n}\left(Y_{i} W_{i}^{s}-\sum_{j=0}^{p} \beta_{j} W_{i}^{j+s}\right), \quad s=0,1, \ldots, p,
$$




$$
\begin{aligned}
\mathrm{U}\left(\sigma_{e}^{2}\right)=\frac{\partial \ell}{\partial \sigma_{e}^{2}}\left(\beta, \sigma_{e}^{2} ; \mathrm{W}, \mathrm{Y}\right)= & -\frac{n}{2 \sigma_{e}^{2}}+\frac{1}{2 \sigma_{e}^{4}} \sum_{i=1}^{n}\left[Y_{i}^{2}+\sum_{j=0}^{p} \beta_{j}^{2} W_{i}^{2 j}\right. \\
& \left.-2 Y_{i} \sum_{j=0}^{p} \beta_{j} W_{i}^{j}+2 \sum_{\substack{j=0 \\
s<j}}^{p} \sum_{\substack{s=0 \\
s<j}}^{p} \beta_{j} \beta_{s} W_{i}^{j+s}\right] .
\end{aligned}
$$

Logo, a cada função escore "naive" obtida, aplicamos o valor esperado condicional com a finalidade de obter uma função $U^{*}\left(\beta, \sigma_{e}^{2} ; \mathrm{W}, \mathrm{Y}\right)$ tal que

$$
\begin{aligned}
E\left[\mathbf{U}^{*}\left(\beta, \sigma_{e}^{2} ; \mathbf{Y}, \mathbf{W}\right)\right] & =E\left\{E\left[\mathbf{U}^{*}\left(\beta, \sigma_{e}^{2} ; \mathbf{Y}, \mathbf{W}\right) \mid \beta, \sigma_{e}^{2} ; \mathbf{Y}, \mathbf{X}\right]\right\} \\
& =E\left[\mathbf{U}\left(\beta, \sigma_{e}^{2} ; \mathbf{Y}, \mathbf{X}\right)\right] \\
& =0
\end{aligned}
$$

condição importante para obter a função escore corrigido. Então, devemos calcular

$$
\begin{gathered}
E\left(\mathbf{U}\left(\beta_{s}\right) \mid K\right)=\frac{1}{\sigma_{e}^{2}} \sum_{i=1}^{n}\left[Y_{i} E\left(W_{i}^{s} \mid K\right)-\sum_{j=0}^{p} \beta_{j} E\left(W_{i}^{j+s} \mid K\right)\right], \quad s=0,1, \ldots, p \\
E\left(\mathbf{U}\left(\sigma_{e}^{2}\right) \mid K\right)=-\frac{n}{2 \sigma_{e}^{2}}+\frac{1}{2 \sigma_{e}^{4}} \sum_{i=1}^{n}\left[Y_{i}^{2}+\sum_{j=0}^{p} \beta_{j}^{2} E\left(W_{i}^{2 j} \mid K\right)-\right. \\
\left.-2 Y_{i} \sum_{j=0}^{p} \beta_{j} E\left(W_{i}^{j} \mid K\right)+2 \sum_{j=0}^{p} \sum_{s=0}^{p} \beta_{j} \beta_{s} E\left(W_{i}^{j+s} \mid K\right)\right]
\end{gathered}
$$

Aplicando o artifício apresentado em (2.6) nas expressões (2.14) e (2.15), temos que

$$
\begin{aligned}
E\left(\mathrm{U}\left(\beta_{s}\right) \mid K\right)= & \frac{1}{\sigma_{e}^{2}} \sum_{i=1}^{n}\left[Y_{i} X_{i}^{s}-\sum_{j=0}^{p} \beta_{j} X_{i}^{s}\right] \\
& +\frac{1}{\sigma_{e}^{2}} \sum_{i=1}^{n}\left[Y_{i}\left(E\left(W_{i}^{s} \mid K\right)-X_{i}^{s}\right)-\sum_{j=0}^{p} \beta_{j}\left(E\left(W_{i}^{s} \mid K\right)-X_{i}^{s}\right)\right],
\end{aligned}
$$




$$
\begin{aligned}
E\left(\mathbf{U}\left(\sigma_{e}^{2}\right) \mid K\right)= & -\frac{n}{2 \sigma_{e}^{2}}+\frac{1}{2 \sigma_{e}^{4}}\left\{\sum _ { i = 1 } ^ { n } \left[Y_{i}^{2}+\sum_{j=0}^{p} \beta_{j}^{2} X_{i}^{2 j}-2 Y_{i} \sum_{j=0}^{p} \beta_{j} X_{i}^{j}\right.\right. \\
& \left.\left.+2 \sum_{j=0}^{p} \sum_{s=0}^{p} \beta_{j} \beta_{s} X_{i}^{j+s}\right]\right\} \\
& +\frac{1}{2 \sigma_{e}^{4}}\left\{\sum _ { i = 1 } ^ { n } \left[\sum_{j=0}^{p} \beta_{j}^{2}\left(E\left(W_{i}^{2 j} \mid K\right)-X_{i}^{2 j}\right)\right.\right. \\
& -2 Y_{i} \sum_{j=0}^{p} \beta_{j}\left(E\left(W_{i}^{j} \mid K\right)-X_{i}^{j}\right) \\
& \left.\left.+2 \sum_{j=0}^{p} \sum_{s=0}^{p} \beta_{j} \beta_{s}\left(E\left(W_{i}^{j+s} \mid K\right)-X_{i}^{j+s}\right)\right]\right\} .
\end{aligned}
$$

As expressões (2.16) e (2.17) estão compostas por duas componentes. A primeira corresponde à função escore não observado e a segunda corresponde ao vício esperado condicional da função escore não corrigido, sendo os vícios dados por:

$$
\begin{aligned}
& \operatorname{vício}\left(E\left(\mathbf{U}\left(\beta_{s}\right) \mid K\right)\right)=\frac{1}{\sigma_{e}^{2}} \sum_{i=1}^{n}\left[Y_{i}\left(E\left(W_{i}^{s} \mid K\right)-X_{i}^{s}\right)\right. \\
& \left.-\sum_{j=0}^{p} \beta_{j}\left(E\left(W_{i}^{j+s} \mid K\right)-X_{i}^{j+s}\right)\right] \\
& \operatorname{vício}\left(E\left(\mathrm{U}\left(\sigma_{e}^{2}\right) \mid K\right)\right)=\frac{1}{2 \sigma_{e}^{4}} \sum_{i=1}^{n}\left[\sum_{j=0}^{p} \beta_{j}^{2}\left(E\left(W_{i}^{2 j} \mid K\right)-X_{i}^{2 j}\right)\right. \\
& -2 Y_{i} \sum_{j=0}^{p} \beta_{j}\left(E\left(W_{i}^{j} \mid K\right)-X_{i}^{j}\right) \\
& \left.+2 \sum_{\substack{j=0 \\
s<j}}^{p} \sum_{s=0}^{p} \beta_{j} \beta_{s}\left(E\left(W_{i}^{j+s} \mid K\right)-X_{i}^{j+s}\right)\right] .
\end{aligned}
$$


Considerando a substituição de variáveis como em Cheng e Scheneeweis (1998), isto é, $E\left(W_{i}^{m} \mid K\right)$ por $W_{i}^{m}$ e $X_{i}^{m}$ por $t_{i,(m)}$, onde $t_{i,(m)}$ esta definida em (1.13), temos que

$$
\begin{aligned}
\operatorname{vício}^{*}\left(\mathbf{U}\left(\beta_{s}\right)\right) & =\frac{1}{\sigma_{e}^{2}} \sum_{i=1}^{n}\left[Y_{i}\left(W_{i}^{s}-t_{i, s}\right)-\sum_{j=0}^{p} \beta_{j}\left(W_{i}^{j+s}-t_{i,(j+s)}\right)\right], \\
\operatorname{vício}^{*}\left(\mathbf{U}\left(\sigma_{e}^{2}\right)\right)= & \frac{1}{2 \sigma_{e}^{4}} \sum_{i=1}^{n}\left[\sum_{j=0}^{p} \beta_{j}^{2}\left(W_{i}^{2 j}-t_{i,(2 j)}\right)-2 Y_{i} \sum_{j=0}^{p} \beta_{j}\left(W_{i}^{j}-t_{i,(j)}\right)\right. \\
& \left.+2 \sum_{j=0}^{p} \sum_{s=0}^{p} \beta_{j} \beta_{s}\left(W_{i}^{j+s}-t_{i,(j+s)}\right)\right]
\end{aligned}
$$

a função escore corrigido pode ser obtida a partir da seguinte expressão:

$$
\mathrm{U}^{*}(\bullet)=\mathrm{U}(\bullet)-\operatorname{vício}^{*}(\bullet)
$$

Após alguns cálculos algébricos temos as seguintes funções escores corrigidos,

$$
\begin{gathered}
\mathrm{U}^{*}\left(\beta_{s}\right)=\frac{\partial \ell^{*}}{\partial \beta_{s}}\left(\beta, \sigma_{e}^{2} ; \mathrm{Y}, \mathrm{W}\right)=\frac{1}{\sigma_{e}^{2}} \sum_{i=1}^{n}\left(Y_{i} t_{i,(s)}-\sum_{j=0}^{p} \beta_{j} t_{i,(j+s)}\right), \\
\mathrm{U}^{*}\left(\sigma_{e}^{2}\right)=\frac{\partial \ell^{*}}{\partial \sigma_{e}^{2}}\left(\beta, \sigma_{e}^{2} ; \mathrm{Y}, \mathrm{W}\right)=-\frac{n}{2 \sigma_{e}^{2}}+\frac{1}{2 \sigma_{e}^{4}} \sum_{i=1}^{n}\left[Y_{i}^{2}+\sum_{j=0}^{p} \beta_{j}^{2} t_{i,(2 j)}\right. \\
\left.-2 Y_{i} \sum_{j=0}^{p} \beta_{j} t_{i,(j)}+2 \sum_{j=0}^{p} \sum_{s=0}^{p} \beta_{j} \beta_{s} t_{i,(j+s)}\right],
\end{gathered}
$$

que coincidem com as expressões (2.10) e (2.11).

c) Elementos para a obtenção da expressão $t_{i,(m+1)}=W_{i} t_{i,(m)}-j \sigma_{u}^{2} t_{i,(m-1)}$

Como a função escore corrigido e o logaritmo da função verossimilhança consideram em sua expressão uma variável auxiliar $t_{i,(m)}$ que é função de $W_{i}^{m}$, precisamos estabelecer 
com $t_{i,(m)}$ uma relação com a variável observável $W_{i}$. Isto é feito a seguir. Para isto, primeiro precisamos relacionar $E\left(W_{i}^{m}\right) \operatorname{com} X_{i}^{m}$ já que depois utilizaremos a substituição de variáveis como em Cheng e Schneeweis (1998). Para este relacionamento recorremos ao seguinte teorema, de tal forma que $E\left(t_{i,(m)}\right)=X_{i}^{m}$, no caso do modelo funcional e $E\left(t_{i,(m)}\right)=E\left(X_{i}^{m}\right)$, no caso do modelo estrutural.

\section{Teorema 1}

Se $u_{i} \sim N\left(0, \sigma_{u}^{2}\right)$, temos de (2.2) que

$$
E\left(W_{i}^{j+1}\right)=X_{i} E\left(W_{i}^{j}\right)+j \sigma_{u}^{2} E\left(W_{i}^{j-1}\right), \quad \forall j \geq 2, i=1, \ldots, n .
$$

\section{Prova}

Como $W_{i}=X_{i}+u_{i}$, então o primeiro e segundo momentos de $W_{i}$ são definidos por $E\left(W_{i}\right)=X_{i}$ e $E\left(W_{i}^{2}\right)=X_{i}^{2}+\sigma_{u}^{2}$, devido ao fato que $u_{i} \sim N\left(0, \sigma_{u}^{2}\right)$, e para $j=2$ temos, seguindo a expressão do teorema, que

$$
\begin{aligned}
E\left(W_{i}^{3}\right) & =X_{i} E\left(W_{i}^{2}\right)+2 \sigma_{u}^{2} E\left(W_{i}\right) \\
& =X_{i}\left(X_{i}^{2}+\sigma_{u}^{2}\right)+2 \sigma_{u}^{2} X_{i} \\
& =X_{i}^{3}+3 X_{i} \sigma_{u}^{2}
\end{aligned}
$$

Se usamos a expressão (2.2) para obter o terceiro momento de $W_{i}$, temos que

$$
\begin{aligned}
E\left(W_{i}^{3}\right) & =E\left(X_{i}+u_{i}\right)^{3} \\
& =\sum_{q=0}^{3}\left(\begin{array}{l}
3 \\
q
\end{array}\right) X_{i}^{q} E\left(u_{i}^{3-q}\right) \\
& =X_{i}^{3}+3 X_{i}^{2} E\left(u_{i}\right)+3 X_{i} E\left(u_{i}^{2}\right)+E\left(u_{i}^{3}\right),
\end{aligned}
$$

e como $u_{i} \sim N\left(0, \sigma_{u}^{2}\right)$, então os momentos ímpares da variável $u_{i}$ são todos iguais a zero, de modo que

$$
E\left(W_{i}^{3}\right)=X_{i}^{3}+3 X_{i} \sigma_{u}^{2}
$$

Notamos que as equações (2.20) e (2.21) são iguais. Isto permite-nos concluir que a expressão do teorema 1 produz o terceiro momento da variável $W_{i}$ corretamente.

Para o caso geral, temos que demonstrar que a expressão definida no teorema 1 leva a expressão seguinte 


$$
E\left(W_{i}^{j+1}\right)=E\left(X_{i}+u_{i}\right)^{j+1}=\sum_{q=0}^{j+1}\left(\begin{array}{c}
j+1 \\
q
\end{array}\right) X_{i}^{q} E\left(u_{i}^{j+1-q}\right)
$$

Substituindo a expressão (2.22) nas somas no lado direito da equação do teorema 1 temos que

$$
E\left(W_{i}^{j+1}\right)=X_{i} \sum_{q=0}^{j}\left(\begin{array}{l}
j \\
q
\end{array}\right) X_{i}^{q} E\left(u_{i}^{j-q}\right)+j \sigma_{u}^{2} \sum_{q=0}^{j-1}\left(\begin{array}{c}
j-1 \\
q
\end{array}\right) X_{i}^{q} E\left(u_{i}^{j-1-q}\right), \forall j \geq 2 .
$$

Com a finalidade de simplificar a expressão (2.24), especificamente o segundo somando da expressão, fazemos uso da seguinte proposição:

\section{Proposição 1}

Se $H_{i} \sim N\left(0, \sigma_{h}^{2}\right)$, a função geradora de momentos da variável $H_{i}$ é $M_{H_{i}}(t)=\exp \frac{1}{2} \sigma_{h}^{2} t^{2}$ valendo a seguinte relação:

$$
\frac{\partial^{(j+1)}}{\partial t^{(j+1)}} M_{H_{i}}(t)=t \sigma_{h}^{2} \frac{\partial^{(j)}}{\partial t^{(j)}} M_{H_{i}}(t)+j \sigma_{h}^{2} \frac{\partial^{(j-1)}}{\partial t^{(j-1)}} M_{H_{i}}(t)
$$

para $j \geq 2$, onde $\partial t^{(\mathrm{q})}$ denota a q-ésima derivada parcial em relação a $t$.

Usando a relação da proposição 1 para obter o q-ésimo momento da variável $H_{i}$ quando $t=0$, temos

$$
E\left(H_{i}^{q}\right)=\frac{\partial^{(q)}}{\partial t^{(q)}} M_{H_{i}}(0)=(q-1) \sigma_{h}^{2} E\left(H_{i}^{q-2}\right) .
$$

Daqui podemos concluir que

$$
\sigma_{h}^{2} E\left(H_{i}^{s-2}\right)=\frac{E\left(H_{i}^{s}\right)}{s-1}, \quad s \geq 4 .
$$

Aplicando (2.24) no segundo somando da expressão (2.23) temos

$$
\begin{aligned}
E\left(W^{j+1}\right) & =X_{i} \sum_{q=0}^{j}\left(\begin{array}{l}
j \\
q
\end{array}\right) X_{i}^{q} E\left(u_{i}^{j-q}\right)+j \sum_{q=0}^{j-1}\left(\begin{array}{c}
j-1 \\
q
\end{array}\right) X_{i}^{q} \frac{E\left(u^{j+1-q}\right)}{j-q} \\
& =\sum_{q=0}^{j}\left(\begin{array}{l}
j \\
q
\end{array}\right) X_{i}^{q+1} E\left(u_{i}^{j-q}\right)+\sum_{q=0}^{j}\left(\begin{array}{l}
j \\
q
\end{array}\right) X_{i}^{q} E\left(u_{i}^{j+1-q}\right) .
\end{aligned}
$$

Desenvolvendo os somatórios temos 


$$
\begin{aligned}
E\left(W^{j+1}\right)= & \left(\begin{array}{l}
j \\
0
\end{array}\right) X_{i}^{0} E\left(U_{i}^{j+1}\right)+\left[\left(\begin{array}{l}
j \\
0
\end{array}\right)+\left(\begin{array}{l}
j \\
1
\end{array}\right)\right] X_{i} E\left(U_{i}^{j}\right) \\
& +\ldots+\left[\left(\begin{array}{c}
j \\
j-1
\end{array}\right)+\left(\begin{array}{l}
j \\
j
\end{array}\right)\right] X_{i}^{j} E\left(u_{i}\right)+\left(\begin{array}{l}
j \\
j
\end{array}\right) X_{i}^{j+1} E\left(u_{i}^{0}\right) .
\end{aligned}
$$

Como

$$
\left(\begin{array}{c}
k \\
q-1
\end{array}\right)+\left(\begin{array}{l}
k \\
q
\end{array}\right)=\left(\begin{array}{c}
k+1 \\
q
\end{array}\right), \quad\left(\begin{array}{l}
k \\
0
\end{array}\right)=\left(\begin{array}{c}
k+1 \\
0
\end{array}\right) \quad \text { e } \quad\left(\begin{array}{l}
k \\
k
\end{array}\right)=\left(\begin{array}{l}
k+1 \\
k+1
\end{array}\right),
$$

chegamos à expressão (2.22). Deste modo fica provado o teorema 1.

Substituindo $X_{i}$ por $t_{i,(1)}$ e $E\left(W_{i}^{j}\right)$ por $W_{i}^{j}$, podemos escrever a expressão do teorema 1 como

$$
W_{i}^{j+1}=t_{i,(1)} W_{i}^{j}+j \sigma_{u}^{2} W_{i}^{j-1}, i=1, \ldots, n .
$$

A relação acima estabelece $W_{i}^{m}$ em função de $t_{i,(m)}$, mas precisamos a relação inversa, isto é, $t_{i,(m)}$ em função de $W_{i}^{m}$ com a finalidade de ter os valores apropriados para a função escore corrigido que está em função de $t_{i,(m)}$. Para isto enunciamos o teorema 2.

\section{Teorema 2}

Se $u_{i} \sim N\left(0, \sigma_{u}^{2}\right)$ e se (2.2) for válida, temos que

$$
X_{i}^{j+1}=E\left(W_{i}\right) X_{i}^{j}-j \sigma_{u}^{2} X_{i}^{j-1}, \quad \forall j \geq 2
$$

\section{Prova}

A expressão do teorema 1 pode ser escrito como

$$
X_{i} E\left(W_{i}^{j}\right)=E\left(W_{i}^{j+1}\right)-j \sigma_{u}^{2} E\left(W_{i}^{j-1}\right)
$$

Substituindo a expressão (2.23) na expressão acima obtemos

$$
\begin{aligned}
X_{i}\left[\sum_{q=0}^{j}\left(\begin{array}{l}
j \\
q
\end{array}\right) X_{i}^{j-q} E\left(u_{i}^{q}\right)\right]= & {\left[\sum_{q=0}^{j+1}\left(\begin{array}{c}
j+1 \\
q
\end{array}\right) X_{i}^{j+1-q} E\left(u^{q}\right)\right] } \\
& -j \sigma_{u}^{2}\left[\sum_{q=0}^{j-1}\left(\begin{array}{c}
j-1 \\
q
\end{array}\right) X_{i}^{j-1-q} E\left(u_{i}^{q}\right)\right],
\end{aligned}
$$

considerando $X_{i}=E\left(W_{i}\right)$ no primeiro somando do lado direito da expressão (2.25), temos 


$$
\begin{aligned}
\sum_{q=0}^{j}\left(\begin{array}{l}
j \\
q
\end{array}\right) X_{i}^{j+1-q} E\left(u_{i}^{q}\right)= & \sum_{q=0}^{j+1}\left(\begin{array}{c}
j+1 \\
q
\end{array}\right) E\left(W_{i}\right) X_{i}^{j-q} E\left(u_{i}^{q}\right) \\
& -\sum_{q=0}^{j-1}\left(\begin{array}{c}
j-1 \\
q
\end{array}\right) j \sigma_{u}^{2} X_{i}^{j-1-q} E\left(u_{i}^{q}\right) .
\end{aligned}
$$

A relação acima permite gerar a expressão seguinte

$$
X_{i}^{j+1}=E\left(W_{i}\right) X_{i}^{j}-j \sigma_{u}^{2} X_{i}^{j-1}
$$

esta expressão é valida para qualquer valor $j>2$.

Considerando a expressão do teorema e as substituições de variáveis como em Cheng e Schneweeis (1998), temos que a expressão em (2.26) passa a ser

$$
t_{i,(j+1)}=W_{i} t_{i,(j)}-j \sigma_{u}^{2} t_{i,(j-1)}, \quad j>2 .
$$

Com isto podemos ter a expressão da função escore corrigido em função de $W_{i}$ e $\sigma_{u}^{2}$, fazendo $t_{i,(0)}=1$.

A técnica desenvolvida por Nakamura (1990) e Bolfarine e Gimenez (1997) é válida tanto no modelo funcional quanto no modelo estrutural.

Para o modelo estrutural a forma do teorema 1 muda um pouco, sendo esta como no teorema 3.

\section{Teorema 3}

Se $u_{i} \sim N\left(0, \sigma_{u}^{2}\right)$, e $X_{i} \sim N\left(\mu_{X}, \sigma_{X}^{2}\right), i=1, \ldots, n$, temos a seguinte relação

$$
E\left(W_{i}^{j+1}\right)=\mu_{X} E\left(W_{i}^{j}\right)+j\left(\sigma_{u}^{2}+\sigma_{X}^{2}\right) E\left(W_{i}^{j-1}\right), \quad \forall j \geq 2 .
$$

\section{Prova}

Similar à prova do teorema 1 .

No modelo estrutural a substituição de variáveis é um pouco diferente do modelo funcional apresentado por Cheng e Schneeweis (1998), sendo as substituições para o 
modelo estrutural as seguintes: $E\left(W_{i}^{m}\right)$ por $W_{i}^{m}$ e $E\left(X_{i}^{m}\right)$ por $t_{i,(m)}$, sendo a relação de $t_{i,(m)}$ como função de $W_{i}$ a mesma que na expressão (2.27).

d) Observações.

- Considerando as funções escores corrigidas das expressões (2.18) e (2.19) podemos obter os seguintes estimadores dos parâmetros $\beta$ e $\sigma_{\mathbf{e}}^{2}$, os quais tem expressões fechadas, quando $\sigma_{u}^{2}$ é conhecido.

$$
\begin{gathered}
\left(\begin{array}{cccc}
n & \sum_{i=1}^{n} t_{i,(1)} & \cdots & \sum_{i=1}^{n} t_{i,(p)} \\
\sum_{i=1}^{n} t_{i,(1)} & \sum_{i=1}^{n} t_{i,(2)} & \cdots & \sum_{i=1}^{n} t_{i,(p+1)} \\
\vdots & \vdots & \cdots & \vdots \\
\sum_{i=1}^{n} t_{i,(p)} & \sum_{i=1}^{n} t_{i,(p+1)} & \cdots & \sum_{i=1}^{n} t_{i,(2 p)}
\end{array}\right)\left(\begin{array}{c}
\hat{\beta}_{0} \\
\hat{\beta}_{1} \\
\vdots \\
\hat{\beta}_{p}
\end{array}\right)=\left(\begin{array}{c}
\sum_{i=1}^{n} Y_{i} \\
\sum_{i=1}^{n} Y_{i} t_{i,(1)} \\
\vdots \\
\sum_{i=1}^{n} Y_{i} t_{i,(p)}
\end{array}\right) \\
\hat{\sigma}_{e}^{2}=\frac{1}{n} \sum_{i=1}^{n}\left[Y_{i}^{2}+\sum_{j=0}^{p} \hat{\beta}_{j}^{2} t_{i,(2 j)}-2 Y_{i} \sum_{j=0}^{p} \hat{\beta}_{j} t_{i,(j)}+\right. \\
\left.+2 \sum_{j=0}^{p} \sum_{s=0}^{p} \hat{\beta}_{j} \hat{\beta}_{s} t_{i,(j+s)}\right] .
\end{gathered}
$$

- A metodologia apresentada é válida tanto para modelos funcionais como para modelos estruturais.

- Esta metodologia se baseia em uma relação de recursividade para a obtenção dos estimadores.

- A metodologia proposta por Nakamura (1990) e a metodologia proposta por Bolfarine e Gimenez (1997) aplicadas aos modelos polinomiais com erros nas variáveis são coincidentes quando $\sigma_{u}^{2}$ é considerado conhecida. 
- Os estimadores obtidos a partir de um modelo polinomial com erros nas variáveis usando o logaritmo da função verossimilhança corrigida Nakamura, (1990) ou a função escore corrigido Bolfarine e Gimenez, (1997) coincidem com os estimadores de mínimos quadrados ajustados (Cheng e Schneeweis, (1998)) quando $\sigma_{u}^{2}$ é conhecida.

- Para este caso ( $\sigma_{u}^{2}$ conhecida) a condição fundamental da metodologia desenvolvida por Nakamura (1990), que é, $E(\bullet \mid \mathrm{Y}, \mathrm{X})$ e $\frac{\partial}{\partial \theta}(\bullet)$ serem permutáveis, é satisfeita.

e) Exemplos.

\section{Polinômio de primeiro grau}

Considerando a expressão (2.28) e (2.29), para $\mathrm{p}=1$ temos

$$
\begin{gathered}
\left(\begin{array}{cc}
n & \sum_{i=1}^{n} t_{i,(1)} \\
\sum_{i=1}^{n} t_{i,(1)} & \sum_{i=1}^{n} t_{i,(2)}
\end{array}\right)\left(\begin{array}{l}
\hat{\beta}_{0} \\
\hat{\beta}_{1}
\end{array}\right)=\left(\begin{array}{c}
\sum_{i=1}^{n} Y_{i} \\
\sum_{i=1}^{n} Y_{i} t_{i,(1)}
\end{array}\right), \\
\hat{\sigma}_{e}^{2}=\frac{1}{n} \sum_{i=1}^{n}\left[Y_{i}^{2}+\hat{\beta}_{0}^{2}+\hat{\beta}_{1}^{2} t_{i,(2)}-2 Y_{i} \hat{\beta}_{0}-2 Y_{i} \hat{\beta}_{1} t_{i,(1)}+2 \hat{\beta}_{0} \hat{\beta}_{1} t_{i,(1)}\right] .
\end{gathered}
$$

Após algumas manipulações algébricas temos

$$
\begin{aligned}
& \hat{\beta}_{0}=\frac{\sum_{i=1}^{n} Y_{i}}{n}-\frac{\sum_{i=1}^{n} t_{i,(1)}}{n} \hat{\beta}_{1}, \\
& \hat{\beta}_{1}=\frac{n \sum_{i=1}^{n} Y_{i} t_{i,(1)}-\sum_{i=1}^{n} t_{i,(1)} \sum_{i=1}^{n} Y_{i}}{n \sum_{i=1}^{n} t_{i,(2)}-\left(\sum_{i=1}^{n} t_{i,(1)}\right)^{2}} .
\end{aligned}
$$

Levando em conta (2.27) temos que

$$
\begin{aligned}
\sum_{i=1}^{n} t_{i,(1)} & =\sum_{i=1}^{n} W_{i} \\
\sum_{i=1}^{n} t_{i,(2)} & =\sum_{i=1}^{n} W_{i}^{2}-n \sigma_{u}^{2}=n S_{W W}+\frac{1}{n}\left(\sum_{i=1}^{n} W_{i}\right)^{2}-n \sigma_{u}^{2}, \\
\sum_{i=1}^{n} Y_{i} t_{i,(1)} & =\sum_{i=1}^{n} Y_{i} W_{i}=n S_{W Y}+\frac{1}{n}\left(\sum_{i=1}^{n} Y_{i}\right)\left(\sum_{i=1}^{n} W_{i}\right)
\end{aligned}
$$


onde

$$
\begin{aligned}
& n S_{W W}=\sum_{i=1}^{n} W_{i}^{2}-\frac{1}{n}\left(\sum_{i=1}^{n} W_{i}\right)^{2} \\
& n S_{Y W}=\sum_{i=1}^{n} Y_{i} W_{i}-\frac{1}{n}\left(\sum_{i=1}^{n} Y_{i}\right)\left(\sum_{i=1}^{n} W_{i}\right)
\end{aligned}
$$

logo,

$$
\begin{gathered}
\hat{\beta}_{0}=\bar{Y}-\bar{W} \hat{\beta}_{1}, \\
\hat{\beta}_{1}=\frac{S_{Y W}}{S_{W W}-\sigma_{u}^{2}}, \\
\hat{\sigma}_{e}^{2}=S_{Y Y}+\hat{\beta}_{1}\left[\hat{\beta}_{1}\left(S_{W W}-\sigma_{u}^{2}\right)-2 S_{Y W}\right] .
\end{gathered}
$$

Os estimadores em (2.30) e (2.31) são similares aos obtidos pelo Bolfarine e Gimenez (1997), para $p=1$.

\section{Polinômio de segundo grau}

Considerando as expressão (2.28) e (2.29) para $\mathrm{p}=2$, temos

$$
\begin{aligned}
& \left(\begin{array}{ccc}
n & \sum_{i=1}^{n} t_{i,(1)} & \sum_{i=1}^{n} t_{i,(2)} \\
\sum_{i=1}^{n} t_{i,(1)} & \sum_{i=1}^{n} t_{i,(2)} & \sum_{i=1}^{n} t_{i,(3)} \\
\sum_{i=1}^{n} t_{i,(2)} & \sum_{i=1}^{n} t_{i,(3)} & \sum_{i=1}^{n} t_{i,(4)}
\end{array}\right)\left(\begin{array}{l}
\hat{\beta}_{0} \\
\hat{\beta}_{1} \\
\hat{\beta}_{2}
\end{array}\right)=\left(\begin{array}{c}
\sum_{i=1}^{n} Y_{i} \\
\sum_{i=1}^{n} Y_{i} t_{i,(1)} \\
\sum_{i=1}^{n} Y_{i} t_{i,(2)}
\end{array}\right), \\
& \hat{\sigma}_{e}^{2}=\frac{1}{n} \sum_{i=1}^{n}\left[Y_{i}^{2}+\hat{\beta}_{0}^{2}+\hat{\beta}_{1}^{2} t_{i,(2)}+\hat{\beta}_{2}^{2} t_{i,(4)}-2 Y_{i} \hat{\beta}_{0}-2 Y_{i} \hat{\beta}_{1} t_{i,(1)}\right. \\
& \left.-2 Y_{i} \hat{\beta}_{2} t_{i,(2)}+2 \hat{\beta}_{0} \hat{\beta}_{1} t_{i,(1)}+2 \hat{\beta}_{0} \hat{\beta}_{2} t_{i,(2)}+2 \hat{\beta}_{1} \hat{\beta}_{2} t_{i,(3)}\right] .
\end{aligned}
$$

Considerando os seguintes desvios: 


$$
\begin{aligned}
& A=n \sum_{i=1}^{n} Y_{i} t_{i,(1)}-\left(\sum_{i=1}^{n} Y_{i} \sum_{i=1}^{n} t_{i,(1)}\right), \\
& B=n \sum_{i=1}^{n} Y_{i} t_{i,(2)}-\left(\sum_{i=1}^{n} Y_{i} \sum_{i=1}^{n} t_{i,(2)}\right), \\
& C=n \sum_{i=1}^{n} t_{i,(2)}-\left(\sum_{i=1}^{n} t_{i,(1)}\right)^{2}, \\
& D=n \sum_{i=1}^{n} t_{i,(3)}-\left(\sum_{i=1}^{n} t_{i,(1)}\right)\left(\sum_{i=1}^{n} t_{i,(2)}\right), \\
& E=n \sum_{i=1}^{n} t_{i,(4)}-\left(\sum_{i=1}^{n} t_{i,(2)}\right)^{2},
\end{aligned}
$$

após algumas manipulações algébricas temos

$$
\begin{gathered}
\hat{\beta}_{0}=\frac{1}{n} \sum_{i=1}^{n} Y_{i}-\frac{1}{n} \sum_{i=1}^{n} t_{i,(1)} \hat{\beta}_{1}-\frac{1}{n} \sum_{i=1}^{n} t_{i,(2)} \hat{\beta}_{2}, \\
\hat{\beta}_{1}=\frac{1}{C}\left(A-D \hat{\beta}_{2}\right), \\
\hat{\beta}_{2}=\frac{C B-D A}{E C-D^{2}} .
\end{gathered}
$$

Levando em conta (2.27) segue $t_{i,(1)}=W_{i}, \quad t_{i,(2)}=W_{i}^{2}-\sigma_{u}^{2}, \quad t_{i,(3)}=W_{i}^{3}-3 W_{i} \sigma_{u}^{2}$ e $t_{i,(4)}=W_{i}^{4}-6 W_{i}^{2} \sigma_{u}^{2}+3 \sigma_{u}^{4}$, de forma que os desvios podem ser expressos da seguinte maneira:

$$
\begin{aligned}
& A=n^{2} S_{Y W}, \quad B=n^{2} S_{Y W^{2}}, \quad C=n^{2} S_{W W}-n^{2} \sigma_{u}^{2}, \\
& D=n^{2} S_{W W^{2}}-2 n \sum_{i=1}^{n} W_{i} \sigma_{u}^{2} \quad e \\
& E=n^{2} S_{W^{2} W^{2}}-4 n^{2} \sigma_{u}^{2} S_{W W}-4 \sigma_{u}^{2}\left(\sum_{i=1}^{n} W_{i}\right)^{2}+4 n^{2} \sigma_{u}^{4} .
\end{aligned}
$$

Com estas novas expressões de A, B, C, D e E, podemos reescrever as expressões de $\hat{\beta}_{0}, \hat{\beta}_{1}$ e $\hat{\beta}_{2}$. O estimador de $\sigma_{e}^{2}$, é obtido a partir de 


$$
\begin{aligned}
\hat{\sigma}_{e}^{2}= & \left(S_{Y Y}+S_{W W} \hat{\beta}_{1}^{2}+S_{W^{2} W^{2}} \hat{\beta}_{2}^{2}-2 S_{Y W} \hat{\beta}_{1}^{2}-2 S_{Y W^{2}} \hat{\beta}_{2}^{2}-2 S_{W W^{2}} \hat{\beta}_{1}^{2} \hat{\beta}_{2}^{2}\right)- \\
& -\left(4 \hat{\beta}_{2}^{2} S_{W W}-\hat{\beta}_{1}^{2}+4 \hat{\beta}_{1} \hat{\beta}_{2} \bar{W}+4 \hat{\beta}_{2}^{2} \bar{W}^{2}\right) \sigma_{u}^{2}+2 \hat{\beta}_{2}^{2} \sigma_{u}^{4},
\end{aligned}
$$

onde

$$
\begin{aligned}
n^{2} S_{W^{2} W^{2}} & =n \sum_{i=1}^{n} W_{i}^{4}-\left(\sum_{i=1}^{n} W_{i}^{2}\right)^{2} \\
n^{2} S_{W W^{2}} & =n \sum_{i=1}^{n} W_{i}^{3}-\left(\sum_{i=1}^{n} W_{i}\right)\left(\sum_{i=1}^{n} W_{i}^{2}\right), \\
n^{2} S_{W W} & =n \sum_{i=1}^{n} W_{i}^{2}-\left(\sum_{i=1}^{n} W_{i}\right)^{2} \\
n^{2} S_{Y W^{2}} & =n \sum_{i=1}^{n} Y_{i} W_{i}^{2}-\left(\sum_{i=1}^{n} Y_{i}\right)\left(\sum_{i=1}^{n} W_{i}^{2}\right) \\
n^{2} S_{Y W} & =n \sum_{i=1}^{n} Y_{i} W_{i}-\left(\sum_{i=1}^{n} Y_{i}\right)\left(\sum_{i=1}^{n} W_{i}\right) .
\end{aligned}
$$

\subsubsection{Obtenção da matriz de covariâncias assintóticas}

No trabalho de Nakamura (1990) não foram apresentados as condições de regularidade para a validade dos resultados assintóticos, assumindo-se que a teoria de $\mathrm{M}$-estimador ( $\mathrm{Hu}$ ber, 1981) pode ser aplicada.

No trabalho de Bolfarine e Gimenes (1997) foram apresentadas as condições de regularidade que validam os resultados assintóticos, eles afirmam que se deve ter um tratamento rigoroso das condições de regularidade para o modelo funcional, já que a suposição de observações identicamente distribuídas não são verificadas. Isto é, para cada i, a distribuição $\left(W_{i}, Y_{i}\right)$ dependerá do parâmetro estrutural $\theta$ e do parâmetro incidental $X_{i}$, porem qualquer convergência aos verdadeiros valores dependerá do comportamento assintótico assumido para a seqüencia de parâmetros incidentais $\left(X_{i}\right)$. Desta forma, as condições de regularidade impostas serão bastante diferentes das usuais no caso iid.

Bolfarine e Gimenes (1997) apresentam as condições de unicidade considerando as condições de regularidade, assim como a normalidade assintótica que são igualmente 
válidas para nosso estudo, eles demonstraram que o estimador da matriz de covariâncias assintóticas tem a forma da expressão (1.21), deste modo nossa matriz de covariâncias assintóticas tem a mesma forma que a expressão referida, sendo esta

$$
£^{*}(\beta ; \mathrm{W}, \mathrm{Y})=\frac{1}{n}\left(\mathbf{I}^{*}(\beta ; \mathrm{W}, \mathrm{Y})\right)^{-1} \mathbf{S}^{*}(\beta ; \mathrm{W}, \mathrm{Y})\left(\mathbf{I}^{*}(\beta ; \mathrm{W}, \mathrm{Y})\right)^{-1}
$$

sendo

$$
\mathbf{I}^{*}(\hat{\beta} ; \mathrm{W}, \mathrm{Y})=-\frac{1}{n} \sum_{i=1}^{n} \frac{\partial}{\partial \beta} U_{i}^{*}(\hat{\beta} ; \mathrm{W}, \mathrm{Y})
$$

e

$$
\mathrm{S}^{*}(\hat{\beta} ; \mathrm{W}, \mathrm{Y})=\frac{1}{n} \sum_{i=1}^{n} \mathrm{U}_{i}^{*}(\hat{\beta} ; \mathrm{W}, \mathrm{Y}) \mathrm{U}_{i}^{*}(\hat{\beta} ; \mathrm{W}, \mathrm{Y})^{t}
$$

a partir disto temos

$$
\sqrt{n}(\hat{\beta}-\beta) \stackrel{D}{\longrightarrow} N_{p+1}\left(0, £^{*}\right)
$$

\subsubsection{Teste de hipóteses para $\beta$}

Bolfarine e Gimenes (1997) estudaram os testes apropriados para uma função escore corrigido qualquer, nos apresentaremos a adequação desse estudo ao caso polinomial, consideremos para esta situação que

$$
\begin{gathered}
U^{*}(\theta)=\left(\begin{array}{c}
U^{*}\left(\beta_{0}\right) \\
U^{*}\left(\beta_{1}\right) \\
\vdots \\
U^{*}\left(\beta_{p}\right) \\
U^{*}\left(\sigma_{e}^{2}\right)
\end{array}\right)=\left(\begin{array}{c}
U^{*}(\beta) \\
U^{*}\left(\sigma_{e}^{2}\right)
\end{array}\right), \\
I^{*}(\theta)=\left(\begin{array}{ccc}
-\frac{\partial}{\partial \beta_{0}} U^{*}\left(\beta_{1}\right)-\frac{\partial}{\partial \beta_{1}} U^{*}\left(\beta_{1}\right) \cdots-\frac{\partial}{\partial \beta_{p}} U^{*}\left(\beta_{1}\right)-\frac{\partial}{\partial \sigma_{e}^{2}} U^{*}\left(\beta_{1}\right) \\
\vdots \\
\vdots \\
-\frac{\partial}{\partial \beta_{0}} U^{*}\left(\beta_{p}\right)-\frac{\partial}{\partial \beta_{1}} U^{*}\left(\beta_{p}\right) \cdots-\frac{\partial}{\partial \beta_{p}} U^{*}\left(\beta_{p}\right)-\frac{\partial}{\partial \sigma_{e}^{2}} U^{*}\left(\beta_{p}\right) \\
-\frac{\partial}{\partial \beta_{0}} U^{*}\left(\sigma_{e}^{2}\right)-\frac{\partial}{\partial \beta_{1}} U^{*}\left(\sigma_{e}^{2}\right) \cdots-\frac{\partial}{\partial \beta_{p}} U^{*}\left(\sigma_{e}^{2}\right)-\frac{\partial}{\partial \sigma_{e}^{2}} U^{*}\left(\sigma_{e}^{2}\right)
\end{array}\right) \\
=\left(\begin{array}{c}
-\frac{\partial}{\partial \beta} U^{*}(\beta)-\frac{\partial}{\partial \sigma_{e}^{2}} U^{*}(\beta) \\
-\frac{\partial}{\partial \beta} U^{*}\left(\sigma_{e}^{2}\right)-\frac{\partial}{\partial \sigma_{e}^{2}} U^{*}\left(\sigma_{e}^{2}\right)
\end{array}\right) \cdot
\end{gathered}
$$


A partir dessas expressões podemos considerar que $\bar{\Lambda}_{n}(\theta)=\frac{1}{n} E\left[I^{*}(\theta)\right]$, onde $\theta=\left(\beta^{t}, \sigma_{e}^{2}\right)^{t}$ e $\bar{\Gamma}_{n}(\theta)=\frac{1}{n} \sum_{i=1}^{n} E\left[U_{i}^{*}(\theta) U_{i}^{*}(\theta)^{t}\right]$, quando n é grande as matrices acima apresentadas, convergem para as matrizes positivas definidas $\Lambda(\theta)$ e $\Gamma(\theta)$. Como nosso interesse é testar a hipóteses nula $H_{0}: \beta=\beta^{0}$ na presença do parâmetro "nuisance" $\sigma_{e}^{2}$, as matrizes particionadas segundo as dimensões de $\sigma_{e}^{2}$ e $\beta$ são

$$
\Lambda(\theta)=\left(\begin{array}{cc}
\Lambda_{\beta \beta}(\theta) & \Lambda_{\beta \sigma_{e}^{2}}(\theta) \\
\Lambda_{\sigma_{e}^{2} \beta}(\theta) & \Lambda_{\sigma_{e}^{2} \sigma_{e}^{2}}(\theta)
\end{array}\right) \quad \Gamma(\theta)=\left(\begin{array}{cc}
\Gamma_{\beta \beta}(\theta) & \Gamma_{\beta \sigma_{e}^{2}}(\theta) \\
\Gamma_{\sigma_{e}^{2} \beta}(\theta) & \Gamma_{\sigma_{e}^{2} \sigma_{e}^{2}}(\theta)
\end{array}\right),
$$

onde as matrizes $\Lambda_{\beta \beta}(\theta)$ ou $\Gamma_{\beta \beta}(\theta)$ são de dimensão $(p+1) \times(p+1)$, os vetores $\Lambda_{\beta \sigma_{e}^{2}}(\theta)$ ou $\Gamma_{\beta \sigma_{e}^{2}}(\theta)$ são de dimensão $1 \times(p+1)$ e o escalar $\Lambda_{\sigma_{e}^{2} \sigma_{e}^{2}}(\theta)$ ou $\Gamma_{\sigma_{e}^{2} \sigma_{e}^{2}}(\theta)$ são de dimensão $1 \times 1$.

Sejam $\hat{\theta}=\left(\hat{\beta}^{t}, \hat{\sigma}_{e}^{2}\right)^{t}$ e $\hat{\theta}_{0}=\left(\hat{\beta}_{0}^{t}, \hat{\sigma}_{e}^{2}\right)^{t}$ estimadores consistentes de $\theta$, satisfazendo $U^{*}(\hat{\theta})=0$ e $U^{*}(\hat{\beta})=0$, ou seja, $\hat{\theta}$ e $\hat{\theta}_{0}$ são estimadores corrigidos irrestritos e restritos a $H_{0}$, respectivamente. Os testes de Wald e Escore que podem ser aplicados são

$$
\begin{gathered}
W=n\left(\hat{\beta}-\beta^{0}\right)^{t} \hat{\Lambda}_{\beta \beta . \sigma_{e}^{2}}\left(\hat{\theta}_{0}\right)\left(\hat{\beta}-\beta^{0}\right), \\
Q=\frac{1}{n} U_{\beta}^{*}\left(\hat{\theta}_{0}\right)^{t} \hat{\Lambda}_{\beta \beta . \sigma_{e}^{2}}^{-1}\left(\hat{\theta}_{0}\right) U_{\beta}^{*}\left(\hat{\theta}_{0}\right), \\
W_{c}=n\left(\hat{\beta}-\beta^{0}\right)^{t} \hat{\Omega}_{\beta \beta}^{-1}\left(\hat{\theta}_{0}\right)\left(\hat{\beta}-\beta^{0}\right),
\end{gathered}
$$

sendo

$$
\hat{\Lambda}_{\beta \beta . \sigma_{e}^{2}}\left(\hat{\theta}_{0}\right)=\hat{\Lambda}_{\beta \beta}\left(\hat{\theta}_{0}\right)-\hat{\Lambda}_{\sigma_{e}^{2} \sigma_{e}^{2}}^{-1}\left(\hat{\theta}_{0}\right) \hat{\Lambda}_{\beta \sigma_{e}^{2}}\left(\hat{\theta}_{0}\right)
$$

e

assim também

$$
\hat{\Omega}_{\beta \beta}\left(\hat{\theta}_{0}\right)=\left(\hat{\Lambda}^{-1}\left(\hat{\theta}_{0}\right) \hat{\Gamma}\left(\hat{\theta}_{0}\right) \hat{\Lambda}^{-1}\left(\hat{\theta}_{0}\right)\right)_{\beta \beta}
$$

$$
Q_{c}=\frac{1}{n} U_{\beta}^{*}\left(\hat{\theta}_{0}\right)^{t} \hat{\Lambda}_{\beta \beta . \sigma_{e}^{2}}^{-1}\left(\hat{\theta}_{0}\right) \hat{\Omega}_{\beta \beta}^{-1}\left(\hat{\theta}_{0}\right) \hat{\Lambda}_{\beta \beta . \sigma_{e}^{2}}^{-1}\left(\hat{\theta}_{0}\right) U_{\beta}^{*}\left(\hat{\theta}_{0}\right) .
$$

Quando a função escore corrigido corresponde a gradiente do logaritmo da função de verossimilhança corrigida $\ell^{*}(\theta)$, isto é,

$$
U^{*}(\theta)=\frac{\partial \ell^{*}(\theta)}{\partial \theta}
$$

é possível definir um teste de razão de verossimilhança corrigida. A estatística de razão de verossimilhança corrigida é definida 


$$
L=2 \ell^{*}(\hat{\theta})-\ell^{*}\left(\hat{\theta}_{0}\right)
$$

Bolfarine e Gimenes (1997) demonstraram que os testes (2.38), (2.39) e (2.42) tem a mesma distribuição assintótica sob a hipóteses nula, assim também demonstraram que os testes (2.40) e (2.41) também são assintoticamente equivalentes.

\subsubsection{Aplicações numéricas.}

Para avaliar a metodologia apresentada consideramos duas situações, uma ș̣mulação de Monte Carlo e um conjunto de dados reais.

\section{a. Um estudo de simulação}

Consideramos para nossa simulação o modelo funcional em um polinômio de grau dois. efetuamos 1.000 replicações para estudar a convergência dos estimadores e os seguintes parâmetros são tomados em consideração:

1. Para $n$ especificado, selecionamos $X_{i} \sim \operatorname{Binomial}(n ; 0,5)$, independente $u_{i} \sim N(0 ; 0,05)$ independentes e $e_{i} \sim N(0 ; 1)$ independentes para $i=1,2, \ldots, n$.

2. Obtenção dos dados:

$$
\begin{aligned}
W_{i} & =X_{i}+u_{i}, \\
Y_{i} & =\beta_{0}+\beta_{1} X_{i}+\beta_{2} X_{i}^{2}+e_{i}, \quad i=1, \ldots, n
\end{aligned}
$$

sendo $\beta_{0}=\beta_{1}=\beta_{2}=1$.

3. Para efeitos práticos, deve-se considerar as expressões (2.33)-(2.36), para a estimação dos parâmetros.

\section{Resultados}

Em seguida apresentamos os resultados obtidos da simulação implementada acima em linguagem OX (Doornik, 1998) 
Tabela 2.1. Estimativas segundo os métodos "naive"e "escore corrigido"para diferentes tamanhos de amostra.

\begin{tabular}{|c|c|c|c|c|c|c|c|}
\hline \multirow[t]{2}{*}{$\mathrm{n}$} & \multirow[t]{2}{*}{ Parâmetro } & \multicolumn{3}{|c|}{ Naive } & \multicolumn{3}{|c|}{ Escore corrigido } \\
\hline & & Estimativa & Desv.Pad. & EQM & Estimativa & Desv.Pad. & EQM \\
\hline \multirow{4}{*}{500} & $\hat{\beta}_{0}$ & 0,9769 & 0,5689 & 0,3242 & 1,0172 & 0,6183 & 0,3826 \\
\hline & $\hat{\beta}_{1}$ & 1,2403 & 0,2829 & 0,1378 & 0,9873 & 0,3090 & 0,0956 \\
\hline & $\hat{\beta}_{2}$ & 0,9565 & 0,0322 & 0,0029 & 1,0019 & 0,0351 & 0,0012 \\
\hline & $\hat{\sigma}_{e}^{2}$ & 7,5487 & - & 43,1570 & 0,9340 & - & 0,2866 \\
\hline \multirow{4}{*}{1000} & $\hat{\beta}_{0}$ & 1,1045 & 0,5242 & 0,2857 & 1,0049 & 0,5767 & 0,3326 \\
\hline & $\hat{\beta}_{1}$ & 1,1701 & 0,2474 & 0,0902 & 0,9984 & 0,2720 & 0,0740 \\
\hline & $\hat{\beta}_{2}$ & 0,9635 & 0,0268 & 0,0020 & 1,0001 & 0,0293 & 0,0009 \\
\hline & $\hat{\sigma}_{e}^{2}$ & 7,3512 & - & 40,4760 & 0,9679 & - & 0,1457 \\
\hline \multirow{4}{*}{2000} & $\hat{\beta}_{0}$ & 0,9936 & 0,3670 & 0,1347 & 0,9973 & 0,4064 & 0,1651 \\
\hline & $\hat{\beta}_{1}$ & 1,2500 & 0,1760 & 0,0935 & 1,0026 & 0,1950 & 0,0380 \\
\hline & $\hat{\beta}_{2}$ & 0,9529 & 0,0195 & 0,0026 & 0,9996 & 0,0215 & 0,0005 \\
\hline & $\hat{\sigma}_{e}^{2}$ & 7,3875 & - & 40,8670 & 0,9859 & - & 0,0712 \\
\hline \multirow{4}{*}{3000} & $\hat{\beta}_{0}$ & 0,9684 & 0,2987 & 0,0902 & 1,0132 & 0,3317 & 0,1102 \\
\hline & $\hat{\beta}_{1}$ & 1,2741 & 0,1446 & 0,0960 & 0,9932 & 0,1608 & 0,0259 \\
\hline & $\hat{\beta}_{2}$ & 0,9492 & 0,0161 & 0,0028 & 1,0007 & 0,0179 & 0,0003 \\
\hline & $\hat{\sigma}_{e}^{2}$ & 7,3451 & - & 40,3040 & 0,9999 & - & 0,0456 \\
\hline \multirow{4}{*}{4000} & $\hat{\beta}_{0}$ & 1,1363 & 0,2514 & 0,0818 & 0,9989 & 0,2770 & 0,0768 \\
\hline & $\hat{\beta}_{1}$ & 1,1793 & 0,1201 & 0,0466 & 1,0002 & 0,1323 & 0,0175 \\
\hline & $\hat{\beta}_{2}$ & 0,9605 & 0,0132 & 0,0017 & 1,0000 & 0,0146 & 0,0002 \\
\hline & $\hat{\sigma}_{e}^{2}$ & 7,3612 & - & 40,4980 & 0,9903 & - & 0,0351 \\
\hline
\end{tabular}


Como se pode observar na tabela acima, os estimativas para os parâmetros $\beta$ e $\sigma_{e}^{2}$ obtidos pelo método de "escore corrigido"se aproximam dos valores selecionados em nossa simulação. Observa-se também, que as estimativas para os parâmetros $\beta$ obtidos pelo método "naive"se aproximam, mais não quanto as estimativas obtidas pelo método de "escore corrigido", se consideramos $\sigma_{e}^{2}$ obtido pelo método "naive" observa-se que a estimativa esta muito afastado do valor selecionado para a simulação, além disso, observa-se que quanto o tamanho da amostra aumenta o Erro Quadrático Médio (EQM) é mais pequeno no método de "escore corrigido" que no método "naive". Uma representação gráfica é apresentada a continuação,

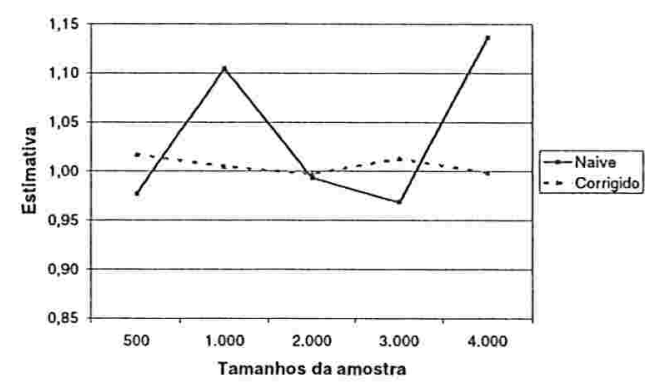

(a) Estimativa para $\beta_{0}$

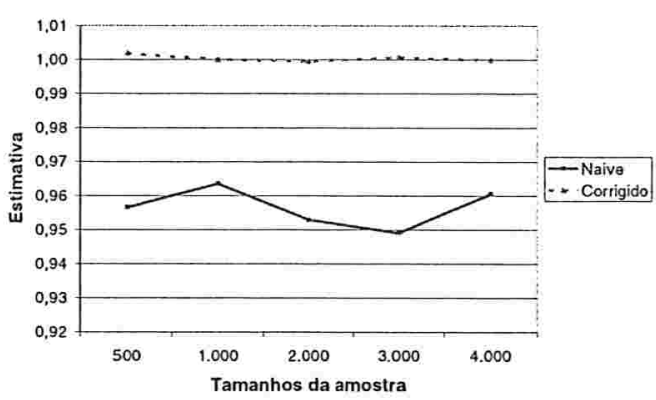

(c) Estimativa para $\beta_{2}$

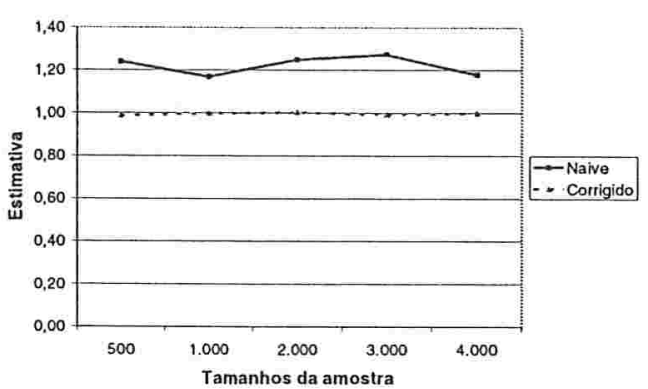

(b) Estimativa para $\beta_{1}$

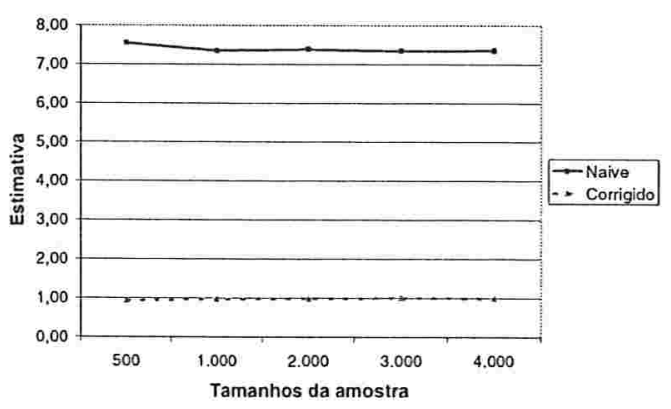

(d) Estimativa para $\sigma_{e}^{2}$

Fig. 2.1. Comportamento das estimativas "naive"e "escore corrigido"segundo o tamanho da amostra 
Nas figuras acima podemos observar que os valores correspondentes aos estimadores dos parâmetros pelo método "naive"não são tão bons como os estimadores obtidos pelo método "escore corrigido" na presente simulação.

\section{b. Um caso prático.}

Para observar melhor as vantagem do método de escore corrigido consideramos o exemplo de Fuller (1987) onde é apresentado uma simulação com 120 observações (pag. 266) para um modelo quadrático. Para a aplicação do método de mínimos quadrados generalizados ele supôs que $\sigma_{u}^{2}=0,09$ para a análise. Os dados encontram-se no anexo deste trabalho, sendo as variáveis consideradas $Y_{1}$ e $W$.

\section{Resultados}

Os dados apresentam a seguinte dispersão

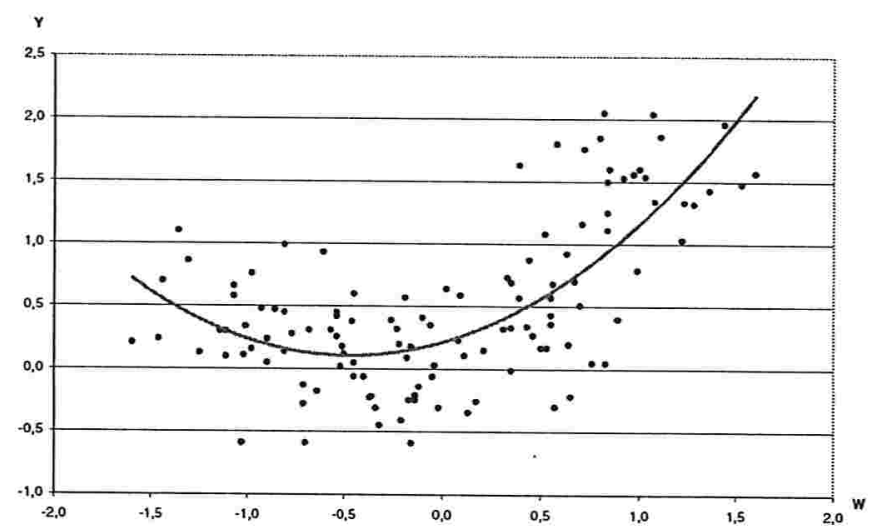

Fig. 2.2. Gráfico da dispersão das variáveis $Y_{1}$ e $W$

Da figura acima observa-se que os dados se alinham de acordo a uma curvatura, segundo a linha vermelha traçado no gráfico poderia tratar-se de uma curvatura quadrática, na construção do modelo consideramos um modelo polinomial de grau dois para analisar a curvatura observada. Os resultados obtidos a partir do método "naive", "escore corrigido"e o apresentado por fuller é descrito na seguinte tabela 
Tabela 2.2. Comparação das estimativas segundo os métodos "naive", "escore corrigido"e de Fuller

\begin{tabular}{|c|c|c||c|c||c|c|}
\hline \multirow{2}{*}{ Parâmetro } & \multicolumn{2}{|c||}{ Naive } & \multicolumn{2}{c||}{ Escore corrigido } & \multicolumn{2}{c|}{ Fuller } \\
\cline { 2 - 7 } & Estimativa & Desv.Pad. & Estimativa & Desv.Pad. & Estimativa & Desv.Pad. \\
\hline$\beta_{0}$ & 0,215 & 0,058 & $-0,024$ & 0,041 & $-0,005$ & 0,108 \\
$\beta_{1}$ & 0,459 & 0,054 & 0,543 & 0,037 & 0,540 & 0,098 \\
$\beta_{2}$ & 0,482 & 0,068 & 1,047 & 0,063 & 1,007 & 0,230 \\
$\sigma_{e}^{2}$ & 0,203 & - & 0,081 & - & 0,090 & - \\
\hline
\end{tabular}

Da tabela acima pode-se observar que tanto o modelo proposto por Fuller (1987) quanto o modelo de "escore corrigido" apresentam estimativas bastante semelhantes, enquanto o método "naive" esta fornecendo estimativas diferentes a os outros dois métodos. Para observar a similitude ou diferencias entre os métodos, consideramos o gráfico a seguir,

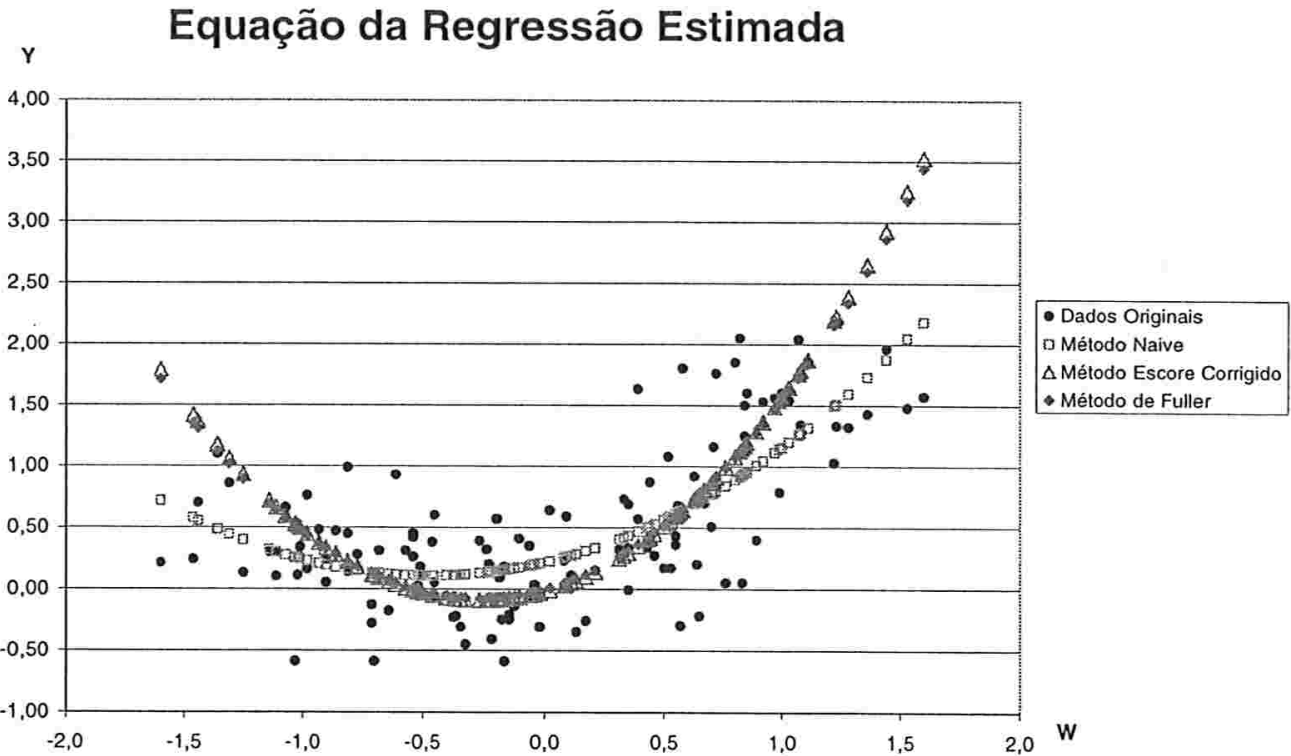

Fig. 2.3. Comportamento das estimativas dos três modelos 
Na figura de acima observa-se as estimativas de Fuller (1987) definido pelos pontos "今" e as estimativas do método de escore corrigido definido pelos pontos “ $\triangle$ " são quase coincidentes, enquanto as estimativas pelo método naive definidos pelos pontos "অ" segue outro comportamento diferente aos outros dois métodos.

Quando consideramos o método escore corrigido e desejamos saber que parâmetro em estudo realmente existe em um modelo quadrático, a tabela a seguir apresenta os resultados considerando os testes de Razão de Verossimilhança (R.V.), Wald e Escore para avaliar a hipótese $H_{0}: \beta_{0}=0$ vs $H_{1}: \beta_{0} \neq 0 ; H_{0}: \beta_{1}=0$ vs $H_{1}: \beta_{1} \neq 0 ; H_{0}: \beta_{2}=0$ vs $H_{1}: \beta_{2} \neq 0$, segundo a metodologia apresentada.

Tabela 2.3. Teste de Hipótese para os parâmetros do modelo.

\begin{tabular}{|c|c|c|c|c|c|c|c|}
\hline Parâmetro & Estimador & R.V. & p-value & Wald & p-value & Escore & p-value \\
\hline$\beta_{0}$ & $-0,024409$ & 1,7627 & 0,18428 & 0,0458 & 0,83037 & 17,0670 & 0,00003 \\
$\beta_{1}$ & 0,543150 & 219,47 & 0,00000 & 29,132 & 0,00000 & 1653,40 & 0,00000 \\
$\beta_{2}$ & 1,046900 & 681,94 & 0,00000 & 18,672 & 0,00001 & 24906,0 & 0,00000 \\
\hline
\end{tabular}

Notemos que ao efetuar o teste no exemplo proposto por Fuller (1987) para cada um dos parâmetros envolvidos em um modelo quadrático, os testes da razão de verossimilhanças, wald e escore consideram a presencia dos parâmetros $\beta_{1}$ e $\beta_{2}$ no modelo, enquanto só o teste de escore aceita a presencia do parâmetro $\beta_{0}$ no modelo. Como $\beta_{0}$ não vai modificar muito nossas predições, podemos definir que o modelo a considerar para esse conjunto de dados seria

$$
Y_{i}=\underset{(0,041)}{-0,024} \underset{(0,037)}{0,543} W_{i}+\underset{(0,063)}{1,047} W_{i}^{2}+e_{i}
$$

Uma representação gráfica dos modelos linear e quadrático para o método escore corrigido é apresentado a continuação, 


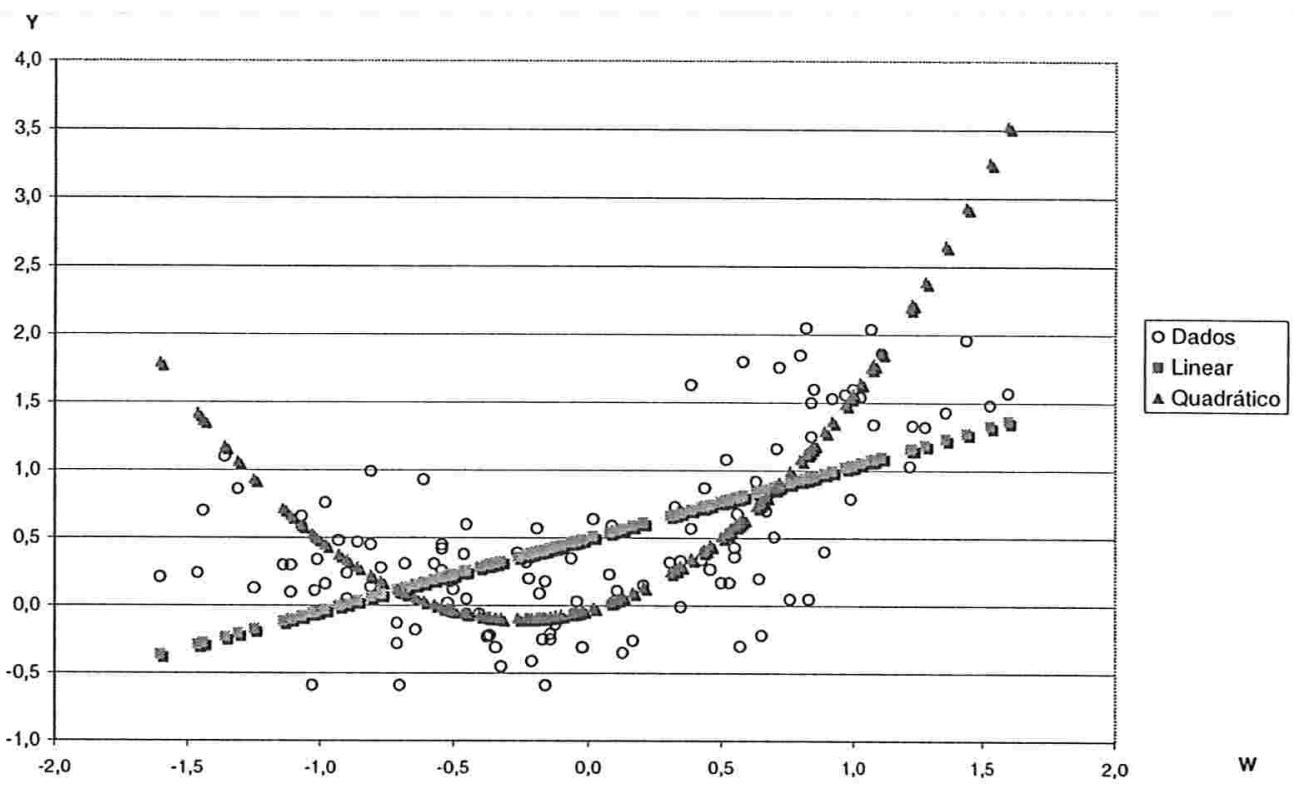

Fig. 2.4. Comportamento das estimativas do modelo linear e quadrático no escore corrigido

Do gráfico acima podemos dizer que o modelo quadrático acompanha melhor ao conjunto de dados em estudo. 
2.3 Caso 2: $\lambda=\frac{\sigma_{e}^{2}}{\sigma_{u}^{2}} \quad$ conhecida

\subsubsection{Estimação dos parâmetros}

Nesta seção estudaremos a função escore corrigida derivada segundo a proposta de Bolfarine e Gimenez (1997), a metodologia a apresentar é válida para o modelo funcional quanto ao modelo estrutural.

a) Obtenção da função escore a partir do logaritmo da função verossimilhança "naive"

Dado que $\sigma_{e}^{2}=\lambda \sigma_{u}^{2}$, o logaritmo da função verossimilhança "naive" é definido similarmente à expressão (2.3), isto é,

$$
\ell\left(\beta, \sigma_{u}^{2} ; \mathrm{Y}, \mathrm{W}\right)=-\frac{n}{2} \log (2 \pi \lambda)-\frac{n}{2} \log \left(\sigma_{u}^{2}\right)-\frac{1}{2 \lambda \sigma_{u}^{2}} \sum_{i=1}^{n}\left(Y_{i}-\sum_{j=0}^{p} \beta_{j} W_{i}^{j}\right)^{2}
$$

Considerando os passos vistos na seção 2.2.1 (a), temos que o logaritmo da função verossimilhança corrigida é análogo com a expressão (2.9), isto é,

$$
\begin{aligned}
\ell^{*}\left(\beta, \sigma_{u}^{2} ; \mathbf{Y}, \mathbf{W}\right)= & -\frac{n}{2} \log (2 \pi \lambda)-\frac{n}{2} \log \left(\sigma_{u}^{2}\right)-\frac{1}{2 \lambda \sigma_{u}^{2}}\left\{\sum _ { i = 1 } ^ { n } \left[Y_{i}^{2}+\sum_{j=0}^{p} \beta_{j}^{2} t_{i,(2 j)}\right.\right. \\
& \left.\left.-2 Y_{i} \sum_{j=0}^{p} \beta_{j} t_{i,(j)}+2 \sum_{\substack{j=0 \\
s<j}}^{p} \sum_{s=0}^{p} \beta_{j} \beta_{s} t_{i,(j+s)}\right]\right\}
\end{aligned}
$$

onde $t_{i,(j)}$ é dado em (2.27).

Para obter a função escore corrigido, deve-se derivar (2.44) em relação a cada parâmetro, obtendo-se as seguintes expressões:

$$
\mathrm{U}^{*}\left(\beta_{s}\right)=\frac{1}{\lambda \sigma_{u}^{2}} \sum_{i=1}^{n}\left(Y_{i} t_{i,(s)}-\sum_{j=0}^{p} \beta_{j} t_{i,(j+s)}\right),
$$

para $s=0,1, \ldots, p$ e 


$$
\begin{aligned}
\mathrm{U}^{*}\left(\sigma_{u}^{2}\right)= & -\frac{n}{2 \sigma_{u}^{2}}+\frac{1}{2 \lambda \sigma_{u}^{4}} \sum_{i=1}^{n}\left[Y_{i}^{2}+\sum_{j=0}^{p} \beta_{j}^{2} t_{i,(2 j)}-2 Y_{i} \sum_{j=0}^{p} \beta_{j} t_{i,(j)}+2 \sum_{\substack{j=0 \\
j<s}}^{p} \sum_{s=0}^{p} \beta_{j} \beta_{s} t_{i,(j+s)}\right] \\
& \left.-\frac{1}{2 \lambda \sigma_{u}^{2}} \sum_{i=1}^{n}\left[\sum_{j=0}^{p} \beta_{j}^{2} \frac{\partial t_{i,(2 j)}}{\partial \sigma_{u}^{2}}-2 Y_{i} \sum_{j=0}^{p} \beta_{j} \frac{\partial t_{i,(j)}}{\partial \sigma_{u}^{2}}+2 \sum_{j=0}^{p} \sum_{\substack{j=0 \\
j<s}}^{p} \beta_{j} \beta_{s} \frac{\partial t_{i,(j+s)}}{\partial \sigma_{u}^{2}}\right]\right\}(2.46)
\end{aligned}
$$

O calculo das derivadas $\frac{\partial}{\partial \sigma_{u}^{2}} t_{i,(j)}$ tem a forma seguinte,

$$
\frac{\partial}{\partial \sigma_{u}^{2}} t_{i,(j+1)}=W_{i} \frac{\partial}{\partial \sigma_{u}^{2}} t_{i,(j)}-j\left(t_{i,(j-1)}+\sigma_{u}^{2} \frac{\partial}{\partial \sigma_{u}^{2}} t_{i,(j-1)}\right)
$$

b) Obtenção da função escore a partir da função escore "naive".

Considerando o logaritmo da função verossimilhança "naive" apresentada na expressão (2.43) e derivando esta em relação a cada parâmetro temos as seguintes funções escore "naive":

$$
\begin{gathered}
\mathrm{U}\left(\beta_{s}\right)=\frac{1}{\lambda \sigma_{u}^{2}} \sum_{i=1}^{n}\left[Y_{i} W_{i}^{s}-\sum_{j=0}^{p} \beta_{j} W_{i}^{j+s}\right], \\
\mathrm{U}\left(\sigma_{u}^{2}\right)=-\frac{1}{2 \sigma_{u}^{2}}+\frac{1}{2 \lambda \sigma_{u}^{4}} \sum_{i=1}^{n}\left(Y_{i}-\sum_{j=0}^{p} \beta_{j} W_{i}^{j}\right)^{2} .
\end{gathered}
$$

Repetindo os passos vistos na seção 2.2.1 (b), temos que a função escore corrigida é,

$$
\begin{aligned}
\mathrm{U}^{*}\left(\beta_{s}\right)= & \frac{1}{\lambda \sigma_{u}^{2}} \sum_{i=1}^{n}\left[Y_{i} t_{i,(s)}-\sum_{j=0}^{p} \beta_{j} t_{i,(j+s)}\right], \quad s=1, \ldots, p, \\
\mathrm{U}^{*}\left(\sigma_{u}^{2}\right)= & -\frac{n}{2 \sigma_{u}^{2}}+\frac{1}{2 \lambda \sigma_{u}^{4}} \sum_{i=1}^{n}\left(Y_{i}^{2}+\sum_{j=0}^{p} \beta_{j}^{2} t_{i,(2 j)}-2 Y_{i} \sum_{j=0}^{p} \beta_{j} t_{i,(j)}\right. \\
& \left.+2 \sum_{j=0}^{p} \sum_{s=0}^{p} \beta_{j} \beta_{s} t_{i,(j+s)}\right) .
\end{aligned}
$$


Sendo $t_{i,(m)}$ uma variável que esta em função de $\sigma_{u}^{2}$, e que tem a forma como na expressão (2.27).

\section{c) Observações}

- A condição fundamental na proposta de Nakamura, estabelecendo que $E(\bullet \mid Y, X)$ e $\frac{\partial(\bullet)}{\partial \theta}$ serem permutáveis, não é satisfeita neste caso, pois observemos que as expressões (2.46) e (2.50) não coincidem. Para este caso adotamos a metodologia proposta por Bolfarine e Gimenez (1997).

- A metodologia proposta por Bolfarine e Gimenez (1997) aplicada aos modelos polinomiais não foi explorada até agora.

- No caso do modelo com $\frac{\sigma_{e}^{2}}{\sigma_{u}^{2}}=\lambda$ conhecido não dá para usar a verossimilhança corrigida mas dá para usar o escore corrigido. Bolfarine e Gimenez (1997), descobriram esta mesma situação num modelo lineảr, agora segundo nosso análise este problema pode se extender também para o caso do modelo polinomial.

- De acordo com a metodo da função escore corrigido proposta por Bolfarine e Gimenez (1997), temos que as equações lineares definidas a partir das expressões (2.49) e (2.50) permitem-nos obter um processo iterativo para a estimação dos parâmetros. Inicialmente igualamos (2.49) a o resultado em

$$
\left(\begin{array}{cccc}
n & \sum_{i=1}^{n} t_{i,(1)} & \cdots & \sum_{i=1}^{n} t_{i,(p)} \\
\sum_{i=1}^{n} t_{i,(1)} & \sum_{i=1}^{n} t_{i,(2)} & \cdots & \sum_{i=1}^{n} t_{i,(p+1)} \\
\vdots & \vdots & \cdots & \vdots \\
\sum_{i=1}^{n} t_{i,(p)} & \sum_{i=1}^{n} t_{i,(p+1)} & \cdots & \sum_{i=1}^{n} t_{i,(2 p)}
\end{array}\right)\left(\begin{array}{c}
\hat{\beta}_{0} \\
\hat{\beta}_{1} \\
\vdots \\
\hat{\beta}_{p}
\end{array}\right)=\left(\begin{array}{c}
\sum_{i=1}^{n} Y_{i} \\
\sum_{i=1}^{n} Y_{i} t_{i,(1)} \\
\vdots \\
\sum_{i=1}^{n} Y_{i} t_{i,(p)}
\end{array}\right),
$$

onde cada $t_{i,(s)}$ é obtido segundo a expressão (2.27), enquanto que o estimador de $\sigma_{u}^{2}$ segue da expressão 


$$
\begin{aligned}
\hat{\sigma}_{u}^{2}= & \frac{1}{\lambda n} \sum_{i=1}^{n}\left[Y_{i}^{2}+\sum_{j=0}^{p} \hat{\beta}_{j}^{2} t_{i,(2 j)}-2 Y_{i} \sum_{j=0}^{p} \hat{\beta}_{j} t_{i,(j)}\right. \\
& \left.+2 \sum_{\substack{j=0 \\
s<j}}^{p} \sum_{\substack{s=0 \\
s}}^{p} \hat{\beta}_{j} \hat{\beta}_{s} t_{i,(j+s)}\right] .
\end{aligned}
$$

- Devido à inter dependência das expressões (2.51) e (??), desenvolvemos um processo iterativo tipo Newton-Rapshon modificado para acelerar o processo de convergência para estimar o parâmetro $\sigma_{u}^{2}$. Para isto consideramos a função escore corrigida para $\sigma_{u}^{2}$ que se obtém a partir da expressão (2.50). A derivada desta com relação a $\sigma_{u}^{2}$ é a informação observada deste parâmetro. O algoritmo é composto dos seguintes passos:

1. Escolha-se um valor inicial para $\sigma_{u}^{2}$.

2. Substituir $\sigma_{u}^{2}$ na expressão (2.51).

3. Procede-se com o método de Newton-Raphson modificado para obter o novo valor de $\sigma_{u}^{2}$, isto é,

$$
\sigma_{u}^{2(m+1)}=\sigma_{u}^{2(m)}+\mathbf{I}^{-1}\left(\sigma_{u}^{2(m)}\right) \mathbf{U}\left(\sigma_{u}^{2(m)}\right), \quad m=0,1, \ldots
$$

$\mathrm{I}\left(\sigma_{u}^{2(m)}\right)$ é obtida derivando a expressão (2.50) com relação a $\sigma_{u}^{2}$, e $\mathrm{U}\left(\sigma_{u}^{2(m)}\right)$ é obtida da expressão (2.50).

4. Retornar ao passo 2. 
d) Exemplos.

\section{Polinômio de primeiro grau}

O estimador de $\beta_{0}$ é dado pela expressão (2.30). Em (2.32) fazemos $\hat{\sigma}_{\boldsymbol{e}}^{2}=\lambda \hat{\sigma}_{u}^{2}$ obtendo

$$
\hat{\sigma}_{u}^{2}=\frac{S_{Y Y}+\hat{\beta}_{1}^{2} S_{W W}-2 \hat{\beta}_{1} S_{Y W}}{\lambda+\hat{\beta}_{1}^{2}} .
$$

Ao substituir (2.54) em (2.31), obtemos

$$
\hat{\beta}_{1}=\frac{-\left(\lambda S_{W W}-S_{Y Y}\right)+\sqrt{\left(\lambda S_{W W}-S_{Y Y}\right)^{2}+4 \lambda S_{Y W}}}{2 S_{Y W}} .
$$

Se $\lambda=1$ temos

$$
\hat{\beta}_{1}=\frac{\left(S_{Y Y}-S_{W W}\right)+\sqrt{\left(S_{Y Y}-S_{W W}\right)^{2}+4 S_{Y W}}}{2 S_{Y W}},
$$

que é o estimador obtido por Bolfarine e Gimenez (1997).

\section{Polinômio de segundo grau}

Para estimar os parâmetros deste modelo procedemos do seguinte modo:

(a) Atribuir um valor inicial a $\sigma_{u}^{2}$.

(b) De (2.49) formamos o sistema:

$$
\left(\begin{array}{ccc}
n & \sum_{i=1}^{n} t_{i,(1)} & \sum_{i=1}^{n} t_{i,(2)} \\
\sum_{i=1}^{n} t_{i,(1)} & \sum_{i=1}^{n} t_{i,(2)} & \sum_{i=1}^{n} t_{i,(3)} \\
\sum_{i=1}^{n} t_{i,(2)} & \sum_{i=1}^{n} t_{i,(3)} & \sum_{i=1}^{n} t_{i,(4)}
\end{array}\right)\left(\begin{array}{l}
\hat{\beta}_{0} \\
\hat{\beta}_{1} \\
\hat{\beta}_{2}
\end{array}\right)=\left(\begin{array}{c}
\sum_{i=1}^{n} Y_{i} \\
\sum_{i=1}^{n} Y_{i} t_{i,(1)} \\
\sum_{i=1}^{n} Y_{i} t_{i,(2)}
\end{array}\right)
$$

onde para os elementos da matriz de acima deve-se considerar, $t_{i,(1)}=W_{i}$, $t_{i,(2)}=W_{i}^{2}-\sigma_{u}^{2}, t_{i,(3)}=W_{i}^{3}-3 W_{i} \sigma_{u}^{2}$ e $t_{i,(4)}=W_{i}^{4}-6 W_{i}^{2} \sigma_{u}^{2}+3 \sigma_{u}^{4}$.

De acordo com (2.50) a função escore corrigido para $\sigma_{u}^{2}$ é dada por 


$$
\begin{aligned}
\mathrm{U}^{*}\left(\sigma_{u}^{2}\right)= & -\frac{n}{2 \sigma_{u}^{2}}+\frac{1}{2 \lambda \sigma_{u}^{4}} \sum_{i=1}^{n}\left[Y_{i}^{2}+\sum_{j=0}^{2} \hat{\beta}_{j}^{2} t_{i,(2 j)}-2 Y_{i} \sum_{j=0}^{2} \hat{\beta}_{j} t_{i,(j)}+\right. \\
& \left.+2 \sum_{\substack{j=0 \\
s<j}}^{2} \sum_{s=0}^{2} \hat{\beta}_{j} \hat{\beta}_{s} t_{i,(j+s)}\right],
\end{aligned}
$$

e a informação observada tem a expressão

$$
\mathrm{I}^{*}\left(\sigma_{u}^{2}\right)=\frac{1}{2 \lambda \sigma_{u}^{4}} \sum_{i=1}^{n}\left[n \lambda+\beta_{1}^{2}+6 \beta_{2}^{2} t_{i,(2)}-2 Y_{i} \beta_{2}+2 \beta_{0} \beta_{2}+6 \beta_{1} \beta_{2} t_{i,(1)}\right],(2.5
$$

(c) Obter o novo valor de $\sigma_{u}^{2}$ a partir do algoritmo Newton-Raphson, como na expressão (2.53)

\subsubsection{Obtenção da matriz de covariâncias assintóticas}

Para a obtenção da matriz de covariâncias assintóticas consideramos a expressão (1.21)

$$
£^{*}=\frac{1}{n}\left(\mathbf{I}^{*}(\hat{\theta} ; \mathbf{W}, \mathbf{Y})\right)^{-1} \mathbf{S}^{*}(\hat{\theta} ; \mathbf{W}, \mathbf{Y})\left(\mathbf{I}^{*}(\hat{\theta} ; \mathbf{W}, \mathbf{Y})\right)^{-1},
$$

onde,

$$
\mathbf{I}^{*}(\hat{\beta} ; \mathbf{W}, \mathbf{Y})=-\frac{1}{n} \sum_{i=1}^{n} \frac{\partial}{\partial \beta} U_{i}^{*}(\hat{\beta} ; \mathbf{W}, \mathbf{Y})
$$

e

$$
\mathbf{S}^{*}(\hat{\beta} ; \mathbf{W}, \mathbf{Y})=\frac{1}{n} \sum_{i=1}^{n} \mathbf{U}_{i}^{*}(\hat{\beta} ; \mathbf{W}, \mathbf{Y}) \mathbf{U}_{i}^{*}(\hat{\beta} ; \mathbf{W}, \mathbf{Y})^{t}
$$

concluindo que

$$
\sqrt{n}(\hat{\beta}-\beta) \stackrel{D}{\longrightarrow} N_{p+1}\left(0, £^{*}\right)
$$

\subsubsection{Teste de Hipótese.}

Devido à falta de uma da função verossimilhança corrigida adequada para este caso em estudo, recorremos à função escore corrigido, que nos permite propor as seguintes estatísticas de teste: 
- Teste de wald.

Esta é definido pela expressão seguinte

$$
Q_{W}=\left(\hat{\beta}-\beta^{(0)}\right)^{t} £(\beta)\left(\hat{\beta}-\beta^{(0)}\right) \sim \chi_{(d)}^{2}
$$

onde $d=\operatorname{dim}\left(H_{01}\right)-\operatorname{dim}\left(H_{0}\right)=(p+1)-(p+1-q)=q$, sendo as hipóteses de interesse são $H_{0}: \beta=\beta^{(0)}$ e $H_{1}: \beta \neq \beta^{(0)}$ e q representa o número de parâmetros a serem testados.

- Teste do escore (Rao)

Esta é definido pela expressão seguinte

$$
Q_{E}=U^{*}\left(\beta^{(0)}\right) £\left(\beta^{(0)}\right)^{-1} U^{*}\left(\beta^{(0)}\right) \sim \chi_{(d)}^{2}
$$

\subsubsection{Aplicações numéricas}

Para avaliar a metodologia apresentada consideramos duas situações, uma simulação de Monte Carlo e uma situação prática.

a. Um estudo de simulação

Para esta simulação consideramos os seguintes processos

- Para $n$ especificado selecionamos $X_{i} \sim \operatorname{Poisson}(n, 1)$ independente, $u_{i} \sim N(0 ; 0,05)$ independente e $e_{i} \sim N(0 ; 1)$ independente, para $i=1,2, \ldots, n$.

- Obter os dados fazendo,

$$
\begin{gathered}
W_{i}=X_{i}+u_{i} \\
Y_{i}=\beta_{0}+\beta_{1} X_{i}+\beta_{2} X_{i}^{2}+e_{i} \quad i=1,2, \ldots, n,
\end{gathered}
$$

sendo $\beta_{0}, \beta_{1}$ e $\beta_{2}$ iguais a um.

- Para efeitos práticos deve-se considerar as expressões (2.57)-(2.59), para a estimação dos parâmetros.

\section{Resultados}

Em seguida os resultados obtidos da simulação implementada acima em linguagem OX (Doornik, 1998) são apresentados na tabela seguinte 
Tabela 2.4. Estimativas segundo os métodos "naive"e "escore corrigido" para diferentes tamanhos de amostra.

\begin{tabular}{|c|c|c|c|c|c|c|c|}
\hline \multirow[t]{2}{*}{$\mathrm{n}$} & \multirow[t]{2}{*}{ Parâmetro } & \multicolumn{3}{|c|}{ Naive } & \multicolumn{3}{|c|}{ Escore corrigido } \\
\hline & & Estimativa & Desv.Pad. & EQM & Estimativa & Desv.Pad. & EQM \\
\hline \multirow{4}{*}{500} & $\hat{\beta}_{0}$ & 1,25450 & 0,07231 & 0,07002 & 1,00950 & 0,13310 & 0,01781 \\
\hline & $\hat{\beta}_{1}$ & 0,75959 & 0,15865 & 0,08297 & 0,93902 & 0,37569 & 0,14486 \\
\hline & $\hat{\beta}_{2}$ & 0,88381 & 0,07027 & 0,01844 & 1,02640 & 0,14208 & 0,02088 \\
\hline & $\hat{\sigma}_{u}^{2}$ & 3,85310 & - & 13,10600 & 0,24587 & - & 0,00053 \\
\hline \multirow{4}{*}{1000} & $\hat{\beta}_{0}$ & 1,29960 & 0,05332 & 0,09258 & 1,00590 & 0,09813 & 0,00967 \\
\hline & $\hat{\beta}_{1}$ & 0,77550 & 0,11952 & 0,06468 & 0,96082 & 0,27916 & 0,07947 \\
\hline & $\hat{\beta}_{2}$ & 0,86672 & 0,05261 & 0,02053 & 1,01780 & 0,10761 & 0,01190 \\
\hline & $\hat{\sigma}_{u}^{2}$ & 3,95360 & - & 13,77800 & 0,24770 & - & 0,00026 \\
\hline \multirow{4}{*}{2000} & $\hat{\beta}_{0}$ & 1,24150 & 0,03475 & 0,05952 & 1,00430 & 0,06062 & 0,00369 \\
\hline & $\hat{\beta}_{1}$ & 0,86194 & 0,07910 & 0,02532 & 0,98314 & 0,20693 & 0,04310 \\
\hline & $\hat{\beta}_{2}$ & 0,83327 & 0,03684 & 0,02916 & 1,00800 & 0,08313 & 0,00697 \\
\hline & $\hat{\sigma}_{u}^{2}$ & 3,69840 & - & 11,91800 & 0,24931 & - & 0,00013 \\
\hline \multirow{4}{*}{3000} & $\hat{\beta}_{0}$ & 1,26530 & 0,03077 & 0,07135 & 1,00230 & 0,05233 & 0,00274 \\
\hline & $\hat{\beta}_{1}$ & 0,83593 & 0,06779 & 0,03151 & 0,98985 & 0,17006 & 0,02902 \\
\hline & $\hat{\beta}_{2}$ & 0,83954 & 0,03151 & 0,02674 & 1,00340 & 0,06838 & 0,00469 \\
\hline & $\hat{\sigma}_{u}^{2}$ & 3,78680 & - & 12,52900 & 0,24996 & - & 0,00009 \\
\hline \multirow{4}{*}{4000} & $\hat{\beta}_{0}$ & 1,26600 & 0,02577 & 0,07142 & 0,99900 & 0,04587 & 0,00210 \\
\hline & $\hat{\beta}_{1}$ & 0,86994 & 0,05854 & 0,02034 & 0,99886 & 0,14884 & 0,02215 \\
\hline & $\hat{\beta}_{2}$ & 0,82695 & 0,02662 & 0,03066 & 1,00160 & 0,05926 & 0,00351 \\
\hline & $\hat{\sigma}_{u}^{2}$ & 3,79670 & - & 12,59200 & 0,24953 & - & 0,00006 \\
\hline
\end{tabular}


Como se pode observar na tabela acima, os estimadores dos parâmetros $\beta$ obtidos pelo método do escore corrigido atingem aos parâmetros propostos em nossa simulação, da mesma maneira os valores de $\sigma_{u}^{2}$ esta próximos dos valores fixados na simulação, enquanto as estimativas obtidas pelo método "naive" não se aproximam dos verdadeiros valores.

Uma representação gráfica é apresentada a seguir

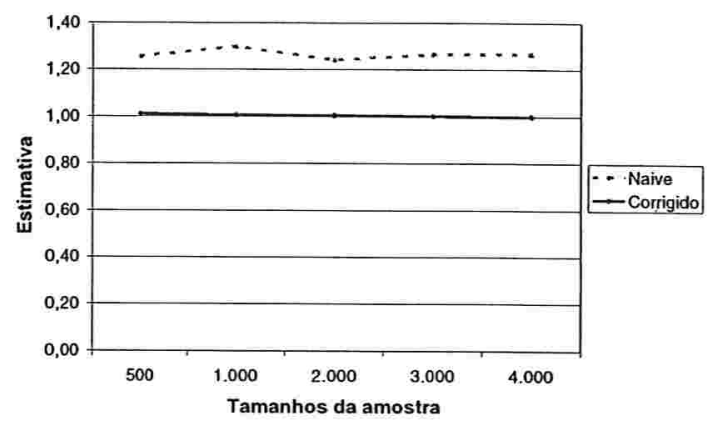

(a) Estimativa para $\beta_{0}$

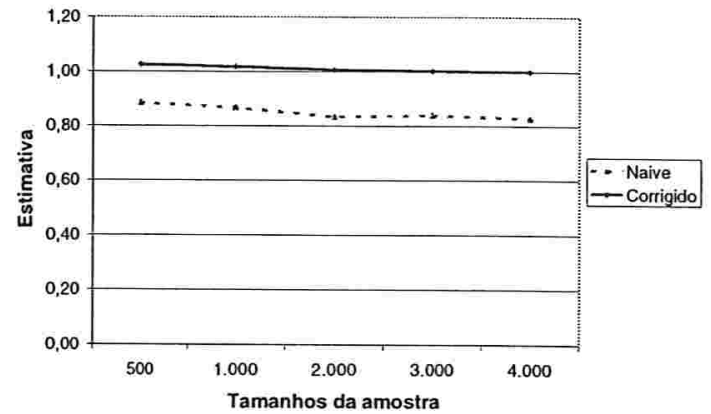

(c) Estimativa para $\beta_{2}$

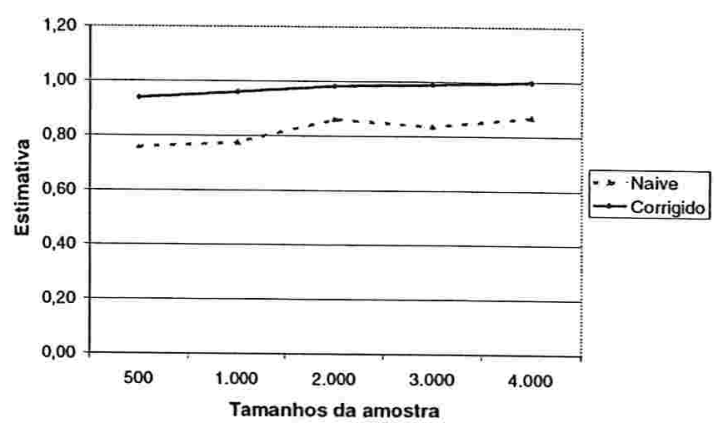

(b) Estimativa para $\beta_{1}$

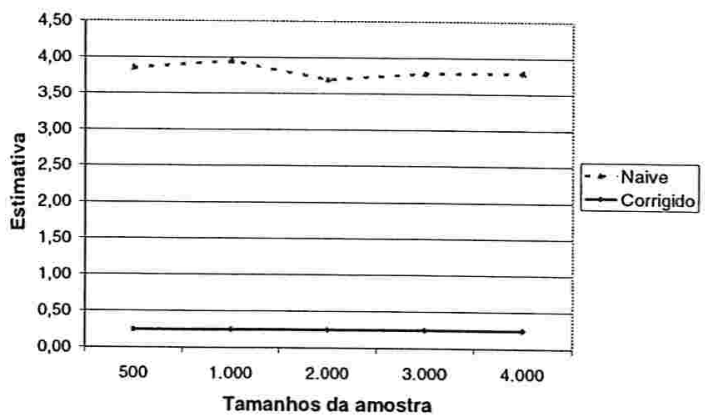

(d) Estimativa para $\sigma_{e}^{2}$

Fig. 2.5. Comportamento das estimativas "naive"e "escore corrigido"segundo o tamanho da amostra 
$\mathrm{Na}$ figura de acima podemos observar que os valores correspondentes aos estimativas para os parâmetros "naive" não conseguem atingir os valores implementados na presente simulação, enquanto as estimativas para os parâmetros "escore corrigido"se aproximam adequadamente.

\section{b. Um caso prático.}

Neste caso consideramos um outro exemplo proposto por Fuller (1987) com 120 observações (pag. 248), adotando para isto um modelo quadrático, em que $\sigma_{u}^{2}=0,09$ e $\lambda=1$ para a análise, Os dados encontram-se no anexo (tabela 4.25) sendo as variáveis consideradas $Y_{2}$ e $W$.

\section{Resultados}

Os dados considerados apresentam a seguinte dispersão

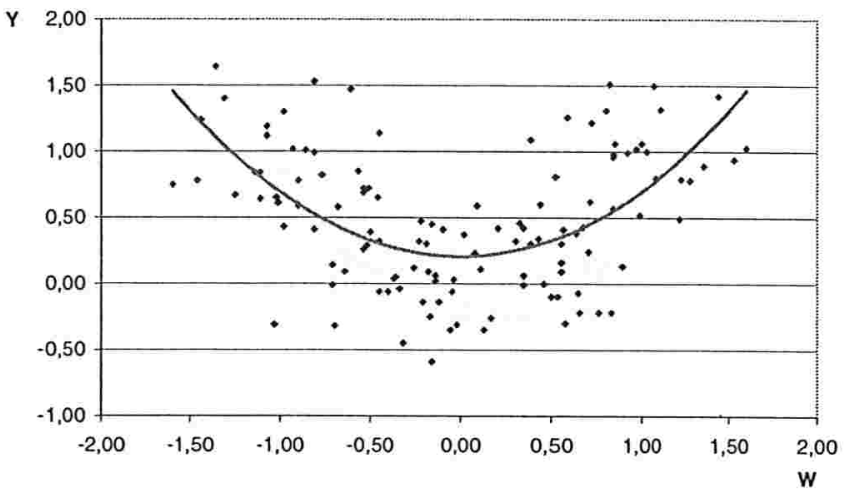

Fig. 2.6. Gráfico da dispersão das variáveis $Y_{2}$ e $W$ 
Tabela 2.5. Estimativas segundo as metodologias "naive" e "escore corrigido".

\begin{tabular}{|c|c|c||c|c|}
\hline \multirow{2}{*}{ Parâmetro } & \multicolumn{2}{|c||}{ Naive } & \multicolumn{2}{c|}{ Escore Corrigido } \\
\cline { 2 - 5 } & Estimativa & Desvio Padrão & Estimativa & Desvio Padrão \\
\hline$\beta_{0}$ & 0,20469 & 0,054678 & $-0,023631$ & 0,10310 \\
$\beta_{1}$ & 0,00178 & 0,050845 & 0,004183 & 0,09509 \\
$\beta_{2}$ & 0,48967 & 0,064726 & 1,027800 & 0,22073 \\
$\sigma_{u}^{2}$ & 0,18328 & - & 0,087207 & - \\
\hline
\end{tabular}

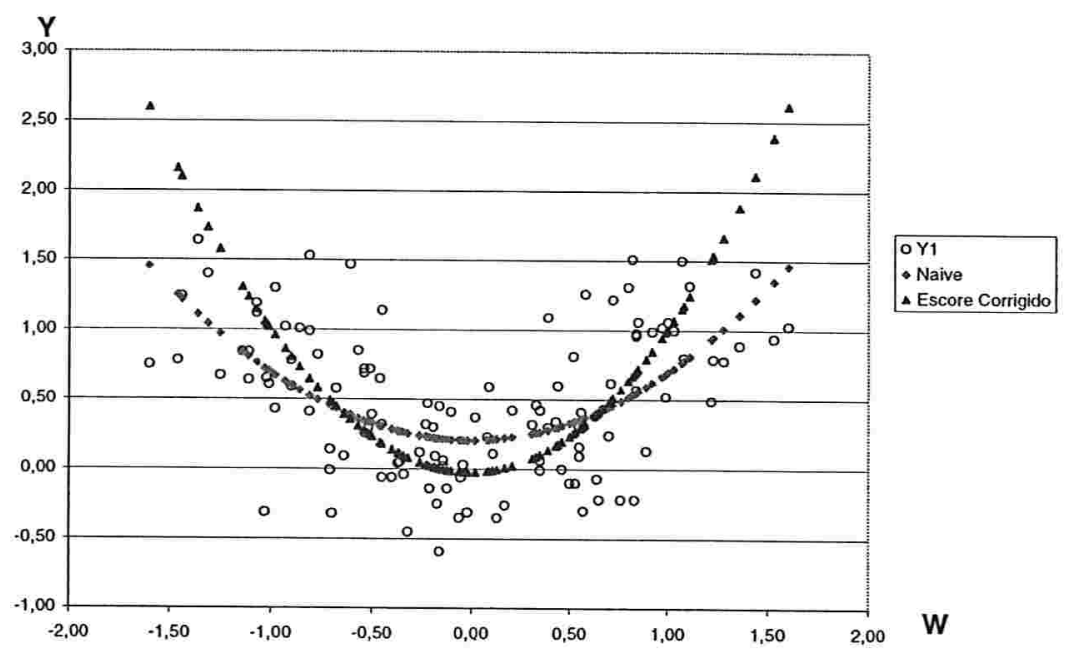

Fig. 2.7. Gráfico do comportamento das estimativas dos métodos "naive"e "escore corrigido"

Podemos observar da tabela (2.5) e da figura (2.7) que o modelo quadrático quando é usado o método do escore corrigido se ajusta melhor aos dados que o método naive, por outro lado na tabela (2.5) observa-se que, quem estima melhor o valor de $\sigma_{u}^{2}$ é o método do escore corrigido. 
Tabela 2.6. Avaliação das estimativas do método "escore corrigido", usando o teste de wald.

\begin{tabular}{|c|c|c|c|}
\hline Parâmetro & Estimativa & Teste de wald & nível descritivo \\
\hline$\beta_{0}$ & $-0,023631$ & 0,00071228 & 0,97871 \\
$\beta_{1}$ & 0,004183 & 0,00001898 & 0,99652 \\
$\beta_{2}$ & 1,027800 & 6,17680000 & 0,01294 \\
\hline
\end{tabular}

Segundo o teste de wald podemos observar que o modelo só considera a $\beta_{2}$ como o único parâmetro válido para o modelo, a partir disto reformulamos o modelo sendo este o seguinte

$$
Y_{i}=\underset{(0,10307)}{-0,023622}+\underset{(0,22067)}{1,0278} W_{i}^{2}
$$

Fuller (1987) utilizou o mesmo conjunto de dados para avaliar as estimativas segundo o método de momentos e com o programa SUPER CARP obteve as estimativas seguintes,

$$
Y_{i}=\underset{(0,074)}{0,016}+\underset{(0,134)}{1,019} W_{i}^{2}
$$

se bem é certo ele não considerou em seu análise a relação $\lambda=\frac{\sigma_{e}^{2}}{\sigma_{u}^{2}}$ conhecida, mas o interessante é que com uma metodologia diferente nos obtivemos resultados próximos.

\subsection{Influência Local}

\subsubsection{Metodologia}

Um dos métodos mais modernos de diagnóstico foi proposto por Cook (1986). A idéia básica consiste em estudar o comportamento de algumas medidas particulares de influência segundo pequenas perturbações nos dados modelo.

Em nosso estudo analisaremos só o caso 1 (onde $\sigma_{u}^{2}$ é conhecida), já que no caso 2 ( $\lambda$ conhecida), não dispomos do logaritmo da função verossimilhança corrigida. 
A influência das perturbações sobre os estimadores de máxima verossimilhança corrigida de $\theta$ pode ser quantificado pelo afastamento da verossimilhança, definido pela expressão

$$
F(v)=2\left[\ell^{*}(\hat{\beta})-\ell^{*}(\hat{\beta}(v))\right]
$$

onde $F(v)$ é o distanciamento entre os logaritmos das funções verossimilhança corrigidas, $\ell^{*}(\hat{\theta})$ e $\ell^{*}(\hat{\theta}(v))$ são os logaritmos das funções verossimilhança corrigidas dos modelos postulado e perturbado, respectivamente $\hat{\theta}$ e $\hat{\theta}(v)$ correspondem aos estimadores de máxima verossimilhança corrigida de $\beta, v$ é o vetor de perturbações, de dimensão $n \times 1$.

Cook (1986), com a finalidade de quantificar o distanciamento $F(v)$ avalia a curvatura normal na direção unitária $d$. Para tanto $F(v)$ deve ser uma função não negativa com mínimo global em $v_{0}$, de maneira que a curvatura normal a $F\left(v_{0}\right)$ na direção unitária $d$ é expressa como visto no capitulo 1, isto é,

$$
C(d)=2\left|d^{t} \Delta^{t} f^{-1} \Delta d\right|
$$

onde

$$
\Delta=\frac{\partial^{2} \ell^{*}(\beta \mid v)}{\partial \beta \partial v^{t}}
$$

e

$$
f=-\frac{\partial^{2} \ell^{*}(\beta)}{\partial \beta \partial \beta^{t}}
$$

existem muitas formas de obter a influência local, mas aquela que corresponde a maior curvatura $C_{m a x}$ é a mais usada, esta curvatura produz a maior mudança local nas estimativas dos parâmetros, e esta corresponde ao maior autovalor de $\Delta^{t} f^{-1} \Delta$, sendo o maior distanciamento definido por $d_{\max }$, onde $d_{\max }$ é definida como o autovetor associada ao maior autovalor de $\Delta^{t} f^{-1} \Delta$.

$$
\left.\left(\Delta^{t} f^{-1} \Delta\right)\right|_{v=v_{0} ; \beta=\hat{\beta}^{0}}
$$

onde $\Delta$ é a matriz $(p \times q)$ com elementos da forma,

$$
\Delta_{s j}=\frac{\partial^{2}}{\partial v_{s} \partial \beta_{j}} \ell^{*}(\beta, v ; \mathbf{W}, \mathbf{Y}),
$$

para $j=0,1,2, \ldots, p$, e $s=0,1,2, \ldots, p$, onde $j$ representa os índices dos parâmetros a serem estimados, e $s$ representa o índice correspondente as perturbações a efetuar, $f$ é a matriz de Informação de Fisher observada no modelo postulado $\left(v=v_{0}\right)$, calculada em $\theta=\hat{\theta}$. 


\subsubsection{Esquemas de perturbação}

\section{a) Perturbação da Ponderação dos casos.}

Consideremos o vetor $v$ de dimensão $(n \times 1)$ de pesos aplicados ao logaritmo da função verossimilhança corrigida, onde $v_{i}=1$, significa que a observação é incluída e $v_{i}=0$ significa exclusão para $i=1,2, \ldots, n$.

O logaritmo da função verossimilhança corrigida perturbada tem expressão,

$$
\ell^{*}(\beta, v ; \mathbf{W}, \mathbf{Y})=\sum_{i=1}^{n} v_{i} \ell^{*}\left(\beta, \sigma_{e}^{2} ; W_{i}, Y_{i}\right),
$$

onde $\ell^{*}\left(\beta, \sigma_{e}^{2} ; W_{i}, Y_{i}\right)$, foi definido em (2.9), e $\beta=\left(\beta_{0}, \beta_{1}, \ldots, \beta_{p}\right)^{t}$. Observe-se que, neste caso o modelo não perturbado é obtido ao considerar $v_{i}=\mathbf{1}_{\mathbf{n}}$.

Derivando (2.61) com respeito a $v^{t}$ temos que

$$
\frac{\partial}{\partial v^{t}} \ell^{*}\left(\beta, v, \sigma_{e}^{2} ; \mathbf{W}, \mathbf{Y}\right)=\left(\ell^{*}\left(\beta, v, \sigma_{e}^{2} ; W_{1}, Y_{1}\right), \ldots, \ell^{*}\left(\beta, v, \sigma_{e}^{2} ; W_{n}, Y_{n}\right)\right)
$$

logo derivando com respeito a $\theta$, sendo $\theta=\left(\beta, \sigma_{e}^{2}\right)^{t}$, temos que

$$
\begin{aligned}
\Delta & =\frac{\partial}{\partial \theta \partial v^{t}} \ell^{*}\left(\beta, v, \sigma_{e}^{2} ; \mathrm{W}, \mathbf{Y}\right) \\
& =\frac{\partial}{\partial \theta}\left(\ell^{*}\left(\beta, v, \sigma_{e}^{2} ; W_{1}, Y_{1}\right), \ldots, \ell^{*}\left(\beta, v, \sigma_{e}^{2} ; W_{n}, Y_{n}\right)\right), \\
& =\left(\Delta_{1}, \ldots, \Delta_{n}\right)
\end{aligned}
$$

onde

$$
\Delta_{i}=\left(\begin{array}{c}
\frac{1}{\sigma_{e}^{2}}\left(Y_{i}-\sum_{j=0}^{p} \beta_{j} t_{i,(j)}\right) \\
\frac{1}{\sigma_{e}^{2}}\left(Y_{i} t_{i,(1)}-\sum_{j=0}^{p} \beta_{j} t_{i,(j+1)}\right) \\
\vdots \\
\frac{1}{\sigma_{e}^{2}}\left(Y_{i} t_{i,(p)}-\sum_{j=0}^{p} \beta_{j} t_{i,(j+p)}\right) \\
-\frac{1}{2 \sigma_{e}^{2}}+\frac{1}{2 \sigma_{e}^{4}}\left(Y_{i}^{2}+\sum_{j=0}^{p} \beta_{j}^{2} t_{i,(2 j)}-2 Y_{i} \sum_{j=0}^{p} \beta_{j} t_{i,(j)}+2 \sum_{j=0}^{p} \sum_{s=0}^{p} \beta_{j} \beta_{s} t_{i,(j+s)}\right)
\end{array}\right)
$$


para $j, s=0,1, \ldots, p, p$ representa ao total dos parâmetros $\beta$ em estudo. A direção $d_{\text {max }}$ é obtida a partir do maior valor autovalor absoluto da expressão (2.60).

\section{b) Perturbação da variável resposta}

Pode-se considerar dois tipos diferentes de perturbação da $i$-ésima observação, ou seja,

$$
\begin{gathered}
Y_{i}\left(v_{i}\right)=Y_{i}+v_{i}, \\
Y_{i}\left(v_{i}\right)=Y_{i} v_{i}, \quad i=1,2, \ldots, n .
\end{gathered}
$$

No primeiro caso se diz que a perturbação é aditiva e no segundo caso a perturbação é multiplicativa. O logaritmo da função verossimilhança corrigida perturbada da $i$-ésima observação é dado por,

$$
\begin{aligned}
\ell^{*}\left(\beta, \sigma_{e}^{2}, v_{i} ; W_{i}, Y_{i}\right)= & -\frac{1}{2} \log (2 \pi)-\frac{1}{2} \log \left(\sigma_{e}^{2}\right)-\frac{1}{2 \sigma_{e}^{2}}\left[Y_{i}^{2}\left(v_{i}\right)+\sum_{j=0}^{p} \beta_{j}^{2} t_{i,(2 j)}\right. \\
& \left.-2 Y_{i}\left(v_{i}\right) \sum_{j=0}^{p} \beta_{j} t_{i,(j)}+2 \sum_{j=0}^{p} \sum_{s=0}^{p} \beta_{j} \beta_{s} t_{i,(j+s)}\right] .
\end{aligned}
$$

Derivando (2.66) com respeito a $v_{i}$ obtemos

$$
\frac{\partial}{\partial v_{i}}\left(\beta, v_{i}, \sigma_{e}^{2} ; W_{i}, Y_{i}\right)=-\frac{1}{2 \sigma_{e}^{2}}\left[\frac{\partial Y_{i}^{2}\left(v_{i}\right)}{\partial v_{i}}-2 \sum_{j=0}^{p} \beta_{j} t_{i,(j)} \frac{\partial Y_{i}\left(v_{i}\right)}{\partial v_{i}}\right] .
$$

Caso da perturbação aditiva, para esta situação consideramos a expressão (2.64), então a expressão acima fica

$$
\frac{\partial}{\partial v_{i}} \ell^{*}\left(\beta, v_{i}, \sigma_{e}^{2} ; W_{i}, Y_{i}\right)=-\frac{1}{2 \sigma_{e}^{2}}\left[2\left(Y_{i}+v_{i}\right)-2 \sum_{j=0}^{p} \beta_{j} t_{i,(j)}\right],
$$

logo considerando a expressão (2.61), um vetor que representa a cada $\Delta_{i}$ é dado por: 


$$
\Delta_{i}=\left(\begin{array}{c}
\frac{1}{\sigma_{e}^{2}} \\
\frac{1}{\sigma_{e}^{2}} t_{i,(1)} \\
\vdots \\
\frac{1}{\sigma_{e}^{2}} t_{i,(p)}^{p} \\
\frac{1}{\sigma_{e}^{4}}\left(Y_{i}+v_{i}-\sum_{j=0}^{p} \beta_{j} t_{i,(j)}\right)
\end{array}\right)
$$

Caso da perturbação multiplicativa, neste caso é considerada a expressão (2.65), e a expressão (2.67) fica como

$$
\frac{\partial}{\partial v_{i}} \ell^{*}\left(\beta, v_{i}, \sigma_{e}^{2} ; W_{i}, Y_{i}\right)=-\frac{1}{2 \sigma_{e}^{2}}\left[2 Y_{i}^{2} v_{i}-2 Y_{i} \sum_{j=0}^{p} \beta_{j} t_{i,(j)}\right],
$$

considerando a expressão (2.61), um vetor que representa a cada $\Delta_{i}$ é dado por:

$$
\Delta_{i}=\left(\begin{array}{c}
\frac{1}{\sigma_{e}^{2}} Y_{i} \\
\frac{1}{\sigma_{e}^{2}} Y_{i} t_{i,(1)} \\
\vdots \\
\frac{1}{\sigma_{e}^{2}} Y_{i} t_{i,(p)} \\
\frac{1}{\sigma_{e}^{4}}\left(Y_{i}^{2} v_{i}-Y_{i} \sum_{j=0}^{p} \beta_{j} t_{i,(j)}\right)
\end{array}\right) .
$$

c) Perturbação das variâncias dos erros.

Nesta seção pretende-se estudar o caso de perturbações da homogeneidade de variâncias, considerando um modelo em que

$$
\operatorname{Var}\left(e_{i}\right)=\frac{\sigma_{e}^{2}}{v_{i}}
$$

onde $v_{i}>0, i=1,2, \ldots, n$. O logaritmo da função verossimilhança corrigida perturbada para a $i$-ésima observação é dado por 


$$
\begin{aligned}
\ell^{*}\left(\beta, \sigma_{e}^{2}, v_{i} ; W_{i}, Y_{i}\right)= & -\frac{1}{2} \log (2 \pi)-\frac{1}{2} \log \left(\sigma_{e}^{2}\right)+\frac{1}{2} \log \left(v_{i}\right)-\frac{v_{i}}{2 \sigma_{e}^{2}}\left[Y_{i}^{2}+\sum_{j=0}^{p} \beta_{j}^{2} t_{i,(2 j)}\right. \\
& \left.-2 Y_{i} \sum_{j=0}^{p} \beta_{j} t_{i,(j)}+2 \sum_{j=0}^{p} \sum_{\substack{j=0 \\
j<s}}^{p} \beta_{j} \beta_{s} t_{i,(j+s)}\right] .
\end{aligned}
$$

Derivando a expressão (2.71) em função a $v_{i}$ temos

$$
\begin{aligned}
\frac{\partial}{\partial v_{i}} \ell^{*}\left(\beta, \sigma_{e}^{2}, v_{i} ; W_{i}, Y_{i}\right)= & \frac{1}{2 v_{i}}-\frac{1}{2 \sigma_{e}^{2}}\left[Y_{i}^{2}+\sum_{j=0}^{p} \beta_{j}^{2} t_{i,(2 j)}\right. \\
& \left.-2 Y_{i} \sum_{j=0}^{p} \beta_{j} t_{i,(j)}+2 \sum_{\substack{j=0 \\
j<s}}^{p} \sum_{\substack{j=0 \\
j<s}}^{p} \beta_{j} \beta_{s} t_{i,(j+s)}\right],
\end{aligned}
$$

considerando a expressão (2.61), um vetor que representa a cada $\Delta_{i}$ é dado por

$$
\Delta_{i}=\left(\begin{array}{c}
\frac{1}{\sigma_{e}^{2}}\left(Y_{i}-\sum_{j=0}^{p} \beta_{j} t_{i,(j)}\right) \\
\frac{1}{\sigma_{e}^{2}}\left(Y_{i} t_{i,(1)}-\sum_{j=0}^{p} \beta_{j} t_{i,(j+1)}\right) \\
\vdots \\
\frac{1}{\sigma_{e}^{2}}\left(Y_{i} t_{i,(p)}-\sum_{j=0}^{p} \beta_{j} t_{i,(j+p)}\right) \\
\frac{1}{2 \sigma_{e}^{4}}\left(Y_{i}^{2}+\sum_{j=0}^{p} \beta_{j}^{2} t_{i,(2 j)}-2 Y_{i} \sum_{j=0}^{p} \beta_{j} t_{i,(j)}+2 \sum_{j=0}^{p} \sum_{s=0}^{p} \beta_{j} \beta_{s} t_{i,(j+s)}\right)
\end{array}\right)
$$




\section{d) Perturbação da covariável.}

Estudamos dois tipos diferentes de perturbação da $i$-ésima observação, ou seja,

$$
\begin{gathered}
W_{i}\left(v_{i}\right)=W_{i}+v_{i}, \\
W_{i}\left(v_{i}\right)=W_{i} v_{i}, \quad i=1,2, \ldots, n .
\end{gathered}
$$

No primeiro caso se diz que a perturbação das covariáveis é aditiva e no segundo caso a perturbação das covariáveis é multiplicativa. O logaritmo da função verossimilhança corrigida perturbada da i-ésima observação é dado por

$$
\begin{aligned}
\ell^{*}\left(\beta, v_{i}, \sigma_{e}^{2} ; W_{i}, Y_{i}\right)= & -\frac{1}{2} \log (2 \pi)-\frac{1}{2} \log \left(\sigma_{e}^{2}\right)-\frac{1}{2 \sigma_{e}^{2}}\left[Y_{i}^{2}+\sum_{j=0}^{p} \beta_{j}^{2} t_{i,(2 j)}\left(v_{i}\right)\right. \\
& \left.-2 Y_{i} \sum_{j=0}^{p} \beta_{j} t_{i,(j)}\left(v_{i}\right)+2 \sum_{\substack{j=0 \\
j<s}}^{p} \sum_{\substack{s=0 \\
j<s}}^{p} \beta_{j} \beta_{s} t_{i,(j+s)}\left(v_{i}\right)\right]
\end{aligned}
$$

sendo que

$$
t_{i,(j+1)}\left(v_{i}\right)=W_{i}\left(v_{i}\right) t_{i,(j)}\left(v_{i}\right)-j \sigma_{u}^{2} t_{i,(j-1)}\left(v_{i}\right)
$$

derivando a expressão (2.76) em função a $v_{i}$ temos

$$
\begin{aligned}
\frac{\partial}{\partial v_{i}} \ell^{*}\left(\beta, v_{i}, \sigma_{e}^{2} ; W_{i}, Y_{i}\right)= & -\frac{1}{2 \sigma_{e}^{2}}\left[\sum_{j=0}^{p} \beta_{j}^{2} \frac{\partial t_{i,(2 j)}\left(v_{i}\right)}{\partial v_{i}}-2 Y_{i} \sum_{j=0}^{p} \beta_{j} \frac{\partial t_{i,(2 j)}\left(v_{i}\right)}{\partial v_{i}}\right. \\
& \left.+2 \sum_{\substack{j=0 \\
s<j}}^{p} \sum_{\substack{s=0 \\
s}}^{p} \beta_{j} \beta_{j} \frac{\partial t_{i,(j+s)}\left(v_{i}\right)}{\partial v_{i}}\right] .
\end{aligned}
$$

Considerando a expressão (2.61), um vetor que representa a cada $\Delta_{i}$ é dado por, 


$$
\Delta_{i}=\left(\begin{array}{c}
\frac{1}{\sigma_{e}^{2}}\left[Y_{i}-\sum_{j=0}^{p} \beta_{j} \frac{\partial t_{i,(j)}\left(v_{i}\right)}{\partial v_{i}}\right] \\
\frac{1}{\sigma_{e}^{2}}\left[Y_{i} \frac{\partial t_{i,(1)}\left(v_{i}\right)}{\partial v_{i}}-\sum_{j=0}^{p} \beta_{j} \frac{\partial t_{i,(j+1)}\left(v_{i}\right)}{\partial v_{i}}\right] \\
\vdots \\
\frac{1}{\sigma_{e}^{2}}\left[Y_{i} \frac{\partial t_{i,(p)}\left(v_{i}\right)}{\partial v_{i}}-\sum_{j=0}^{p} \beta_{j} \frac{\partial t_{i,(j+p)}\left(v_{i}\right)}{\partial v_{i}}\right] \\
\frac{1}{2 \sigma_{e}^{4}}\left[Y_{i}^{2}+\sum_{j=0}^{p} \beta_{j}^{2} \frac{\partial t_{i,(2 j)}\left(v_{i}\right)}{\partial v_{i}}-2 Y_{i} \sum_{j=0}^{p} \beta_{j} \frac{\partial t_{i,(j)}\left(v_{i}\right)}{\partial v_{i}}+2 \sum_{j=0}^{p} \sum_{s=0}^{p} \beta_{j} \beta_{s} \frac{\partial t_{i,(j+s)}\left(v_{i}\right)}{\partial v_{i}}\right]
\end{array}\right) .
$$

As derivadas na expressão acima terá a forma seguinte, se estamos frente a um esquema aditivo

$$
\frac{\partial t_{i,(m)}\left(v_{i}\right)}{\partial v_{i}}=m t_{i,(m-1)}\left(v_{i}\right) \quad m=1,2 \ldots, s,
$$

enquanto se estamos frente a um esquema multiplicativo, as derivadas terão a forma

$$
\frac{\partial t_{i,(m)}\left(v_{i}\right)}{\partial v_{i}}=m W_{i} t_{i,(m-1)}\left(v_{i}\right) \quad m=1,2 \ldots, s .
$$

\subsubsection{Aplicação}

Selecionamos o exemplo apresentado por Fuller, (1987) onde se tem 120 observações (pag. 266). Adotamos um modelo quadrático, e assumindo $\sigma_{u}^{2}=0,09$ em nossa análise.

O logaritmo da função verossimilhança corrigida para um modelo quadrático, de acordo com (2.9) é expresso como

$$
\begin{aligned}
\ell^{*}\left(\beta, \sigma_{e}^{2} ; \mathrm{Y}, \mathrm{W}\right)= & -\frac{n}{2} \log (2 \pi)-\frac{n}{2} \log \left(\sigma_{e}^{2}\right)-\frac{1}{2 \sigma_{e}^{2}} \sum_{i=1}^{n}\left[Y_{i}^{2}+\beta_{0}^{2}+\beta_{1}^{2} t_{i,(2)}+\beta_{2}^{2} t_{i,(4)}-2 Y_{i} \beta_{0}\right. \\
& \left.-2 Y_{i} t_{i,(1)}-2 Y_{i} t_{i,(2)}+2 \beta_{0} \beta_{1} t_{i,(1)}+2 \beta_{0} \beta_{2} t_{i,(2)}+2 \beta_{1} \beta_{2} t_{i,(3)}\right],
\end{aligned}
$$

para $i=1, \ldots, n$. As matrizes $\Delta_{i}$ nos diferentes esquemas de perturbação para este modelo são apresentados na seqüência. 
Esquema 1: Perturbação de ponderação dos Casos

$$
\begin{aligned}
\Delta_{i, 0}= & \frac{1}{\sigma_{e}^{2}}\left[Y_{i}-\beta_{0}-\beta_{1} t_{i,(1)}-\beta_{2} t_{i,(2)}\right], \\
\Delta_{i, 1}= & \frac{1}{\sigma_{e}^{2}}\left[Y_{i} t_{i,(1)}-\beta_{0} t_{i,(1)}-\beta_{1} t_{i,(2)}-\beta_{2} t_{i,(3)}\right], \\
\Delta_{i, 2}= & \frac{1}{\sigma_{e}^{2}}\left[Y_{i} t_{i,(2)}-\beta_{0} t_{i,(2)}-\beta_{1} t_{i,(3)}-\beta_{2} t_{i,(4)}\right], \\
\Delta_{i, 3}= & -\frac{1}{2 \sigma_{e}^{2}}+\frac{1}{2 \sigma_{e}^{4}}\left[Y_{i}^{2}+\beta_{0}^{2}+\beta_{1}^{2} t_{i,(2)}+\beta_{2}^{2} t_{i,(4)}-2 Y_{i} \beta_{0}\right. \\
& \left.-2 Y_{i} t_{i,(1)}-2 Y_{i} t_{i,(2)}+2 \beta_{0} \beta_{1} t_{i,(1)}+2 \beta_{0} \beta_{2} t_{i,(2)}+2 \beta_{1} \beta_{2} t_{i,(3)}\right],
\end{aligned}
$$

para $i=1, \ldots, n$.

Esquema 2: Perturbação da variável resposta

Situação A: Perturbação aditiva .

$$
\begin{aligned}
& \Delta_{i, 0}=\frac{1}{\sigma_{e}^{2}} \\
& \Delta_{i, 1}=\frac{1}{\sigma_{e}^{2}} t_{i,(1)}, \\
& \Delta_{i, 2}=\frac{1}{\sigma_{e}^{2}} t_{i,(2)}, \\
& \Delta_{i, 3}=\frac{1}{\sigma_{e}^{4}}\left[v_{i}+Y_{i}-\beta_{0}-\beta_{1} t_{i,(1)}-\beta_{2} t_{i,(2)}\right],
\end{aligned}
$$

para $i=1, \ldots, n$.

Situação B: Perturbação multiplicativa

$$
\begin{aligned}
\Delta_{i, 0} & =\frac{1}{\sigma_{e}^{2}} Y_{i}, \\
\Delta_{i, 1} & =\frac{1}{\sigma_{e}^{2}} Y_{i} t_{i,(1)}, \\
\Delta_{i, 2} & =\frac{1}{\sigma_{e}^{2}} Y_{i} t_{i,(2)}, \\
\Delta_{i, 3} & =\frac{Y_{i}}{\sigma_{e}^{4}}\left[Y_{i} v_{i}-\beta_{0}-\beta_{1} t_{i,(1)}-\beta_{2} t_{i,(2)}\right],
\end{aligned}
$$

para $i=1, \ldots, n$. 
Esquema 3: Perturbação das variâncias dos erros

$$
\begin{aligned}
\Delta_{i, 0}= & \frac{1}{\sigma_{e}^{2}}\left[Y_{i}-\beta_{0}-\beta_{1} t_{i,(1)}-\beta_{2} t_{i,(2)}\right] \\
\Delta_{i, 1}= & \frac{1}{\sigma_{e}^{2}}\left[Y_{i} t_{i,(1)}-\beta_{0} t_{i,(1)}-\beta_{1} t_{i,(2)}-\beta_{2} t_{i,(3)}\right] \\
\Delta_{i, 2}= & \frac{1}{\sigma_{e}^{2}}\left[Y_{i} t_{i,(2)}-\beta_{0} t_{i,(2)}-\beta_{1} t_{i,(3)}-\beta_{2} t_{i,(4)}\right] \\
\Delta_{i, 3}= & \frac{1}{2 \sigma_{e}^{4}}\left[Y_{i}^{2}+\beta_{0}^{2}+\beta_{1}^{2} t_{i,(2)}+\beta_{2}^{2} t_{i,(4)}-2 Y_{i} \beta_{0}\right. \\
& \left.-2 Y_{i} t_{i,(1)}-2 Y_{i} t_{i,(2)}+2 \beta_{0} \beta_{1} t_{i,(1)}+2 \beta_{0} \beta_{2} t_{i,(2)}+2 \beta_{1} \beta_{2} t_{i,(3)}\right],
\end{aligned}
$$

para $i=1, \ldots, n$.

\section{Esquema 4: Perturbação da covariáveis}

\section{Situação A: Perturbação aditiva}

Para este tipo de esquema aditivo consideramos a expressão (2.79), obtendo

$$
\begin{aligned}
\Delta_{i, 0}= & -\frac{1}{\sigma_{e}^{2}}\left[\beta_{1}+2 \beta_{2} t_{i,(1)}\left(v_{i}\right)\right] \\
\Delta_{i, 1}= & \frac{1}{\sigma_{e}^{2}}\left[Y_{i}-\beta_{0}-2 \beta_{1} t_{i,(1)}\left(v_{i}\right)-3 \beta_{2} t_{i,(2)}\left(v_{i}\right)\right] \\
\Delta_{i, 2}= & \frac{1}{\sigma_{e}^{2}}\left[2 Y_{i} t_{i,(1)}-2 \beta_{0} t_{i,(1)}-3 \beta_{1} t_{i,(2)}-4 \beta_{2} t_{i,(3)}\right], \\
\Delta_{i, 3}= & \frac{1}{\sigma_{e}^{4}}\left[Y_{i} \beta_{1}+2 Y_{i} \beta_{2} t_{i,(1)}\left(v_{i}\right)-\beta_{1}^{2} t_{i,(1)}\left(v_{i}\right)-2 \beta_{2}^{2} t_{i,(3)}\left(v_{i}\right)-\beta_{0} \beta_{1}\right. \\
& \left.-2 \beta_{0} \beta_{2} t_{i,(1)}\left(v_{i}\right)-3 \beta_{1} \beta_{2} t_{i,(2)}\left(v_{i}\right)\right] .
\end{aligned}
$$

para $i=1, \ldots, n$.

\section{Situação B: Perturbação multiplicativa.}

Para este tipo de esquema multiplicativo consideramos a expressão (2.80), obtendo

$$
\begin{aligned}
& \Delta_{i, 0}=-\frac{1}{\sigma_{e}^{2}}\left[\beta_{1}+2 \beta_{2} W_{i} t_{i,(1)}\left(v_{i}\right)\right], \\
& \Delta_{i, 1}=\frac{1}{\sigma_{e}^{2}}\left[Y_{i} W_{i}-\beta_{0}-2 \beta_{1} W_{i} t_{i,(1)}\left(v_{i}\right)-3 \beta_{2} W_{i} t_{i,(2)}\left(v_{i}\right)\right],
\end{aligned}
$$




$$
\begin{aligned}
\Delta_{i, 2}= & \frac{1}{\sigma_{e}^{2}}\left[2 Y_{i} W_{i} t_{i,(1)}-2 \beta_{0} W_{i} t_{i,(1)}-3 \beta_{1} W_{i} t_{i,(2)}-4 \beta_{2} W_{i} t_{i,(3)}\right] \\
\Delta_{i, 3}= & \frac{1}{\sigma_{e}^{4}}\left[Y_{i} W_{i} \beta_{1}+2 Y_{i} \beta_{2} t_{i,(1)}\left(v_{i}\right)-\beta_{1}^{2} W_{i} t_{i,(1)}\left(v_{i}\right)-2 \beta_{2}^{2} W_{i} t_{i,(3)}\left(v_{i}\right)-\beta_{0} \beta_{1}\right. \\
& \left.-2 \beta_{0} \beta_{2} W_{i} t_{i,(1)}\left(v_{i}\right)-3 \beta_{1} \beta_{2} W_{i} t_{i,(2)}\left(v_{i}\right)\right] .
\end{aligned}
$$

para $i=1, \ldots, n$.

Para esta aplicação prática considerarmos os diferentes esquemas de perturbação com a finalidade de obter os pontos que se forem perturbados podem afetar os resultados obtidos. Apresentamos os gráficos de índice da direção $d_{\max }$ nos diversos esquemas.

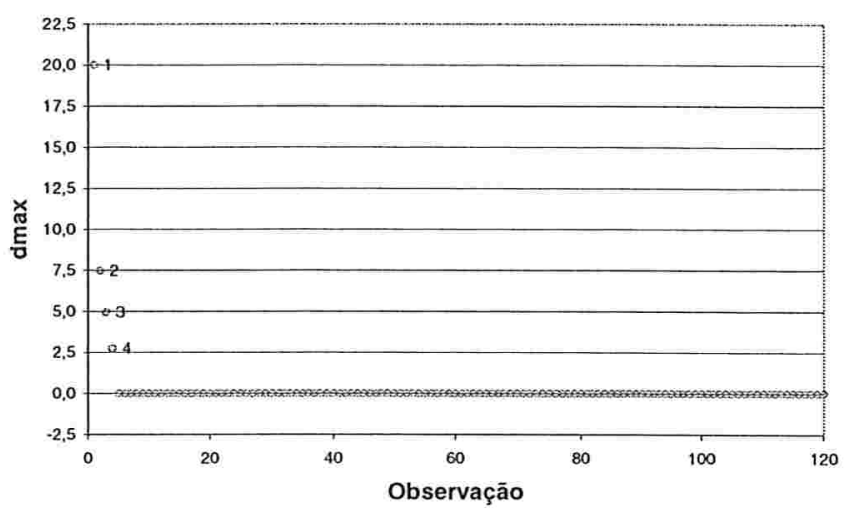

Fig. 2.8. Gráfico de índice segundo o esquema de perturbação da ponderação dos casos 


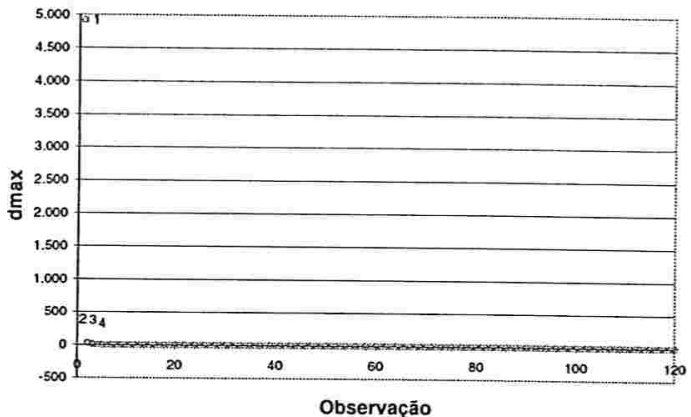

(a) Caso aditivo

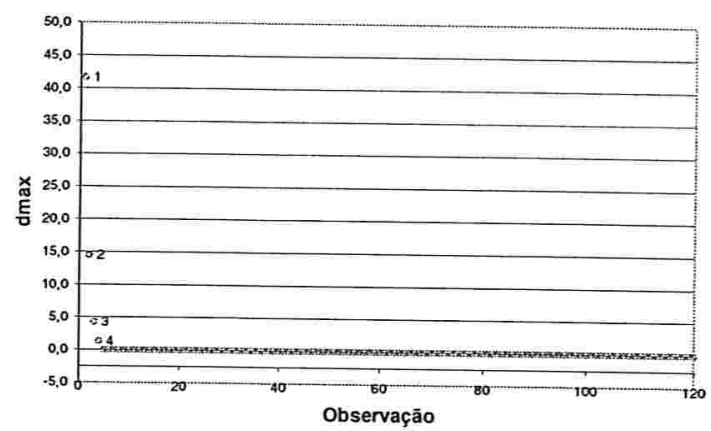

(b) Caso multiplicativo

Fig. 2.9. Gráfico de índice segundo o esquema de perturbação da variável resposta

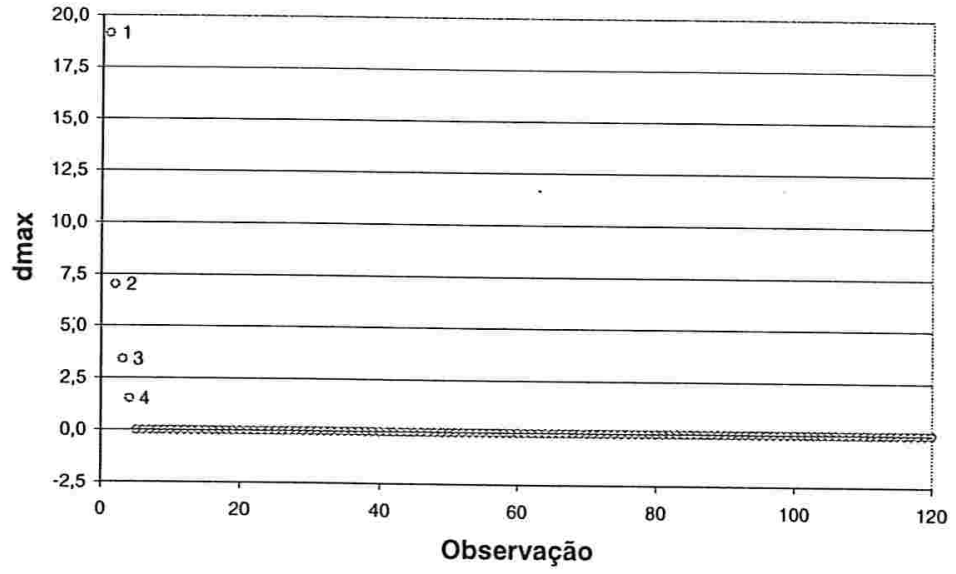

Fig. 2.10. Gráfico de índice segundo o esquema perturbação das variâncias dos erros de medição da variável resposta 


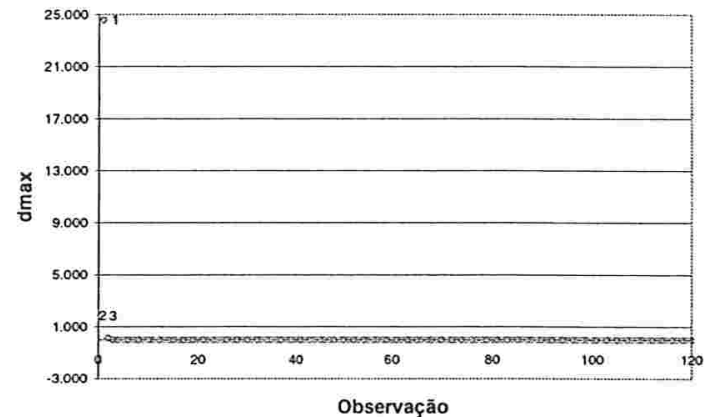

(a) Caso aditivo

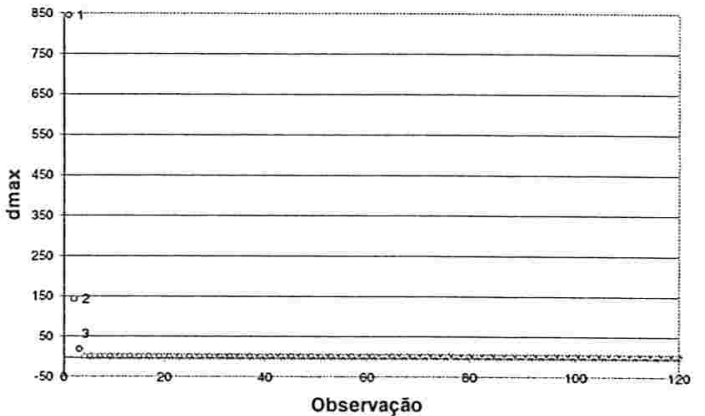

(b) Caso multiplicativo

Fig. 2.11. Gráfico de índice segundo o esquema de perturbação da covariável

Dos gráficos acima notamos que os pontos com valores de $d_{\max }$ mais altos se apresentam nas primeiras quatro observações. Para observar se estes pontos poderia afetar o comportamento do modelo faremos uma análise confirmatória com a finalidade de avaliar quais pontos são influentes. Esta análise confirmatória é apresentada na seguinte tabela: 
Tabela 2.7. Índice de variação percentual nos parâmetros em um modelo quadrático devido aos pontos 1, 2, 3 e 4

\begin{tabular}{|c|c|c|c|c|c|}
\hline Pontos & $\beta_{0}$ & $\beta_{1}$ & $\beta_{2}$ & $\sigma_{e}^{2}$ & Comparação \\
\hline 1 & 6,24 & 1,99 & 1,26 & 2,85 & 0,83 \\
2 & 22,46 & 0,17 & 0,75 & 1,94 & 0,83 \\
3 & 9,59 & 2,49 & 0,96 & 5,97 & 0,83 \\
4 & 24,29 & 0,64 & 0,62 & 2,72 & 0,83 \\
1,2 & 17,45 & 2,16 & 0,47 & 4,84 & 1,67 \\
1,3 & 10,89 & 0,67 & 0,02 & 2,84 & 1,67 \\
1,4 & 17,53 & 2,71 & 0,71 & 5,56 & 1,67 \\
2,3 & 11,98 & 2,37 & 1,69 & 4,06 & 1,67 \\
2,4 & 46,46 & 0,81 & 1,35 & 4,66 & 1,67 \\
3,4 & 15,10 & 1,94 & 1,63 & 3,21 & 1,67 \\
$1,2,3$ & 11,83 & 0,54 & 0,75 & 0,87 & 2,50 \\
$1,2,4$ & 40,93 & 2,90 & 0,06 & 7,55 & 2,50 \\
$1,3,4$ & 13,47 & 0,05 & 0,60 & 0,07 & 2,50 \\
$2,3,4$ & 36,36 & 1,81 & 2,35 & 1,30 & 2,50 \\
$1,2,3,4$ & 35,88 & 0,08 & 1,37 & 1,90 & 3,33 \\
\hline
\end{tabular}

Observemos na tabela acima, que os pontos 1 e 3 são pontos que influem o comportamento dos parâmetros do modelo. 


\subsection{Caso 3: Variâncias heterogêneas}

\subsubsection{Metodologia}

Nesta seção apresentaremos um método de estimação similar ao apresentado no Caso 1, com a diferença que as variâncias consideradas para cada observação são conhecidas e além disso diferentes de observação para observação. Este tipo de situação não foi muito estudada na literatura estatística. Um estudo de um caso similar em modelos de grau 1 foi Galea-Rojas, de Castilho, Bolfarine e de Castro (2003), que analisaram o modelo de regressão proposto por Ripley e Thompson (1987) e propuseram um algoritmo tipo EM para estimar os parâmetros. Em nosso caso estudaremos os estimadores segundo a proposta de Nakamura (1990), isto é, a partir do logaritmo da função verossimilhança corrigida.

Para esta seção pode-se considerar um modelo funcional com suposições do tipo

$$
\left(\begin{array}{c}
e_{i} \\
u_{i}
\end{array}\right) \sim N_{2}\left[\left(\begin{array}{l}
0 \\
0
\end{array}\right) ;\left(\begin{array}{cc}
\sigma_{e_{i}}^{2} & 0 \\
0 & \sigma_{u_{i}}^{2}
\end{array}\right)\right] \text {, independentes, } i=1, \ldots, n,
$$

ou pode-se considerar o modelo estrutural com suposições do tipo,

$$
\left(\begin{array}{c}
X_{i} \\
e_{i} \\
u_{i}
\end{array}\right) \sim N_{3}\left[\left(\begin{array}{c}
\mu_{x} \\
0 \\
0
\end{array}\right) ;\left(\begin{array}{ccc}
\sigma_{x}^{2} & 0 & 0 \\
0 & \sigma_{e_{i}}^{2} & 0 \\
0 & 0 & \sigma_{u_{i}}^{2}
\end{array}\right)\right] \text { independentes, }
$$

$i=1, \ldots, n$. Como foi dito acima, $\sigma_{e_{i}}^{2}$ e $\sigma_{u_{i}}^{2}$ são conhecidas, de modo que o logaritmo da função verossimilhança corrigida pode ser obtido como na seção 2.2 , levando a expressão que pode ser funcional ou estrutural

$$
\begin{aligned}
\ell^{*}(\beta ; \mathrm{Y}, \mathrm{W})= & -\frac{n}{2} \log (\pi)-\frac{1}{2} \log \left(\sum_{i=1}^{n} \sigma_{e_{i}}^{2}\right)-\frac{1}{2} \sum_{i=1}^{n} \frac{1}{\sigma_{e_{i}}^{2}}\left(Y_{i}^{2}+\sum_{j=0}^{p} \beta_{j}^{2} t_{i,(2 j)}\right. \\
& \left.-2 Y_{i} \sum_{j=0}^{p} \beta_{j} t_{i,(j)}+2 \sum_{\substack{j=0 \\
s<j}}^{p} \sum_{\substack{s=0 \\
s<j}}^{p} \beta_{j} \beta_{s} t_{i,(j+s)}\right) .
\end{aligned}
$$


A partir desta expressão podemos obter a função escore corrigido, derivando-a em relação a cada parâmetro $\beta$, resultando em

$$
U^{*}\left(\beta_{s}\right)=\sum_{i=1}^{n} \frac{1}{\sigma_{e_{i}}^{2}}\left(Y_{i} t_{i,(s)}-\sum_{j=0}^{p} \beta_{j} t_{i,(j+s)}\right),
$$

onde $s=0,1,2, \ldots, p$. Enquanto que os elementos de $t_{i,(s)}$ sofrem uma pequena mudança em relação à expressão apresentada em (2.27), passando a ser

$$
t_{i,(j+1)}=W_{i} t_{i,(j)}-j \sigma_{u_{i}}^{2} t_{i,(j-1)}
$$

$i=1, \ldots, n, j=1, \ldots, p$.

A partir da função escore corrigido podemos obter as equações lineares que geram após alguns cálculos algébricos os estimadores para os parâmetros $\beta$, dados por

$$
\left(\begin{array}{cccc}
\sum_{i=1}^{n} \frac{1}{\sigma_{e_{i}}^{2}} & \sum_{i=1}^{n} \frac{t_{i,(1)}}{\sigma_{e_{i}}^{2}} & \ldots & \sum_{i=1}^{n} \frac{t_{i,(p)}}{\sigma_{e_{i}}^{2}} \\
\sum_{i=1}^{n} \frac{t_{i,(1)}}{\sigma_{e_{i}}^{2}} & \sum_{i=1}^{n} \frac{t_{i,(2)}}{\sigma_{e_{i}}^{2}} & \ldots & \sum_{i=1}^{n} \frac{t_{i,(p+1)}}{\sigma_{e_{i}}^{2}} \\
\vdots & \vdots & \ldots & \vdots \\
\sum_{i=1}^{n} \frac{t_{i,(p)}}{\sigma_{e_{i}}^{2}} & \sum_{i=1}^{n} \frac{t_{i,(p+1)}}{\sigma_{e_{i}}^{2}} & \ldots & \sum_{i=1}^{n} \frac{t_{i,(2 p)}}{\sigma_{e_{i}}^{2}}
\end{array}\right)\left(\begin{array}{c}
\beta_{0} \\
\beta_{1} \\
\vdots \\
\beta_{p}
\end{array}\right)=\left(\begin{array}{c}
\sum_{i=1}^{n} \frac{Y_{i}}{\sigma_{e_{i}}^{2}} \\
\sum_{i=1}^{n} \frac{Y_{i} t_{i,(1)}}{\sigma_{e_{i}}^{2}} \\
\vdots \\
\sum_{i=1}^{n} \frac{Y_{i} t_{i,(p)}}{\sigma_{e_{i}}^{2}}
\end{array}\right) .
$$

\subsubsection{Exemplos}

\section{Polinômio de primeiro grau}

Considerando a expressão $(2.86)$ e $m_{i}=\frac{1}{\sigma_{e_{i}}^{2}}$, para $\mathrm{p}=1$. temos

$$
\left(\begin{array}{cc}
\sum_{i=1}^{n} m_{i} & \sum_{i=1}^{n} m_{i} t_{i,(1)} \\
\sum_{i=1}^{n} m_{i} t_{i,(1)} & \sum_{i=1}^{n} m_{i} t_{i,(2)}
\end{array}\right)\left(\begin{array}{l}
\hat{\beta}_{0} \\
\hat{\beta}_{1}
\end{array}\right)=\left(\begin{array}{c}
\sum_{i=1}^{n} m_{i} Y_{i} \\
\sum_{i=1}^{n} m_{i} Y_{i} t_{i,(1)}
\end{array}\right) .
$$

Após alguns cálculos algébricos, obtemos

$$
\hat{\beta}_{0}=\frac{\sum_{i=1}^{n} m_{i} Y_{i}}{\sum_{i=1}^{n} m_{i}}-\frac{\sum_{i=1}^{n} m_{i} t_{i,(1)}}{\sum_{i=1}^{n} m_{i}} \hat{\beta}_{1}
$$




$$
\hat{\beta}_{1}=\frac{\sum_{i=1}^{n} m_{i} \sum_{i=1}^{n} m_{i} Y_{i} t_{i,(1)}-\sum_{i=1}^{n} m_{i} t_{i,(1)} \sum_{i=1}^{n} m_{i} Y_{i}}{\sum_{i=1}^{n} m_{i} \sum_{i=1}^{n} m_{i} t_{i,(2)}-\left(\sum_{i=1}^{n} m_{i} t_{i,(1)}\right)^{2}} .
$$

levando em conta que

$$
\begin{aligned}
\sum_{i=1}^{n} m_{i} t_{i,(1)} & =\sum_{i=1}^{n} m_{i} W_{i} \\
\sum_{i=1}^{n} m_{i} t_{i,(2)} & =\sum_{i=1}^{n} m_{i} W_{i}^{2}-\sum_{i=1}^{n} m_{i} \sigma_{u_{i}}^{2}=\sum_{i=1}^{n} m_{i} S_{W W}+\frac{\left(\sum_{i=1}^{n} W_{i}\right)^{2}}{\sum_{i=1}^{n} m_{i}}-\sum_{i=1}^{n} m_{i} \sigma_{u_{i}}^{2}, \\
\sum_{i=1}^{n} m_{i} Y_{i} t_{i,(1)} & =\sum_{i=1}^{n} m_{i} Y_{i} W_{i}=\sum_{i=1}^{n} m_{i} S_{Y W}+\frac{\left(\sum_{i=1}^{n} m_{i} Y_{i}\right)\left(\sum_{i=1}^{n} m_{i} W_{i}\right)}{\sum_{i=1}^{n} m_{i}}
\end{aligned}
$$

onde

$$
\begin{aligned}
\left(\sum_{i=1}^{n} m_{i}\right) S_{W W} & =\sum_{i=1}^{n} m_{i} W_{i}^{2}-\frac{\left(\sum_{i=1}^{n} m_{i} W_{i}\right)^{2}}{\sum_{i=1}^{n} m_{i}} \\
\left(\sum_{i=1}^{n} m_{i}\right) S_{Y W} & =\sum_{i=1}^{n} m_{i} Y_{i} W_{i}-\frac{\left(\sum_{i=1}^{n} m_{i} Y_{i}\right)\left(\sum_{i=1}^{n} m_{i} W_{i}\right)}{\sum_{i=1}^{n} m_{i}}
\end{aligned}
$$

temos que

$$
\begin{gathered}
\hat{\beta}_{0}=\frac{\sum_{i=1}^{n} m_{i} Y_{i}}{\sum_{i=1}^{n} m_{i}}-\frac{\sum_{i=1}^{n} m_{i} W_{i}}{\sum_{i=1}^{n} m_{i}} \hat{\beta}_{1}, \\
\hat{\beta}_{1}=\frac{S_{Y W}}{S_{W W}-S u u},
\end{gathered}
$$


sendo que

$$
S u u=\frac{\sum_{i=1}^{n} m_{i} \sigma_{u_{i}}^{2}}{\sum_{i=1}^{n} m_{i}} .
$$

Conforme obtido por Castro, Castilho e Bolfarine.

\section{Polinômio de segundo grau}

Considerando a expressão $(2.86)$ e $m_{i}=\frac{1}{\sigma_{e_{i}}^{2}}$ para $\mathrm{p}=2$, temos

$$
\left(\begin{array}{ccc}
\sum_{i=1}^{n} m_{i} & \sum_{i=1}^{n} m_{i} t_{i,(1)} & \sum_{i=1}^{n} m_{i} t_{i,(2)} \\
\sum_{i=1}^{n} m_{i} t_{i,(1)} & \sum_{i=1}^{n} m_{i} t_{i,(2)} & \sum_{i=1}^{n} m_{i} t_{i,(3)} \\
\sum_{i=1}^{n} m_{i} t_{i,(2)} & \sum_{i=1}^{n} m_{i} t_{i,(3)} & \sum_{i=1}^{n} m_{i} t_{i,(4)}
\end{array}\right)\left(\begin{array}{l}
\hat{\beta}_{0} \\
\hat{\beta}_{1} \\
\hat{\beta}_{2}
\end{array}\right)=\left(\begin{array}{c}
\sum_{i=1}^{n} m_{i} Y_{i} \\
\sum_{i=1}^{n} m_{i} Y_{i} t_{i,(1)} \\
\sum_{i=1}^{n} m_{i} Y_{i} t_{i,(2)}
\end{array}\right) .
$$

Se definirmos os desvios

$$
\begin{aligned}
& \mathcal{A}=\sum_{i=1}^{n} m_{i} \sum_{i=1}^{n} m_{i} Y_{i} t_{i,(1)}-\left(\sum_{i=1}^{n} m_{i} Y_{i} \sum_{i=1}^{n} m_{i} t_{i,(1)}\right), \\
& \mathcal{B}=\sum_{i=1}^{n} m_{i} \sum_{i=1}^{n} m_{i} Y_{i} t_{i,(2)}-\left(\sum_{i=1}^{n} m_{i} Y_{i} \sum_{i=1}^{n} m_{i} t_{i,(2)}\right), \\
& \mathcal{C}=\sum_{i=1}^{n} m_{i} \sum_{i=1}^{n} m_{i} t_{i,(2)}-\left(\sum_{i=1}^{n} m_{i} t_{i,(1)}\right)^{2}, \\
& \mathcal{D}=\sum_{i=1}^{n} m_{i} \sum_{i=1}^{n} m_{i} t_{i,(3)}-\left(\sum_{i=1}^{n} m_{i} t_{i,(1)}\right)\left(\sum_{i=1}^{n} m_{i} t_{i,(2)}\right), \\
& \mathcal{E}=\sum_{i=1}^{n} m_{i} \sum_{i=1}^{n} m_{i} t_{i,(4)}-\left(\sum_{i=1}^{n} m_{i} t_{i,(2)}\right)^{2},
\end{aligned}
$$

temos, após alguns cálculos algébricos com o sistema de equações apresentado acima:

$$
\hat{\beta}_{0}=\frac{\sum_{i=1}^{n} m_{i} Y_{i}}{\sum_{i=1}^{n} m_{i}}-\frac{\sum_{i=1}^{n} m_{i} t_{i,(1)}}{\sum_{i=1}^{n} m_{i}} \hat{\beta}_{1}-\frac{\sum_{i=1}^{n} m_{i} t_{i,(2)}}{\sum_{i=1}^{n} m_{i}} \hat{\beta}_{2}
$$




$$
\begin{aligned}
& \hat{\beta}_{1}=\frac{1}{\mathcal{C}}\left(\mathcal{A}-\mathcal{D} \hat{\beta}_{2}\right), \\
& \hat{\beta}_{2}=\frac{\mathcal{C B}-\mathcal{D} \mathcal{A}}{\mathcal{E} \mathcal{C}-\mathcal{D}^{2}}
\end{aligned}
$$

levando em conta $(2.85)$ temos que $t_{i,(1)}=W_{i}, t_{i,(2)}=W_{i}^{2}-\sigma_{u_{i}}^{2}, \quad t_{i,(3)}=W_{i}^{3}-3 W_{i} \sigma_{u_{i}}^{2}$ e $t_{i,(4)}=W_{i}^{4}-6 W_{i}^{2} \sigma_{u_{i}}^{2}+3 \sigma_{u_{i}}^{4}$, os desvios podem ser expressos da seguinte maneira:

$$
\begin{aligned}
\mathcal{A}= & \left(\sum_{i=1}^{n} m_{i}\right)^{2} S_{Y W}, \quad \mathcal{B}=\left(\sum_{i=1}^{n} m_{i}\right)^{2}\left(S_{Y W^{2}}-S_{Y \sigma_{u}^{2}}\right) \\
\mathcal{C}= & \left(\sum_{i=1}^{n} m_{i}\right)^{2} S_{W W}-\sum_{i=1}^{n} m_{i} \sum_{i=1}^{n} m_{i} \sigma_{u_{i}}^{2}, \\
\mathcal{D}= & \left(\sum_{i=1}^{n} m_{i}\right)^{2}\left(S_{W W^{2}}-S_{W \sigma_{u}^{2}}\right)-2 \sum_{i=1}^{n} m_{i} \sum_{i=1}^{n} m_{i} W_{i} \sigma_{u_{i}}^{2} \\
\mathcal{E}= & \left(\sum_{i=1}^{n} m_{i}\right)^{2}\left(S_{W^{2} W^{2}}+S_{\sigma_{u}^{2} \sigma_{u}^{2}}\right)+2 \sum_{i=1}^{n} m_{i} \sum_{i=1}^{n} m_{i} \sigma_{u_{i}}^{4}- \\
& -6 \sum_{i=1}^{n} m_{i} \sum_{i=1}^{n} m_{i} W_{i}^{2} \sigma_{u_{i}}^{2}+2 \sum_{i=1}^{n} m_{i} W_{i}^{2} \sum_{i=1}^{n} m_{i} \sigma_{u_{i}}^{2} .
\end{aligned}
$$

Com estas novas expressões de $\mathcal{A}, \mathcal{B}, \mathcal{C}, \mathcal{D}$ e $\mathcal{E}$, podemos reescrever as expressões de $\hat{\beta}_{0}, \hat{\beta}_{1}$ e $\hat{\beta}_{2}$, tendo sido usada a notação abaixo

$$
\begin{aligned}
& \left(\sum_{i=1}^{n} m_{i}\right)^{2} S_{W^{2} W^{2}}^{*}=\sum_{i=1}^{n} m_{i} \sum_{i=1}^{n} m_{i} W_{i}^{4}-\left(\sum_{i=1}^{n} m_{i} W_{i}^{2}\right)^{2} \\
& \left(\sum_{i=1}^{n} m_{i}\right)^{2} S_{W W^{2}}^{*}=\sum_{i=1}^{n} m_{i} \sum_{i=1}^{n} m_{i} W_{i}^{3}-\left(\sum_{i=1}^{n} m_{i} W_{i}\right)\left(\sum_{i=1}^{n} m_{i} W_{i}^{2}\right) \\
& \left(\sum_{i=1}^{n} m_{i}\right)^{2} S_{W W}^{*}=\sum_{i=1}^{n} m_{i} \sum_{i=1}^{n} m_{i} W_{i}^{2}-\left(\sum_{i=1}^{n} m_{i} W_{i}\right)^{2} \\
& \left(\sum_{i=1}^{n} m_{i}\right)^{2} S_{Y W^{2}}^{*}=\sum_{i=1}^{n} m_{i} \sum_{i=1}^{n} m_{i} Y_{i} W_{i}^{2}-\left(\sum_{i=1}^{n} m_{i} Y_{i}\right)\left(\sum_{i=1}^{n} m_{i} W_{i}^{2}\right), \\
& \left(\sum_{i=1}^{n} m_{i}\right)^{2} S_{Y W}^{*}=\sum_{i=1}^{n} m_{i} \sum_{i=1}^{n} m_{i} Y_{i} W_{i}-\left(\sum_{i=1}^{n} m_{i} Y_{i}\right)\left(\sum_{i=1}^{n} m_{i} W_{i}\right)
\end{aligned}
$$




$$
\begin{aligned}
& \left(\sum_{i=1}^{n} m_{i}\right)^{2} S_{Y \sigma_{u}^{2}}^{*}=\sum_{i=1}^{n} m_{i} \sum_{i=1}^{n} m_{i} Y_{i} \sigma_{u_{i}}^{2}-\left(\sum_{i=1}^{n} m_{i} Y_{i}\right)\left(\sum_{i=1}^{n} m_{i} \sigma_{u_{i}}^{2}\right) \\
& \left(\sum_{i=1}^{n} m_{i}\right)^{2} S_{W \sigma_{u}^{2}}^{*}=\sum_{i=1}^{n} m_{i} \sum_{i=1}^{n} m_{i} W_{i} \sigma_{u_{i}}^{2}-\left(\sum_{i=1}^{n} m_{i} W_{i}\right)\left(\sum_{i=1}^{n} m_{i} \sigma_{u_{i}}^{2}\right) \\
& \left(\sum_{i=1}^{n} m_{i}\right)^{2} S_{\sigma_{u}^{2} \sigma_{u}^{2}}^{*}=\sum_{i=1}^{n} m_{i} \sum_{i=1}^{n} m_{i} \sigma_{u_{i}}^{4}-\left(\sum_{i=1}^{n} m_{i} \sigma_{u_{i}}^{2}\right)^{2} .
\end{aligned}
$$

\subsubsection{Obtenção da matriz de covariâncias Assintóticas}

Como a condição de que $E(\bullet \mid \mathrm{Y}, \mathrm{X})$ e $\frac{\partial}{\partial \theta}(\bullet)$ serem permutáveis é satisfeita, para obter a matriz de covariâncias assintótica, precisamos conhecer matriz de informação de Fisher, definida a continuação,

$$
\mathbf{I}^{*}(\beta ; \mathbf{W}, \mathbf{Y})=-\frac{\partial}{\partial \beta} E\left[\mathbf{U}_{n}^{*}(\beta ; \mathbf{W}, \mathbf{Y})\right]
$$

assim como um componente que é o produto das funções escores individuais, isto é,

$$
\mathbf{S}^{*}(\beta ; \mathbf{W}, \mathbf{Y})=\sum_{i=1}^{n} E\left[\mathbf{U}_{i}^{*}(\beta ; \mathbf{W}, \mathbf{Y}) \mathbf{U}_{i}^{*}(\beta ; \mathbf{W}, \mathbf{Y})^{t}\right]
$$

com a finalidade de criar uma matriz de covariâncias de tipo sandwich, que segue a continuação

$$
£^{*}(\beta ; \mathbf{W}, \mathbf{Y})=\frac{1}{n}\left(\mathbf{I}^{*}(\beta ; \mathbf{W}, \mathbf{Y})\right)^{-1} \mathbf{S}^{*}(\beta ; \mathbf{W}, \mathbf{Y})\left(\mathbf{I}^{*}(\beta ; \mathbf{W}, \mathbf{Y})\right)^{-1}
$$

e desta forma temos

$$
\sqrt{n}(\hat{\beta}-\beta) \stackrel{D}{\longrightarrow} N_{p+1}\left(0, £^{*}\right)
$$

\subsubsection{Teste de hipóteses para $\beta$}

É de interesse testar,

$$
\begin{aligned}
& H_{0}: \beta=\beta^{(0)}, \\
& H_{1}: \beta \neq \beta^{(0)},
\end{aligned}
$$

onde $\beta^{(0)}$ é um vetor p-dimensional sendo que $\sigma_{u_{i}}^{2}$ e $\sigma_{e_{i}}^{2}$ são conhecidas, $i=1, \ldots, n$. 
a) Teste da razão de verossimilhanças (Teste de wilks)

A estatística de teste da razão de verossimilhanças é definida por

$$
Q_{R V}=2\left\{\ell^{*}(\hat{\beta} ; \mathrm{Y}, \mathrm{W})-\ell^{*}\left(\beta^{(0)} ; \mathrm{Y}, \mathrm{W}\right)\right\},
$$

cuja distribuição assintótica sob $H_{0}$ é $\chi_{(d)}^{2}$, onde $d=\operatorname{dim}\left(H_{0} \cup H_{1}\right)-\operatorname{dim}\left(H_{0}\right)=p+1$, sendo $p+1$ o número de parâmetros que se deseja testar.

Substituindo a solução (2.86) em (2.93), levando em conta (2.83) temos

$$
\begin{aligned}
Q_{R V}= & -\sum_{i=1}^{n} \frac{1}{\sigma_{e_{i}}^{2}}\left[\sum_{j=0}^{p}\left(\hat{\beta}_{j}^{2}-\beta_{j}^{2(0)}\right) t_{i,(2 j)}-2 Y_{i} \sum_{j=0}^{p}\left(\hat{\beta}_{j}-\beta_{j}^{(0)}\right) t_{i,(j)}\right. \\
& \left.+2 \sum_{\substack{j=0 \\
j<s}}^{p} \sum_{\substack{s=0 \\
j<s}}\left(\hat{\beta}_{j} \hat{\beta}_{s}-\beta_{j}^{(0)} \beta_{s}^{(0)}\right) t_{i,(j+s)}\right]
\end{aligned}
$$

b) Teste de wald.

A estatística de teste de wald é definido por

$$
Q_{W}=\left(\hat{\beta}-\beta^{(0)}\right)^{t} £^{*}\left(\hat{\beta}-\beta^{(0)}\right)
$$

cuja distribuição assintótica sob $H_{0}$ é $\chi_{d}^{2}$, onde $£^{*}$ é segundo (2.92).

c) Teste de escore (Teste de rao).

A estatística de teste de escore é definido por

$$
Q_{E}=U^{*}\left(\beta^{(0)}\right)^{t} £^{*-1} U^{*}\left(\beta^{(0)}\right)
$$

cuja distribuição assintótica sob $H_{0}$ é $\chi_{d}^{2}$, onde $U^{*}(\beta)$ vem de (2.84) e $£^{*}$ é segundo (2.92).

\subsubsection{Aplicações numéricas}

Com a finalidade de aplicar a metodologia apresentada, consideramos dois tipos de aplicações. O primeiro será uma simulação de dados e a outra será uma aplicação prática.

a) Um estudo de simulação

Considerando um valor de $n$ fixo, os elementos do modelo quadrático podem ser definidos a partir de: 
- Gerar valores da forma $X_{i} \sim \operatorname{Binomial}(n ; 0,5)$,

- Gerar $n$ valores $u_{i}$ independentes com distribuição $N\left(0 ; \sigma_{u_{i}}^{2}\right)$,

- Gerar $n$ valores $e_{i}$ independentes com distribuição $N\left(0 ; \sigma_{e_{i}}^{2}\right)$,

- Os valores de $\sigma_{u_{i}}^{2}$ serão obtidos a partir de uma distribuição uniforme em $(0 ; 0,20)$, enquanto $\sigma_{e_{i}}^{2}$ serão obtidos a partir de uma distribuição uniforme em $(0 ; 0,5)$,

- Calcular $W_{i}=X_{i}+u_{i}$.

- Calcular $Y_{i}=\beta_{0}+\beta_{1} X_{i}+\beta_{1} X_{i}^{2}+e_{i}$, sendo os valores de $\beta_{0}, \beta_{1}$ e $\beta_{2}$ iguais a um, $i=1, \ldots, n$.

\section{Resultados}

Após implementar a metodologia apresentada na linguagem Ox (Doornik, 1998), temos os seguintes resultados experimentais que são apresentados em forma resumida nas tabelas abaixo. Apresentamos também os resultados de um modelo polinomial quadrático quando são usados estimadores ingênuos ("naive"). 
Tabela 2.8. Estimativas segundo o método "naive" para diferentes tamanhos de amostra em um modelo de regressão quadrática quando são consideradas variâncias heterogêneas.

\begin{tabular}{|c|c|c|c|c||c|c|c|}
\hline \multirow{2}{*}{$\mathrm{n}$} & \multirow{2}{*}{ Parâmetro } & \multicolumn{3}{|c||}{ Naive } & \multicolumn{3}{c|}{ Escore corrigido } \\
\cline { 2 - 8 } & & Estimativa & Desv.Pad. & EQM & Estimativa & Desv.Pad. & EQM \\
\hline \multirow{3}{*}{500} & $\hat{\beta}_{0}$ & 0,85079 & 0,69524 & 0,50562 & 1,02730 & 0,84490 & 0,71459 \\
& $\hat{\beta}_{1}$ & 1,53040 & 0,35641 & 0,40838 & 0,98609 & 0,43274 & 0,18746 \\
& $\hat{\beta}_{2}$ & 0,90782 & 0,04120 & 0,01020 & 1,00180 & 0,04960 & 0,00246 \\
\hline \multirow{3}{*}{1000} & $\hat{\beta}_{0}$ & 1,44650 & 0,62075 & 0,58470 & 0,99636 & 0,75628 & 0,57198 \\
& $\hat{\beta}_{1}$ & 1,22330 & 0,30231 & 0,14125 & 1,00420 & 0,36359 & 0,13222 \\
& $\hat{\beta}_{2}$ & 0,93918 & 0,03350 & 0,00482 & 0,99918 & 0,03974 & 0,00158 \\
\hline \multirow{3}{*}{2000} & $\hat{\beta}_{0}$ & 1,20320 & 0,41766 & 0,21573 & 1,02260 & 0,51281 & 0,26349 \\
& $\hat{\beta}_{1}$ & 1,36920 & 0,20749 & 0,17934 & 0,99109 & 0,25308 & 0,06413 \\
& $\hat{\beta}_{2}$ & 0,92205 & 0,02341 & 0,00662 & 1,00070 & 0,02826 & 0,00080 \\
\hline \multirow{3}{*}{3000} & $\hat{\beta}_{0}$ & 1,19520 & 0,41306 & 0,20873 & 0,99967 & 0,51422 & 0,26442 \\
& $\hat{\beta}_{1}$ & 1,39980 & 0,19895 & 0,19943 & 0,99847 & 0,24587 & 0,06046 \\
& $\hat{\beta}_{2}$ & 0,91679 & 0,02190 & 0,00740 & 1,00050 & 0,02680 & 0,00072 \\
\hline \multirow{3}{*}{4000} & $\hat{\beta}_{0}$ & 1,24270 & 0,35233 & 0,18306 & 1,00540 & 0,43683 & 0,19085 \\
& $\hat{\beta}_{1}$ & 1,35880 & 0,16847 & 0,15712 & 0,99614 & 0,20731 & 0,04299 \\
& $\hat{\beta}_{2}$ & 0,92216 & 0,01840 & 0,00640 & 1,00050 & 0,02243 & 0,00050 \\
\hline
\end{tabular}

Como pode ser observado na tabela os estimadores "naive" dos parâmetros de um modelo quadrático não se aproximam dos valores adotados na presente simulação, no gráfico poderemos acompanhar essa situação. 


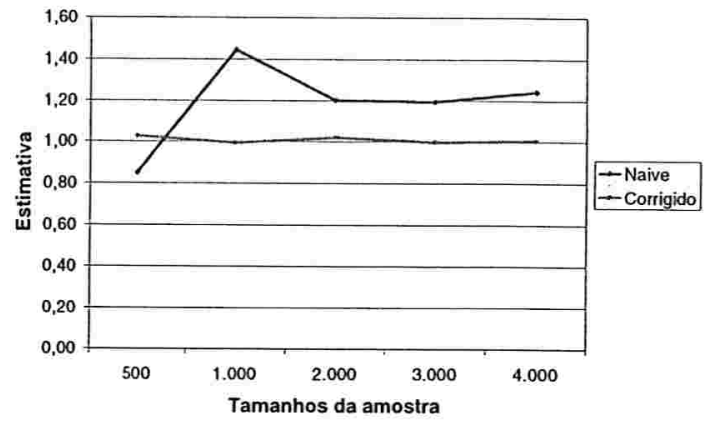

(a) Estimativa para $\beta_{0}$

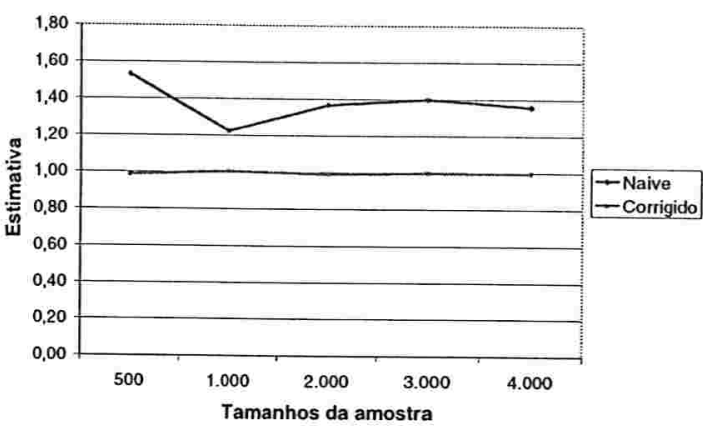

(b) Estimativa para $\beta_{1}$

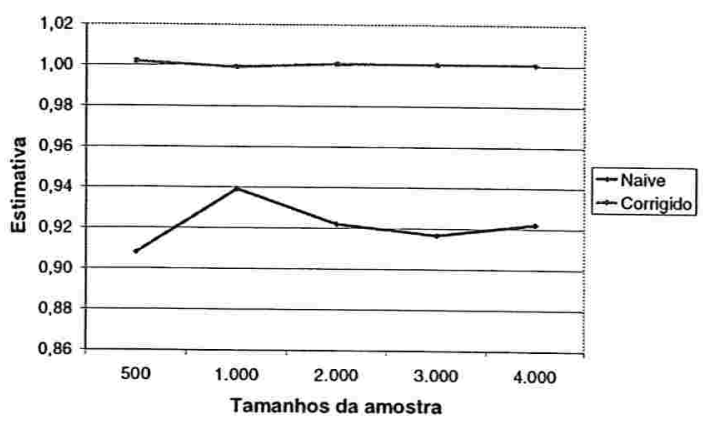

(c) Estimativa para $\beta_{2}$

Fig. 2.12. Comportamento das estimativas "naive" e "escore corrigido" segundo o tamanho da amostra

Como pode ser observado tanto na tabela quanto nos gráficos, que os estimadores pelo método de escore corrigido dos parâmetros de um modelo quadrático variam em torno dos valores fixados na presente simulação, enquanto os estimadores pelo método "naive" não conseguem aproximar-se a estes valores fixados. 
a) Um caso prático

Como exemplo ilustrativo tomamos os dados do exemplo 4 em Galea-Rojas, de Castilho, Bolfarine e de Castro (2003), onde foi considerada o problema da presencia de partículas de grossas de ouro na amostra num depósito de barris. Para avaliar esta situação considerou-se uma tela de ensaio contendo 501 amostras preparadas baixo dois tipos de análise químicas, em cada amostra foi considerada uma variabilidade particular para $\sigma_{e}^{2}$ e $\sigma_{u}^{2}$, os dados do exemplo 4 são apresentados no anexo.

\section{Resultados}

O gráfico abaixo apresenta a distribuição dos dados a serem considerados na presente metodologia.

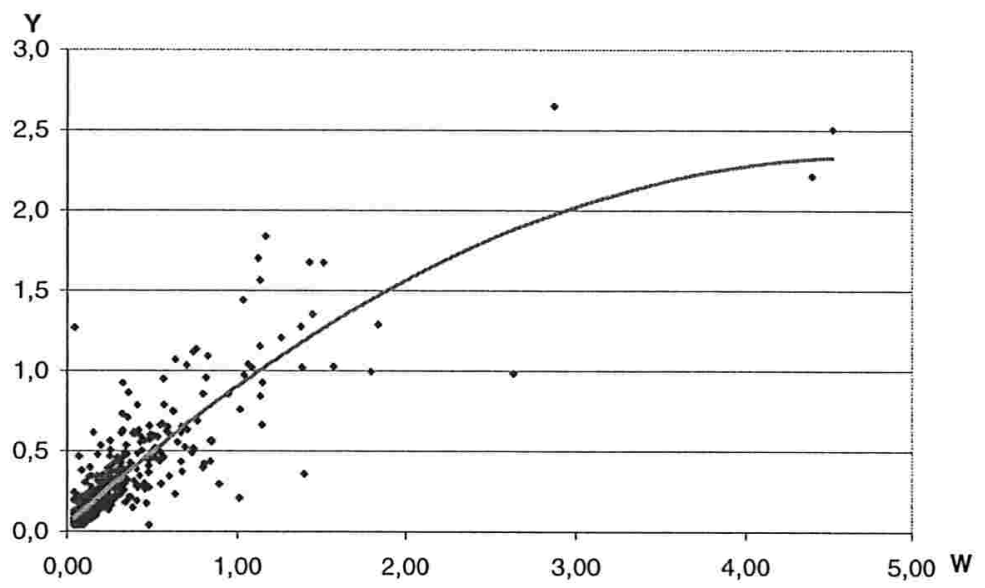

Fig. 2.13. Gráfico de dispersão dos dados

Após implementar a metodologia no linguagem Ox (Doornik,1998) obtemos a tabela a seguir apresentando as estimativas, desvios padrões, assim como os testes de hipóteses para cada parâmetro em estudo $\left(\beta_{0}, \beta_{1}\right.$ e $\left.\beta_{2}\right)$ para um modelo quadrático. 
Tabela 2.9. Estimativas e testes de hipóteses para os parâmetros de um modelo quadrático

\begin{tabular}{|c|c|c|c|c|c|c|c|c|}
\hline Parâmetro & Estimativa & Desv.Pad. & Raz.Var. & $\mathrm{p}$ & Wald & $\mathrm{p}$ & escore & $\mathrm{p}$ \\
\hline$\beta_{0}$ & 0,47412 & 0,07409 & 3884,9 & 0 & 1326,7 & 0 & 36863,0 & 0 \\
$\beta_{1}$ & $-1,67880$ & 0,36417 & 44072,0 & 0 & 769,1 & 0 & 25290,0 & 0 \\
$\beta_{2}$ & 0,63764 & 0,13876 & 883,3 & 0 & 56,5 & 0 & 34368,0 & 0 \\
\hline
\end{tabular}

Onde p é o nível descritivo do teste.

Observamos na tabela acima que as estimativas $\left(\beta_{0}, \beta_{1}\right.$ e $\left.\beta_{2}\right)$ existem no modelo, de acordo com isto o modelo quadrático se esta ajustando melhor aos dados propostos neste exemplo, para fazer uma comparação consideramos um modelo linear com a finalidade de observar se os dados se ajustam melhor a um modelo linear que a um quadrático. Para isto apresentamos o gráfico abaixo

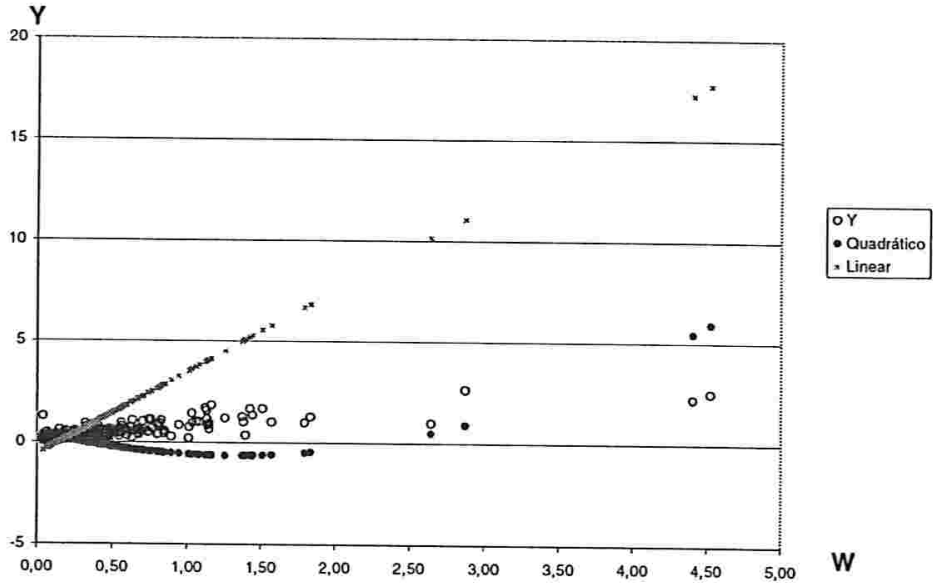

Fig. 2.14. Gráfico de dois modelos propostos, Linear e Quadrático

Segundo o gráfico acima pode-se ver que os dados se ajustam melhor a um modelo quadrático que a um linear. 


\section{CAPÍTULO}

\section{Modelo Multiplicativo}

\subsection{Introdução}

Nesta seção estudaremos os modelos polinomiais com erros nas variáveis e com erros de medição multiplicativo na covariável. Este modelo é definido pelas equações

$$
\begin{gathered}
Y_{i}=\sum_{j=1}^{p} \beta_{j} X_{i}^{j}+e_{i}, \\
W_{i}=X_{i} u_{i}, i=1, \ldots, n .
\end{gathered}
$$

As variáveis no modelo acima têm o mesmo significado que as variáveis definidas no Capítulo 2, para o caso aditivo. A expressão (3.2) constitui o chamado modelo multiplicativo, que foi pouco estudado na literatura, de modo que julgamos relevante estudá-lo segundo o enfoque de Nakamura (1990) e Bolfarine e Gimenez (1997).

Na seção 3.2 será estudada a estimação dos parâmetros, as variâncias assintóticas dos estimadores e os testes de hipóteses de interesse no caso em que $\sigma_{u}^{2}$ é conhecida. Na seção 3.3 será estudada a estimação dos parâmetros, as variâncias assintóticas dos estimadores e os testes de hipóteses de interesse no caso em que $\lambda=\frac{\sigma_{e}^{2}}{\sigma_{u}^{2}}$ conhecida. Na seção 3.4 enfocamos um modelo em que $\sigma_{e}^{2}$ e $\sigma_{u}^{2}$ são heterogêneas e conhecidas. 


\subsection{Caso $1: \sigma_{u}^{2}$ conhecida}

\subsubsection{Estimação dos parâmetros}

Em modelos funcionais se trabalha com a suposição seguinte:

$$
\left(\begin{array}{c}
e_{i} \\
u_{i}
\end{array}\right) \sim N_{2}\left[\left(\begin{array}{l}
0 \\
1
\end{array}\right),\left(\begin{array}{cc}
\sigma_{e}^{2} & 0 \\
0 & \sigma_{u}^{2}
\end{array}\right)\right] \text {, independentes, } i=1, \ldots, n,
$$

enquanto em modelos estruturais uma suposição usualmente adotada é definida por

$$
\left(\begin{array}{c}
X_{i} \\
e_{i} \\
u_{i}
\end{array}\right) \sim N_{3}\left[\left(\begin{array}{c}
\mu_{X} \\
0 \\
1
\end{array}\right),\left(\begin{array}{ccc}
\sigma_{X}^{2} & 0 & 0 \\
0 & \sigma_{e}^{2} & 0 \\
0 & 0 & \sigma_{u}^{2}
\end{array}\right)\right] \text { independentes, } i=1, \ldots, n .
$$

Para qualquer um das suposições mencionadas acima a função log-verossimilhança "naive" (ingênua) é definida como

$$
\ell\left(\beta, \sigma_{u}^{2} ; \mathrm{W}, \mathrm{Y}\right)=-\frac{n}{2} \log (2 \pi)-\frac{n}{2} \log \left(\sigma_{e}^{2}\right)-\frac{1}{2 \sigma_{e}^{2}} \sum_{i=1}^{n}\left(Y_{i}-\sum_{j=0}^{p} \beta_{j} W_{i}^{j}\right)^{2} .
$$

Para estimar os parâmetros a partir desta função log-verossimilhança recorremos aos passos das seções 2.2.1 (a) e (b) a fim de encontrar a função verossimilhança corrigida e também a função escore corrigido.

Para estabelecer a relação apresentada no capítulo 2 , entre a variável auxiliar $t_{i,(s)}$ e a variável observável $W_{i}$ consideramos o seguinte

$$
\begin{gathered}
W_{i}^{s}=X_{i}^{s} u_{i}^{s}, \\
E\left(W_{i}^{s}\right)=E\left(X_{i}^{s} u_{i}^{s}\right),
\end{gathered}
$$

No caso funcional temos que $X_{i}$ é fixo, de modo que

$$
E\left(W_{i}^{s}\right)=X_{i}^{s} E\left(u_{i}^{s}\right)
$$

$\mathrm{e}$ 


$$
X_{i}^{s}=\frac{E\left(W_{i}^{s}\right)}{E\left(u_{i}^{s}\right)},
$$

enquanto no caso estrutural temos que umas das suposições possíveis é que $X_{i}$ e $u_{i}$ sejam independentes, de forma que

$$
E\left(X_{i}^{s}\right)=\frac{E\left(W_{i}^{s}\right)}{E\left(u_{i}^{s}\right)}, \quad i=1, \ldots, n .
$$

Se nas expressões (3.6) e (3.7) consideramos as mudanças de variáveis $X_{i}^{s}$ por $t_{i,(s)}$ e $E\left(W_{i}^{s}\right)$ por $W_{i}^{s}$ para o caso funcional, e $E\left(X_{i}^{s}\right)$ por $t_{i,(s)}$ e $E\left(W_{i}^{s}\right)$ por $W_{i}^{s}$ para o caso estrutural, temos que ambas as expressões podem ser substituídas por,

$$
t_{i,(s)}=\frac{W_{i}^{s}}{E\left(u_{i}^{s}\right)}, \quad s=0,1, \ldots, p, \mathrm{e} i=1, \ldots, n .
$$

Para obter os momentos da variável $u_{i}$ utilizamos a proposição seguinte:

Proposição 2 Se $H_{i} \sim N\left(1, \sigma_{h}^{2}\right)$, a função geradora de momentos da variável $H_{i} \quad$ é $\quad M_{H_{i}}(t)=\exp \left(t+\frac{1}{2} \sigma_{h}^{2} t^{2}\right)$, valendo a seguinte relação:

$$
\frac{\partial^{(j+1)}}{\partial t^{(j+1)}} M_{H_{i}}(t)=\left(1+t \sigma_{h}^{2}\right) \frac{\partial^{(j)}}{\partial t^{(j)}} M_{H_{i}}(t)-j \sigma_{h}^{2} \frac{\partial^{(j-1)}}{\partial t^{(j-1)}} M_{H_{i}}(t)
$$

para $j \geq 2$, onde $\partial t^{(\mathrm{q})}$ denota a q-ésima derivada parcial em relação a $t$.

Usando a relação da proposição 2 para obter o q-ésimo momento da variável $H_{i}$, temos

$$
E\left(H_{i}^{q}\right)=\frac{\partial^{(q)}}{\partial t^{(q)}} M_{H_{i}}(0)=E\left(H^{q-1}\right)+(q-1) \sigma_{h}^{2} E\left(H^{q-2}\right) .
$$

Então, para a variável $u_{i}$ temos a relação seguinte:

$$
E\left(u_{i}^{s}\right)=E\left(u_{i}^{s-1}\right)+(s-1) \sigma_{u}^{2} E\left(u_{i}^{s-2}\right), \quad s \geq 2 .
$$

Seguindo os procedimentos para a obtenção do logaritmo da função verossimilhança corrigida no Capítulo 2, temos que o logaritmo da função de verossimilhança corrigida para o modelo multiplicativo é dado como em (2.9), isto é, 


$$
\begin{aligned}
\ell^{*}\left(\beta, \sigma_{e}^{2} ; \mathrm{Y}, \mathrm{W}\right)= & -\frac{n}{2} \log (2 \pi)-\frac{n}{2} \log \left(\sigma_{e}^{2}\right)-\frac{1}{2 \sigma_{e}^{2}}\left\{\sum _ { i = 1 } ^ { n } \left[Y_{i}^{2}+\sum_{j=0}^{p} \beta_{j}^{2} t_{i,(2 j)}\right.\right. \\
& \left.\left.-2 Y_{i} \sum_{j=0}^{p} \beta_{j} t_{i,(j)}+2 \sum_{\substack{j=0 \\
s<j}}^{p} \sum_{s=0}^{p} \beta_{j} \beta_{s} t_{i,(j+s)}\right]\right\}
\end{aligned}
$$

onde $t_{i,(s)}$ tem a forma (3.8).

Considerando o logaritmo da função verossimilhança corrigida apresentada acima e derivando com relação a cada parâmetro em $\left(\beta, \sigma_{e}^{2}\right)$, temos como na seção 2.2.1:

$$
\begin{aligned}
& \left(\begin{array}{cccc}
n & \sum_{i=1}^{n} t_{i,(1)} & \cdots & \sum_{i=1}^{n} t_{i,(p)} \\
\sum_{i=1}^{n} t_{i,(1)} & \sum_{i=1}^{n} t_{i,(2)} & \cdots & \sum_{i=1}^{n} t_{i,(p+1)} \\
\vdots & \vdots & \cdots & \vdots \\
\sum_{i=1}^{n} t_{i,(p)} & \sum_{i=1}^{n} t_{i,(p+1)} & \cdots & \sum_{i=1}^{n} t_{i,(2 p)}
\end{array}\right)\left(\begin{array}{c}
\hat{\beta}_{0} \\
\hat{\beta}_{1} \\
\vdots \\
\hat{\beta}_{p}
\end{array}\right)=\left(\begin{array}{c}
\sum_{i=1}^{n} Y_{i} \\
\sum_{i=1}^{n} Y_{i} t_{i,(1)} \\
\vdots \\
\sum_{i=1}^{n} Y_{i} t_{i,(p)}
\end{array}\right), \\
& \hat{\sigma}_{e}^{2}=\frac{1}{n} \sum_{i=1}^{n}\left[Y_{i}^{2}+\sum_{j=0}^{p} \hat{\beta}_{j}^{2} t_{i,(2 j)}-2 Y_{i} \sum_{j=0}^{p} \hat{\beta}_{j} t_{i,(j)}+2 \sum_{j=0}^{p} \sum_{s=0}^{p} \hat{\beta}_{j} \hat{\beta}_{s} t_{i,(j+s)}\right] .
\end{aligned}
$$

A partir destas expressões e da expressão (3.8) podemos obter os estimadores dos parâmetros. 


\subsubsection{Exemplos}

\section{Polinômio de primeiro grau}

Considerando a expressão (3.10) e (3.11), para $\mathrm{p}=1$, temos

$$
\begin{gathered}
\left(\begin{array}{cc}
n & \sum_{i=1}^{n} t_{i,(1)} \\
\sum_{i=1}^{n} t_{i,(1)} & \sum_{i=1}^{n} t_{i,(2)}
\end{array}\right)\left(\begin{array}{l}
\hat{\beta}_{0} \\
\hat{\beta}_{1}
\end{array}\right)=\left(\begin{array}{c}
\sum_{i=1}^{n} Y_{i} \\
\sum_{i=1}^{n} Y_{i} t_{i,(1)}
\end{array}\right), \\
\hat{\sigma}_{e}^{2}=\frac{1}{n} \sum_{i=1}^{n}\left[Y_{i}^{2}+\hat{\beta}_{0}^{2}+\hat{\beta}_{1}^{2} t_{i,(2)}-2 Y_{i} \hat{\beta}_{0}-2 Y_{i} \hat{\beta}_{1} t_{i,(1)}+2 \hat{\beta}_{0} \hat{\beta}_{1} t_{i,(1)}\right] .
\end{gathered}
$$

Levando em conta que $t_{i,(1)}=W_{i}$ e $t_{i,(2)}=\frac{W_{i}^{2}}{\sigma_{u}^{2}+1}$, temos os seguintes estimadores:

$$
\begin{gathered}
\hat{\beta}_{0}=\bar{Y}-\bar{W} \hat{\beta}_{1}, \\
\hat{\beta}_{1}=\frac{\left(\sigma_{u}^{2}+1\right) S_{Y W}}{S_{W W}-n \bar{W}^{2} \sigma_{u}^{2}},
\end{gathered}
$$

onde $S_{W W}$ e $S_{Y W}$ são a variância amostral de W e a covariância amostral de W e $\mathrm{Y}$, respectivamente que foram definidas no Capítulo 2 , seção exemplos polinômio de 1 grau,

O estimador de $\beta_{1}$ acima foi obtido por Hwang, J. (1986) e Nakamura (1990), ver também Gasco, L. et al. (2002)

$$
\hat{\sigma}_{e}^{2}=\frac{1}{n} \sum_{i=1}^{n}\left(Y_{i}-\hat{\beta}_{0}-\hat{\beta}_{1} W_{i}-\hat{\beta}_{2} W_{i}^{2}\right)^{2}-\frac{\hat{\beta}_{1}^{2} \sigma_{u}^{2}}{\left(\sigma_{u}^{2}+1\right)}\left(S_{W W}+\bar{W}^{2}\right) .
$$




\section{Polinômio de segundo grau}

Considerando as expressões (3.10) e (3.11), para $\mathrm{p}=2$, temos

$$
\begin{aligned}
& \left(\begin{array}{ccc}
n & \sum_{i=1}^{n} t_{i,(1)} & \sum_{i=1}^{n} t_{i,(2)} \\
\sum_{i=1}^{n} t_{i,(1)} & \sum_{i=1}^{n} t_{i,(2)} & \sum_{i=1}^{n} t_{i,(3)} \\
\sum_{i=1}^{n} t_{i,(2)} & \sum_{i=1}^{n} t_{i,(3)} & \sum_{i=1}^{n} t_{i,(4)}
\end{array}\right)\left(\begin{array}{l}
\hat{\beta}_{0} \\
\hat{\beta}_{1} \\
\hat{\beta}_{2}
\end{array}\right)=\left(\begin{array}{c}
\sum_{i=1}^{n} Y_{i} \\
\sum_{i=1}^{n} Y_{i} t_{i,(1)} \\
\sum_{i=1}^{n} Y_{i} t_{i,(2)}
\end{array}\right), \\
& \hat{\sigma}_{e}^{2}=\frac{1}{n} \sum_{i=1}^{n}\left[Y_{i}^{2}+\hat{\beta}_{0}^{2}+\hat{\beta}_{1}^{2} t_{i,(2)}+\hat{\beta}_{2}^{2} t_{i,(4)}-2 Y_{i} \hat{\beta}_{0}-2 Y_{i} \hat{\beta}_{1} t_{i,(1)}\right. \\
& \left.-2 Y_{i} \hat{\beta}_{2} t_{i,(2)}+2 \hat{\beta}_{0} \hat{\beta}_{1} t_{i,(1)}+2 \hat{\beta}_{0} \hat{\beta}_{2} t_{i,(2)}+2 \hat{\beta}_{1} \hat{\beta}_{2} t_{i,(3)}\right] .
\end{aligned}
$$

Como

$$
t_{i,(1)}=\frac{W_{i}}{E\left(u_{i}\right)}, \quad t_{i,(2)}=\frac{W_{i}^{2}}{E\left(u_{i}^{2}\right)}, \quad t_{i,(3)}=\frac{W_{i}^{3}}{E\left(u_{i}^{3}\right)} \quad \text { e } \quad t_{i,(4)}=\frac{W_{i}^{4}}{E\left(u_{i}^{4}\right)},
$$

usando a proposição $2 \mathrm{com}, E\left(u_{i}\right)=1, \quad E\left(u_{i}^{2}\right)=1+\sigma_{u}^{2}, \quad E\left(u_{i}^{3}\right)=1+3 \sigma_{u}^{2} \quad$ e $E\left(u_{i}^{4}\right)=1+6 \sigma_{u}^{2}+3 \sigma_{u}^{4}$. Por outro lado, levando em conta

$$
\begin{aligned}
\sum_{i=1}^{n} W_{i}^{2} & =n\left(S_{W W}+\bar{W}^{2}\right) \\
\sum_{i=1}^{n} W_{i}^{3} & =n\left[S_{W W^{2}}+\bar{W}\left(S_{W W}+\bar{W}^{2}\right)\right] \\
\sum_{i=1}^{n} W_{i}^{4} & =n\left[S_{W^{2} W^{2}}+\left(S_{W W}+\bar{W}^{2}\right)^{2}\right] \\
\sum_{i=1}^{n} Y_{i} W_{i} & =n\left(S_{Y W}+\overline{Y W}\right), \\
\sum_{i=1}^{n} Y_{i} W_{i}^{2} & =n\left[S_{Y W^{2}}+\bar{Y}\left(S_{W W}+\bar{W}^{2}\right)\right.
\end{aligned}
$$

sendo $S_{W W}$ a variância amostral da variável W, $S_{W W^{2}}$ a covariâncias amostral das variáveis $W$ e $W^{2}, S_{W^{2} W^{2}}$ a covariâncias amostral das variáveis $W^{2}$ e $W^{2}, S_{Y W}$ a 
covariâncias amostral das variáveis $Y$ e $W$ e $S_{Y W^{2}}$ a covariâncias amostral das variáveis $Y$ e $W^{2}$, os estimadores podem ser escritos como

$$
\begin{aligned}
& \hat{\beta}_{0}=\bar{Y}-\bar{W} \hat{\beta}_{1}-\frac{1}{\sigma_{u}^{2}}\left(S_{W W}+\bar{W}^{2}\right) \hat{\beta}_{2}, \\
& \hat{\beta}_{1}=\left[\frac{\left(\sigma_{u}^{2}+1\right) S_{Y W}}{S_{W W}-\bar{W}^{2} \sigma_{u}^{2}}\right]-B \hat{\beta}_{2} \\
& \hat{\beta}_{2}=\frac{S_{Y W^{2}}}{A+B}-\left[\frac{\left(\sigma_{u}^{2}+1\right) S_{Y W}}{S_{W W}-\bar{W}^{2} \sigma_{u}^{2}}\right]\left[\frac{B}{A+B}\right],
\end{aligned}
$$

onde

$$
\begin{aligned}
& A=\frac{\left(\sigma_{u}^{2}+1\right) S_{W W^{2}}-2 \sigma_{u}^{2}\left(S_{W W}+\bar{W}^{2}\right)^{2}}{3 \sigma_{u}^{4}+6 \sigma_{u}^{2}+1} \\
& B=\frac{\left(\sigma_{u}^{2}+1\right) S_{W W^{2}}-2 \bar{W} \sigma_{u}^{2}\left(S_{W W}+\bar{W}^{2}\right)}{3 \sigma_{u}^{2}+1}
\end{aligned}
$$

sendo o estimador de $\sigma_{e}^{2}$ dado por

$$
\begin{aligned}
\hat{\sigma}_{e}^{2}= & \frac{1}{n} \sum_{i=1}^{n}\left(Y_{i}-\hat{\beta}_{0}-\hat{\beta}_{1} W_{i}-\hat{\beta}_{2} W_{i}^{2}\right)^{2}-\frac{\hat{\beta}_{1} \sigma_{u}^{2}}{\sigma_{u}^{2}+1}\left(S_{W W}+\bar{W}^{2}\right) \\
& -\frac{3 \hat{\beta}_{2}^{2} \sigma_{u}^{2}\left(\sigma_{u}^{2}+2\right)}{3 \sigma_{u}^{4}+6 \sigma_{u}^{2}+1}\left[S_{W^{2} W^{2}}+\left(S_{W W}+\bar{W}^{2}\right)^{2}\right]+\frac{2 \hat{\beta}_{2} \sigma_{u}^{2}}{\sigma_{u}^{2}+1}\left[S_{Y W^{2}}+\bar{Y}\left(S_{W W}+\bar{W}^{2}\right)\right] \\
& -\frac{2 \hat{\beta}_{0} \hat{\beta}_{2} \sigma_{u}^{2}}{\sigma_{u}^{2}+1}\left(S_{W W}+\bar{W}^{2}\right)+\frac{4 \hat{\beta}_{1} \hat{\beta}_{2} \sigma_{u}^{2}}{3 \sigma_{u}^{2}+1}\left[S_{W W^{2}}+\bar{W}\left(S_{W W}+\bar{W}^{2}\right)\right] .
\end{aligned}
$$

\subsubsection{Obtenção da matriz de covariâncias assintóticas}

O calculo da matriz de covariâncias assintóticas é similar ao modo apresentado na seção 2.2 .2 , sendo

$$
£^{*}=\frac{1}{n}\left(\mathbf{I}^{*}(\hat{\theta} ; \mathrm{W}, \mathrm{Y})\right)^{-1} \mathrm{~S}^{*}(\hat{\theta} ; \mathrm{W}, \mathrm{Y})\left(\mathbf{I}^{*}(\hat{\theta} ; \mathrm{W}, \mathrm{Y})\right)^{-1}
$$

onde, 


$$
\mathbf{I}^{*}(\hat{\beta} ; \mathrm{W}, \mathrm{Y})=-\frac{1}{n} \sum_{i=1}^{n} \frac{\partial}{\partial \beta} U_{i}^{*}(\hat{\beta} ; \mathrm{W}, \mathrm{Y})
$$

e

$$
\mathrm{S}^{*}(\hat{\beta} ; \mathrm{W}, \mathrm{Y})=\frac{1}{n} \sum_{i=1}^{n} \mathrm{U}_{i}^{*}(\hat{\beta} ; \mathrm{W}, \mathrm{Y}) \mathbf{U}_{i}^{*}(\hat{\beta} ; \mathrm{W}, \mathrm{Y})^{t}
$$

concluindo que

$$
\sqrt{n}(\hat{\beta}-\beta) \stackrel{D}{\longrightarrow} N_{p+1}\left(0, £^{*}\right)
$$

\subsubsection{Teste de hipóteses para $\beta$}

Nesta seção consideramos os mesmos testes de hipóteses apresentados na seção 2.2.3, sendo

$$
\begin{aligned}
U^{*}(\theta)=\left(\begin{array}{c}
U^{*}\left(\beta_{0}\right) \\
U^{*}\left(\beta_{1}\right) \\
\vdots \\
U^{*}\left(\beta_{p}\right) \\
U^{*}\left(\sigma_{e}^{2}\right)
\end{array}\right)=\left(\begin{array}{c}
U^{*}(\beta) \\
U^{*}\left(\sigma_{e}^{2}\right)
\end{array}\right), \\
I^{*}(\theta)=\left(\begin{array}{ccc}
-\frac{\partial}{\partial \beta_{0}} U^{*}\left(\beta_{1}\right)-\frac{\partial}{\partial \beta_{1}} U^{*}\left(\beta_{1}\right) \cdots-\frac{\partial}{\partial \beta_{p}} U^{*}\left(\beta_{1}\right)-\frac{\partial}{\partial \sigma_{e}^{2}} U^{*}\left(\beta_{1}\right) \\
\vdots \\
\vdots \\
-\frac{\partial}{\partial \beta_{0}} U^{*}\left(\beta_{p}\right)-\frac{\partial}{\partial \beta_{1}} U^{*}\left(\beta_{p}\right) \cdots-\frac{\partial}{\partial \beta_{p}} U^{*}\left(\beta_{p}\right)-\frac{\partial}{\partial \sigma_{e}^{2}} U^{*}\left(\beta_{p}\right) \\
-\frac{\partial}{\partial \beta_{0}} U^{*}\left(\sigma_{e}^{2}\right)-\frac{\partial}{\partial \beta_{1}} U^{*}\left(\sigma_{e}^{2}\right) \cdots-\frac{\partial}{\partial \beta_{p}} U^{*}\left(\sigma_{e}^{2}\right)-\frac{\partial}{\partial \sigma_{e}^{2}} U^{*}\left(\sigma_{e}^{2}\right)
\end{array}\right) \\
=\left(\begin{array}{cc}
-\frac{\partial}{\partial \beta} U^{*}(\beta) & -\frac{\partial}{\partial \sigma_{e}^{2}} U^{*}(\beta) \\
-\frac{\partial}{\partial \beta} U^{*}\left(\sigma_{e}^{2}\right)-\frac{\partial}{\partial \sigma_{e}^{2}} U^{*}\left(\sigma_{e}^{2}\right)
\end{array}\right) \cdot
\end{aligned}
$$

A partir dessas expressões podemos considerar que $\bar{\Lambda}_{n}(\theta)=\frac{1}{n} E\left[I^{*}(\theta)\right]$, onde $\theta=\left(\beta^{t}, \sigma_{e}^{2}\right)^{t}$ e $\bar{\Gamma}_{n}(\theta)=\frac{1}{n} \sum_{i=1}^{n} E\left[U_{i}^{*}(\theta) U_{i}^{*}(\theta)^{t}\right]$, quando $\mathrm{n}$ é grande as matrices acima apresentadas, convergem para as matrizes positivas definidas $\Lambda(\theta)$ e $\Gamma(\theta)$. Como nosso interesse é testar a hipóteses nula $H_{0}: \beta=\beta^{0}$ na presença do parâmetro "nuisance" $\sigma_{e}^{2}$, as matrizes particionadas segundo as dimensões de $\sigma_{e}^{2}$ e $\beta$ são 


$$
\Lambda(\theta)=\left(\begin{array}{cc}
\Lambda_{\beta \beta}(\theta) & \Lambda_{\beta \sigma_{e}^{2}}(\theta) \\
\Lambda_{\sigma_{e}^{2} \beta}(\theta) & \Lambda_{\sigma_{e}^{2} \sigma_{e}^{2}}(\theta)
\end{array}\right) \quad \Gamma(\theta)=\left(\begin{array}{cc}
\Gamma_{\beta \beta}(\theta) & \Gamma_{\beta \sigma_{e}^{2}}(\theta) \\
\Gamma_{\sigma_{e}^{2} \beta}(\theta) & \Gamma_{\sigma_{e}^{2} \sigma_{e}^{2}}(\theta)
\end{array}\right)
$$

onde as matrizes $\Lambda_{\beta \beta}(\theta)$ ou $\Gamma_{\beta \beta}(\theta)$ são de dimensão $(p+1) \times(p+1)$, os vetores $\Lambda_{\beta \sigma_{e}^{2}}(\theta)$ ou $\Gamma_{\beta \sigma_{e}^{2}}(\theta)$ são de dimensão $1 \times(p+1)$ e o escalar $\Lambda_{\sigma_{e}^{2} \sigma_{e}^{2}}(\theta)$ ou $\Gamma_{\sigma_{e}^{2} \sigma_{e}^{2}}(\theta)$ são de dimensão $1 \times 1$.

Sejam $\hat{\theta}=\left(\hat{\beta}^{t}, \hat{\sigma}_{e}^{2}\right)^{t}$ e $\hat{\theta}_{0}=\left(\hat{\beta}_{0}^{t}, \hat{\sigma}_{e}^{2}\right)^{t}$ estimadores consistentes de $\theta$, satisfazendo $U^{*}(\hat{\theta})=0$ e $U^{*}(\hat{\beta})=0$, ou seja, $\hat{\theta}$ e $\hat{\theta}_{0}$ são estimadores corrigidos irrestritos e restritos a $H_{0}$, respectivamente. Os testes de Wald e Escore que podem ser aplicados são

$$
\begin{gathered}
W=n\left(\hat{\beta}-\beta^{0}\right)^{t} \hat{\Lambda}_{\beta \beta . \sigma_{e}^{2}}\left(\hat{\theta}_{0}\right)\left(\hat{\beta}-\beta^{0}\right), \\
Q=\frac{1}{n} U_{\beta}^{*}\left(\hat{\theta}_{0}\right)^{t} \hat{\Lambda}_{\beta \beta . \sigma_{e}^{2}}^{-1}\left(\hat{\theta}_{0}\right) U_{\beta}^{*}\left(\hat{\theta}_{0}\right), \\
W_{c}=n\left(\hat{\beta}-\beta^{0}\right)^{t} \hat{\Omega}_{\beta \beta}^{-1}\left(\hat{\theta}_{0}\right)\left(\hat{\beta}-\beta^{0}\right),
\end{gathered}
$$

sendo

$$
\hat{\Lambda}_{\beta \beta . \sigma_{e}^{2}}\left(\hat{\theta}_{0}\right)=\hat{\Lambda}_{\beta \beta}\left(\hat{\theta}_{0}\right)-\hat{\Lambda}_{\sigma_{e}^{2} \sigma_{e}^{2}}^{-1}\left(\hat{\theta}_{0}\right) \hat{\Lambda}_{\beta \sigma_{e}^{2}}\left(\hat{\theta}_{0}\right)
$$

e

$$
\hat{\Omega}_{\beta \beta}\left(\hat{\theta}_{0}\right)=\left(\hat{\Lambda}^{-1}\left(\hat{\theta}_{0}\right) \hat{\Gamma}\left(\hat{\theta}_{0}\right) \hat{\Lambda}^{-1}\left(\hat{\theta}_{0}\right)\right)_{\beta \beta}
$$

assim também

$$
Q_{c}=\frac{1}{n} U_{\beta}^{*}\left(\hat{\theta}_{0}\right)^{t} \hat{\Lambda}_{\beta \beta . \sigma_{e}^{2}}^{-1}\left(\hat{\theta}_{0}\right) \hat{\Omega}_{\beta \beta}^{-1}\left(\hat{\theta}_{0}\right) \hat{\Lambda}_{\beta \beta . \sigma_{e}^{2}}^{-1}\left(\hat{\theta}_{0}\right) U_{\beta}^{*}\left(\hat{\theta}_{0}\right)
$$

Quando a função escore corrigido corresponde a gradiente do logaritmo da função de verossimilhança corrigida $\ell^{*}(\theta)$, isto é,

$$
U^{*}(\theta)=\frac{\partial \ell^{*}(\theta)}{\partial \theta}
$$

é possível definir um teste de razão de verossimilhança corrigida. A estatística de razão de verossimilhança corrigida é definida

$$
L=2 \ell^{*}(\hat{\theta})-\ell^{*}\left(\hat{\theta}_{0}\right) .
$$




\subsubsection{Aplicação numérica}

Para a aplicação da metodologia apresentada consideramos duas formas de aplicação, a primeira consistente em uma simulação de dados e uma segunda com dados reais.

\section{a) Um estudo de simulação}

Para esta situação consideramos um modelo funcional em um polinômio de grau dois. Efetuamos 1.000 replicações para estudar a convergência dos estimadores e os seguintes parâmetros são tomados em consideração

1. Para $n$ especificado, considerar $X_{i} \sim \operatorname{Binomial}(n ; 0,5), \quad u_{i} \sim N(1 ; 0,05) \quad$ e $e_{i} \sim N(0 ; 1)$ para $i=1,2, \ldots, n$.

2. Obtenção dos dados:

$$
\begin{aligned}
W_{i} & =X_{i} u_{i}, \\
Y_{i} & =\beta_{0}+\beta_{1} X_{i}+\beta_{2} X_{i}^{2}+e_{i},
\end{aligned}
$$

sendo $\beta_{0}=\beta_{1}=\beta_{2}=1$.

3. Para os efeitos práticos, deve-se considerar as expressões (3.15)-(3.17), para a estimação dos parâmetros.

\section{Resultados}

Após implementada a metodologia apresentada na linguagem Ox (Doonik, 1998), temos os seguintes resultados experimentais que são apresentados em forma resumida na tabela abaixo. Nesta tabela encontram-se as estimativas de um modelo quadrático, considerando o desvio gerado para cada estimativa, assim como o erro quadrático médio. 
Tabela 3.1. Estimativas segundo os métodos "naive"e "escore corrigido" para diferentes tamanhos de amostra.

\begin{tabular}{|c|c|c|c|c|c|c|c|}
\hline \multirow[t]{2}{*}{$\mathrm{n}$} & \multirow[t]{2}{*}{ Parâmetro } & \multicolumn{3}{|c|}{ Naive } & \multicolumn{3}{|c|}{ Escore corrigido } \\
\hline & & Estimativa & Desv.Pad. & EQM & Estimativa & Desv.Pad. & EQM \\
\hline \multirow{4}{*}{5000} & $\hat{\beta}_{0}$ & 1,36316 & 0,07830 & 0,75294 & 1,01236 & 0,09462 & 0,00719 \\
\hline & $\hat{\beta}_{1}$ & 0,80059 & 0,06058 & 0,43581 & 0,94613 & 0,15071 & 0,10533 \\
\hline & $\hat{\beta}_{2}$ & 0,81168 & 0,07015 & 2,58860 & 1,00112 & 0,13922 & 0,00683 \\
\hline & $\hat{\sigma}_{e}^{2}$ & 3,44628 & - & 2,42016 & 0,24185 & - & 0,00012 \\
\hline \multirow{4}{*}{6000} & $\hat{\beta}_{0}$ & 1,44200 & 0,02171 & 1,05699 & 1,03410 & 0,13267 & 0,01319 \\
\hline & $\hat{\beta}_{1}$ & 0,71663 & 0,09125 & 0,69516 & 0,94472 & 0,19588 & 0,09863 \\
\hline & $\hat{\beta}_{2}$ & 0,81171 & 0,06742 & 1,43404 & 1,03710 & 0,06663 & 0,02035 \\
\hline & $\hat{\sigma}_{e}^{2}$ & 3,46400 & - & 3,42229 & 0,24290 & - & 0,00014 \\
\hline \multirow{4}{*}{7000} & $\hat{\beta}_{0}$ & 1,36496 & 0,06053 & 0,86309 & 0,97612 & 0,07759 & 0,00504 \\
\hline & $\hat{\beta}_{1}$ & 0,76038 & 0,08068 & 0,82293 & 1,00530 & 0,36075 & 0,10549 \\
\hline & $\hat{\beta}_{2}$ & 0,78112 & 0,03427 & 2,30774 & 1,04233 & 0,13811 & 0,01575 \\
\hline & $\hat{\sigma}_{e}^{2}$ & 4,15370 & - & 2,01921 & 0,23735 & - & 0,00042 \\
\hline \multirow{4}{*}{8000} & $\hat{\beta}_{0}$ & 1,41531 & 0,05627 & 0,53438 & 1,04103 & 0,05838 & 0,00301 \\
\hline & $\hat{\beta}_{1}$ & 0,73497 & 0,08848 & 0,85725 & 1,04202 & 0,28083 & 0,04926 \\
\hline & $\hat{\beta}_{2}$ & 0,84314 & 0,03296 & 2,32944 & 1,01476 & 0,07262 & 0,00313 \\
\hline & $\hat{\sigma}_{e}^{2}$ & 3,73979 & - & 2,72934 & 0,24596 & - & 0,00036 \\
\hline \multirow{4}{*}{9000} & $\hat{\beta}_{0}$ & 1,25717 & 0,07063 & 0,91534 & 1,02509 & 0,07522 & 0,01185 \\
\hline & $\hat{\beta}_{1}$ & 0,78058 & 0,10043 & 0,54817 & 0,95670 & 0,34900 & 0,03394 \\
\hline & $\hat{\beta}_{2}$ & 0,82977 & 0,07225 & 1,94595 & 0,99153 & 0,07996 & 0,00549 \\
\hline & $\hat{\sigma}_{e}^{2}$ & 3,32005 & - & 3,48797 & 0,23241 & - & 0,00048 \\
\hline
\end{tabular}

Como se pode observar na tabela acima, as estimativas para os parâmetros $\beta$ obtidas pelo método de "escore corrigido" estão próximos dos valores fixados em nossa simulação, enquanto as estimativas para os parâmetros "naive" não conseguem aproximar-se aos valores fixados em nossa simulação. O resultado da tabela acima são mostrados na figura seguinte 


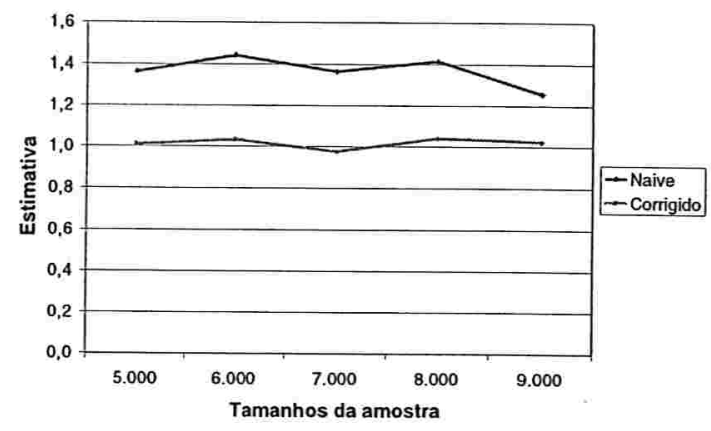

(a) Estimativa para $\beta_{0}$

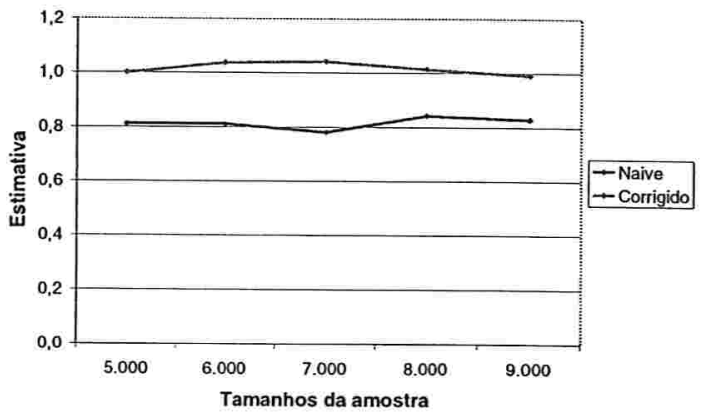

(c) Estimativa para $\beta_{2}$

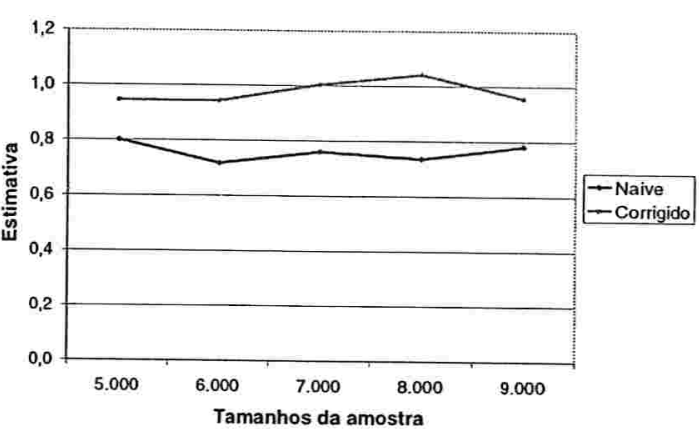

(b) Estimativa para $\beta_{1}$

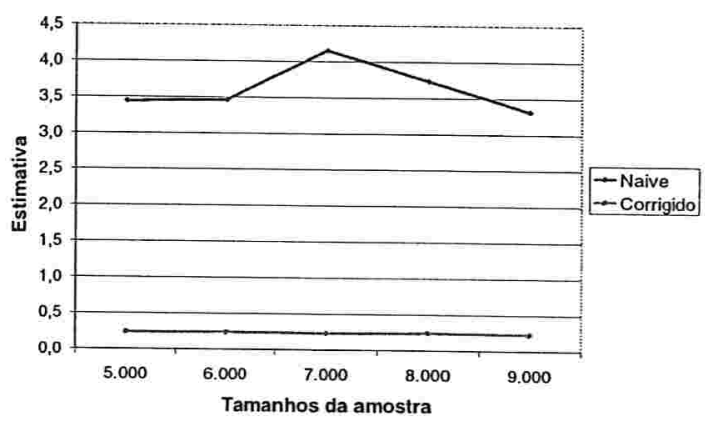

(d) Estimativa para $\sigma_{e}^{2}$

Fig. 3.1. Comportamento das estimativas "naive"e "escore corrigido"segundo o tamanho da amostra

Da figura acima pode-se observar que as estimativas mediante o método "escore corrigido", tem uma maior proximidade com os valores fixados na presente simulação que as estimativas mediante o método "naive".

\section{a) Um caso prático}

Devido a que na literatura estatística não existem trabalhos semelhantes, consideramos os mesmos dados apresentados por Fuller (pag 266) com a finalidade de comparar os 
resultados obtidos com esta metodologia e a metodologia da seção 2.2 , para isto considera-se as variáveis $Y_{1}$ e $W$ que estão no anexo deste trabalho.

\section{Resultados}

Após a implementação da metodologia no linguagem Ox (Doornik, 1998) obtemos a seguinte tabela

Tabela 3.2. Estimativas segundo o método de escore corrigido

\begin{tabular}{|c|c|c||c|c|}
\hline \multirow{2}{*}{ Parâmetro } & \multicolumn{2}{|c||}{ Aditivo } & \multicolumn{2}{c|}{ Multiplicativo } \\
\cline { 2 - 5 } & Estimativa & Desv.Pad. & Estimativa & Desv.Pad. \\
\hline$\beta_{0}$ & $-0,02441$ & 0,11394 & $-0,03824$ & 0,08588 \\
$\beta_{1}$ & 0,54315 & 0,10063 & 0,50089 & 0,07374 \\
$\beta_{2}$ & 1,04690 & 0,24227 & 0,99276 & 0,14833 \\
$\sigma_{e}^{2}$ & 0,08138 & - & 0,11658 & - \\
\hline
\end{tabular}

Observemos na tabela acima que as estimativas obtidas pelo método de escore corrigido tanto para o modelo aditivo quanto para o modelo multiplicativo são similares. 
3.3 Caso 2: $\lambda=\frac{\sigma_{e}^{2}}{\sigma_{u}^{2}}$ conhecida

\subsubsection{Estimação dos parâmetros}

Nesta seção estudaremos a função escore corrigido derivada segundo a proposta de Bolfarine e Gimenez (1997).

Para fazer a estimação de parâmetros deve-se considerar que em modelos funcionais se trabalha com a suposição seguinte:

$$
\left(\begin{array}{c}
e_{i} \\
u_{i}
\end{array}\right) \sim N_{2}\left[\left(\begin{array}{l}
0 \\
1
\end{array}\right),\left(\begin{array}{cc}
\lambda \sigma_{u}^{2} & 0 \\
0 & \sigma_{u}^{2}
\end{array}\right)\right], \text { independentes } i=1, \ldots, n,
$$

enquanto em modelos estruturais a suposição a considerar pode ser definida por

$$
\left(\begin{array}{c}
X_{i} \\
e_{i} \\
u_{i}
\end{array}\right) \sim N_{3}\left[\left(\begin{array}{c}
\mu_{X} \\
0 \\
1
\end{array}\right),\left(\begin{array}{ccc}
\sigma_{X}^{2} & 0 & 0 \\
0 & \lambda \sigma_{u}^{2} & 0 \\
0 & 0 & \sigma_{u}^{2}
\end{array}\right)\right] \text {, independentes } i=1, \ldots, n
$$

A metodologia para estimar os parâmetros segue a linha de trabalho da seção 2.3.1 obtendo-se as equações (2.51) e (??), com uso das expressões (3.8) e (3.9). Neste caso, como no modelo aditivo, precisamos de um processo iterativo para estimar os parâmetros do modelo. O processo iterativo considera o algoritmo de Newton-Raphson que procede como segue.

Algoritmo para estimação dos parâmetros no método do escore corrigido

1. Escolha um valor inicial para $\sigma_{u}^{2}$.

2. Substitua o valor considerado na etapa anterior na expressão seguinte:

$$
\left(\begin{array}{cccc}
n & \sum_{i=1}^{n} t_{i,(1)} & \cdots & \sum_{i=1}^{n} t_{i,(p)} \\
\sum_{i=1}^{n} t_{i,(1)} & \sum_{i=1}^{n} t_{i,(2)} & \cdots & \sum_{i=1}^{n} t_{i,(p+1)} \\
\vdots & \vdots & \cdots & \vdots \\
\sum_{i=1}^{n} t_{i,(p)} & \sum_{i=1}^{n} t_{i,(p+1)} & \cdots & \sum_{i=1}^{n} t_{i,(2 p)}
\end{array}\right)\left(\begin{array}{c}
\hat{\beta}_{0}^{(m)} \\
\hat{\beta}_{1}^{(m)} \\
\vdots \\
\hat{\beta}_{p}^{(m)}
\end{array}\right)=\left(\begin{array}{c}
\sum_{i=1}^{n} Y_{i} \\
\sum_{i=1}^{n} Y_{i} t_{i,(1)} \\
\vdots \\
\sum_{i=1}^{n} Y_{i} t_{i,(p)}
\end{array}\right)
$$


lembrando que $t_{i,(s)}=\frac{W_{i}^{s}}{E\left(u_{i}^{s}\right)}$ e que $E\left(u_{i}^{s}\right)$ pode ser obtida segundo a expressão (3.9).

3. Agora com os valores $\beta^{(m)}$ obtidos como solução do sistema acima, devemos atualizar $\sigma_{u}^{2}$ de acordo com o método de Newton-Raphson:

$$
\sigma_{u}^{2(m+1)}=\sigma_{u}^{2(m)}+\frac{U^{*}\left(\sigma_{u}^{2(m)}\right)}{I^{*}\left(\sigma_{u}^{2(m)}\right)} .
$$

4. Retornar ao passo 2.

\subsubsection{Exemplos}

\section{Polinômio de primeiro grau}

Considerando as expressões (3.10) e (3.11), para $p=1$, temos

$$
\left(\begin{array}{cc}
n & \sum_{i=1}^{n} t_{i,(1)} \\
\sum_{i=1}^{n} t_{i,(1)} & \sum_{i=1}^{n} t_{i,(2)}
\end{array}\right)\left(\begin{array}{l}
\hat{\beta}_{0} \\
\hat{\beta}_{1}
\end{array}\right)=\left(\begin{array}{c}
\sum_{i=1}^{n} Y_{i} \\
\sum_{i=1}^{n} Y_{i} t_{i,(1)}
\end{array}\right),
$$

sendo a função escore corrigida para $\sigma_{u}^{2}$,

$$
U^{*}\left(\sigma_{u}^{2}\right)=-\frac{n}{2 \sigma_{u}^{2}}+\frac{1}{2 \lambda \sigma_{u}^{4}} \sum_{i=1}^{n}\left(Y_{i}^{2}+\beta_{0}^{2}+\beta_{1}^{2} t_{i,(2)}-2 Y_{i} \beta_{0}-2 Y_{i} \beta_{1} t_{i,(1)}-2 \beta_{0} \beta_{1} t_{i,(1)}\right) .
$$

Como $t_{i,(1)}=W_{i}$ e $t_{i,(2)}=\frac{W_{i}^{2}}{\sigma_{u}^{2}+1}$, temos

$$
U^{*}\left(\sigma_{u}^{2}\right)=-\frac{n}{2 \sigma_{u}^{2}}-\frac{n \beta_{1}^{2}\left(S_{W W}+\bar{W}^{2}\right)}{2 \lambda \sigma_{u}^{2}\left(\sigma_{u}^{2}+1\right)}+\frac{1}{2 \lambda \sigma_{u}^{2}} \sum_{i=1}^{n}\left(Y_{i}-\beta_{0}-\beta_{1} W_{i}\right)^{2},
$$

e a informação corrigida para $\sigma_{u}^{2}$ é dada por

$$
I^{*}\left(\sigma_{u}^{2}\right)=\frac{n}{2 \sigma_{u}^{4}}+\frac{n \beta_{1}^{2}\left(S_{W W}+\bar{W}^{2}\right)}{2 \lambda \sigma_{u}^{4}\left(\sigma_{u}^{2}+1\right)^{2}} .
$$

Considerando o método de Newton-Raphson modificado, temos o seguinte algoritmo,

1. Escolha um valor inicial para $\sigma_{u}^{2}$, faça $m=0$

2. faça 


$$
\hat{\beta}_{0}^{(m)}=\bar{Y}-\bar{W} \hat{\beta}_{1}^{(m)}
$$

com

$$
\hat{\beta}_{1}^{(m)}=\frac{\hat{\sigma}^{2(m)} S_{Y W}}{S_{W W}+\bar{W}\left(\sigma_{u}^{2(m)}+1\right)} .
$$

3. Atualizar $\sigma_{u}^{2}$ fazendo

$$
\hat{\sigma}_{u}^{2(m+1)}=\hat{\sigma}_{u}^{2(m)}+\frac{U^{*}\left(\sigma_{u}^{2(m)}\right)}{I^{*}\left(\sigma_{u}^{2(m)}\right)} .
$$

4. Se convergir, parar; caso contrario, faça $m=m+1$ e retornar ao passo (2).

Nas expressões acima,

$$
\begin{aligned}
& n^{2} S_{W W}=n \sum_{i=1}^{n} W_{i}^{2}-\left(\sum_{i=1}^{n} W_{i}\right)^{2} \\
& n^{2} S_{Y W}=n \sum_{i=1}^{n} Y_{i} W_{i}-\left(\sum_{i=1}^{n} Y_{i}\right)\left(\sum_{i=1}^{n} W_{i}\right) .
\end{aligned}
$$

\section{Polinômio de segundo grau}

Considerando as expressões (3.10) e (3.11), para $p=2$, temos

$$
\left(\begin{array}{ccc}
n & \sum_{i=1}^{n} t_{i,(1)} & \sum_{i=1}^{n} t_{i,(2)} \\
\sum_{i=1}^{n} t_{i,(1)} & \sum_{i=1}^{n} t_{i,(2)} & \sum_{i=1}^{n} t_{i,(3)} \\
\sum_{i=1}^{n} t_{i,(2)} & \sum_{i=1}^{n} t_{i,(3)} & \sum_{i=1}^{n} t_{i,(4)}
\end{array}\right)\left(\begin{array}{l}
\hat{\beta}_{0} \\
\hat{\beta}_{1} \\
\hat{\beta}_{2}
\end{array}\right)=\left(\begin{array}{c}
\sum_{i=1}^{n} Y_{i} \\
\sum_{i=1}^{n} Y_{i} t_{i,(1)} \\
\sum_{i=1}^{n} Y_{i} t_{i,(2)}
\end{array}\right)
$$

A função escore corrigido para $\sigma_{u}^{2}$ é, 


$$
\begin{aligned}
U^{*}\left(\sigma_{u}^{2}\right)= & \frac{n}{2 \sigma_{u}^{2}}-\frac{1}{2 \lambda \sigma_{u}^{4}} \sum_{i=1}^{n}\left[Y_{i}^{2}+\sum_{j=0}^{2} \hat{\beta}_{j}^{2} t_{i,(2 j)}-2 Y_{i} \sum_{j=0}^{2} \hat{\beta}_{j} t_{i,(j)}+\right. \\
& \left.+2 \sum_{\substack{j=0 \\
j<s}}^{2} \sum_{s=0}^{2} \hat{\beta}_{j} \hat{\beta}_{s} t_{i,(j+s)}\right] .
\end{aligned}
$$

Levando em conta que $t_{i,(1)}=W_{i}, t_{i,(2)}=\frac{W_{i}^{2}}{\sigma_{u}^{2}+1}, t_{i,(3)}=\frac{W_{i}^{3}}{3 \sigma_{u}^{2}+1}$ e $t_{i,(4)}=\frac{W_{i}^{4}}{3 \sigma_{u}^{4}+6 \sigma_{u}^{2}+1}$, a função escore corrigida para $\sigma_{u}^{2}$ pode ser escrita como

$$
\begin{aligned}
U^{*}\left(\sigma_{u}^{2}\right)= & -\frac{n}{2 \lambda \sigma_{u}^{2}}\left(\lambda+\frac{\beta_{1}^{2}}{n\left(\sigma_{u}^{2}+1\right)} \sum_{i=1}^{n} W_{i}^{2}+\frac{3 \beta_{2}^{2}\left(\sigma_{u}^{2}+2\right)}{n \sigma_{u}^{2}\left(3 \sigma_{u}^{4}+6 \sigma_{u}^{2}+1\right)} \sum_{i=1}^{n} W_{i}^{4}-\right. \\
& \left.-\frac{2 \beta_{2}}{n\left(\sigma_{u}^{2}+1\right)} \sum_{i=1}^{n} W_{i}^{2} Y_{i}+\frac{2 \beta_{0} \beta_{1}}{\left(\sigma_{u}^{2}+1\right)}+\frac{6 \beta_{1} \beta_{2}}{n\left(3 \sigma_{u}^{2}+1\right)} \sum_{i=1}^{n} W_{i}^{3}\right)+ \\
& +\frac{1}{2 \lambda \sigma_{u}^{4}} \sum_{i=1}^{n}\left(Y_{i}-\beta_{0}-\beta_{1} W_{i}-\beta_{2} W_{i}^{2}\right)^{2}
\end{aligned}
$$

sendo a informação observada para $\sigma_{u}^{2}$ dada por

$$
\begin{aligned}
I^{*}\left(\sigma_{u}^{2}\right)= & \frac{n}{\lambda \sigma_{u}^{2}}\left[\frac{\lambda}{\sigma_{u}^{2}}+\frac{\beta_{1}^{2}}{n \sigma_{u}^{2}\left(\sigma_{u}^{2}+1\right)} \sum_{i=1}^{n} W_{i}^{2}+\frac{3 \beta_{2}^{2}\left(15 \sigma_{u}^{6}+36 \sigma_{u}^{4}+15 \sigma_{u}^{2}+2\right)}{n \sigma_{u}^{4}\left(3 \sigma_{u}^{4}+6 \sigma_{u}^{2}+1\right)^{2}} \sum_{i=1}^{n} W_{i}^{4}-\right. \\
& \left.-\frac{2 \beta_{2} \sigma_{u}^{2}}{n\left(\sigma_{u}^{2}+1\right)^{2}} \sum_{i=1}^{n} Y_{i} W_{i}+\frac{2 \beta_{0} \beta_{2} \sigma_{u}^{2}}{\left(\sigma_{u}^{2}+1\right)^{2}}+\frac{6 \beta_{1} \beta_{2}\left(3 \sigma_{u}^{2}+2\right)}{n\left(3 \sigma_{u}^{2}+1\right)^{2}} \sum_{i=1}^{n} W_{i}^{3}\right),
\end{aligned}
$$

O algoritmo de estimação dos parâmetros funciona do modo seguinte:

1. Escolha um valor inicial para $\sigma_{u}^{2}$; faça $m=0$.

2. Faça

$$
\hat{\beta}_{0}^{(m)}=\bar{Y}-\bar{W} \hat{\beta}_{1}^{(m)}-\frac{\left(S_{W W}+\bar{W}^{2}\right) \hat{\beta}_{2}^{(m)}}{\left(\hat{\sigma}_{u}^{2(m)}+1\right)},
$$




$$
\hat{\beta}_{1}^{(m)}=\left[\frac{\left(\hat{\sigma}_{u}^{2(m)}+1\right) S_{Y W}}{S_{W W}-\bar{W}^{2} \hat{\sigma}_{u}^{2(m)}}\right]-\mathbf{B}^{(m)} \hat{\beta}_{2}^{(m)},
$$

com

$$
\hat{\beta}_{2}^{(m)}=\frac{S_{Y W^{2}}}{\mathbf{A}^{(m)}+\mathbf{B}^{(m)}}-\left[\frac{\left(\hat{\sigma}_{u}^{2(m)}+1\right) S_{Y W}}{S_{W W}-\bar{W}^{2} \sigma_{u}^{2(m)}}\right]\left[\frac{\mathbf{B}^{(m)}}{\mathbf{A}^{(m)}+\mathbf{B}^{(m)}}\right],
$$

onde

$$
\begin{aligned}
\mathbf{A}^{(m)} & =\frac{\left(\hat{\sigma}_{u}^{2(m)}+1\right) S_{W W^{2}}-2 \hat{\sigma}_{u}^{2(m)}\left(S_{W W}+\bar{W}^{2}\right)^{2}}{3 \hat{\sigma}^{4(m)}+6 \hat{\sigma}_{u}^{2(m)}+1}, \\
\mathbf{B}^{(m)} & =\frac{\left(\sigma_{u}^{2(m)}+1\right) S_{W W^{2}}-2 \bar{W} \sigma_{u}^{2(m)}\left(S_{W W}+\bar{W}^{2}\right)}{3 \sigma_{u}^{2(m)}+1},
\end{aligned}
$$

e

$$
\begin{aligned}
n^{2} S_{W W} & =n \sum_{i=1}^{n} W_{i}^{2}-\left(\sum_{i=1}^{n} W_{i}\right)^{2}, \\
n^{2} S_{W W^{2}} & =n \sum_{i=1}^{n} W_{i}^{3}-\left(\sum_{i=1}^{n} W_{i}\right)\left(\sum_{i=1}^{n} W_{i}^{2}\right), \\
n^{2} S_{W^{2} W^{2}} & =n \sum_{i=1}^{n} W_{i}^{4}-\left(\sum_{i=1}^{n} W_{i}^{2}\right)^{2}, \\
n^{2} S_{Y W} & =n \sum_{i=1}^{n} Y_{i} W_{i}-\left(\sum_{i=1}^{n} Y_{i}\right)\left(\sum_{i=1}^{n} W_{i}\right), \\
n^{2} S_{Y W^{2}} & =n \sum_{i=1}^{n} Y_{i} W_{i}^{2}-\left(\sum_{i=1}^{n} Y_{i}\right)\left(\sum_{i=1}^{n} W_{i}^{2}\right) .
\end{aligned}
$$

3. Atualizar $\sigma_{u}^{2}$, fazendo

$$
\hat{\sigma}_{u}^{2(m+1)}=\hat{\sigma}_{u}^{2(m)}+\frac{U^{*}\left(\sigma_{u}^{2(m)}\right)}{I^{*}\left(\sigma^{2(m)}\right)}
$$

4. Se convergiu parar; caso contrario, faça $m=m+1$ e retorne ao passo (2). 


\subsubsection{Aplicações numéricas}

Para as aplicações numéricas consideramos duas situações, um estudo da simulação e uma situação prática.

Um estudo de simulação.

Para esta simulação consideramos um modelo funcional em um polinômio de grau dois. Efetuamos 1.000 replicações para estudar a convergência dos estimadores e os seguintes parâmetros foram tomados em consideração:

1. Para $n$ especificado, considerar $\quad X_{i}=\operatorname{Binomial}(n ; 0,5), \quad u_{i} \sim N(1 ; 0,05) \quad$ e $e_{i} \sim N(0 ; 1)$ para $i=1,2, \ldots, n$.

2. Obtenção dos dados:

$$
\begin{aligned}
W_{i} & =X_{i} u_{i}, \\
Y_{i} & =\beta_{0}+\beta_{1} X_{i}+\beta_{2} X_{i}^{2}+e_{i},
\end{aligned}
$$

sendo $\beta_{0}=\beta_{1}=\beta_{2}=1$.

3. Para os efeitos práticos, deve-se considerar o processo iterativo: "Algoritmo para estimação dos parâmetros no caso de Escore Corrigida".

\section{Resultados}

Na tabela a seguir é o resultado da implementação da metodologia apresentada na linguagem Ox (Doornik, 1998), e resume os resultados obtidos pelo método "naive" e "escore corrigido" apresentando as estimativas, os desvios padrões e o erro quadrático médio (EQM) para cada metodologia. 
Tabela 3.3. Estimativas segundo os métodos "naive"e "escore corrigido" para diferentes tamanhos de amostra.

\begin{tabular}{|c|c|c|c|c|c|c|c|}
\hline \multirow[t]{2}{*}{$\mathrm{n}$} & \multirow[t]{2}{*}{ Parâmetro } & \multicolumn{3}{|c|}{ Naive } & \multicolumn{3}{|c|}{ Escore corrigido } \\
\hline & & Estimativa & Desv.Pad. & EQM & Estimativa & Desv.Pad. & EQM \\
\hline \multirow{4}{*}{5000} & $\hat{\beta}_{0}$ & 1,45541 & 0,06726 & 0,70190 & 0,98836 & 0,09568 & 0,81499 \\
\hline & $\hat{\beta}_{1}$ & 0,73549 & 0,08937 & 0,45840 & 0,96160 & 0,35259 & 0,04139 \\
\hline & $\hat{\beta}_{2}$ & 0,65522 & 0,03074 & 2,75173 & 1,00743 & 0,12508 & 0,16945 \\
\hline & $\hat{\sigma}_{e}^{2}$ & 2,82161 & - & 3,03813 & 0,22933 & - & 0,49092 \\
\hline \multirow{4}{*}{6000} & $\hat{\beta}_{0}$ & 1,32373 & 0,03963 & 0,89150 & 1,05442 & 0,13553 & 0,04155 \\
\hline & $\hat{\beta}_{1}$ & 0,73662 & 0,06116 & 0,45144 & 1,02286 & 0,35192 & 0,03582 \\
\hline & $\hat{\beta}_{2}$ & 0,65750 & 0,05342 & 2,95609 & 0,99281 & 0,09676 & 0,13004 \\
\hline & $\hat{\sigma}_{e}^{2}$ & 2,51670 & - & 2,28504 & 0,26779 & - & 0,09304 \\
\hline \multirow{4}{*}{7000} & $\hat{\beta}_{0}$ & 1,27637 & 0,07194 & 0,61855 & 1,05532 & 0,09608 & 0,21479 \\
\hline & $\hat{\beta}_{1}$ & 0,76080 & 0,07221 & 0,65348 & 0,95655 & 0,27234 & 0,03489 \\
\hline & $\hat{\beta}_{2}$ & 0,62510 & 0,03658 & 1,24128 & 0,99382 & 0,05992 & 0,18398 \\
\hline & $\hat{\sigma}_{e}^{2}$ & 2,71499 & - & 1,92783 & 0,21109 & - & 0,49164 \\
\hline \multirow{4}{*}{8000} & $\hat{\beta}_{0}$ & 1,32021 & 0,07038 & 0,84191 & 1,04245 & 0,09726 & 0,44846 \\
\hline & $\hat{\beta}_{1}$ & 0,59478 & 0,08483 & 0,60268 & 1,00311 & 0,19326 & 0,03253 \\
\hline & $\hat{\beta}_{2}$ & 0,70573 & 0,02411 & 1,14961 & 1,00797 & 0,14584 & 0,17164 \\
\hline & $\hat{\sigma}_{e}^{2}$ & 2,83781 & - & 2,93282 & 0,26210 & - & 0,41846 \\
\hline \multirow{4}{*}{9000} & $\hat{\beta}_{0}$ & 1,36812 & 0,05217 & 0,64416 & 1,04353 & 0,09444 & 0,28291 \\
\hline & $\hat{\beta}_{1}$ & 0,72324 & 0,06921 & 0,66959 & 1,03396 & 0,23717 & 0,03275 \\
\hline & $\hat{\beta}_{2}$ & 0,68556 & 0,06883 & 2,16227 & 1,01087 & 0,10774 & 0,18260 \\
\hline & $\hat{\sigma}_{e}^{2}$ & 2,90807 & - & 3,37423 & 0,21006 & - & 0,39237 \\
\hline
\end{tabular}

Como se pode observar na tabela acima, os estimadores dos parâmetros $\beta$ obtidos pelo método do escore corrigido atingem aos parâmetros propostos em nossa simulação, da mesma maneira os valores de $\sigma_{u}^{2}$ esta próximos dos valores fixados na simulação, enquanto as estimativas obtidas pelo método "naive" não se aproximam dos verdadeiros valores. Uma representação gráfica é apresentada a seguir 


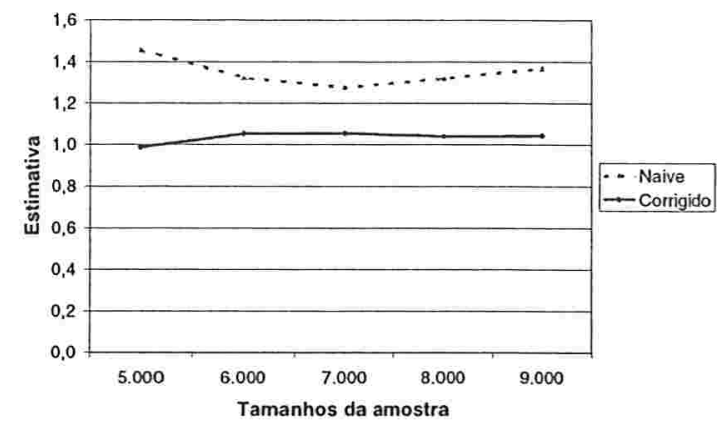

(a) Estimativa para $\beta_{0}$

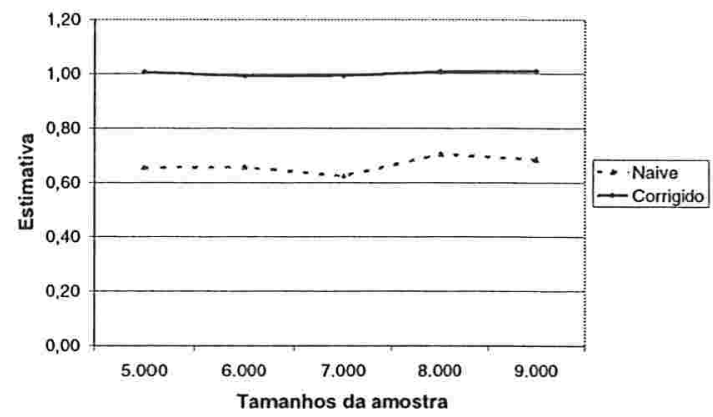

(c) Estimativa para $\beta_{2}$

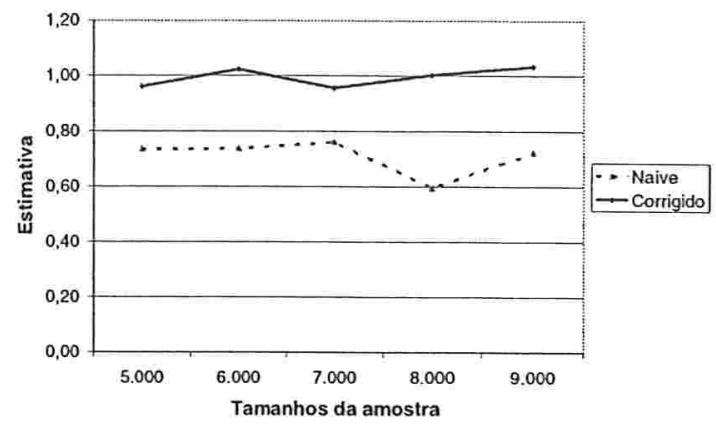

(b) Estimativa para $\beta_{1}$

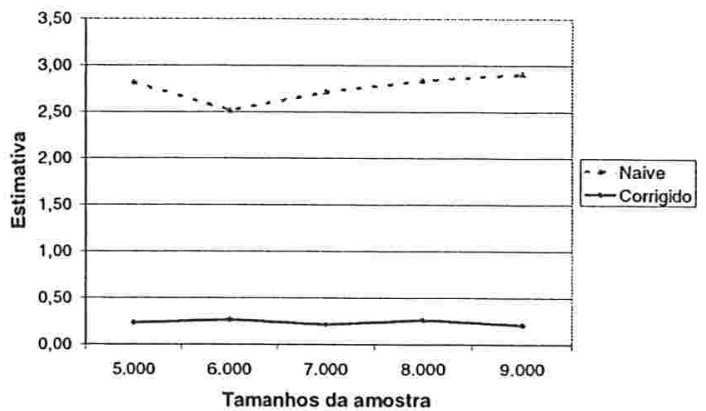

(d) Estimativa para $\sigma_{e}^{2}$

Fig. 3.2. Comportamento das estimativas "naive"e "escore corrigido"segundo o tamanho da amostra

Na figura de acima podemos observar que os valores correspondentes aos estimativas para os parâmetros "naive" não conseguem atingir os valores implementados na presente simulação, enquanto as estimativas para os parâmetros "escore corrigido" se aproximam adequadamente. 


\section{b. Um caso prático.}

Para avaliar a metodologia proposta, consideramos a informação apresentada por Fuller (1987) (pag. 248), estes dados encontram-se no anexo deste trabalho e as variáveis consideradas são $Y_{2}$ e $W$.

\section{Resultados}

Após a implementação da metodologia apresentada no Ox (Doornik, 1998) um resumo dos resultados são apresentados na seguinte tabela, considerando-se as estimativas do modelo de escore corrigido no caso aditivo frente ao modelo de escore corrigido no caso multiplicativo.

Tabela 3.4. Estimativas segundo o método de escore corrigido

\begin{tabular}{|c|c|c||c|c|}
\hline \multirow{2}{*}{ Parâmetro } & \multicolumn{2}{|c||}{ Aditivo } & \multicolumn{2}{c|}{ Multiplicativo } \\
\cline { 2 - 5 } & Estimativa & Desv.Pad. & Estimativa & Desv.Pad. \\
\hline$\beta_{0}$ & $-0,023631$ & 0,10310 & $-0,052640$ & 0,082671 \\
$\beta_{1}$ & 0,004183 & 0,09509 & 0,003048 & 0,072302 \\
$\beta_{2}$ & 1,027800 & 0,22073 & 1,008500 & 0,146250 \\
$\sigma_{u}^{2}$ & 0,087207 & - & 0,090000 & - \\
\hline
\end{tabular}

Observemos na tabela acima que as estimativas obtidas pelo método de escore corrigido tanto para o modelo aditivo quanto para o modelo multiplicativo tem estimativas muito similares.

\subsection{Caso 3: Variâncias heterogêneas}

\subsubsection{Estimação dos parâmetros}

Como as expressões de $\hat{\beta}$ são similares nos casos multiplicativo e aditivo, consideramos a metodologia implementada na seção 2.5 , considerando a equação (3.8) para a obtenção dos $t_{i,(s)}$. Para a estimação dos parâmetros devemos considerar o sistema de equações lineares abaixo, de acordo com (2.86) 


$$
\left(\begin{array}{cccc}
\sum_{i=1}^{n} \frac{1}{\sigma_{e_{i}}^{2}} & \sum_{i=1}^{n} \frac{t_{i,(1)}}{\sigma_{e_{i}}^{2}} & \ldots & \sum_{i=1}^{n} \frac{t_{i,(p)}}{\sigma_{e_{i}}^{2}} \\
\sum_{i=1}^{n} \frac{t_{i,(1)}}{\sigma_{e_{i}}^{2}} & \sum_{i=1}^{n} \frac{t_{i,(2)}}{\sigma_{e_{i}}^{2}} & \ldots & \sum_{i=1}^{n} \frac{t_{i,(p+1)}}{\sigma_{e_{i}}^{2}} \\
\vdots & \vdots & \ldots & \vdots \\
\sum_{i=1}^{n} \frac{t_{i,(p)}}{\sigma_{e_{i}}^{2}} & \sum_{i=1}^{n} \frac{t_{i,(p+1)}}{\sigma_{e_{i}}^{2}} & \ldots & \sum_{i=1}^{n} \frac{t_{i,(2 p)}}{\sigma_{e_{i}}^{2}}
\end{array}\right)\left(\begin{array}{c}
\hat{\beta}_{0} \\
\hat{\beta}_{1} \\
\vdots \\
\hat{\beta}_{p}
\end{array}\right)=\left(\begin{array}{c}
\sum_{i=1}^{n} \frac{Y_{i}}{\sigma_{e_{i}}^{2}} \\
\sum_{i=1}^{n} \frac{Y_{i} t_{i,(1)}}{\sigma_{e_{i}}^{2}} \\
\vdots \\
\sum_{i=1}^{n} \frac{Y_{i} t_{i,(p)}}{\sigma_{e_{i}}^{2}}
\end{array}\right)
$$

A solução deste sistema de equações permite-nos obter os estimadores do modelo.

\subsubsection{Exemplos}

\section{Polinômio de primeiro grau}

Considerando a expressão apresentada acima para $\mathrm{p}=1$, temos

$$
\left(\begin{array}{cc}
\sum_{i=1}^{n} m_{i} & \sum_{i=1}^{n} m_{i} t_{i,(1)} \\
\sum_{i=1}^{n} m_{i} t_{i,(1)} & \sum_{i=1}^{n} m_{i} t_{i,(2)}
\end{array}\right)\left(\begin{array}{l}
\hat{\beta}_{0} \\
\hat{\beta}_{1}
\end{array}\right)=\left(\begin{array}{c}
\sum_{i=1}^{n} m_{i} Y_{i} \\
\sum_{i=1}^{n} m_{i} Y_{i} t_{i,(1)}
\end{array}\right),
$$

onde $t_{i,(1)}=W_{i}, t_{i,(2)}=\frac{W_{i}^{2}}{\sigma_{u}^{2}+1}$ e $m_{i}=\frac{1}{\sigma_{e_{i}}^{2}}$, de maneira que

$$
\begin{gathered}
\hat{\beta}_{0}=\frac{\sum_{i=1}^{n} m_{i} Y_{i}}{\sum_{i=1}^{n} m_{i}}-\frac{\sum_{i=1}^{n} m_{i} W_{i}}{\sum_{i=1}^{n} m_{i}} \hat{\beta}_{1}, \\
\hat{\beta}_{1}=\frac{S_{Y W}}{S_{W W}-\sum_{i=1}^{n} \frac{m_{i} \sigma_{u_{i}}^{2} W_{i}^{2}}{\left(\sigma_{u_{i}}^{2}+1\right)}}
\end{gathered}
$$

onde 


$$
\begin{aligned}
\left(\sum_{i=1}^{n} m_{i}\right) S_{W W} & =\sum_{i=1}^{n} m_{i} W_{i}^{2}-\frac{\left(\sum_{i=1}^{n} m_{i} W_{i}\right)^{2}}{\sum_{i=1}^{n} m_{i}} \\
\left(\sum_{i=1}^{n} m_{i}\right) S_{Y W} & =\sum_{i=1}^{n} m_{i} Y_{i} W_{i}-\frac{\left(\sum_{i=1}^{n} m_{i} Y_{i}\right)\left(\sum_{i=1}^{n} m_{i} W_{i}\right)}{\sum_{i=1}^{n} m_{i}} .
\end{aligned}
$$

Gostaríamos de esclarecer que os estimadores acima não foram ainda obtidos na literatura.

\section{Polinômio de segundo grau}

Considerando a expressão apresentada acima para $\mathrm{p}=2$, temos

$$
\left(\begin{array}{ccc}
\sum_{i=1}^{n} m_{i} & \sum_{i=1}^{n} m_{i} t_{i,(1)} & \sum_{i=1}^{n} m_{i} t_{i,(2)} \\
\sum_{i=1}^{n} m_{i} t_{i,(1)} & \sum_{i=1}^{n} m_{i} t_{i,(2)} & \sum_{i=1}^{n} m_{i} t_{i,(3)} \\
\sum_{i=1}^{n} m_{i} t_{i,(2)} & \sum_{i=1}^{n} m_{i} t_{i,(3)} & \sum_{i=1}^{n} m_{i} t_{i,(4)}
\end{array}\right)\left(\begin{array}{l}
\hat{\beta}_{0} \\
\hat{\beta}_{1} \\
\hat{\beta}_{2}
\end{array}\right)=\left(\begin{array}{c}
\sum_{i=1}^{n} m_{i} Y_{i} \\
\sum_{i=1}^{n} m_{i} Y_{i} t_{i,(1)} \\
\sum_{i=1}^{n} m_{i} Y_{i} t_{i,(2)}
\end{array}\right),
$$

segue de (3.8) que $t_{i,(1)}=W_{i}, t_{i,(2)}=\frac{W_{i}^{2}}{\sigma_{u_{i}}^{2}+1}, t_{i,(3)}=\frac{W_{i}^{3}}{3 \sigma_{u_{i}}^{2}+1}, t_{i,(4)}=\frac{W_{i}^{4}}{3 \sigma_{u_{i}}^{4}+6 \sigma_{u_{i}}^{2}+1} \mathrm{e}$ lembrando que $m_{i}=\frac{1}{\sigma_{e_{i}}^{2}}$, temos

$$
\begin{aligned}
& \hat{\beta}_{0}=\frac{\sum_{i=1}^{n} m_{i} Y_{i}}{\sum_{i=1}^{n} m_{i}}-\frac{\sum_{i=1}^{n} m_{i} W_{i}}{\sum_{i=1}^{n} m_{i}} \hat{\beta}_{1}-\frac{\sum_{i=1}^{n} m_{i} W_{i}^{2}}{\sigma_{u_{i}}^{2}+1} \frac{\hat{\beta}_{2}}{\sum_{i=1}^{n} m_{i}}, \\
& \hat{\beta}_{1}=\frac{1}{\mathrm{~A}} \sum_{i=1}^{n} m_{i} S_{Y W}-\mathbf{B} \hat{\beta}_{2}, \\
& \hat{\beta}_{2}=\frac{\mathrm{C}}{\mathrm{D}}+\frac{\mathrm{B}}{\mathrm{AD}} \sum_{i=1}^{n} m_{i} S_{Y W},
\end{aligned}
$$


onde

$$
\begin{aligned}
& \mathrm{A}=\sum_{i=1}^{n} m_{i} S_{W W}-\sum_{i=1}^{n} \frac{m_{i} \sigma_{u_{i}}^{2} W_{i}^{2}}{\sigma_{u_{i}}^{2}+1}, \\
& \mathrm{~B}=\sum_{i=1}^{n} \frac{m_{i} W_{i}^{3}}{3 \sigma_{u_{i}}^{2}+1}-\frac{\sum_{i=1}^{n} m_{i} W_{i}}{\sum_{i=1}^{n} m_{i}} \sum_{i=1}^{n} \frac{m_{i} W_{i}^{2}}{\sigma_{u_{i}}^{2}+1}, \\
& \mathrm{C}=\sum_{i=1}^{n} \frac{m_{i} Y_{i} W_{i}^{2}}{\sigma_{u_{i}}^{2}+1}-\sum_{i=1}^{n} \frac{m_{i} W_{i}^{2}}{\sigma_{u_{i}}^{2}+1} \frac{\sum_{i=1}^{n} m_{i} Y_{i}}{\sum_{i=1}^{n} m_{i}}, \\
& \mathrm{D}=\mathrm{B} \sum_{i=1}^{n} \frac{m_{i} W_{i}^{3}}{3 \sigma_{u_{i}}^{2}+1}\left(\sum_{i=1}^{n} m_{i} S_{Y W}-1\right)+\sum_{i=1}^{n} \frac{m_{i} W_{i}^{4}}{3 \sigma_{u_{i}}^{4}+6 \sigma_{u_{i}}^{2}+1}-\frac{1}{\sum_{i=1}^{n} m_{i}}\left(\sum_{i=1}^{n} \frac{m_{i} W_{i}^{2}}{\sigma_{u_{i}}^{2}+1}\right)^{2},
\end{aligned}
$$

$$
\begin{aligned}
& \sum_{i=1}^{n} m_{i} S_{W W}=\sum_{i=1}^{n} m_{i} W_{i}^{2}-\frac{\left(\sum_{i=1}^{n} m_{i} W_{i}\right)^{2}}{\sum_{i=1}^{n} m_{i}}, \\
& \sum_{i=1}^{n} m_{i} S_{Y W}=\sum_{i=1}^{n} m_{i} Y_{i} W_{i}-\frac{\sum_{i=1}^{n} m_{i} W_{i} \sum_{i=1}^{n} m_{i} Y_{i}}{\sum_{i=1}^{n} m_{i}} .
\end{aligned}
$$

\subsubsection{Aplicações numéricas}

Para as aplicações numéricas realizamos uma simulação de Monte Carlo para avaliar os parâmetros e também uma aplicação em um modelo usado na detecção do viés analítica publicado por Galea-Rojas, de Castilho, Bolfarine e de Castro (2003). 
a) Um estudo de simulação.

Considerando um valor de $n$ fixo, os elementos do modelo quadrático podem ser definidos a partir dos passos abaixo:

- Gerar variáveis da forma $X_{i} \sim U(40 ; 80)$.

- Gerar $n$ variáveis independentes para $u_{i}$, onde $u_{i} \sim N\left(1 ; \sigma_{u_{i}}^{2}\right)$.

- Gerar n variáveis independentes para $e_{i}$, onde $e_{i} \sim N\left(0 ; \sigma_{e_{i}}^{2}\right)$.

- Os valores de $\sigma_{u_{i}}^{2}$ serão obtidos a partir de uma distribuição uniforme em $(0 ; 0,5)$ enquanto $\sigma_{e_{i}}^{2}$ serão obtidos a partir de uma distribuição uniforme em $(0 ; 0,05)$.

- Gerar n variáveis independentes para $W_{i}=X_{i i}$.

- Obter para $Y_{i}=\beta_{0}+\beta_{1} X_{i}+\beta_{1} X_{i}^{2}+e_{i}$, sendo os valores de $\beta_{0}=1, \beta_{1}=1$ e $\beta_{2}=1$.

\section{Resultados}

Após implementada a metodologia na linguagem Ox (Doornik, 1998), os resultados obtidos são apresentados na tabela a seguir 
Tabela 3.5. Estimativas segundo os métodos "naive"e "escore corrigido" para diferentes tamanhos de amostra.

\begin{tabular}{|c|c|c|c|c||c|c|c|}
\hline \multirow{2}{*}{$\mathrm{n}$} & Parâmetro & \multicolumn{3}{|c||}{ Naive } & \multicolumn{3}{c|}{ Escore corrigido } \\
\cline { 2 - 8 } & & Estimativa & Desv.Pad. & EQM & Estimativa & Desv.Pad. & EQM \\
\hline \multirow{4}{*}{5000} & $\hat{\beta}_{0}$ & 1,21249 & 0,59676 & 0,60782 & 0,98639 & 0,57699 & 0,71449 \\
& $\hat{\beta}_{1}$ & 1,41770 & 0,20090 & 0,16607 & 0,99433 & 0,34213 & 0,04850 \\
& $\hat{\beta}_{2}$ & 0,93610 & 0,02364 & 0,01033 & 0,99204 & 0,02924 & 0,00073 \\
\hline \multirow{3}{*}{6000} & $\hat{\beta}_{0}$ & 1,21867 & 0,49487 & 0,39585 & 1,00702 & 0,58175 & 0,57051 \\
& $\hat{\beta}_{1}$ & 1,23705 & 0,24910 & 0,38741 & 0,98364 & 0,31725 & 0,05921 \\
& $\hat{\beta}_{2}$ & 0,93898 & 0,04161 & 0,00483 & 0,99546 & 0,04176 & 0,00187 \\
\hline \multirow{3}{*}{7000} & $\hat{\beta}_{0}$ & 1,19404 & 0,63631 & 0,76630 & 1,02672 & 0,75499 & 0,62193 \\
& $\hat{\beta}_{1}$ & 1,25169 & 0,27943 & 0,18340 & 0,99310 & 0,25700 & 0,17179 \\
& $\hat{\beta}_{2}$ & 0,91209 & 0,03518 & 0,00798 & 0,99625 & 0,02138 & 0,00083 \\
\hline \multirow{3}{*}{8000} & $\hat{\beta}_{0}$ & 1,22684 & 0,48879 & 0,84694 & 1,02377 & 0,49102 & 0,54898 \\
& $\hat{\beta}_{1}$ & 1,23036 & 0,17417 & 0,31152 & 0,99361 & 0,30723 & 0,10072 \\
& $\hat{\beta}_{2}$ & 0,92119 & 0,01801 & 0,01078 & 0,99424 & 0,02001 & 0,00183 \\
\hline \multirow{3}{*}{9000} & $\hat{\beta}_{0}$ & 1,23219 & 0,36816 & 0,88695 & 1,01648 & 0,82497 & 0,52792 \\
& $\hat{\beta}_{1}$ & 1,30309 & 0,20946 & 0,40615 & 1,00345 & 0,37062 & 0,17920 \\
& $\hat{\beta}_{2}$ & 0,91069 & 0,04160 & 0,00525 & 0,99168 & 0,02147 & 0,00162 \\
\hline
\end{tabular}

Da tabela podemos observar que a metodologia proposta leva a estimativas muito próximas aos valores fixados na presente simulação, pode-se observar também que as estimativas obtidas pelo método naive não se aproximam aos valores fixados. Uma representação gráfica é apresentada a seguir 


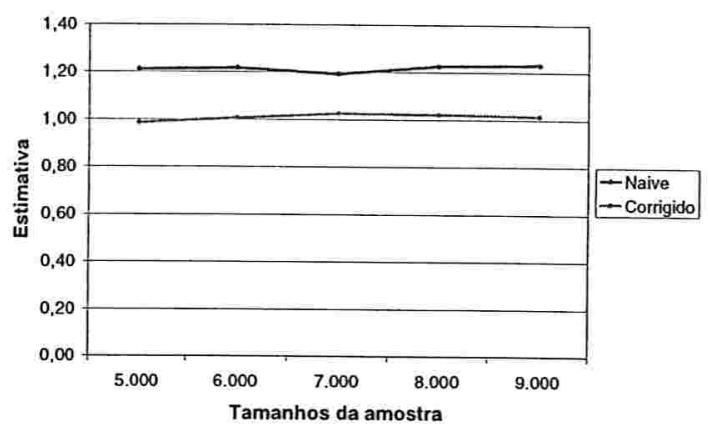

(a) Estimativa para $\beta_{0}$

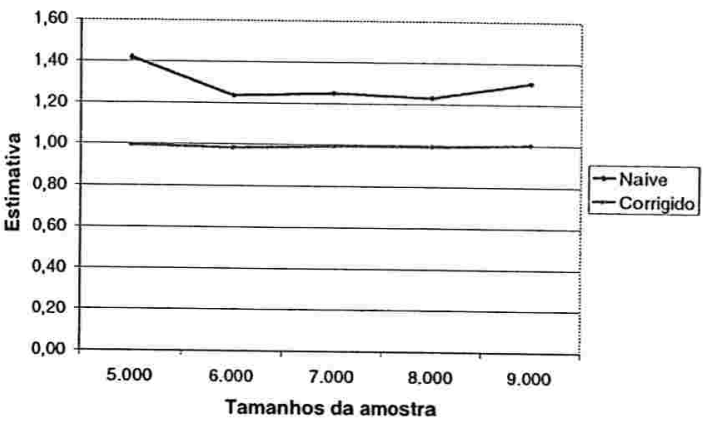

(b) Estimativa para $\beta_{1}$

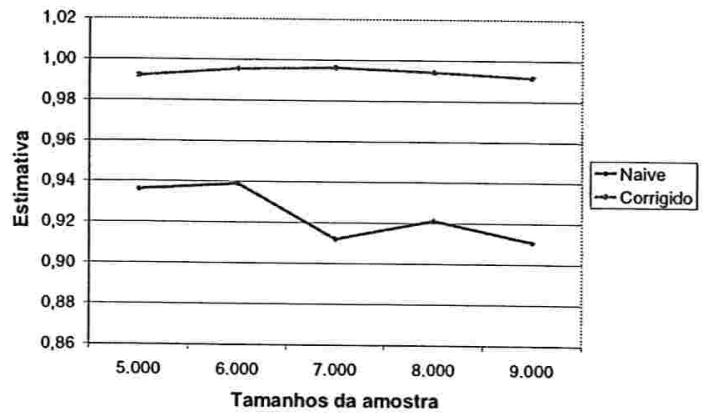

(c) Estimativa para $\beta_{2}$

Fig. 3.3. Comportamento das estimativas "naive"e "escore corrigido"segundo o tamanho da amostra

\section{a) Um caso prático.}

Para a aplicação prática considerou-se o exemplo 4 em Galea-Rojas, de Castilho, Bolfarine e de Castro (2003) com a finalidade de comparar a presente metodologia com a metodologia apresentada no capítulo 2 , onde foi utilizado este mesmo exemplo, os resultados se apresentam na tabela a seguir 
Tabela 3.6. Estimativas segundo o método de escore corrigido

\begin{tabular}{|c|c|c||c|c|}
\hline \multirow{2}{*}{ Parâmetro } & \multicolumn{2}{|c||}{ Aditivo } & \multicolumn{2}{c|}{ Multiplicativo } \\
\cline { 2 - 5 } & Estimativa & Desv.Pad. & Estimativa & Desv.Pad. \\
\hline$\beta_{0}$ & 0,47412 & 0,07409 & 0,017457 & 0,011139 \\
$\beta_{1}$ & $-1,67880$ & 0,36417 & 0,930970 & 0,091829 \\
$\beta_{2}$ & 0,63764 & 0,13876 & $-0,020733$ & 0,048761 \\
\hline
\end{tabular}

Observe-se que as estimativas de ambos modelos são um pouco diferentes, para observar melhor apresentamos um gráfico das tendências de ambos modelos

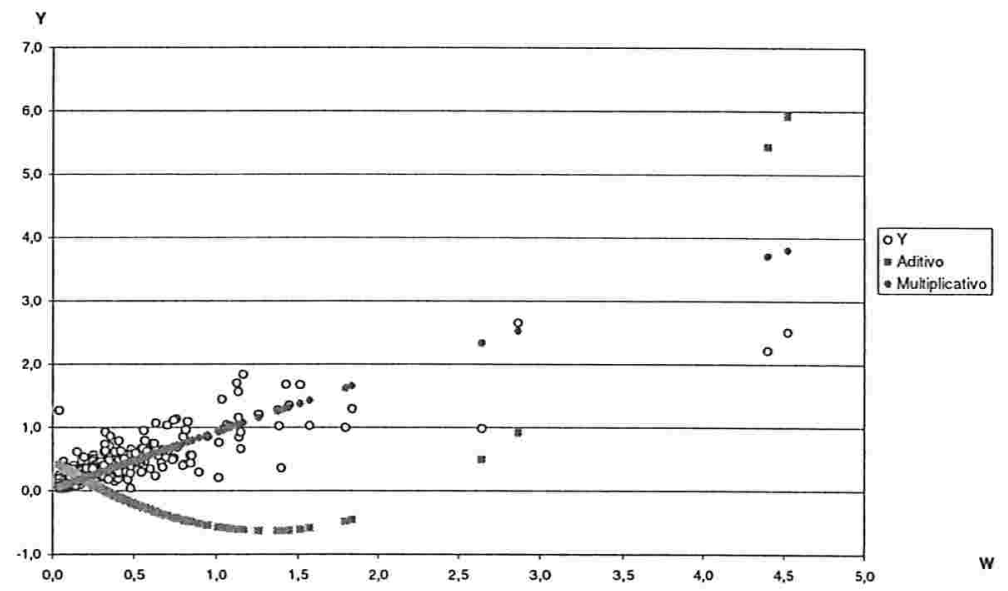

Fig. 3.4. Gráfico comparativo dos modelos aditivo e multiplicativo na metodologia escore corrigido

Do gráfico acima, pode-se observar que os dados se ajustaram melhor a um modelo multiplicativo que a um modelo aditivo. 


\section{CAPÍTULO}

\section{Calibração Comparativa}

\subsection{Introdução}

A calibração estatística é importante em aplicações práticas em diversas áreas. A ccalibração estatística é o processo através do qual a escala de um instrumento de medição é determinada ou ajustada a partir de um instrumento de referência.

Deve-se diferenciar a definição entre calibração absoluta e calibração comparativa. A calibração absoluta pode entender-se como uma forma de predição inversa que alguns autores chamam de regressão inversa. Na calibração comparativa pretende-se determinar a relação entre diversos testes ou instrumentos que fornecem medidas indiretas das mesmas unidades experimentais.

Suponha que dispomos de $r+1(r \geq 1)$ instrumentos para medir uma característica ou resposta $X_{i}$ em um grupo de n unidades. Um modelo freqüentemente adotado para este tipo de problema na literatura é dado por Barnett, (1969):

$$
\begin{aligned}
& \mathrm{Y}_{i}=\beta_{0}+\beta_{1} X_{i}+\mathrm{e}_{i}, \\
& W_{i}=X_{i}+u_{i},
\end{aligned}
$$

onde $Y_{i}=\left(Y_{i, 1}, Y_{i, 2}, \ldots, Y_{i, r}\right)^{t}, e_{i}=\left(e_{i, 1}, e_{i, 2}, \ldots, e_{i, r}\right)^{t}$, para $i=1,2, \ldots, n$, são vetores aleatórios de dimensão $r \times 1, \beta_{0}=\left(\beta_{0,1}, \beta_{0,2}, \ldots, \beta_{0, r}\right)^{t}$ e $\beta_{1}=\left(\beta_{1,1}, \beta_{1,2}, \ldots, \beta_{1, r}\right)^{t}$ são parâmetros sobre os quais desejamos fazer inferências, sendo os $\beta_{0}$ 's os vícios aditivos e os 
$\beta_{1}$ 's os vícios multiplicativos, $X_{i}$ representa o verdadeiro valor da característica de interesse na $i$-ésima unidade, $Y_{i j}$ corresponde à medida fornecida pelo $k$-ésimo instrumento para a característica da $i$-ésima unidade, $k=1,2, \ldots, r$ e $i=1,2, \ldots, n$.

A equação (4.2) considera que um dos instrumentos mede a quantidade desconhecida $X_{i}$ correspondente à $i$-ésima unidade sem vício. Segundo Barnnet (1969), este instrumento é chamado de instrumento de referência ou padrão. Para esta análise é assumido que $\mathbf{e}_{i} \sim$ $N_{r}(0, \Sigma)$ independentes, com $\Sigma=\left(\sigma_{1}^{2}, \ldots, \sigma_{r}^{2}\right)$ e $u_{i} \sim N\left(0, \sigma_{u}^{2}\right)$ independentes, para $i=$ $1,2, \ldots, n$, sendo $e_{i}$ e $u_{i}$ independentes.

No presente capítulo pretende-se estender o modelo de calibração comparativa usual para o polinomial, que não foi tratado na literatura. O modelo de calibração comparativa polinomial é expresso por

$$
\begin{aligned}
\mathrm{Y}_{i} & =\sum_{j=1}^{p} \beta_{j} X_{i}^{j}+\mathrm{e}_{i}, \\
W_{i} & =X_{i}+u_{i} \quad i=1, \ldots, n,
\end{aligned}
$$

onde os $\beta_{0}$ é o vício aditivo e os $\beta_{1}, \beta_{2}, \ldots, \beta_{p}$ são os vícios multiplicativos. No modelo de calibração polinomial, a expressão (4.4) é conhecida na literatura como a equação do instrumento de referência (ou padrão), que fornece as medições não viciadas.

O presente capítulo está estruturado do modo seguinte. Na seção 4.2 será estudado o caso em que as variâncias dos erros são homogêneas, isto é, $\Sigma=\sigma_{e}^{2} \mathbf{I}$; supondo que $\sigma_{u}^{2}$ e depois supondo que $\lambda=\frac{\sigma_{e}^{2}}{\sigma_{u}^{2}}$ é conhecida. Na seção 4.3 trataremos o caso em que as variâncias dos erros são heterogêneas, isto é, $\Sigma=\left(\sigma_{1}^{2}, \sigma_{2}^{2}, \ldots, \sigma_{r}^{2}\right)$, considerando-se os casos em que $\sigma_{u}^{2}$ e $\lambda_{k}=\frac{\sigma_{e_{k}}^{2}}{\sigma_{u}^{2}}$ são conhecidas, $k=1, \ldots, r$. 


\subsection{Variâncias homogêneas}

\subsubsection{Caso 1: $\sigma_{u}^{2}$ conhecida}

\section{a) Estimação dos parâmetros.}

Nesta seção estenderemos os resultados obtidos no capítulo 2 (modelos aditivos) quando é considerado o método de Nakamura (1990), o modelo de calibração "Naive"a considerar segundo a metodologia no capítulo 2 é a seguinte,

$$
\mathrm{Y}_{i}=\sum_{j=0}^{p} \beta_{j} W_{i}^{j}+\mathbf{e}_{i}
$$

onde $Y_{i}=\left(Y_{i, 1}, \ldots, Y_{i, r}\right)^{t}, \beta_{j}=\left(\beta_{j, 1}, \ldots, \beta_{j, r}\right)^{t}$ e $\mathbf{e}_{i}=\left(e_{i, 1}, \ldots, e_{i, r}\right)^{t}$ para $i=1,2, \ldots, n$ e $n$ é o total de observações, $p$ é a ordem do polinômio e $r$ é o número de instrumentos avaliados.

Quando consideramos variâncias homogêneas, uma suposição para modelos funcionais é

$$
\left(\begin{array}{c}
\mathrm{e}_{i} \\
u_{i}
\end{array}\right) \sim N_{r+1}\left[\left(\begin{array}{l}
0 \\
0
\end{array}\right),\left(\begin{array}{cc}
\Sigma & 0 \\
0 & \sigma_{u}^{2}
\end{array}\right)\right] \text { independentes, }
$$

onde $\Sigma=\sigma_{e}^{2} \mathrm{I}$, enquanto que para modelos estruturais a expressão (4.6) pode ser apresentado conjuntamente com a distribuição $X_{i} \sim N\left(\mu_{X}, \sigma_{X}^{2}\right), i=1,2, \ldots, n$, independentes. Consideramos que $\sigma_{u}^{2}$ é conhecida.

O logaritmo da função verossimilhança corrigida segundo a metodologia apresentada no capítulo 2 , seção 2.2 , é dada por uma generalização de (2.9)

$$
\begin{aligned}
\ell^{*}\left(\beta \sigma_{e}^{2} ; \mathrm{Y}, \mathrm{W}\right)= & -\frac{n r}{2} \log 2 \pi-\frac{n r}{2} \log \sigma_{e}^{2}-\frac{1}{2 \sigma_{e}^{2}} \sum_{i=1}^{n} \sum_{k=1}^{r}\left(Y_{i k}^{2}+\sum_{j=0}^{p} \beta_{j k}^{2} t_{i,(2 j)}\right. \\
& \left.-2 Y_{i k} \sum_{j=0}^{p} \beta_{j k} t_{i,(j)}+2 \sum_{\substack{j=0 \\
s<j}}^{p} \sum_{\substack{s=0 \\
s<j}}^{p} \beta_{j k} \beta_{s k} t_{i,(j+s)}\right)
\end{aligned}
$$

De acordo com (2.10) e (2.11) as funções escores corrigidos são

$$
U^{*}\left(\beta_{s k}\right)=\frac{1}{\sigma_{e}^{2}} \sum_{i=1}^{n}\left(Y_{i k} t_{i,(s)}-\sum_{j=0}^{p} \beta_{j k} t_{i,(j+s)}\right),
$$




$$
\begin{aligned}
U^{*}\left(\sigma_{e}^{2}\right)= & -\frac{n r}{2 \sigma_{e}^{2}}+\frac{1}{2 \sigma_{e}^{4}} \sum_{i=1}^{n} \sum_{k=1}^{r}\left(Y_{i k}^{2}+\sum_{j=0}^{p} \beta_{j k}^{2} t_{i,(2 j)}\right. \\
& \left.-2 Y_{i k} \sum_{j=0}^{p} \beta_{j k} t_{i,(j)}+2 \sum_{\substack{j=0 \\
j<s}}^{p} \sum_{\substack{s=0 \\
j}}^{p} \beta_{j k} \beta_{s k} t_{i,(j+s)}\right),
\end{aligned}
$$

para $s=0,1,2, \ldots, p$ e $k=1,2, \ldots, r$.

Considerando as expressões (4.8) e (4.9), podemos obter as seguintes equações lineares:

$$
\left(\begin{array}{cccc}
n & \sum_{i=1}^{n} t_{i,(1)} & \cdots & \sum_{i=1}^{n} t_{i,(p)} \\
\sum_{i=1}^{n} t_{i,(1)} & \sum_{i=1}^{n} t_{i,(2)} & \cdots & \sum_{i=1}^{n} t_{i,(p+1)} \\
\vdots & \vdots & \cdots & \vdots \\
\sum_{i=1}^{n} t_{i,(p)} & \sum_{i=1}^{n} t_{i,(p+1)} & \cdots & \sum_{i=1}^{n} t_{i,(2 p)}
\end{array}\right)\left(\begin{array}{c}
\hat{\beta}_{0, k} \\
\hat{\beta}_{1, k} \\
\vdots \\
\hat{\beta}_{p, k}
\end{array}\right)=\left(\begin{array}{c}
\sum_{i=1}^{n} Y_{i, k} \\
\sum_{i=1}^{n} Y_{i, k} t_{i,(1)} \\
\vdots \\
\sum_{i=1}^{n} Y_{i, k} t_{i,(p)}
\end{array}\right)
$$

sendo $k=1,2, \ldots, r$,

$$
\hat{\sigma}_{e}^{2}=\frac{1}{n r} \sum_{i=1}^{n} \sum_{k=1}^{m}\left(Y_{i k}^{2}+\sum_{j=0}^{p} \beta_{j k}^{2} t_{i,(2 j)}-2 Y_{i k} \sum_{j=0}^{p} \beta_{j k} t_{i,(j)}+2 \sum_{\substack{j=0 \\ j<s}}^{p} \sum_{\substack{j=0 \\ j<s}}^{p} \beta_{j k} \beta_{s k} t_{i,(j+s)}\right),
$$

para obter os valores de $t_{i,(s)}$ precisamos da equação (2.27).

Para obter a variância assintótica das estimativas necessitamos a matriz de informação corrigida dada pela seguinte expressão,

$$
I_{k, k}^{*}(\beta)=\left(\begin{array}{cccc}
n & \sum_{i=1}^{n} t_{i,(1)} & \cdots & \sum_{i=1}^{n} t_{i,(p)} \\
\sum_{i=1}^{n} t_{i,(1)} & \sum_{i=1}^{n} t_{i,(2)} & \cdots & \sum_{i=1}^{n} t_{i,(p+1)} \\
\vdots & \vdots & \cdots & \vdots \\
\sum_{i=1}^{n} t_{i,(p)} & \sum_{i=1}^{n} t_{i,(p+1)} & \cdots & \sum_{i=1}^{n} t_{i,(2 p)}
\end{array}\right),
$$

para $k=1, \ldots, r$. Da mesma maneira se precisa dos componentes das funções escores corrigidos individuais, isto é, 


$$
\mathbf{S}^{*}(\beta ; \mathrm{W}, \mathrm{Y})=\sum_{i=1}^{n} E\left[\mathrm{U}_{i}^{*}(\beta ; \mathrm{W}, \mathrm{Y}) \mathbf{U}_{i}^{*}(\beta ; \mathrm{W}, \mathrm{Y})^{t}\right]
$$

sendo que $\mathrm{U}_{i}^{*}(\beta ; \mathbf{W}, \mathbf{Y})=\left(U\left(\beta_{01}\right), \ldots, U\left(\beta_{p 1}\right), \ldots, U\left(\beta_{0 r}\right), \ldots, U\left(\beta_{p r}\right)\right)^{t}$, a finalidade disto é criar uma matriz de covariâncias de tipo sandwich, que tem a forma seguinte

$$
£^{*}(\beta ; \mathbf{W}, \mathbf{Y})=\frac{1}{n}\left(\mathbf{I}^{*}(\beta ; \mathbf{W}, \mathbf{Y})\right)^{-1} \mathbf{S}^{*}(\beta ; \mathbf{W}, \mathbf{Y})\left(\mathbf{I}^{*}(\beta ; \mathbf{W}, \mathbf{Y})\right)^{-1}
$$

com isto temos

$$
\sqrt{n}(\hat{\beta}-\beta) \stackrel{D}{\longrightarrow} N_{(p+1) \times r}\left(0, £^{*}\right) .
$$

b) Aplicação numérica.

Para esta seção consideramos um estudo da simulação. Para tal fim, consideramos as seguintes características:

1. São considerados três instrumentos a comparar em um modelo quadrático.

2. Se gera $X_{i} \sim \operatorname{Binomial}(10 ; 0,5), u_{i} \sim N(0 ; 0,05)$ e $e_{i, k} \sim N(0,1)$, sendo que $e_{i 1}, e_{i 2}, e_{i 3}$ são independentes.

3. Os parâmetros considerados na presente simulação são $\beta_{0}=(1,2,3)^{t}, \beta_{1}=(2,4,5)^{t}$ e $\beta_{2}=(3,5,9)^{t}$.

4. Considerar os modelos seguintes

$$
\begin{aligned}
& W_{i}=X_{i}+u_{i} \\
& Y_{i 1}=\beta_{01}+\beta_{11} X_{i}+\beta_{21} X_{i}^{2}+e_{i 1} \\
& Y_{i 2}=\beta_{02}+\beta_{12} X_{i}+\beta_{22} X_{i}^{2}+e_{i 2} \\
& Y_{i 3}=\beta_{03}+\beta_{13} X_{i}+\beta_{23} X_{i}^{2}+e_{i 3}
\end{aligned}
$$

\section{Resultados}

Após implementar a metodologia na linguagem Ox (Doornik, 1998) obtemos os seguintes resultados 
Tabela 4.1. Estimativas dos parâmetros segundo o método "naive"para diferentes tamanhos de amostra e Instrumento 1.

\begin{tabular}{|c|c|c|c|c|c|c|}
\hline Parâmetros & Valores & 500 & 1000 & 2000 & 3000 & 4000 \\
\hline$\beta_{0}$ & 1 & 2,36200 & 2,37080 & 2,37130 & 2,36970 & 2,36720 \\
$\beta_{1}$ & 2 & 1,17010 & 1,17390 & 1,16620 & 1,16890 & 1,17790 \\
$\beta_{2}$ & 3 & 0,08379 & 0,07107 & 0,08338 & 0,07976 & 0,08094 \\
$\sigma_{\boldsymbol{e}}^{2}$ & 1 & 7,48180 & 7,52250 & 7,50870 & 7,51220 & 7,48250 \\
\hline
\end{tabular}

Tabela 4.2. Estimativas dos parâmetros segundo o método "escore corrigido"para diferentes tamanhos de amostra e Instrumento 1.

\begin{tabular}{|c|c|c|c|c|c|c|}
\hline Parâmetros & Valores & 500 & 1000 & 2000 & 3000 & 4000 \\
\hline$\beta_{0}$ & 1 & 0,96522 & 0,92649 & 0,96984 & 1,08090 & 1,08397 \\
$\beta_{1}$ & 2 & 2,06070 & 2,03190 & 2,06490 & 1,99640 & 2,09962 \\
$\beta_{2}$ & 3 & 2,99380 & 2,95350 & 2,97690 & 3,05782 & 2,97130 \\
$\sigma_{e}^{2}$ & 1 & 0,98097 & 0,96910 & 1,02710 & 1,01055 & 0,95740 \\
\hline
\end{tabular}

Das tabelas acima podemos apreciar que os estimadores "escore corrigido"para o instrumento 1, se aproximam aos valores propostos. Gráficamente pode-se ver o seguinte 


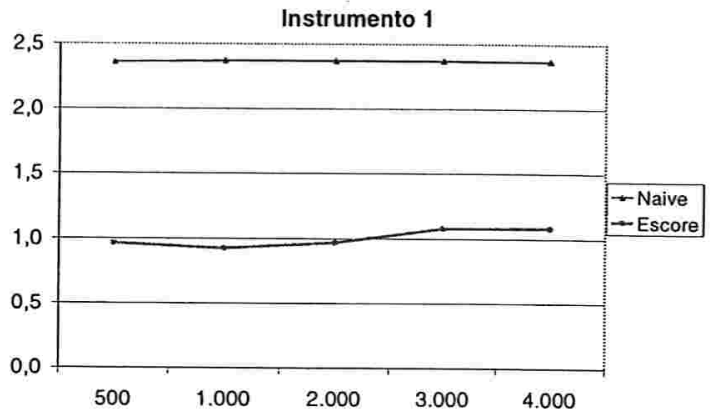

(a) Estimativa para $\beta_{0}$

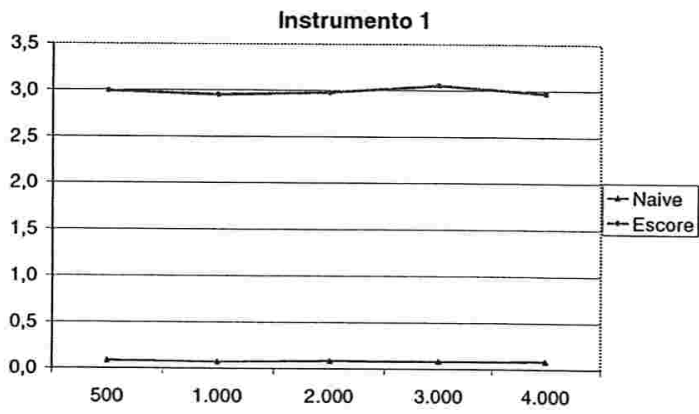

(c) Estimativa para $\beta_{2}$

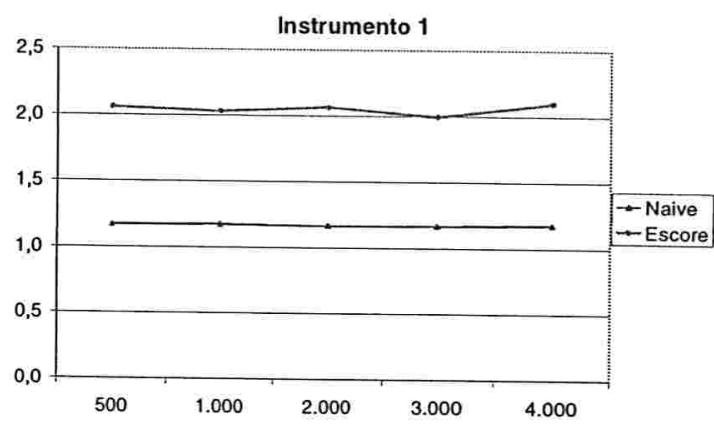

(b) Estimativa para $\beta_{1}$

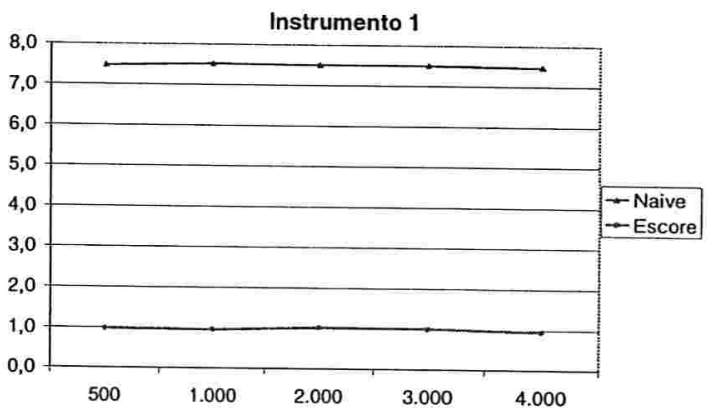

(d) Estimativa para $\sigma_{e}^{2}$

Fig. 4.1. Comportamento das estimativas "naive"e "escore corrigido"segundo o tamanho da amostra para o instrumento 1

Os gráficos acima indicam a proximidade dos estimadores "escore corrigido" a os valores propostos, para o instrumento 1. 
Tabela 4.3. Estimativas dos parâmetros segundo o método "naive"para diferentes tamanhos de amostra e Instrumento 2.

\begin{tabular}{|c|c|c|c|c|c|c|}
\hline Parâmetros & Valores & 500 & 1000 & 2000 & 3000 & 4000 \\
\hline$\beta_{0}$ & 2 & 4,52620 & 4,53620 & 4,53740 & 4,53330 & 4,52930 \\
$\beta_{1}$ & 4 & 2,12160 & 2,12340 & 2,11250 & 2,11270 & 2,13040 \\
$\beta_{2}$ & 5 & 0,13663 & 0,11978 & 0,13649 & 0,13447 & 0,13254 \\
$\sigma_{e}^{2}$ & 1 & 7,48180 & 7,52250 & 7,50870 & 7,51220 & 7,48250 \\
\hline
\end{tabular}

Tabela 4.4. Estimativas dos parâmetros segundo o método "escore corrigido"para diferentes tamanhos de amostra e Instrumento 2.

\begin{tabular}{|c|c|c|c|c|c|c|}
\hline Parâmetros & Valores & 500 & 1000 & 2000 & 3000 & 4000 \\
\hline$\beta_{0}$ & 2 & 1,96250 & 1,87390 & 1,93740 & 2,14990 & 2,01830 \\
$\beta_{1}$ & 4 & 4,07850 & 4,04490 & 4,03090 & 4,00560 & 3,94370 \\
$\beta_{2}$ & 5 & 4,93360 & 4,98710 & 4,97270 & 5,05210 & 5,07090 \\
$\sigma_{e}^{2}$ & 1 & 0,98097 & 0,96910 & 1,02710 & 1,01055 & 0,95740 \\
\hline
\end{tabular}

Para o instrumento 2 vemos que os estimadores de "escore corrigido" se aproximam a os valores fixados na simulação que os estimadores "naive", gráficamente este comportamento se expressa nas figuras seguintes, 


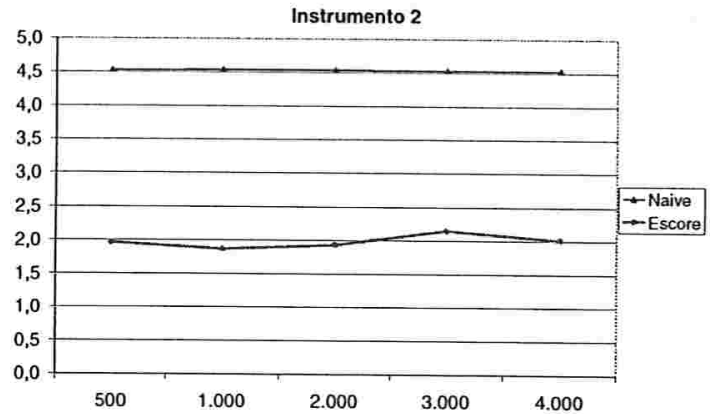

(a) Estimativa para $\beta_{0}$

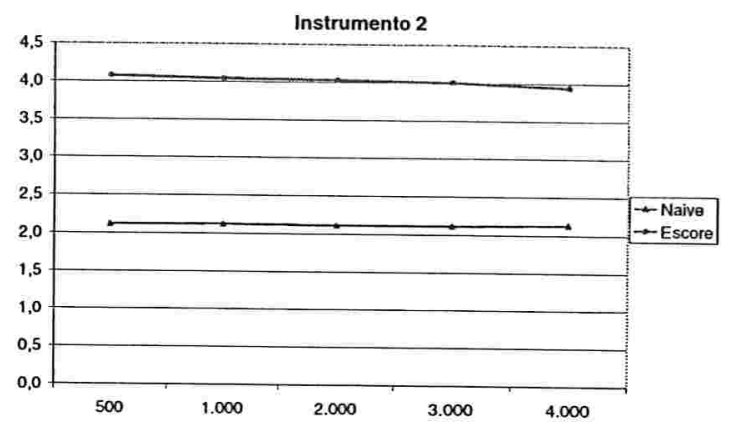

(b) Estimativa para $\beta_{1}$

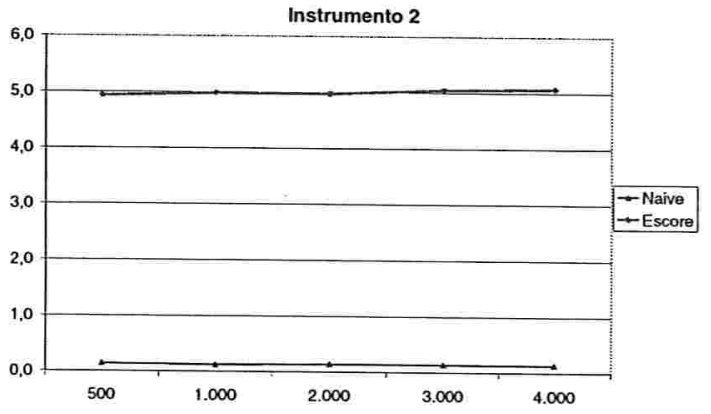

(c) Estimativa para $\beta_{2}$

Fig. 4.2. Comportamento das estimativas "naive"e "escore corrigido"segundo o tamanho da amostra e instrumento 2

Pode-se observar dos gráficos acima que as estimativas "escore corrigido" se aproximam aos valores fixados na simulação quando é considerado o instrumento 2 . 
Tabela 4.5. Estimativas dos parâmetros segundo o método "naive"para diferentes tamanhos de amostra e Instrumento 3.

\begin{tabular}{|c|c|c|c|c|c|c|}
\hline Parâmetros & Valores & 500 & 1000 & 2000 & 3000 & 4000 \\
\hline$\beta_{0}$ & 3 & 6,72790 & 6,73980 & 6,74160 & 6,73460 & 6,72860 \\
$\beta_{1}$ & 5 & 3,27410 & 3,27120 & 3,25660 & 3,25320 & 3,28120 \\
$\beta_{2}$ & 9 & 0,24048 & 0,21995 & 0,24195 & 0,24186 & 0,23690 \\
$\sigma_{e}^{2}$ & 1 & 7,48180 & 7,52250 & 7,50870 & 7,51220 & 7,48250 \\
\hline
\end{tabular}

Tabela 4.6. Estimativas dos parâmetros segundo o método "escore corrigido"para diferentes tamanhos de amostra e Instrumento 3.

\begin{tabular}{|c|c|c|c|c|c|c|}
\hline Parâmetro & Valor & 500 & 1000 & 2000 & 3000 & 4000 \\
\hline$\beta_{0}$ & 3 & 2,96250 & 3,07390 & 2,98740 & 3,04990 & 3,01830 \\
$\beta_{1}$ & 5 & 4,97850 & 4,98490 & 4,99090 & 5,00560 & 5,04370 \\
$\beta_{2}$ & 9 & 8,93360 & 8,98710 & 8,99270 & 9,05210 & 9,07090 \\
$\sigma_{e}^{2}$ & 1 & 0,98097 & 0,96910 & 1,02710 & 1,01055 & 0,95740 \\
\hline
\end{tabular}

Da mesma maneira no instrumento 3 , as estimativas no método "naive" não se aproximam a os valores fixados na simulação, enquanto os estimadores "escore corrigido" se aproximam bastante aos valores fixados. 


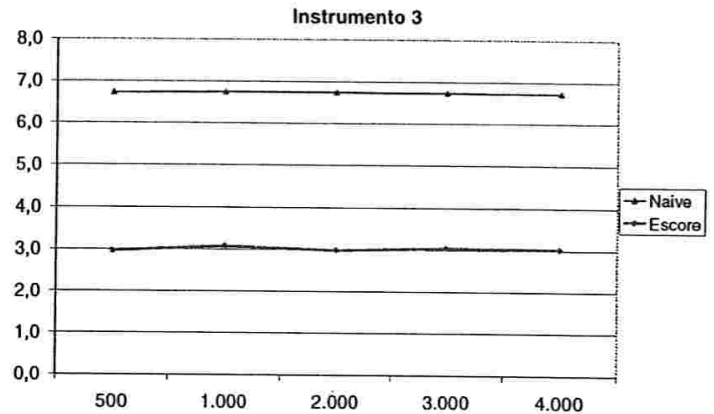

(a) Estimativa para $\beta_{0}$

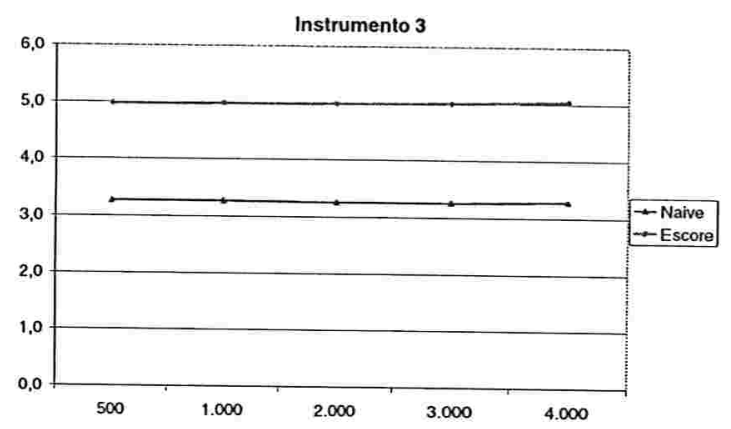

(b) Estimativa para $\beta_{1}$

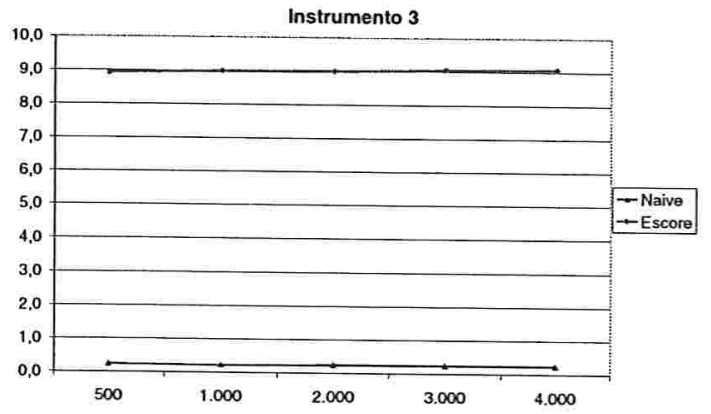

(c) Estimativa para $\beta_{2}$

Fig. 4.3. Comportamento das estimativas "naive"e "escore corrigido"segundo o tamanho da amostra

Esta situação pode ser vistos nos gráficos acima, observando-se no caso dos estimadores "escore corrigido" a proximidade com os valores fixados na simulação. 


\subsection{Caso 2: $\lambda=\frac{\sigma_{e}^{2}}{\sigma_{u}^{2}}$ conhecida}

\section{a) Estimação dos parâmetros.}

Nesta seção aplicaremos a metodologia do escore corrigido apresentada por Bolfarine e Gimenez (1997) aos modelos polinomiais para calibração comparativa. Para tal fim, utilizaremos os procedimentos vistos na seção 2.3 .

O modelo de calibração "naive"é como na equação (4.5). Quando consideramos variâncias homogêneas, a suposição para modelos de tipo funcionais é

$$
\left(\begin{array}{l}
\mathrm{e}_{i} \\
u_{i}
\end{array}\right) \sim N_{r+1}\left[\left(\begin{array}{l}
0 \\
0
\end{array}\right),\left(\begin{array}{cc}
\lambda \sigma_{u}^{2} \mathrm{I} & 0 \\
0 & \sigma_{u}^{2}
\end{array}\right)\right] \text {, independentes, } i=1, \ldots, n,
$$

enquanto nos modelos estruturais a expressão (4.10) pode ser apresentada conjuntamente com a distribuição $X_{i} \sim N\left(\mu_{X}, \sigma_{X}^{2}\right)$, independentes, $i=1,2, \ldots, n$. Consideramos que $\Lambda$ é conhecida.

Para a estimação dos parâmetros consideramos um processo algorítmico de tipo Newton-Raphson, que segue a seguinte estrutura,

\section{Algoritmo para estimação dos parâmetros no caso de Escore Corrigida}

1. Atribua um valor inicial para $\sigma_{u}^{2}$.

2. Substitua $\sigma_{u}^{2}$ da etapa anterior na expressão seguinte:

$$
\left(\begin{array}{cccc}
n & \sum_{i=1}^{n} t_{i,(1)} & \cdots & \sum_{i=1}^{n} t_{i,(p)} \\
\sum_{i=1}^{n} t_{i,(1)} & \sum_{i=1}^{n} t_{i,(2)} & \cdots & \sum_{i=1}^{n} t_{i,(p+1)} \\
\vdots & \vdots & \cdots & \vdots \\
\sum_{i=1}^{n} t_{i,(p)} & \sum_{i=1}^{n} t_{i,(p+1)} & \cdots & \sum_{i=1}^{n} t_{i,(2 p)}
\end{array}\right)\left(\begin{array}{c}
\hat{\beta}_{0, k}^{(m)} \\
\hat{\beta}_{1, k}^{(m)} \\
\vdots \\
\hat{\beta}_{p, k}^{(m)}
\end{array}\right)=\left(\begin{array}{c}
\sum_{i=1}^{n} Y_{i, k} \\
\sum_{i=1}^{n} Y_{i, k} t_{i,(1)} \\
\vdots \\
\sum_{i=1}^{n} Y_{i, k} t_{i,(p)}
\end{array}\right)
$$

para $k=1, \ldots, r$, lembrando que cada valor de $t_{i,(s)}$ segue a forma da expressão (2.27). 
3. Agora com os valores $\beta_{j, k}^{(m)}, j=0,1, \ldots, p$ e $k=1,2, \ldots, r$ obtidos devemos atualizar $\sigma_{u}^{2}$. Para isto consideramos o método de Newton-Raphson em cada instrumento que segue segundo,

$$
\sigma_{u}^{2(m+1)}=\sigma_{u}^{2(m)}+\frac{U^{*}\left(\sigma_{u}^{2(m)}\right)}{I^{*}\left(\sigma_{u}^{2(m)}\right)}
$$

\section{b) Aplicação numérica.}

Nesta seção apresentamos uma simulação com as seguintes características:

1. São considerados três instrumentos a comparar em um modelo quadrático.

2. Se gera $X_{i} \sim \operatorname{Binomial}(10 ; 0,5), u_{i} \sim N(0 ; 0,05), \lambda=20$ e $e_{i, k} \sim N\left(0 ; \lambda \times \sigma_{u}^{2}\right)$, sendo que $e_{i 1}, e_{i 2}, e_{i 3}$ são independentes.

3. Os parâmetros considerados na presente simulação são $\beta_{0}=(1,2,3)^{t}, \beta_{1}=(2,4,5)^{t} \mathrm{e}$ $\beta_{2}=(3,5,9)^{t}$.

4. Considerar os modelos seguintes

$$
\begin{aligned}
& W_{i}=X_{i}+u_{i} \\
& Y_{i 1}=\beta_{01}+\beta_{11} X_{i}+\beta_{21} X_{i}^{2}+e_{i 1} \\
& Y_{i 2}=\beta_{02}+\beta_{12} X_{i}+\beta_{22} X_{i}^{2}+e_{i 2} \\
& Y_{i 3}=\beta_{03}+\beta_{13} X_{i}+\beta_{23} X_{i}^{2}+e_{i 3}
\end{aligned}
$$

\section{Resultados}

Após implementada a metodologia na linguagem Ox (Doornik, 1998), os resultados obtidos são apresentados na tabela a seguir 
Tabela 4.7. Estimativas dos parâmetros segundo o método "Naive" para diferentes tamanhos de amostra e Instrumento 1.

\begin{tabular}{|c|c|c|c|c|c|c|}
\hline Parâmetros & Valor & 500 & 1000 & 2000 & 3000 & 4000 \\
\hline$\beta_{0}$ & 1 & 1,53590 & 1,54290 & 1,54300 & 1,54360 & 1,54290 \\
$\beta_{1}$ & 2 & 0,26781 & 0,27655 & 0,27158 & 0,27732 & 0,27851 \\
$\beta_{2}$ & -1 & $-0,01945$ & $-0,03002$ & $-0,02182$ & $-0,02696$ & $-0,02380$ \\
$\sigma_{e}^{2}$ & 1 & 1,22900 & 1,23130 & 1,23430 & 1,23810 & 1,23840 \\
\hline
\end{tabular}

Tabela 4.8. Estimativas dos parâmetros segundo o método "escore corrigido" para diferentes tamanhos de amostra e Instrumento 1.

\begin{tabular}{|c|c|c|c|c|c|c|}
\hline Parâmetros & Valor & 500 & 1000 & 2000 & 3000 & 4000 \\
\hline$\beta_{0}$ & 1 & 1,00090 & 0,95853 & 0,98217 & 1,01940 & 1,01510 \\
$\beta_{1}$ & 2 & 1,96910 & 2,08120 & 2,03050 & 1,98990 & 1,99350 \\
$\beta_{2}$ & -1 & $-0,97667$ & $-1,06360$ & $-1,01070$ & $-0,99413$ & $-0,99264$ \\
$\sigma_{e}^{2}$ & 1 & 0,91652 & 0,97094 & 0,99219 & 0,99275 & 1,01805 \\
\hline
\end{tabular}

Das tabelas acima podemos apreciar que as estimativas do método "escore corrigido" apresentam uma maior proximidade aos valores fixados que o método naive. 


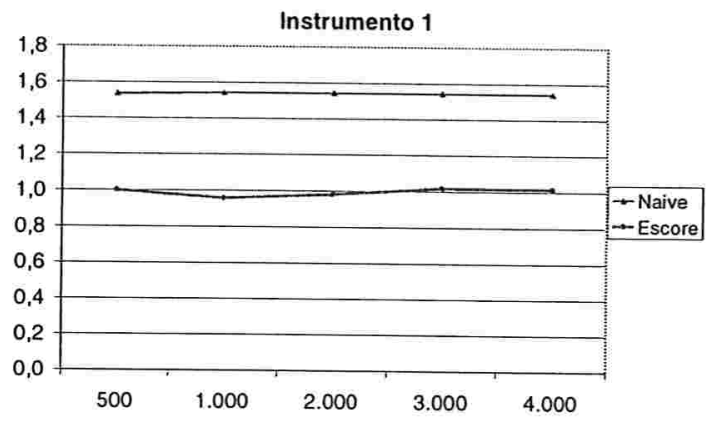

(a) Estimativa para $\beta_{0}$

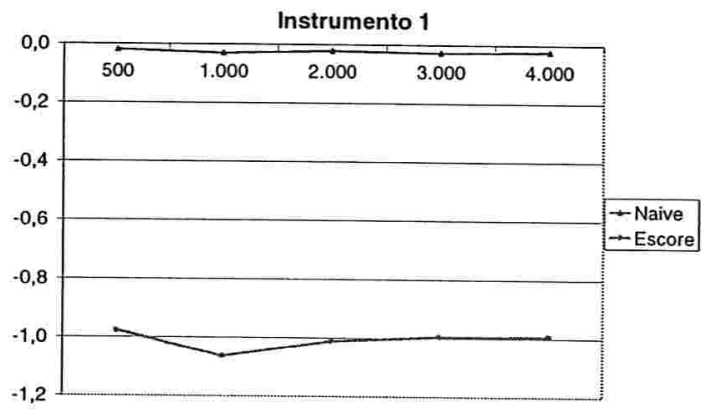

(c) Estimativa para $\beta_{2}$

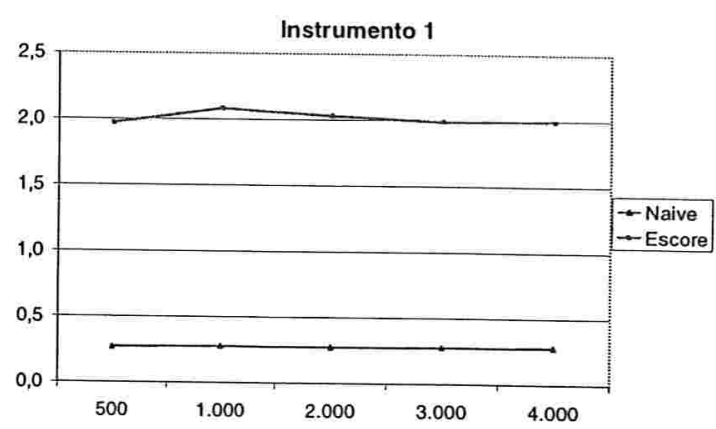

(b) Estimativa para $\beta_{1}$

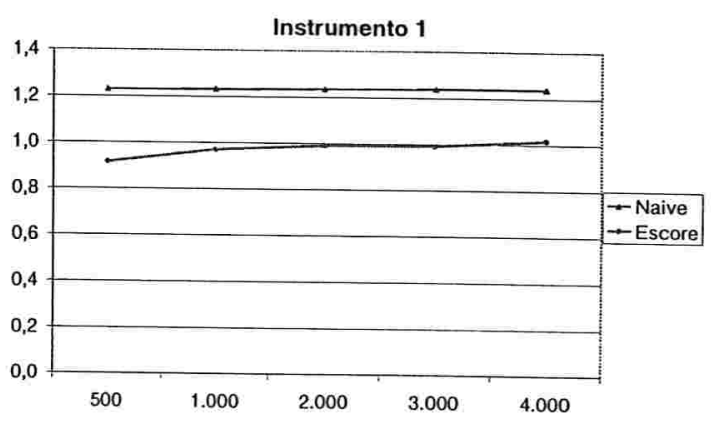

(d) Estimativa para $\sigma_{e}^{2}$

Fig. 4.4. Comportamento das estimativas "naive"e "escore corrigido"segundo o tamanho da amostra no instrumento 1

Os gráficos acima amostram o bom ajuste das estimativas do método "escore corrigido" no instrumento 1 aos valores fixados na simulação. 
Tabela 4.9. Estimativas dos parâmetros segundo o método "naive"para diferentes tamanhos de amostra e Instrumento 2.

\begin{tabular}{|c|c|c|c|c|c|c|}
\hline Parâmetros & Valor & 500 & 1000 & 2000 & 3000 & 4000 \\
\hline$\beta_{0}$ & 2 & 2,22870 & 2,33640 & 2,23630 & 2,13730 & 2,23620 \\
$\beta_{1}$ & -1 & 0,19393 & 0,19832 & 0,19409 & 0,19904 & 0,19893 \\
$\beta_{2}$ & 2 & 0,05616 & 0,04856 & 0,05630 & 0,05106 & 0,05590 \\
$\sigma_{\boldsymbol{e}}^{2}$ & 1 & 1,22900 & 1,23130 & 1,23430 & 1,23810 & 1,23840 \\
\hline
\end{tabular}

Tabela 4.10. Estimativas dos parâmetros segundo o método "escore corrigido"para diferentes tamanhos de amostra e Instrumento 2.

\begin{tabular}{|c|c|c|c|c|c|c|}
\hline Parâmetros & Valor & 500 & 1000 & 2000 & 3000 & 4000 \\
\hline$\beta_{0}$ & 2 & 1,98150 & 1,98940 & 2,04230 & 2,00810 & 1,98410 \\
$\beta_{1}$ & -1 & $-0,97018$ & $-0,98095$ & $-1,01690$ & $-1,08420$ & $-1,07879$ \\
$\beta_{2}$ & 2 & 1,96940 & 1,96170 & 2,02070 & 2,01036 & 1,97380 \\
$\sigma_{\boldsymbol{e}}^{2}$ & 1 & 0,91652 & 0,97094 & 0,99219 & 0,99275 & 1,01805 \\
\hline
\end{tabular}

Para o instrumento 2 pode-se observar que as estimativas do método "escore corrigido" tem uma boa proximidade aos valores fixados na simulação, gráficamente este comportamento se expressa nas figuras seguintes, 


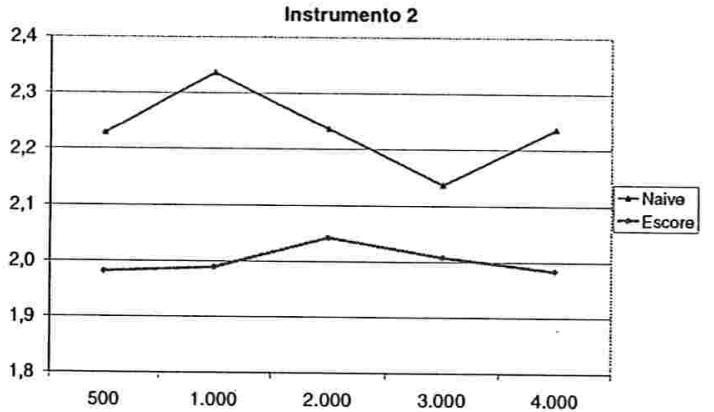

(a) Estimativa para $\beta_{0}$

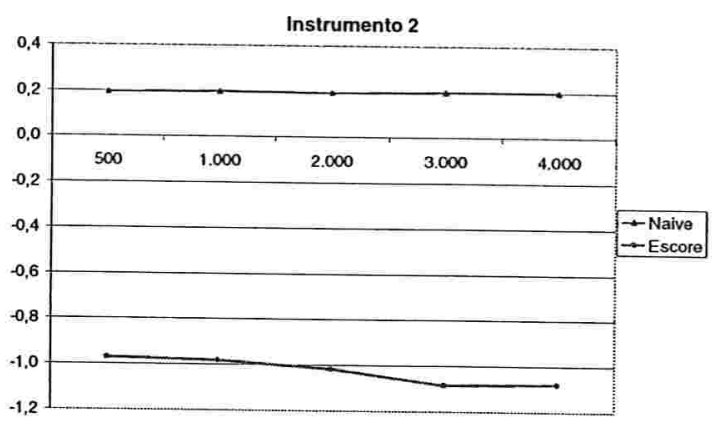

(b) Estimativa para $\beta_{1}$

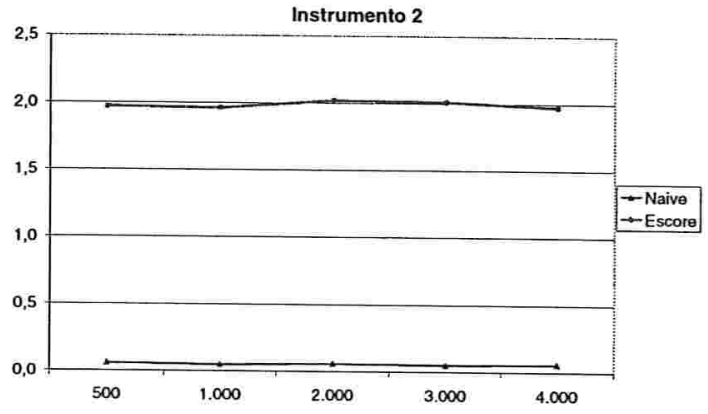

(c) Estimativa para $\beta_{2}$

Fig. 4.5. Comportamento das estimativas "naive"e "escore corrigido"segundo o tamanho da amostra no instrumento 2

Das figuras acima pode-se observar que as estimativas do método "naive" não se aproximam aos valores fixados na simulação quando é avaliado no instrumento 2, como se mencionou acima o método "escore corrigido" apresenta estimativas bastante próximas aos valores fixados. 
Tabela 4.11. Estimativas dos parâmetros segundo o método "naive"para diferentes tamanhos de amostra e Instrumento 3.

\begin{tabular}{|c|c|c|c|c|c|c|}
\hline Parâmetros & Valor & 500 & 1000 & 2000 & 3000 & 4000 \\
\hline$\beta_{0}$ & -1 & $-0,05106$ & $-0,04314$ & $-0,04288$ & $-0,04335$ & $-0,04492$ \\
$\beta_{1}$ & 2 & 0,71895 & 0,72522 & 0,71889 & 0,72311 & 0,72822 \\
$\beta_{2}$ & 1 & 0,03217 & 0,02053 & 0,03078 & 0,02640 & 0,02857 \\
$\sigma_{e}^{2}$ & 1 & 1,22900 & 1,23130 & 1,23430 & 1,23810 & 1,23840 \\
\hline
\end{tabular}

Tabela 4.12. Estimativas dos parâmetros segundo o método "escore corrigido"para diferentes tamanhos de amostra e Instrumento 3.

\begin{tabular}{|c|c|c|c|c|c|c|}
\hline Parâmetros & Valor & 500 & 1000 & 2000 & 3000 & 4000 \\
\hline$\beta_{0}$ & -1 & $-1,01690$ & $-1,05750$ & $-1,05400$ & $-0,99983$ & $-1,07260$ \\
$\beta_{1}$ & 2 & 2,01490 & 2,05010 & 2,04770 & 1,97320 & 2,04980 \\
$\beta_{2}$ & 1 & 1,05857 & 1,09497 & 1,06310 & 1,04200 & 1,05932 \\
$\sigma_{e}^{2}$ & 1 & 0,91652 & 0,97094 & 0,99219 & 0,99275 & 1,01805 \\
\hline
\end{tabular}

Da mesma maneira no instrumento 3, os estimadores "naive" estabilizam seus valores longe dos parâmetros propostos, enquanto os estimadores de "escore corrigido" tem uma boa aproximação aos valores propostos na simulação. 


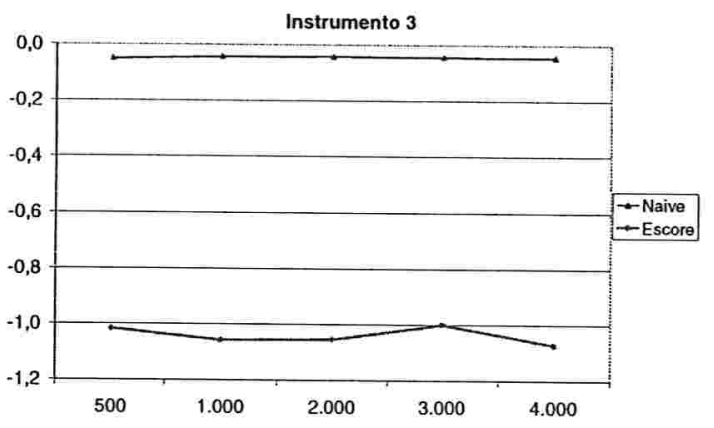

(a) Estimativa para $\beta_{0}$

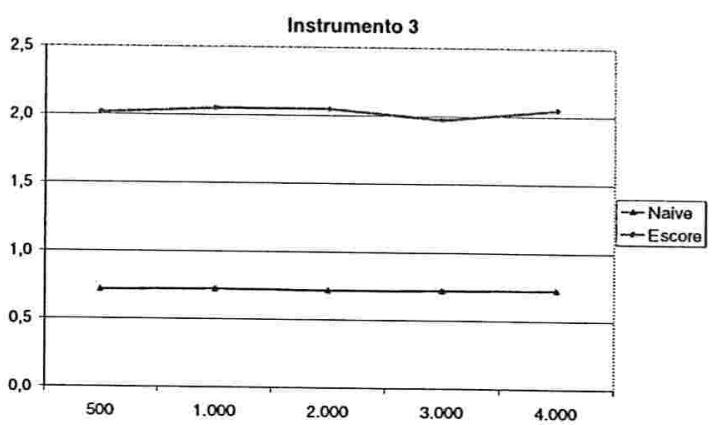

(b) Estimativa para $\beta_{1}$

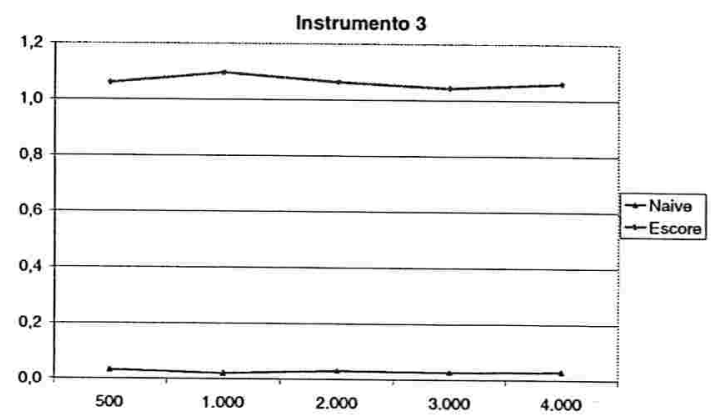

(c) Estimativa para $\beta_{2}$

Fig. 4.6. Comportamento das estimativas "naive"e "escore corrigido"segundo o tamanho da amostra no instrumento 3

Nos gráficos da acima, pode-se observar que no caso das estimativas do método de "escore corrigido"estas foram bastante próximas aos valores fixados, enquanto que as estimativas do método "naive" estas estão afastados dos valores fixados na simulação para o instrumento 3 . 


\subsection{Variâncias heterogêneas}

\subsubsection{Caso 1: $\sigma_{u}^{2}$ conhecida}

\section{a) Estimação dos parâmetros.}

Nesta seção estenderemos os resultados obtidos no capítulo 2 (modelos aditivos) quando é considerado o método de Nakamura (1990), o modelo de calibração "naive"a considerar segundo a metodologia no capítulo 2 é a mesma apresentada na equação (4.5), quando consideramos variâncias heterogêneas a suposição para modelos de tipo funcional é

$$
\left(\begin{array}{l}
\mathrm{e}_{i} \\
u_{i}
\end{array}\right) \sim N_{r+1}\left[\left(\begin{array}{l}
0 \\
0
\end{array}\right),\left(\begin{array}{cc}
\Sigma_{e e} & 0 \\
0 & \sigma_{u}^{2}
\end{array}\right)\right],
$$

onde $\Sigma_{e e}=\left(\sigma_{1}^{2}, \sigma_{2}^{2}, \ldots, \sigma_{r}^{2}\right)$, enquanto para modelos de tipo estrutural a expressão (4.11) pode ser apresentado conjuntamente com a distribuição $X_{i} \sim N\left(\mu_{X}, \sigma_{X}^{2}\right), i=1,2, \ldots, n$. Consideramos que $\sigma_{u}^{2}$ é conhecida.

O logaritmo da função de verossimilhança corrigida segundo a metodologia apresentada no capítulo 2 , seção 2.2 , é dada por:

$$
\begin{aligned}
\ell^{*}\left(\beta, \sigma_{e}^{2} ; \mathrm{Y}, \mathrm{W}\right)= & -\frac{n r}{2} \log 2 \pi-\frac{n}{2} \sum_{k=1}^{r} \log \sigma_{e}^{2}-\frac{1}{2 \sigma_{e}^{2}} \sum_{k=1}^{r} \frac{1}{2 \sigma_{k}^{2}} \sum_{i=1}^{n}\left(Y_{i k}^{2}+\sum_{j=0}^{p} \beta_{j k}^{2} t_{i,(2 j)}\right. \\
& \left.-2 Y_{i k} \sum_{j=0}^{p} \beta_{j k} t_{i,(j)}+2 \sum_{\substack{j=0 \\
j<s}}^{p} \sum_{\substack{s=0 \\
j<s}}^{p} \beta_{j k} \beta_{s k} t_{i,(j+s)}\right) .
\end{aligned}
$$

Sendo as funções escore corrigidas dadas por

$$
\begin{aligned}
U^{*}\left(\beta_{s k}\right)= & \frac{1}{\sigma_{k}^{2}} \sum_{i=1}^{n}\left(Y_{i k} t_{i,(s)}-\sum_{j=0}^{p} \beta_{j k} t_{i,(j+s)}\right), \\
U^{*}\left(\sigma_{k}^{2}\right)= & -\frac{n}{2 \sigma_{k}^{2}}+\frac{1}{2 \sigma_{k}^{4}} \sum_{i=1}^{n}\left(Y_{i k}^{2}+\sum_{j=0}^{p} \beta_{j k}^{2} t_{i,(2 j)}\right. \\
& \left.-2 Y_{i k} \sum_{j=0}^{p} \beta_{j k} t_{i,(j)}+2 \sum_{j=0}^{p} \sum_{\substack{s=0 \\
j<s}}^{p} \beta_{j k} \beta_{s k} t_{i,(j+s)}\right),
\end{aligned}
$$


para $s=0,1,2, \ldots, p$ parâmetros e $k=1,2, \ldots, r$ instrumentos.

Considerando as expressões (4.13) e (4.14) podemos obter as seguintes equações lineares,

$$
\left(\begin{array}{cccc}
n & \sum_{i=1}^{n} t_{i,(1)} & \cdots & \sum_{i=1}^{n} t_{i,(p)} \\
\sum_{i=1}^{n} t_{i,(1)} & \sum_{i=1}^{n} t_{i,(2)} & \cdots & \sum_{i=1}^{n} t_{i,(p+1)} \\
\vdots & \vdots & \cdots & \vdots \\
\sum_{i=1}^{n} t_{i,(p)} & \sum_{i=1}^{n} t_{i,(p+1)} & \cdots & \sum_{i=1}^{n} t_{i,(2 p)}
\end{array}\right)\left(\begin{array}{c}
\hat{\beta}_{0, k} \\
\hat{\beta}_{1, k} \\
\vdots \\
\hat{\beta}_{p, k}
\end{array}\right)=\left(\begin{array}{c}
\sum_{i=1}^{n} Y_{i, k} \\
\sum_{i=1}^{n} Y_{i, k} t_{i,(1)} \\
\vdots \\
\sum_{i=1}^{n} Y_{i, k} t_{i,(p)}
\end{array}\right)
$$

e

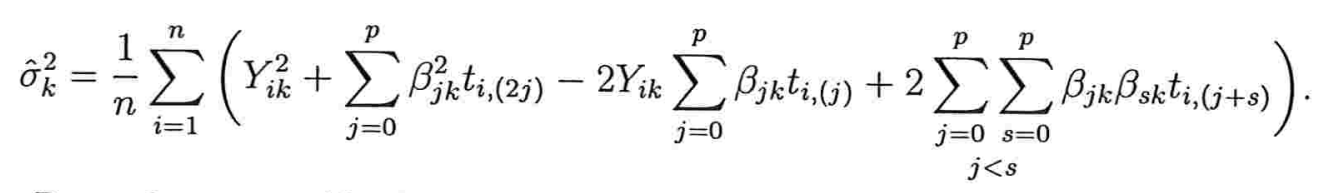

Para obter a variância assintótica necessitamos a matriz de informação corrigida dada pela seguinte expressão,

$$
I^{*}(\beta)=\left(\begin{array}{cccc}
n & \sum_{i=1}^{n} t_{i,(1)} & \cdots & \sum_{i=1}^{n} t_{i,(p)} \\
\sum_{i=1}^{n} t_{i,(1)} & \sum_{i=1}^{n} t_{i,(2)} & \cdots & \sum_{i=1}^{n} t_{i,(p+1)} \\
\vdots & \vdots & \ldots & \vdots \\
\sum_{i=1}^{n} t_{i,(p)} & \sum_{i=1}^{n} t_{i,(p+1)} & \cdots & \sum_{i=1}^{n} t_{i,(2 p)}
\end{array}\right),
$$

assim como um componente que é o produto das funções escores individuais, isto é,

$$
\mathbf{S}^{*}(\beta ; \mathbf{W}, \mathbf{Y})=E\left[\sum_{i=1}^{n} \mathbf{U}_{i}^{*}(\beta ; \mathbf{W}, \mathbf{Y}) \mathbf{U}_{i}^{*}(\beta ; \mathbf{W}, \mathbf{Y})^{t}\right]
$$

com a finalidade de criar uma matriz de covariâncias de tipo sandwich, que segue a continuação

$$
£^{*}(\beta ; \mathbf{W}, \mathbf{Y})=\frac{1}{n}\left(\mathbf{I}^{*}(\beta ; \mathbf{W}, \mathbf{Y})\right)^{-1} \mathbf{S}^{*}(\beta ; \mathbf{W}, \mathbf{Y})\left(\mathbf{I}^{*}(\beta ; \mathbf{W}, \mathbf{Y})\right)^{-1}
$$

e desta forma temos

$$
\sqrt{n}(\hat{\beta}-\beta) \stackrel{D}{\longrightarrow} N_{p+1}\left(0, £^{*}\right) .
$$




\section{b) Aplicação numérica.}

Para esta seção consideramos uma simulação com as seguintes características:

1. São considerados três instrumentos a comparar em um modelo quadrático.

2. Se gera $X_{i} \sim \operatorname{Binomial}(10 ; 0,5), u_{i} \sim N(0 ; 0,25)$ e $e_{1} \sim N(0 ; 0,25), e_{2} \sim N(0 ; 0,5)$, $e_{3} \sim N(0 ; 1)$, sendo que $e_{i 1}, e_{i 2}, e_{i 3}$ são independentes.

3. Os parâmetros considerados na presente simulação são $\beta_{0}=(-1,1,1)^{t}, \beta_{1}=(1,-1,4)^{t}$ e $\beta_{2}=(1,1,-1)^{t}$

4. Considerar os modelos seguintes

$$
\begin{aligned}
& W_{i}=X_{i}+u_{i} \\
& Y_{i 1}=\beta_{01}+\beta_{11} X_{i}+\beta_{21} X_{i}^{2}+e_{i 1} \\
& Y_{i 2}=\beta_{02}+\beta_{12} X_{i}+\beta_{22} X_{i}^{2}+e_{i 2} \\
& Y_{i 3}=\beta_{03}+\beta_{13} X_{i}+\beta_{23} X_{i}^{2}+e_{i 3}
\end{aligned}
$$

\section{Resultados}

Tabela 4.13. Estimativas dos parâmetros segundo o modelo "naive" para diferentes tamanhos de amostra e Instrumento 1.

\begin{tabular}{|c|c|c|c|c|c|c|}
\hline Parâmetros & Valor & 500 & 1000 & 2000 & 3000 & 4000 \\
\hline$\beta_{0}$ & -1 & $-0,42323$ & $-0,42130$ & $-0,41563$ & $-0,42186$ & $-0,41796$ \\
$\beta_{1}$ & 1 & 0,48097 & 0,46838 & 0,47222 & 0,47623 & 0,47811 \\
$\beta_{2}$ & 1 & 0,02691 & 0,03154 & 0,02539 & 0,02622 & 0,02165 \\
$\sigma_{e}^{2}$ & 0,25 & 0,50216 & 0,50369 & 0,50594 & 0,50309 & 0,50484 \\
\hline
\end{tabular}


Tabela 4.14. Estimativas dos parâmetros segundo o modelo "escore corrigido"para diferentes tamanhos de amostra e Instrumento 1.

\begin{tabular}{|c|c|c|c|c|c|c|}
\hline Parâmetros & Valor & 500 & 1000 & 2000 & 3000 & 4000 \\
\hline$\beta_{0}$ & -1 & $-1,05530$ & $-1,06730$ & $-0,99374$ & $-1,01100$ & $-1,05440$ \\
$\beta_{1}$ & 1 & 1,99530 & 1,90610 & 1,98581 & 1,93840 & 1,98610 \\
$\beta_{2}$ & 1 & 0,97258 & 1,03663 & 1,05490 & 0,99640 & 0,97847 \\
$\sigma_{e}^{2}$ & 0,25 & 0,28396 & 0,22261 & 0,23737 & 0,24797 & 0,25169 \\
\hline
\end{tabular}

Das tabelas acima podemos apreciar que os estimadores "escore corrigido" estão próximos aos valores fixados na presente simulação quando é avaliado o instrumento 1 .

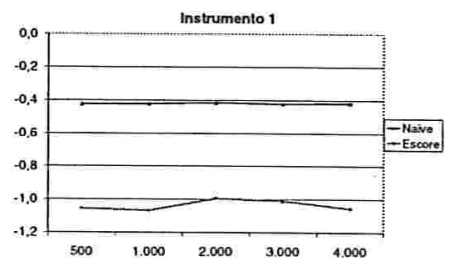

(a) Estimativa para $\beta_{0}$

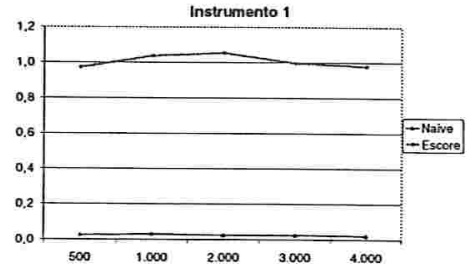

(c) Estimativa para $\beta_{2}$

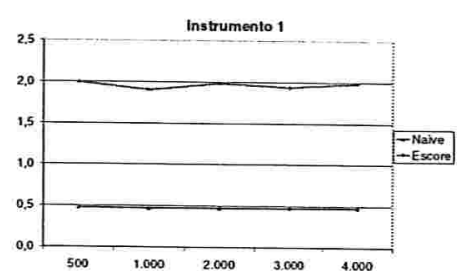

(b) Estimativa para $\beta_{1}$

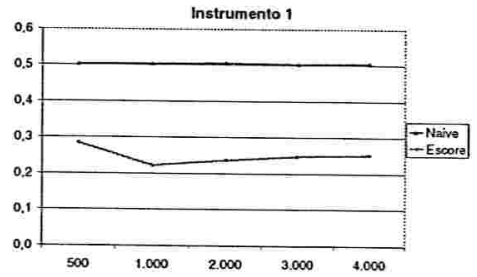

(d) Estimativa para $\beta_{2}$

Fig. 4.7. Comportamento das estimativas "naive"e "escore corrigido"segundo o tamanho da amostra no instrumento 1 
Dos gráficos acima pode-se apreciar que as estimativas do método do "escore corrigido" estão próximos aos valores fixados, enquanto as estimativas do método "naive" não.

Tabela 4.15. Estimativas dos parâmetros segundo o modelo "naive"para diferentes tamanhos de amostra e Instrumento 2 .

\begin{tabular}{|c|c|c|c|c|c|c|}
\hline Parâmetros & Valor & 500 & 1000 & 2000 & 3000 & 4000 \\
\hline$\beta_{0}$ & 1 & 0,82958 & 0,83453 & 0,82787 & 0,83237 & 0,83238 \\
$\beta_{1}$ & -1 & $-0,033512$ & $-0,0224$ & $-0,026124$ & $-0,023105$ & $-0,027958$ \\
$\beta_{2}$ & 1 & 0,028706 & $2,05 \mathrm{E}-02$ & 0,023947 & 0,024398 & 0,026684 \\
$\sigma_{e}^{2}$ & 0,5 & 0,50292 & 0,50645 & 0,50399 & 0,50546 & 0,50554 \\
\hline
\end{tabular}

Tabela 4.16. Estimativas dos parâmetros segundo o modelo "escore corrigido"para diferentes tamanhos de amostra e Instrumento 2.

\begin{tabular}{|c|c|c|c|c|c|c|}
\hline Parâmetros & Valor & 500 & 1000 & 2000 & 3000 & 4000 \\
\hline$\beta_{0}$ & 1 & 1,04590 & 1,00250 & 0,98508 & 1,00480 & 0,99910 \\
$\beta_{1}$ & -1 & $-1,26230$ & $-0,93013$ & $-1,00440$ & $-1,02650$ & $-0,96270$ \\
$\beta_{2}$ & 1 & 1,23640 & 0,90000 & 1,03700 & 1,02980 & 0,94483 \\
$\sigma_{e}^{2}$ & 0,5 & 0,51940 & 0,50710 & 0,50370 & 0,51250 & 0,49838 \\
\hline
\end{tabular}

Para o instrumento 2 observa-se que as estimativas do método do "escore corrigido" estão mais próximos aos valores fixados na simulação que as estimativas do método "naive", gráficamente este comportamento se expressa nas figuras seguintes, 


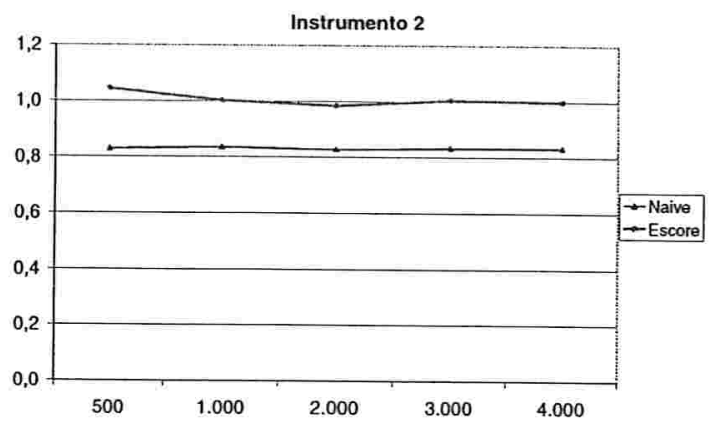

(a) Estimativa para $\beta_{0}$

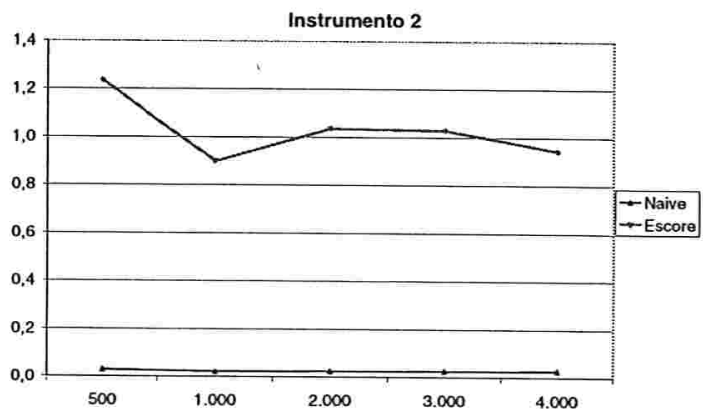

(c) Estimativa para $\beta_{2}$

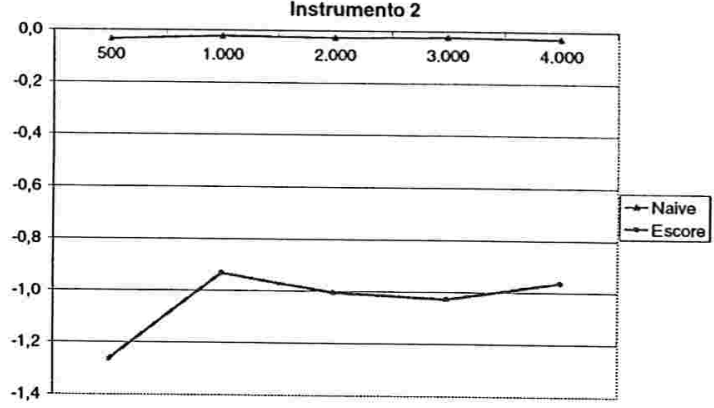

(b) Estimativa para $\beta_{1}$

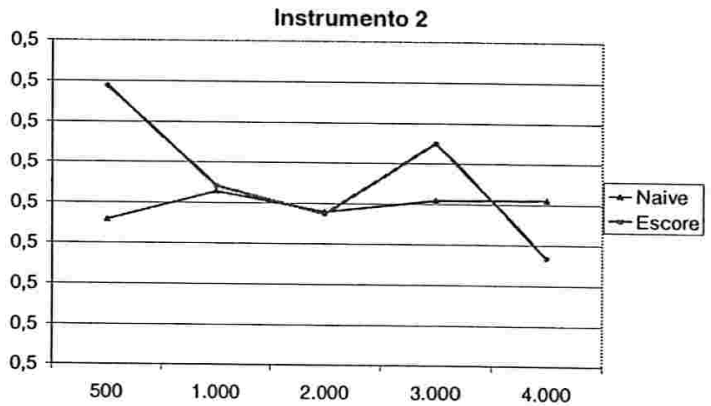

(d) Estimativa para $\beta_{2}$

Fig. 4.8. Comportamento das estimativas "naive"e "escore corrigido"segundo o tamanho da amostra no instrumento 2

A partir destas figuras pode-se observar que para o instrumento 2 as estimativas do método "naive" não conseguem aproximar-se aos valores fixados, enquanto as estimativas do método do "escore corrigido" conseguem aproximar-se aos valores fixados. 
Tabela 4.17. Estimativas dos parâmetros segundo o modelo "naive" para diferentes tamanhos de amostra e Instrumento 3.

\begin{tabular}{|c|c|c|c|c|c|c|}
\hline Parâmetros & Valor & 500 & 1000 & 2000 & 3000 & 4000 \\
\hline$\beta_{0}$ & 1 & 2,28960 & 2,29520 & 2,29070 & 2,29040 & 2,29420 \\
$\beta_{1}$ & 4 & 0,79817 & 0,77016 & 0,78311 & 0,77741 & 0,77530 \\
$\beta_{2}$ & -1 & $-0,04274$ & $-0,02125$ & $-0,02968$ & $-0,02127$ & $-0,02832$ \\
$\sigma_{e}^{2}$ & 1 & 1,54260 & 1,55470 & 1,56840 & 1,56470 & 1,56860 \\
\hline
\end{tabular}

Tabela 4.18. Estimativas dos parâmetros segundo o modelo "escore corrigido"para diferentes tamanhos de amostra e Instrumento 3.

\begin{tabular}{|c|c|c|c|c|c|c|}
\hline Parâmetros & Valor & 500 & 1000 & 2000 & 3000 & 4000 \\
\hline$\beta_{0}$ & 1 & 1,01533 & 1,06063 & 1,02220 & 0,96927 & 0,99755 \\
$\beta_{1}$ & 4 & 4,19970 & 4,13940 & 3,96020 & 4,11650 & 4,11170 \\
$\beta_{2}$ & -1 & $-1,22810$ & $-1,00440$ & $-1,05923$ & $-1,09000$ & $-1,03510$ \\
$\sigma_{e}^{2}$ & 1 & 1,06247 & 1,18080 & 1,08340 & 1,13050 & 1,10680 \\
\hline
\end{tabular}

No instrumento 3, as estimativas obtidas pelo método "naive" não se aproximam aos valores fixados na presente simulação, enquanto os estimativas do método do "escore corrigido"se aproximam aos valores fixados. 


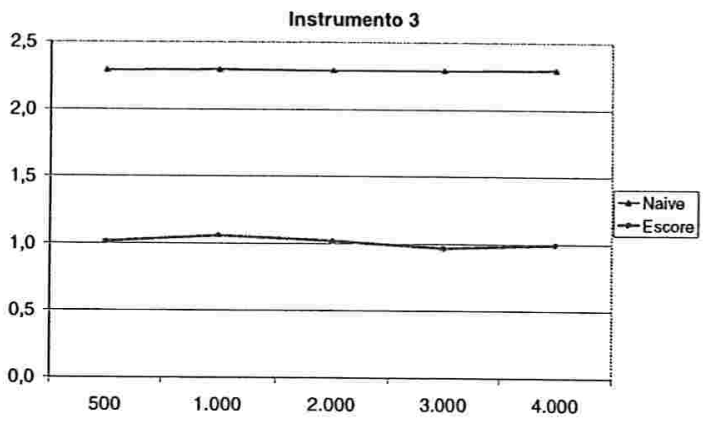

(a) Estimativa para $\beta_{0}$

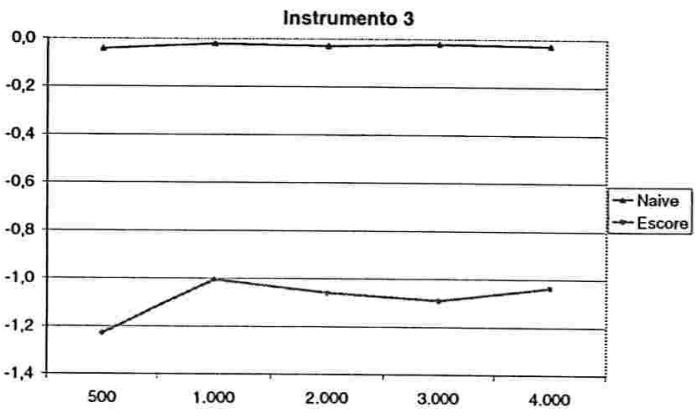

(c) Estimativa para $\beta_{2}$

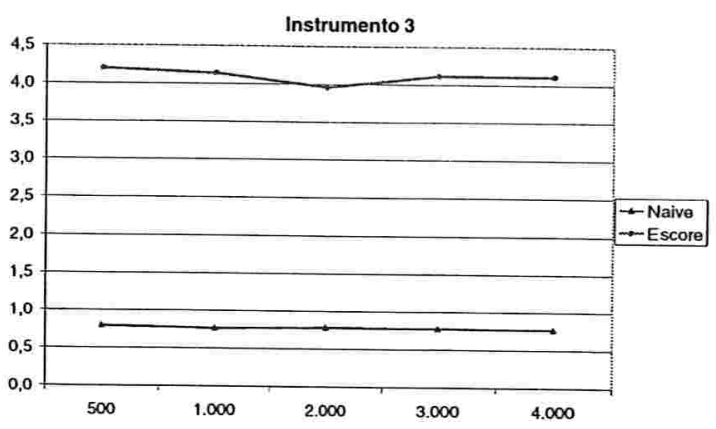

(b) Estimativa para $\beta_{1}$

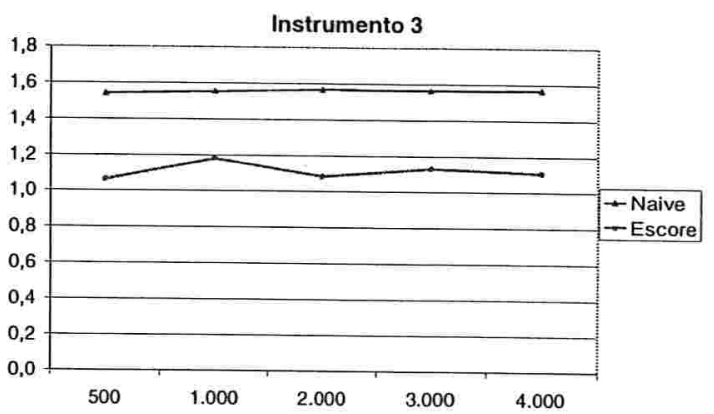

(d) Estimativa para $\beta_{2}$

Fig. 4.9. Comportamento das estimativas "naive" e "escore corrigido"segundo o tamanho da amostra no instrumento 3

Dos gráficos acima podemos observar que as estimativas do método "escore corrigido" se aproximam aos valores fixados, enquanto as estimativas do método "naive" não se aproximam a estes valores. 
4.5 Caso 2: $\lambda=\frac{\sigma_{e}^{2}}{\sigma_{u}^{2}}$ conhecida

a) Estimação dos parâmetros.

Nesta seção implementaremos a metodologia apresentada por Bolfarine e Gimenez (Escore Corrigida) em modelos polinomiais para calibração comparativa, para tal fim, estaremos utilizando os procedimentos vistos na seção 2.3 do capítulo 2 .

O modelo de calibração "naive"é como na equação (4.5), quando consideramos variâncias homogêneas a suposição para modelos de tipo funcional é

$$
\left(\begin{array}{l}
\mathbf{e}_{i} \\
u_{i}
\end{array}\right) \sim N_{r+1}\left[\left(\begin{array}{l}
0 \\
0
\end{array}\right),\left(\begin{array}{cc}
\sigma_{u}^{2} \Sigma_{\lambda \lambda} & 0 \\
0 & \sigma_{u}^{2}
\end{array}\right)\right],
$$

onde $\Sigma_{\lambda \lambda}=\left(\lambda_{1}, \lambda_{2}, \ldots, \lambda_{r}\right)$, enquanto para modelos de tipo estrutural a expressão (4.15) pode ser apresentado conjuntamente com a distribuição $X_{i} \sim N\left(\mu_{X}, \sigma_{X}^{2}\right), i=1,2, \ldots, n$. Consideramos que $\lambda$ é conhecida.

Para a estimação dos parâmetros consideramos um processo algorítmico de tipo Newton-Raphson, que segue a seguinte estrutura,

\section{Algoritmo para estimação dos parâmetros no caso de escore corrigido}

1. Considere um valor inicial para $\sigma_{u}^{2}$.

2. Substitua o valor considerado na etapa anterior na expressão seguinte,

$$
\left(\begin{array}{cccc}
n & \sum_{i=1}^{n} t_{i,(1)} & \cdots & \sum_{i=1}^{n} t_{i,(p)} \\
\sum_{i=1}^{n} t_{i,(1)} & \sum_{i=1}^{n} t_{i,(2)} & \cdots & \sum_{i=1}^{n} t_{i,(p+1)} \\
\vdots & \vdots & \cdots & \vdots \\
\sum_{i=1}^{n} t_{i,(p)} & \sum_{i=1}^{n} t_{i,(p+1)} & \cdots & \sum_{i=1}^{n} t_{i,(2 p)}
\end{array}\right)\left(\begin{array}{c}
\hat{\beta}_{0, k}^{(m)} \\
\hat{\beta}_{1, k}^{(m)} \\
\vdots \\
\hat{\beta}_{p, k}^{(m)}
\end{array}\right)=\left(\begin{array}{c}
\sum_{i=1}^{n} Y_{i, k} \\
\sum_{i=1}^{n} Y_{i, k} t_{i,(1)} \\
\vdots \\
\sum_{i=1}^{n} Y_{i, k} t_{i,(p)}
\end{array}\right)
$$

lembrando que cada valor de $t_{i,(s)}$ segue a forma da expressão (2.37). 
3. Agora com os valores $\beta_{j, k}^{(m)}, j=0,1, \ldots, p$ parâmetros $\beta$ e $k=1,2, \ldots, r$ instrumentos, obtidos devemos atualizar os valores de $\sigma_{u}^{2}$, para isto consideramos o método de Newton-Raphson em cada instrumento que segue segundo,

$$
\sigma_{u_{k}}^{2(m+1)}=\sigma_{u_{k}}^{2(m)}+\frac{U^{*}\left(\sigma_{u_{k}}^{2(m)}\right)}{I^{*}\left(\sigma_{u_{k}}^{2(m)}\right)},
$$

4. O novo valor de $\sigma_{u}^{2(m+1)}$ será a media obtida em cada instrumento, isto é,

$$
\sigma_{u}^{2(m+1)}=\frac{1}{r} \sum_{k=1}^{r} \sigma_{u_{k}}^{2(m+1)},
$$

\section{b) Aplicação numérica.}

Para esta seção consideramos uma simulação com as seguintes características:

1. São considerados três instrumentos a comparar em um modelo quadrático.

2. Se gera $X_{i} \sim \operatorname{Binomial}(10 ; 0,5), u_{i} \sim N(0 ; 0,25)$ e $\lambda_{1}=1, \lambda_{2}=2, \lambda_{3}=3$, sendo que $e_{i 1}, e_{i 2}, e_{i 3}$ são independentes.

3. Os parâmetros considerados na presente simulação são $\beta_{0}=(1,2,-1)^{t}, \beta_{1}=(2,-1,2)^{t}$ e $\beta_{2}=(-1,2,1)^{t}$.

4. Considerar os modelos seguintes

$$
\begin{aligned}
& W_{i}=X_{i}+u_{i} \\
& Y_{i 1}=\beta_{01}+\beta_{11} X_{i}+\beta_{21} X_{i}^{2}+e_{i 1} \\
& Y_{i 2}=\beta_{02}+\beta_{12} X_{i}+\beta_{22} X_{i}^{2}+e_{i 2} \\
& Y_{i 3}=\beta_{03}+\beta_{13} X_{i}+\beta_{23} X_{i}^{2}+e_{i 3}
\end{aligned}
$$

\section{Resultados}

Em seguida os resultados obtidos da simulação implementada acima em linguagem OX (Doornik, 1998) são apresentados nas tabelas seguintes 
Tabela 4.19. Estimativas dos parâmetros segundo o modelo "naive"para diferentes tamanhos de amostra e Instrumento 1.

\begin{tabular}{|c|c|c|c|c|c|c|}
\hline Parâmetros & Valores & 500 & 1000 & 2000 & 3000 & 4000 \\
$\beta_{0}$ & 1 & 1,54620 & 1,53970 & 1,54560 & 1,54620 & 1,54490 \\
$\beta_{1}$ & 2 & 0,27620 & 0,27551 & 0,27828 & 0,27524 & 0,27763 \\
$\beta_{2}$ & -1 & $-0,03074$ & $-0,02228$ & $-0,02770$ & $-0,02772$ & $-0,02904$ \\
$\sigma_{e}^{2}$ & 0,25 & 0,31345 & 0,31663 & 0,31807 & 0,31800 & 0,31775 \\
\hline
\end{tabular}

Tabela 4.20. Estimativas dos parâmetros segundo o modelo "escore corrigido"para diferentes tamanhos de amostra e Instrumento 1.

\begin{tabular}{|c|c|c|c|c|c|c|}
\hline Parâmetros & Valores & 500 & 1000 & 2000 & 3000 & 4000 \\
$\beta_{0}$ & 1 & 1,03100 & 0,92095 & 1,00470 & 1,01380 & 0,97888 \\
$\beta_{1}$ & 2 & 0,97947 & 2,39530 & 1,99410 & 1,94100 & 2,11450 \\
$\beta_{2}$ & -1 & $-1,02830$ & $-1,06300$ & $-0,99747$ & $-0,95048$ & $-1,10990$ \\
$\sigma_{e}^{2}$ & 0,25 & 0,23105 & 0,23227 & 0,22633 & 0,22112 & 0,27462 \\
\hline
\end{tabular}

Das tabelas acima podemos apreciar que as estimativas do método de "escore corrigido" se aproxima adequadamente aos valores fixados na simulação para o instrumento 1. 


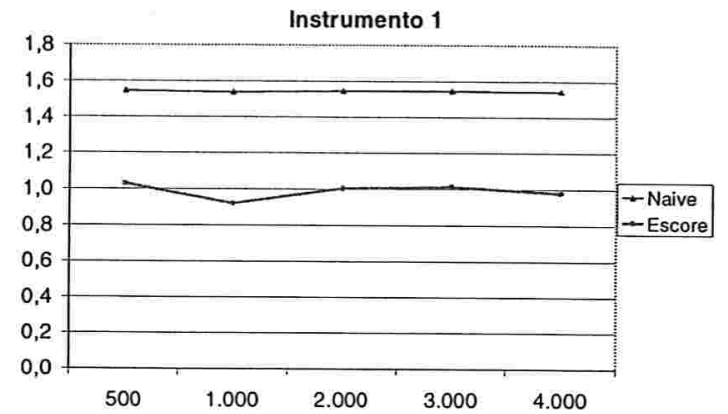

(a) Estimativa para $\beta_{0}$

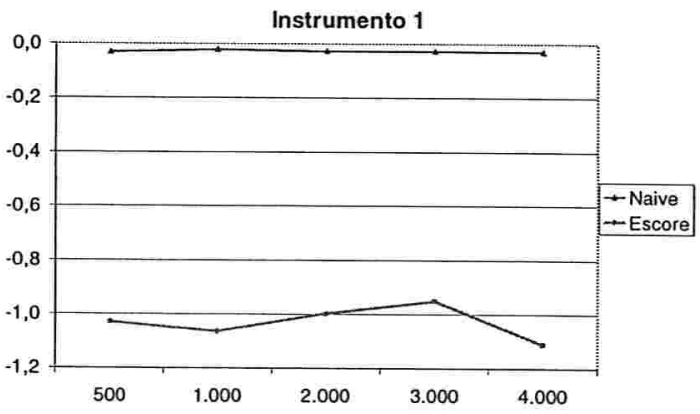

(c) Estimativa para $\beta_{2}$

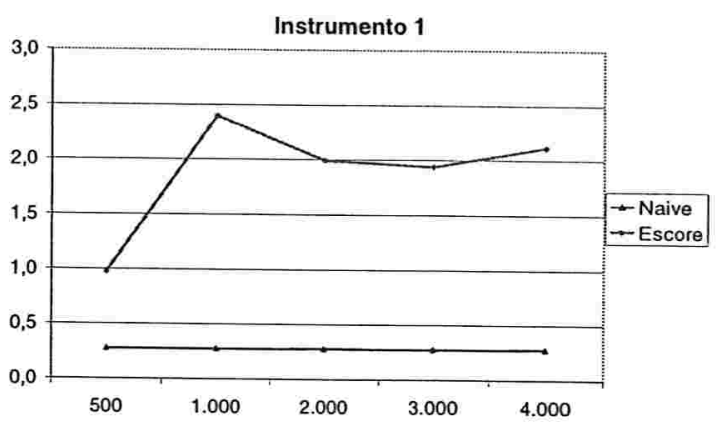

(b) Estimativa para $\beta_{1}$

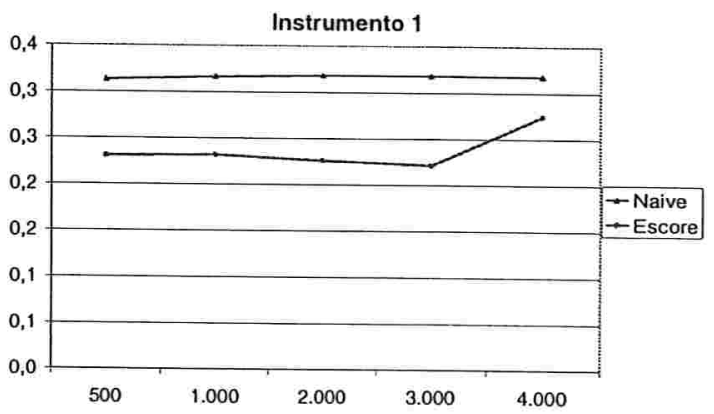

(d) Estimativa para $\beta_{2}$

Fig. 4.10. Comportamento das estimativas "naive"e "escore corrigido"segundo o tamanho da amostra no instrumento 1

Segundo os gráficos acima amostram o bom ajuste das estimativas do método do "escore corrigido" a os valores fixados. Também, observa-se que as estimativas do método "naive" não conseguem aproximar-se a os valores fixados para o instrumento 1. 
Tabela 4.21. Estimativas dos parâmetros segundo o modelo "naive" para diferentes tamanhos de amostra e Instrumento 2 .

\begin{tabular}{|c|c|c|c|c|c|c|}
\hline Parâmetros & Valores & 500 & 1000 & 2000 & 3000 & 4000 \\
$\beta_{0}$ & 2 & 2,03750 & 2,03630 & 2,03480 & 2,03790 & 2,03890 \\
$\beta_{1}$ & -1 & 0,19495 & 0,19720 & 0,19944 & 0,19724 & 0,19359 \\
$\beta_{2}$ & 2 & 0,05216 & 0,05414 & 0,04889 & 0,05162 & 0,05480 \\
$\sigma_{e}^{2}$ & 0,5 & 0,57638 & 0,58671 & 0,58325 & 0,58174 & 0,58613 \\
\hline
\end{tabular}

Tabela 4.22. Estimativas dos parâmetros segundo o modelo "escore corrigido" para diferentes tamanhos de amostra e Instrumento 2.

\begin{tabular}{|c|c|c|c|c|c|c|}
\hline Parâmetros & Valores & 500 & 1000 & 2000 & 3000 & 4000 \\
$\beta_{0}$ & 2 & 2,02340 & 2,04850 & 1,98750 & 2,02660 & 2,00190 \\
$\beta_{1}$ & -1 & $-0,97271$ & $-1,07570$ & $-0,95198$ & $-1,16690$ & $-0,98823$ \\
$\beta_{2}$ & 2 & 2,00450 & 2,28050 & 1,95130 & 2,17160 & 1,98050 \\
$\sigma_{e}^{2}$ & 0,5 & 0,46210 & 0,66454 & 0,55265 & 0,54223 & 0,54923 \\
\hline
\end{tabular}

Para o instrumento 2 pode-se observar que as estimativas do método do "escore corrigido" tem uma boa proximidade aos valores fixados na simulação que as estimativas do método "naive", gráficamente este comportamento se expressa nas figuras seguintes, 


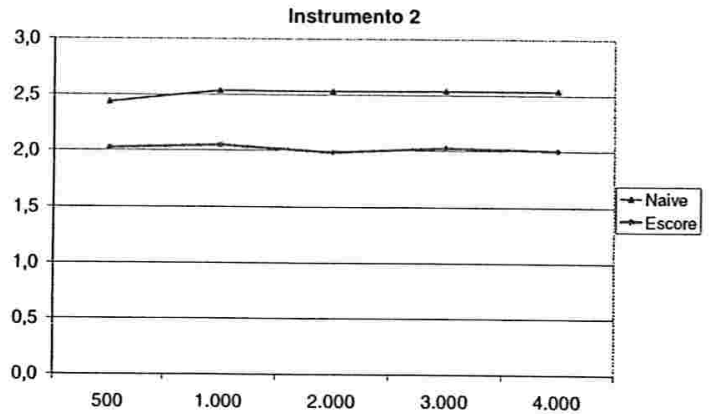

(a) Estimativa para $\beta_{0}$

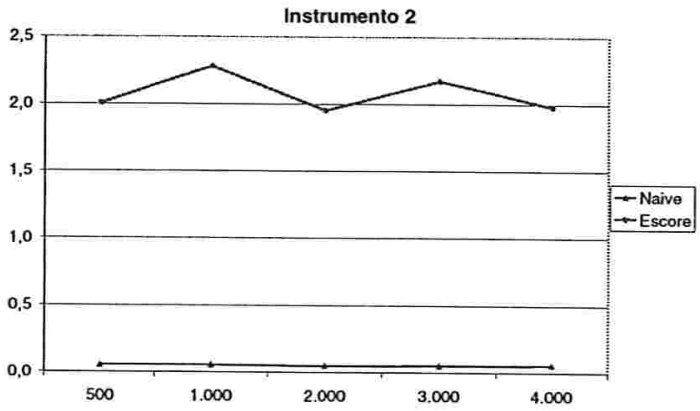

(c) Estimativa para $\beta_{2}$

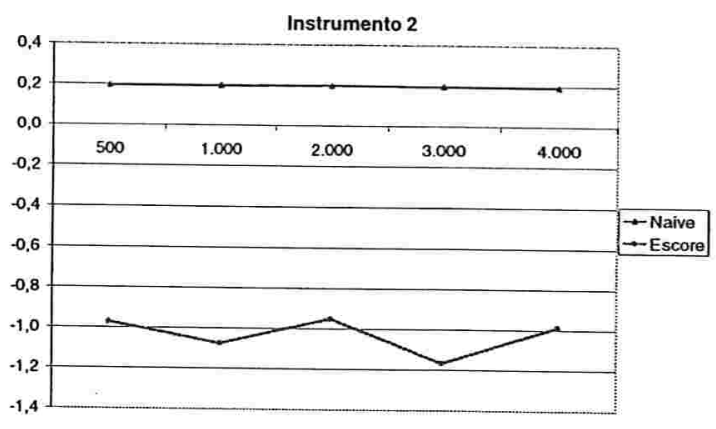

(b) Estimativa para $\beta_{1}$

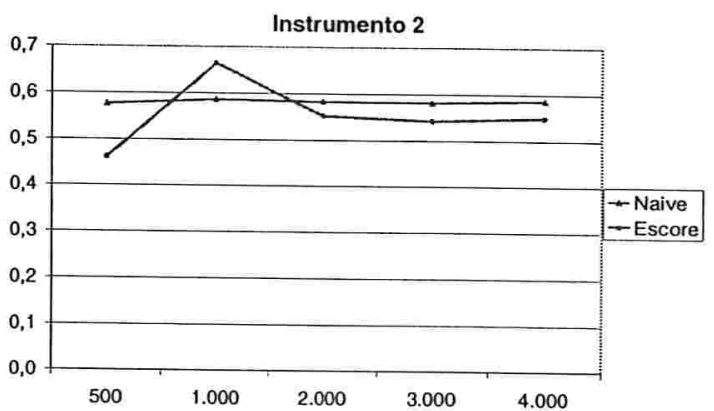

(d) Estimativa para $\beta_{2}$

Fig. 4.11. Comportamento das estimativas "naive"e "escore corrigido"segundo o tamanho da amostra no instrumento 2

Destas figuras pode-se observar que as estimativas do método "naive" não se aproximam aos valores fixados, enquanto as estimativas do método "escore corrigido" se aproximam razoávelmente aos valores fixados no instrumento 2 . 
Tabela 4.23. Estimativas dos parâmetros segundo o modelo "naive" para diferentes tamanhos de amostra e Instrumento 3.

\begin{tabular}{|c|c|c|c|c|c|c|}
\hline Parâmetros & Valores & 500 & 1000 & 2000 & 3000 & 4000 \\
$\beta_{0}$ & -1 & $-0,03839$ & $-0,04120$ & $-0,04536$ & $-0,04080$ & $-0,04039$ \\
$\beta_{1}$ & 2 & 0,72362 & 0,73019 & 0,72963 & 0,71675 & 0,72654 \\
$\beta_{2}$ & 1 & 0,01500 & 0,02375 & 0,01988 & 0,03051 & 0,02186 \\
$\sigma_{e}^{2}$ & 0,75 & 1,31180 & 1,30900 & 1,31240 & 1,32150 & 1,31550 \\
\hline
\end{tabular}

Tabela 4.24. Estimativas dos parâmetros segundo o modelo "escore corrigido"para diferentes tamanhos de amostra e Instrumento 3.

\begin{tabular}{|c|c|c|c|c|c|c|}
\hline Parâmetros & Valores & 500 & 1000 & 2000 & 3000 & 4000 \\
$\beta_{0}$ & -1 & $-1,11663$ & $-1,05790$ & $-1,02320$ & $-0,94406$ & $-1,03250$ \\
$\beta_{1}$ & 2 & 2,23079 & 2,13090 & 2,16800 & 1,95600 & 2,01120 \\
$\beta_{2}$ & 1 & 1,00149 & 0,90639 & 0,90986 & 1,15480 & 0,98565 \\
$\sigma_{e}^{2}$ & 0,75 & 0,69320 & 0,79681 & 0,77898 & 0,76335 & 0,74385 \\
\hline
\end{tabular}

Da mesma maneira no instrumento 3, as estimativas do método "naive" não se aproximam aos valores fixados na presente simulação, enquanto as estimativas do método do "escore corrigido" tem uma boa aproximação aos valores fixados na simulação. 


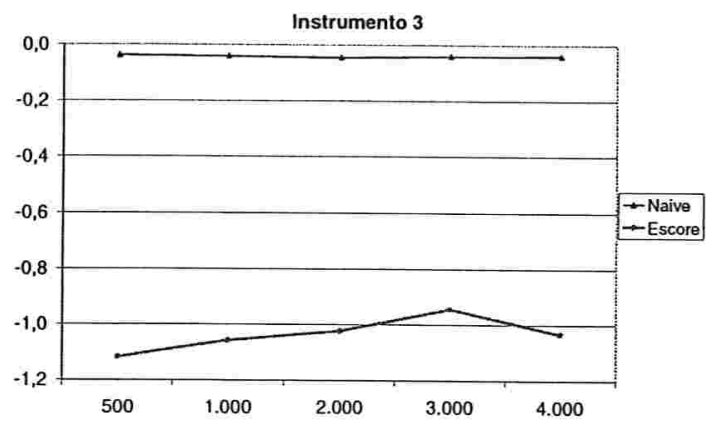

(a) Estimativa para $\beta_{0}$

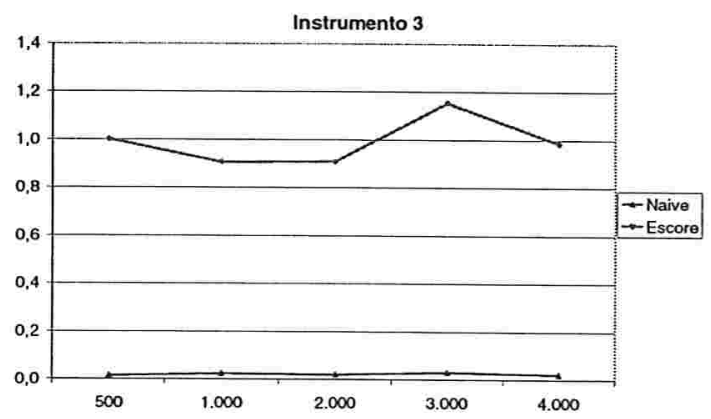

(c) Estimativa para $\beta_{2}$

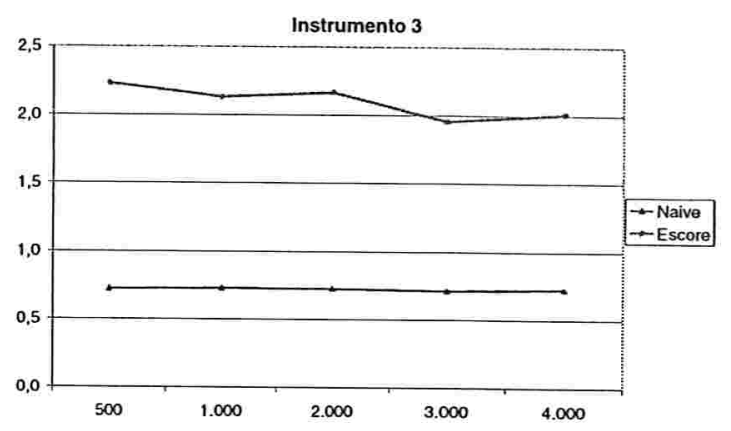

(b) Estimativa para $\beta_{1}$

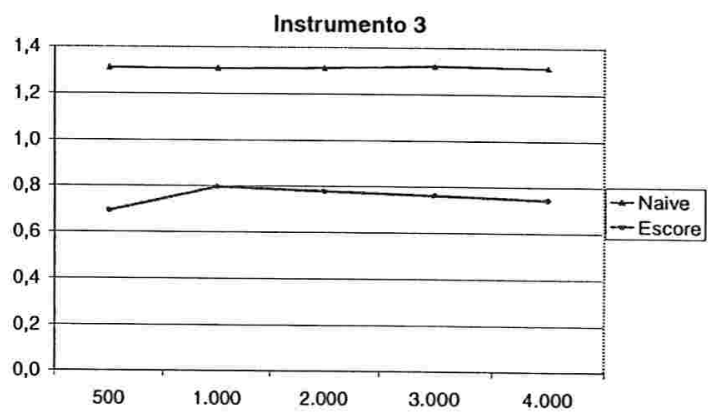

(d) Estimativa para $\beta_{2}$

Fig. 4.12. Comportamento das estimativas "naive"e "escore corrigido"segundo o tamanho da amostra no instrumento 3

Dos gráficos acima, pode observar-se que no caso das estimativas do método do "escore corrigido" estas apresentam boa aproximação aos valores fixados na simulação, enquanto as estimativas do método "naive" não conseguem ter uma aproximação adequada aos valores propostos. 


\section{CAPÍTULO}

\section{$\begin{array}{r}O \\ \hline\end{array}$ CONSIDERAÇÕES FINAIS}

O objetivo principal do presente trabalho foi a de avaliar a metodologia proposta por Nakamura (1990) em modelos polinomiais com erros nas variáveis, no decorrer do presente trabalhos obtivemos que a metodologia proposta por Nakamura (1990) pode-se aplicar adequadamente aos modelos polinomiais com erros nas variáveis quando $\sigma_{u}^{2}$ é conhecida, isto é assim, devido a que a função verossimilhança corrigida produz a função de escore corrigida pela derivada simple do parâmetro.

Esta metodologia em modelos aditivos obtém estimativas próximas aos valores fixados com tamanhos de amostra menores que os modelos multiplicativos.

Quando $\sigma_{e}^{2}$ não é conhecida diretamente a metodologia proposta por Nakamura (1990) não é conveniente, devido a que a função verossimilhança corrigida não produz a função escore corrigido de $\sigma_{u}^{2}$, este fato é devido à falta do suposto de permutabilidade da derivada e o a esperança da variável que Nakamura manifesta em seu trabalho.

Frente a este problema se considerou trabalhar com a metodologia proposta por Bolfarine e Gimenez (1997), esta metodologia propõe trabalhar a correção da função escore e não a da função verossimilhança, permitindo obter boas estimativas.

Quando foi comparado o tamanho de amostra necessário para a simulação observouse que também na metodologia proposta por Bolfarine e Gimenez (1997) foi preciso aumentar o tamanho de amostra para os modelos multiplicativos, já que com tamanhos de amostra 
proposto em modelos aditivos não se conseguia obter estimativas próximas aos valores fixados na simulação.

As metodologias estudadas no presente trabalho poderiam ser estendidas a modelos mais complexos que muitas vezes são considerados na econometria, por exemplo, poderia ser considerada o modelo

$$
Y_{i}=\beta_{0}+\beta_{1} X_{i 1}+\beta_{2} X_{i 1}^{2}+\beta_{3} X_{i 2}
$$

sendo

$$
W_{i 1}=X_{i 1}+u_{i 1}, \quad \text { ou } \quad W_{i 1}=X_{i 1} u_{i 1}
$$

e

$$
W_{i 2}=X_{i 2}+u_{i 2}, \quad \text { ou } \quad W_{i 2}=X_{i 2} u_{i 2}
$$

Uma outra extensão que poderia ser considerada é estudar os modelos ultraestrutural que não foi considerada no presente trabalho.

Este trabalho poderia ser estendido aos modelos polinomiais de Berkson considerando as metodologias propostas por Nakamura (1990) e Bolfarine e Gimenez (1997). 


\section{CAPÍTULO}

Anexo

\section{Anexo}

Tabela 6.1. Dados simulados por Fuller (1987) para um polinômio de segundo grau.

\begin{tabular}{|c|c|c|c|c|c|c|c|c|c|c|c|}
\hline No & $\mathrm{Y}_{1}$ & $\mathrm{Y}_{2}$ & $\mathrm{~W}$ & $\mathrm{No}_{\mathrm{O}}$ & $\mathrm{Y}_{1}$ & $\mathrm{Y}_{2}$ & $\mathrm{~W}$ & $\mathrm{No}$ & $\mathrm{Y}_{1}$ & $\mathrm{Y}_{2}$ & $\mathrm{~W}$ \\
\hline 1 & 0,7 & 1,24 & $-1,44$ & 17 & 0,1 & 0,64 & $-1,11$ & 33 & 0,2 & 0,47 & $-0,22$ \\
2 & 0,05 & 0,59 & $-0,9$ & 18 & 0,31 & 0,85 & $-0,57$ & 34 & 0,16 & 0,43 & $-0,98$ \\
3 & 0,99 & 1,53 & $-0,81$ & 19 & 0,24 & 0,78 & $-0,9$ & 35 & 0,45 & 0,72 & $-0,54$ \\
4 & 0,3 & 0,84 & $-1,14$ & 20 & 0,13 & 0,67 & $-1,25$ & 36 & 0,42 & 0,69 & $-0,54$ \\
5 & 1,1 & 1,64 & $-1,36$ & 21 & 0,45 & 0,99 & $-0,81$ & 37 & 0,14 & 0,41 & $-0,81$ \\
6 & 0,93 & 1,47 & $-0,61$ & 22 & 0,66 & 1,19 & $-1,07$ & 38 & $-0,21$ & 0,06 & $-0,14$ \\
7 & 0,3 & 0,84 & $-1,11$ & 23 & 0,18 & 0,72 & $-0,51$ & 39 & $-0,59$ & $-0,31$ & $-1,03$ \\
8 & 0,24 & 0,78 & $-1,46$ & 24 & 0,47 & 1,01 & $-0,86$ & 40 & $-0,22$ & 0,05 & $-0,36$ \\
9 & 0,28 & 0,82 & $-0,77$ & 25 & 0,18 & 0,45 & $-0,16$ & 41 & $-0,25$ & 0,02 & $-0,14$ \\
10 & 0,86 & 1,4 & $-1,31$ & 26 & $-0,18$ & 0,09 & $-0,64$ & 42 & $-0,23$ & 0,04 & $-0,37$ \\
11 & 0,76 & 1,3 & $-0,98$ & 27 & 0,15 & 0,42 & 0,21 & 43 & $-0,13$ & 0,14 & $-0,71$ \\
12 & 0,48 & 1,02 & $-0,93$ & 28 & 0,12 & 0,39 & $-0,5$ & 44 & $-0,41$ & $-0,14$ & $-0,21$ \\
13 & 0,11 & 0,65 & $-1,02$ & 29 & 0,05 & 0,32 & $-0,45$ & 45 & 0,02 & 0,29 & $-0,52$ \\
14 & 0,21 & 0,75 & $-1,6$ & 30 & $-0,28$ & $-0,01$ & $-0,71$ & 46 & 0,34 & 0,61 & $-1,01$ \\
15 & 0,6 & 1,14 & $-0,45$ & 31 & $-0,59$ & $-0,32$ & $-0,7$ & 47 & $-0,31$ & $-0,04$ & $-0,34$ \\
16 & 0,58 & 1,12 & $-1,07$ & 32 & 0,38 & 0,65 & $-0,46$ & 48 & 0,31 & 0,58 & $-0,68$ \\
\hline
\end{tabular}


Tabela 6.2. Dados simulados por Fuller (1987) para um polinômio de segundo grau (Continuação...).

\begin{tabular}{|c|c|c|c|c|c|c|c|c|c|c|c|}
\hline Obs & $\mathrm{Y}_{1}$ & $\mathrm{Y}_{2}$ & $\mathrm{~W}$ & $\mathrm{Obs}$ & $\mathrm{Y}_{1}$ & $\mathrm{Y}_{2}$ & $\mathrm{~W}$ & $\mathrm{Obs}$ & $\mathrm{Y}_{1}$ & $\mathrm{Y}_{2}$ & $\mathrm{~W}$ \\
\hline 49 & $-0,06$ & $-0,06$ & $-0,40$ & 73 & 0,05 & $-0,22$ & 0,83 & 97 & 1,54 & 1,00 & 1,03 \\
50 & 0,35 & $-0,35$ & $-0,06$ & 74 & 0,51 & 0,24 & 0,70 & 98 & 1,53 & 0,99 & 0,92 \\
51 & $-0,35$ & $-0,35$ & 0,13 & 75 & 0,69 & 0,42 & 0,35 & 99 & 1,32 & 0,78 & 1,28 \\
52 & 0,09 & 0,09 & $-0,18$ & 76 & 0,20 & $-0,07$ & 0,64 & 100 & 1,48 & 0,94 & 1,53 \\
53 & 0,11 & 0,11 & 0,11 & 77 & 1,08 & 0,81 & 0,52 & 101 & 1,03 & 0,49 & 1,22 \\
54 & 0,03 & 0,03 & $-0,04$ & 78 & 0,70 & 0,43 & 0,67 & 102 & 1,11 & 0,57 & 0,84 \\
55 & 0,26 & 0,26 & $-0,54$ & 79 & 0,17 & $-0,10$ & 0,50 & 103 & 1,33 & 0,79 & 1,23 \\
56 & 0,41 & 0,41 & $-0,10$ & 80 & 0,39 & 0,12 & $-0,26$ & 104 & 1,43 & 0,89 & 1,36 \\
57 & $-0,01$ & $-0,01$ & 0,35 & 81 & 0,68 & 0,41 & 0,56 & 105 & 1,76 & 1,22 & 0,72 \\
58 & $-0,45$ & $-0,45$ & $-0,32$ & 82 & 0,87 & 0,60 & 0,44 & 106 & 1,16 & 0,62 & 0,71 \\
59 & 0,32 & 0,32 & $-0,23$ & 83 & 0,79 & 0,52 & 0,99 & 107 & 1,96 & 1,42 & 1,44 \\
60 & $-0,14$ & $-0,14$ & $-0,12$ & 84 & 0,05 & $-0,22$ & 0,76 & 108 & 2,05 & 1,51 & 0,82 \\
61 & $-0,06$ & $-0,06$ & $-0,05$ & 85 & 0,43 & 0,16 & 0,55 & 109 & 1,80 & 1,26 & 0,58 \\
62 & 0,32 & 0,32 & 0,31 & 86 & 0,17 & $-0,10$ & 0,53 & 110 & 1,63 & 1,09 & 0,39 \\
63 & $-0,06$ & $-0,06$ & $-0,45$ & 87 & 0,40 & 0,13 & 0,89 & 111 & 0,92 & 0,38 & 0,63 \\
64 & $-0,22$ & $-0,22$ & 0,65 & 88 & 0,33 & 0,06 & 0,35 & 112 & 1,86 & 1,32 & 1,11 \\
65 & $-0,30$ & $-0,30$ & 0,57 & 89 & 0,73 & 0,46 & 0,33 & 113 & 1,50 & 0,96 & 0,84 \\
66 & $-0,26$ & $-0,26$ & 0,17 & 90 & 0,57 & 0,30 & 0,39 & 114 & 2,04 & 1,50 & 1,07 \\
67 & $-0,59$ & $-0,59$ & $-0,16$ & 91 & 0,57 & 0,30 & $-0,19$ & 115 & 1,85 & 1,31 & 0,80 \\
68 & $-0,31$ & $-0,31$ & $-0,02$ & 92 & 0,36 & 0,09 & 0,55 & 116 & 1,34 & 0,80 & 1,08 \\
69 & 0,23 & 0,23 & 0,08 & 93 & 0,57 & 0,30 & 0,55 & 117 & 1,60 & 1,06 & 0,85 \\
70 & $-0,25$ & $-0,25$ & $-0,17$ & 94 & 0,64 & 0,37 & 0,02 & 118 & 1,56 & 1,02 & 0,97 \\
71 & 0,59 & 0,59 & 0,09 & 95 & 0,27 & 0,00 & 0,46 & 119 & 1,60 & 1,06 & 1,00 \\
72 & 0,34 & 0,34 & 0,43 & 96 & 1,25 & 0,98 & 0,84 & 120 & 1,57 & 1,03 & 1,60 \\
\hline
\end{tabular}


Tabela 6.3. Dados do exemplo 4 em Galea-Rojas et. al. (2003).

\begin{tabular}{|c|c|c|c|c|c|c|c|}
\hline $\mathrm{W}$ & $\sigma_{u}^{2}$ & $\mathrm{Y}$ & $\sigma_{e}^{2}$ & $\mathrm{~W}$ & $\sigma_{u}^{2}$ & $\mathrm{Y}$ & $\sigma_{e}^{2}$ \\
\hline 0,229 & 0,074693 & 0,330 & 0,042531 & 0,134 & 0,057042 & 0,180 & 0,031355 \\
0,487 & 0,109180 & 0,601 & 0,057543 & 0,347 & 0,092063 & 0,325 & 0,042223 \\
0,257 & 0,079156 & 0,292 & 0,040004 & 1,032 & 0,159305 & 1,442 & 0,089488 \\
0,894 & 0,148205 & 0,294 & 0,040114 & 0,138 & 0,057892 & 0,278 & 0,038998 \\
0,131 & 0,056396 & 0,398 & 0,046731 & 1,256 & 0,175852 & 1,206 & 0,081788 \\
0,122 & 0,054412 & 0,241 & 0,036256 & 1,164 & 0,169249 & 1,837 & 0,101136 \\
0,092 & 0,047209 & 0,110 & 0,024383 & 0,067 & 0,040248 & 0,467 & 0,050647 \\
0,540 & 0,115004 & 0,663 & 0,060450 & 0,081 & 0,044280 & 0,381 & 0,045720 \\
0,211 & 0,071679 & 0,319 & 0,041805 & 0,136 & 0,057468 & 0,079 & 0,020667 \\
0,164 & 0,063144 & 0,182 & 0,031478 & 0,250 & 0,078064 & 0,563 & 0,055657 \\
0,149 & 0,060169 & 0,149 & 0,028464 & 0,040 & 0,031049 & 0,074 & 0,019992 \\
0,116 & 0,053049 & 0,117 & 0,025207 & 0,040 & 0,031049 & 0,242 & 0,036347 \\
0,246 & 0,077433 & 0,273 & 0,038657 & 0,538 & 0,114790 & 0,439 & 0,049123 \\
0,274 & 0,081748 & 0,300 & 0,040548 & 0,368 & 0,094825 & 0,214 & 0,034176 \\
0,347 & 0,092063 & 0,384 & 0,045902 & 0,144 & 0,059145 & 0,108 & 0,024213 \\
0,381 & 0,096496 & 0,149 & 0,028506 & 0,062 & 0,038708 & 0,054 & 0,017132 \\
0,241 & 0,076637 & 0,285 & 0,039509 & 0,140 & 0,058313 & 0,343 & 0,043378 \\
0,200 & 0,069774 & 0,282 & 0,039241 & 0,070 & 0,041145 & 0,168 & 0,030260 \\
0,115 & 0,052818 & 0,156 & 0,029126 & 0,313 & 0,087409 & 0,612 & 0,058053 \\
0,150 & 0,060372 & 0,615 & 0,058194 & 0,693 & 0,130382 & 0,526 & 0,053794 \\
0,040 & 0,031049 & 0,059 & 0,017809 & 0,096 & 0,048231 & 0,113 & 0,024713 \\
0,561 & 0,117233 & 0,462 & 0,050414 & 0,125 & 0,055081 & 0,160 & 0,029529 \\
0,151 & 0,060574 & 0,143 & 0,027900 & 0,175 & 0,065241 & 0,346 & 0,043560 \\
0,141 & 0,058522 & 0,146 & 0,028213 & 0,213 & 0,072020 & 0,164 & 0,029850 \\
0,136 & 0,057468 & 0,104 & 0,023781 & 0,495 & 0,110078 & 0,497 & 0,052292 \\
\hline
\end{tabular}


Tabela 6.4. Dados do exemplo 4 em Galea-Rojas et. al. (2003).

(Continuação...).

\begin{tabular}{|c|c|c|c|c|c|c|c|}
\hline $\mathrm{W}$ & $\sigma_{u}^{2}$ & $\mathrm{Y}$ & $\sigma_{e}^{2}$ & $\mathrm{~W}$ & $\sigma_{u}^{2}$ & $\mathrm{Y}$ & $\sigma_{e}^{2}$ \\
\hline 0,074 & 0,042311 & 0,142 & 0,027738 & 0,205 & 0,070646 & 0,153 & 0,028842 \\
0,040 & 0,031049 & 0,040 & 0,014657 & 0,093 & 0,047467 & 0,068 & 0,019118 \\
0,196 & 0,069069 & 0,186 & 0,031835 & 0,088 & 0,046165 & 0,165 & 0,029929 \\
0,513 & 0,112074 & 0,454 & 0,049972 & 0,081 & 0,044280 & 0,071 & 0,019581 \\
0,080 & 0,044004 & 0,088 & 0,021777 & 0,111 & 0,051886 & 0,130 & 0,026572 \\
0,241 & 0,076637 & 0,133 & 0,026832 & 0,040 & 0,031049 & 0,039 & 0,014461 \\
0,082 & 0,044554 & 0,072 & 0,019761 & 0,260 & 0,079619 & 0,204 & 0,033345 \\
0,331 & 0,089902 & 0,472 & 0,050952 & 0,040 & 0,031049 & 0,050 & 0,016381 \\
0,279 & 0,082495 & 0,259 & 0,037642 & 0,176 & 0,065428 & 0,177 & 0,031043 \\
0,743 & 0,135033 & 0,516 & 0,053303 & 0,152 & 0,060776 & 0,155 & 0,029040 \\
0,111 & 0,051886 & 0,102 & 0,023496 & 0,040 & 0,031049 & 0,131 & 0,026661 \\
0,088 & 0,046165 & 0,128 & 0,026337 & 0,122 & 0,054412 & 0,186 & 0,031867 \\
0,585 & 0,119730 & 0,652 & 0,059941 & 0,077 & 0,043166 & 0,068 & 0,019088 \\
1,146 & 0,167928 & 0,664 & 0,060507 & 1,011 & 0,157665 & 0,207 & 0,033602 \\
0,204 & 0,070473 & 0,254 & 0,037225 & 0,806 & 0,140677 & 0,422 & 0,048146 \\
0,108 & 0,051175 & 0,097 & 0,022861 & 0,189 & 0,067816 & 0,134 & 0,026983 \\
0,137 & 0,057681 & 0,111 & 0,024518 & 0,161 & 0,062560 & 0,147 & 0,028299 \\
0,128 & 0,055742 & 0,119 & 0,025395 & 0,360 & 0,093783 & 0,216 & 0,034307 \\
0,205 & 0,070646 & 0,197 & 0,032740 & 1,062 & 0,161618 & 1,041 & 0,075917 \\
0,067 & 0,040248 & 0,056 & 0,017437 & 0,085 & 0,045367 & 0,093 & 0,022459 \\
0,321 & 0,088526 & 0,923 & 0,071444 & 0,287 & 0,083677 & 0,213 & 0,034119 \\
1,381 & 0,184450 & 1,023 & 0,075241 & 0,040 & 0,031049 & 0,120 & 0,025549 \\
0,096 & 0,048231 & 0,084 & 0,021310 & 0,071 & 0,041439 & 0,062 & 0,018238 \\
0,141 & 0,058522 & 0,190 & 0,032214 & 0,225 & 0,074034 & 0,175 & 0,030896 \\
0,336 & 0,090583 & 0,181 & 0,031362 & 0,202 & 0,070124 & 0,189 & 0,032113 \\
\hline
\end{tabular}


Tabela 6.5. Dados do exemplo 4 em Galea-Rojas et. al. (2003).

(Continuação...).

\begin{tabular}{|c|c|c|c|c|c|c|c|}
\hline $\mathrm{W}$ & $\sigma_{u}^{2}$ & $\mathrm{Y}$ & $\sigma_{e}^{2}$ & $\mathrm{~W}$ & $\sigma_{u}^{2}$ & $\mathrm{Y}$ & $\sigma_{e}^{2}$ \\
\hline 0,060 & 0,038074 & 0,061 & 0,018088 & 0,040 & 0,031049 & 0,060 & 0,017968 \\
0,757 & 0,136307 & 1,135 & 0,079317 & 0,298 & 0,085275 & 0,359 & 0,044381 \\
0,616 & 0,122881 & 0,751 & 0,064376 & 0,122 & 0,054412 & 0,149 & 0,028493 \\
0,088 & 0,046165 & 0,099 & 0,023181 & 0,177 & 0,065615 & 0,209 & 0,033761 \\
0,091 & 0,046950 & 0,116 & 0,025075 & 0,189 & 0,067816 & 0,188 & 0,031986 \\
0,098 & 0,048734 & 0,090 & 0,022056 & 0,544 & 0,115432 & 0,295 & 0,040151 \\
0,061 & 0,038392 & 0,105 & 0,023887 & 0,252 & 0,078377 & 0,508 & 0,052875 \\
0,264 & 0,080233 & 0,289 & 0,039775 & 0,094 & 0,047723 & 0,097 & 0,022911 \\
0,154 & 0,061177 & 0,148 & 0,028364 & 0,098 & 0,048734 & 0,108 & 0,024213 \\
0,348 & 0,092197 & 0,709 & 0,062550 & 0,094 & 0,047723 & 0,126 & 0,026103 \\
0,115 & 0,052818 & 0,154 & 0,028920 & 0,839 & 0,143546 & 0,562 & 0,055606 \\
0,622 & 0,123482 & 0,746 & 0,064153 & 0,399 & 0,098763 & 0,388 & 0,046148 \\
0,385 & 0,097004 & 0,617 & 0,058302 & 0,159 & 0,062168 & 0,191 & 0,032267 \\
0,229 & 0,074693 & 0,143 & 0,027854 & 0,475 & 0,107818 & 0,408 & 0,047332 \\
0,138 & 0,057892 & 0,137 & 0,027260 & 0,100 & 0,049232 & 0,109 & 0,024317 \\
0,164 & 0,063144 & 0,168 & 0,030273 & 0,103 & 0,049969 & 0,120 & 0,025514 \\
0,850 & 0,144490 & 0,566 & 0,055836 & 0,117 & 0,053278 & 0,204 & 0,033312 \\
0,206 & 0,070819 & 0,208 & 0,033715 & 0,079 & 0,043726 & 0,187 & 0,031939 \\
0,063 & 0,039021 & 0,075 & 0,020064 & 0,343 & 0,091528 & 0,536 & 0,054296 \\
0,103 & 0,049969 & 0,089 & 0,021903 & 0,124 & 0,054859 & 0,340 & 0,043172 \\
0,211 & 0,071679 & 0,210 & 0,033811 & 0,335 & 0,090447 & 0,490 & 0,051907 \\
0,040 & 0,031049 & 0,038 & 0,014376 & 0,090 & 0,046690 & 0,100 & 0,023281 \\
0,160 & 0,062365 & 0,219 & 0,034571 & 0,272 & 0,081447 & 0,325 & 0,042183 \\
0,080 & 0,044004 & 0,041 & 0,014891 & 0,321 & 0,088526 & 0,351 & 0,043855 \\
0,061 & 0,038392 & 0,087 & 0,021704 & 0,105 & 0,050455 & 0,124 & 0,025900 \\
\hline
\end{tabular}


Tabela 6.6. Dados do exemplo 4 em Galea-Rojas et. al. (2003).

(Continuação...).

\begin{tabular}{|c|c|c|c|c|c|c|c|}
\hline $\mathrm{W}$ & $\sigma_{u}^{2}$ & $\mathrm{Y}$ & $\sigma_{e}^{2}$ & $\mathrm{~W}$ & $\sigma_{u}^{2}$ & $\mathrm{Y}$ & $\sigma_{e}^{2}$ \\
\hline 0,477 & 0,108046 & 0,444 & 0,049408 & 0,222 & 0,073535 & 0,267 & 0,038190 \\
0,103 & 0,049969 & 0,304 & 0,040818 & 0,040 & 0,031049 & 0,040 & 0,014580 \\
0,123 & 0,054636 & 0,127 & 0,026295 & 0,141 & 0,058522 & 0,185 & 0,031737 \\
0,040 & 0,031049 & 0,063 & 0,018482 & 0,252 & 0,078377 & 0,329 & 0,042439 \\
0,132 & 0,056612 & 0,094 & 0,022592 & 0,308 & 0,086703 & 0,262 & 0,037842 \\
0,040 & 0,031049 & 0,074 & 0,019955 & 0,134 & 0,057042 & 0,151 & 0,028610 \\
0,161 & 0,062560 & 0,209 & 0,033788 & 0,100 & 0,049232 & 0,123 & 0,025882 \\
0,164 & 0,063144 & 0,249 & 0,036905 & 0,231 & 0,075020 & 0,334 & 0,042784 \\
0,225 & 0,074034 & 0,200 & 0,033027 & 0,740 & 0,134759 & 1,117 & 0,078672 \\
1,083 & 0,163218 & 1,022 & 0,075210 & 0,099 & 0,048984 & 0,099 & 0,023193 \\
0,061 & 0,038392 & 0,095 & 0,022666 & 0,189 & 0,067816 & 0,191 & 0,032226 \\
0,146 & 0,059557 & 0,152 & 0,028752 & 0,099 & 0,048984 & 0,149 & 0,028502 \\
0,097 & 0,048483 & 0,152 & 0,028785 & 0,040 & 0,031049 & 0,051 & 0,016619 \\
0,558 & 0,116917 & 0,949 & 0,072452 & 0,040 & 0,031049 & 0,040 & 0,014657 \\
0,451 & 0,105042 & 0,566 & 0,055809 & 0,267 & 0,080691 & 0,418 & 0,047876 \\
0,060 & 0,038074 & 0,099 & 0,023141 & 0,062 & 0,038708 & 0,068 & 0,019113 \\
0,403 & 0,099260 & 0,190 & 0,032185 & 0,092 & 0,047209 & 0,121 & 0,025628 \\
0,170 & 0,064296 & 0,188 & 0,032020 & 0,068 & 0,040549 & 0,106 & 0,023943 \\
0,138 & 0,057892 & 0,127 & 0,026220 & 0,114 & 0,052587 & 0,130 & 0,026565 \\
0,123 & 0,054636 & 0,115 & 0,024981 & 0,040 & 0,031049 & 0,049 & 0,016176 \\
0,145 & 0,059351 & 0,080 & 0,020796 & 0,481 & 0,108501 & 0,655 & 0,060071 \\
0,080 & 0,044004 & 0,088 & 0,021798 & 0,040 & 0,031049 & 0,063 & 0,018403 \\
0,151 & 0,060574 & 0,230 & 0,035404 & 0,200 & 0,069774 & 0,146 & 0,028214 \\
0,298 & 0,085275 & 0,286 & 0,039547 & 0,593 & 0,120551 & 0,609 & 0,057929 \\
2,644 & 0,255732 & 0,985 & 0,073824 & 0,163 & 0,062950 & 0,214 & 0,034134 \\
\hline
\end{tabular}


Tabela 6.7. Dados do exemplo 4 em Galea-Rojas et. al. (2003).

(Continuação...).

\begin{tabular}{|c|c|c|c|c|c|c|c|}
\hline $\mathrm{W}$ & $\sigma_{u}^{2}$ & $\mathrm{Y}$ & $\sigma_{e}^{2}$ & $\mathrm{~W}$ & $\sigma_{u}^{2}$ & $\mathrm{Y}$ & $\sigma_{e}^{2}$ \\
\hline 0,040 & 0,031049 & 0,040 & 0,014657 & 0,074 & 0,042311 & 0,141 & 0,027655 \\
0,040 & 0,031049 & 0,040 & 0,014657 & 0,764 & 0,136940 & 0,687 & 0,061558 \\
0,040 & 0,031049 & 0,063 & 0,018431 & 0,040 & 0,031049 & 0,190 & 0,032216 \\
0,235 & 0,075671 & 0,391 & 0,046312 & 0,040 & 0,031049 & 0,039 & 0,014466 \\
0,320 & 0,088387 & 0,731 & 0,063508 & 0,175 & 0,065241 & 0,163 & 0,029767 \\
0,115 & 0,052818 & 0,204 & 0,033321 & 0,192 & 0,068356 & 0,206 & 0,033515 \\
0,423 & 0,101709 & 0,345 & 0,043496 & 1,136 & 0,167189 & 0,844 & 0,068302 \\
0,101 & 0,049479 & 0,115 & 0,024995 & 0,133 & 0,056827 & 0,144 & 0,027929 \\
0,040 & 0,031049 & 0,201 & 0,033146 & 0,399 & 0,098763 & 0,279 & 0,039043 \\
1,373 & 0,183911 & 1,276 & 0,084134 & 0,071 & 0,041439 & 0,151 & 0,028701 \\
0,071 & 0,041439 & 0,221 & 0,034700 & 0,082 & 0,044554 & 0,109 & 0,024284 \\
1,132 & 0,166892 & 1,566 & 0,093312 & 0,175 & 0,065241 & 0,315 & 0,041509 \\
0,241 & 0,076637 & 0,380 & 0,045650 & 0,446 & 0,104454 & 0,286 & 0,039576 \\
0,306 & 0,086420 & 0,336 & 0,042899 & 0,203 & 0,070299 & 0,249 & 0,036890 \\
0,191 & 0,068176 & 0,272 & 0,038584 & 0,540 & 0,115004 & 0,494 & 0,052112 \\
0,265 & 0,080386 & 0,313 & 0,041400 & 4,523 & 0,335036 & 2,506 & 0,118282 \\
1,036 & 0,159615 & 0,971 & 0,073293 & 0,179 & 0,065987 & 0,479 & 0,051340 \\
0,065 & 0,039639 & 0,114 & 0,024895 & 0,167 & 0,063723 & 0,134 & 0,026959 \\
0,040 & 0,031049 & 0,060 & 0,017923 & 0,125 & 0,055081 & 0,174 & 0,030816 \\
0,040 & 0,031049 & 0,072 & 0,019729 & 0,336 & 0,090583 & 0,299 & 0,040435 \\
0,040 & 0,031049 & 0,066 & 0,018912 & 0,140 & 0,058313 & 0,227 & 0,035175 \\
0,250 & 0,078064 & 0,373 & 0,045221 & 0,040 & 0,031049 & 0,039 & 0,014494 \\
0,597 & 0,120959 & 0,343 & 0,043347 & 0,114 & 0,052587 & 0,098 & 0,023050 \\
0,129 & 0,055961 & 0,130 & 0,026595 & 0,040 & 0,031049 & 0,039 & 0,014465 \\
0,138 & 0,057892 & 0,165 & 0,029971 & 0,190 & 0,067997 & 0,186 & 0,031855 \\
\hline
\end{tabular}


Tabela 6.8. Dados do exemplo 4 em Galea-Rojas et. al. (2003).

(Continuação...).

\begin{tabular}{|c|c|c|c|c|c|c|c|}
\hline $\mathrm{W}$ & $\sigma_{u}^{2}$ & $\mathrm{Y}$ & $\sigma_{e}^{2}$ & $\mathrm{~W}$ & $\sigma_{u}^{2}$ & $\mathrm{Y}$ & $\sigma_{e}^{2}$ \\
\hline 0,139 & 0,058103 & 0,265 & 0,038090 & 0,253 & 0,078534 & 0,370 & 0,045058 \\
0,094 & 0,047723 & 0,140 & 0,027542 & 0,040 & 0,031049 & 0,039 & 0,014464 \\
0,130 & 0,056179 & 0,215 & 0,034276 & 0,364 & 0,094305 & 0,426 & 0,048365 \\
0,367 & 0,094696 & 0,425 & 0,048299 & 0,169 & 0,064106 & 0,144 & 0,027987 \\
0,127 & 0,055523 & 0,125 & 0,026037 & 0,479 & 0,108274 & 0,571 & 0,056093 \\
0,525 & 0,113386 & 0,588 & 0,056908 & 0,707 & 0,131701 & 0,634 & 0,059110 \\
0,668 & 0,127994 & 0,434 & 0,048830 & 0,735 & 0,134300 & 0,491 & 0,051949 \\
0,092 & 0,047209 & 0,143 & 0,027865 & 0,214 & 0,072190 & 0,149 & 0,028496 \\
0,107 & 0,050936 & 0,148 & 0,028410 & 0,040 & 0,031049 & 0,039 & 0,014417 \\
0,139 & 0,058103 & 0,120 & 0,025508 & 0,408 & 0,099878 & 0,487 & 0,051728 \\
0,248 & 0,077749 & 0,469 & 0,050791 & 0,116 & 0,053049 & 0,121 & 0,025652 \\
0,674 & 0,128572 & 0,372 & 0,045198 & 1,424 & 0,187317 & 1,677 & 0,096573 \\
0,394 & 0,098139 & 0,607 & 0,057837 & 0,798 & 0,139973 & 0,399 & 0,046777 \\
1,133 & 0,166966 & 1,154 & 0,079995 & 0,143 & 0,058938 & 0,269 & 0,038331 \\
0,701 & 0,131137 & 1,035 & 0,075712 & 0,354 & 0,092993 & 0,862 & 0,069042 \\
0,179 & 0,065987 & 0,158 & 0,029271 & 0,318 & 0,088108 & 0,383 & 0,045819 \\
0,106 & 0,050696 & 0,159 & 0,029419 & 0,231 & 0,075020 & 0,154 & 0,028926 \\
0,667 & 0,127898 & 0,616 & 0,058283 & 0,496 & 0,110190 & 0,485 & 0,051651 \\
1,395 & 0,185388 & 0,359 & 0,044342 & 1,444 & 0,188636 & 1,352 & 0,086634 \\
0,275 & 0,081898 & 0,199 & 0,032930 & 0,329 & 0,089629 & 0,257 & 0,037485 \\
0,454 & 0,105393 & 0,263 & 0,037890 & 0,249 & 0,077907 & 0,295 & 0,040201 \\
0,563 & 0,117443 & 0,790 & 0,066043 & 0,117 & 0,053278 & 0,067 & 0,018957 \\
0,188 & 0,067636 & 0,218 & 0,034495 & 0,194 & 0,068713 & 0,215 & 0,034239 \\
0,134 & 0,057042 & 0,162 & 0,029665 & 0,199 & 0,069598 & 0,210 & 0,033829 \\
0,246 & 0,077433 & 0,215 & 0,034217 & 0,407 & 0,099755 & 0,787 & 0,065918 \\
\hline
\end{tabular}


Tabela 6.9. Dados do exemplo 4 em Galea-Rojas et. al. (2003).

(Continuação...).

\begin{tabular}{|c|c|c|c|c|c|c|c|}
\hline $\mathrm{W}$ & $\sigma_{u}^{2}$ & $\mathrm{Y}$ & $\sigma_{e}^{2}$ & $\mathrm{~W}$ & $\sigma_{u}^{2}$ & $\mathrm{Y}$ & $\sigma_{e}^{2}$ \\
\hline 0,553 & 0,116389 & 0,669 & 0,060717 & 0,063 & 0,039021 & 0,040 & 0,014657 \\
1,014 & 0,157901 & 0,761 & 0,064832 & 0,274 & 0,081748 & 0,427 & 0,048404 \\
0,175 & 0,065241 & 0,154 & 0,028959 & 0,125 & 0,055081 & 0,125 & 0,026013 \\
0,117 & 0,053278 & 0,130 & 0,026570 & 0,259 & 0,079465 & 0,194 & 0,032518 \\
0,122 & 0,054412 & 0,118 & 0,025263 & 0,284 & 0,083236 & 0,315 & 0,041553 \\
0,260 & 0,079619 & 0,412 & 0,047540 & 0,086 & 0,045634 & 0,165 & 0,029936 \\
0,387 & 0,097258 & 0,313 & 0,041377 & 0,040 & 0,031049 & 0,109 & 0,024271 \\
2,872 & 0,266599 & 2,650 & 0,121669 & 0,092 & 0,047209 & 0,040 & 0,014657 \\
0,280 & 0,082644 & 0,223 & 0,034907 & 0,040 & 0,031049 & 0,048 & 0,016125 \\
0,311 & 0,087127 & 0,430 & 0,048608 & 0,478 & 0,108160 & 0,040 & 0,014657 \\
0,040 & 0,031049 & 0,039 & 0,014529 & 0,040 & 0,031049 & 0,039 & 0,014445 \\
0,799 & 0,140061 & 0,855 & 0,068762 & 0,254 & 0,078690 & 0,305 & 0,040830 \\
0,146 & 0,059557 & 0,146 & 0,028160 & 0,163 & 0,062950 & 0,096 & 0,022850 \\
0,104 & 0,050213 & 0,098 & 0,023029 & 0,345 & 0,091796 & 0,480 & 0,051365 \\
0,632 & 0,124476 & 1,071 & 0,077006 & 0,672 & 0,128379 & 0,651 & 0,059896 \\
0,230 & 0,074857 & 0,227 & 0,035162 & 0,945 & 0,152400 & 0,857 & 0,068822 \\
0,060 & 0,038074 & 0,038 & 0,014363 & 0,061 & 0,038392 & 0,064 & 0,018540 \\
0,040 & 0,031049 & 0,039 & 0,014453 & 0,199 & 0,069598 & 0,186 & 0,031847 \\
0,630 & 0,124278 & 0,233 & 0,035679 & 1,122 & 0,166149 & 1,702 & 0,097317 \\
0,406 & 0,099631 & 0,271 & 0,038507 & 0,073 & 0,042023 & 0,072 & 0,019740 \\
1,510 & 0,192925 & 1,675 & 0,096517 & 0,097 & 0,048483 & 0,129 & 0,026500 \\
0,107 & 0,050936 & 0,082 & 0,021047 & 0,310 & 0,086986 & 0,297 & 0,040306 \\
1,793 & 0,210340 & 0,995 & 0,074213 & 0,088 & 0,046165 & 0,086 & 0,021505 \\
0,064 & 0,039331 & 0,040 & 0,014573 & 0,201 & 0,069949 & 0,219 & 0,034585 \\
0,097 & 0,048483 & 0,101 & 0,023402 & 0,161 & 0,062560 & 0,126 & 0,026196 \\
\hline
\end{tabular}


Tabela 6.10. Dados do exemplo 4 em Galea-Rojas et. al. (2003).

(Continuação...).

\begin{tabular}{|c|c|c|c|c|c|c|c|}
\hline $\mathrm{W}$ & $\sigma_{u}^{2}$ & $\mathrm{Y}$ & $\sigma_{e}^{2}$ & $\mathrm{~W}$ & $\sigma_{u}^{2}$ & $\mathrm{Y}$ & $\sigma_{e}^{2}$ \\
\hline 0,040 & 0,031049 & 0,124 & 0,025965 & 0,073 & 0,042023 & 0,048 & 0,016130 \\
0,152 & 0,060776 & 0,218 & 0,034485 & 0,099 & 0,048984 & 0,090 & 0,022049 \\
0,199 & 0,069598 & 0,200 & 0,033019 & 0,070 & 0,041145 & 0,105 & 0,023888 \\
0,425 & 0,101950 & 0,557 & 0,055357 & 0,256 & 0,079001 & 0,372 & 0,045160 \\
0,116 & 0,053049 & 0,158 & 0,029342 & 0,507 & 0,111413 & 0,595 & 0,057228 \\
0,137 & 0,057681 & 0,119 & 0,025383 & 0,295 & 0,084843 & 0,460 & 0,050266 \\
0,040 & 0,031049 & 0,040 & 0,014565 & 0,140 & 0,058313 & 0,107 & 0,024102 \\
4,401 & 0,330458 & 2,215 & 0,111152 & 0,234 & 0,075509 & 0,331 & 0,042565 \\
0,381 & 0,096496 & 0,610 & 0,057950 & 0,078 & 0,043447 & 0,059 & 0,017814 \\
0,243 & 0,076956 & 0,267 & 0,038223 & 0,040 & 0,031049 & 0,054 & 0,017082 \\
0,067 & 0,040248 & 0,082 & 0,021015 & 0,040 & 0,031049 & 0,061 & 0,018198 \\
0,097 & 0,048483 & 0,092 & 0,022276 & 0,294 & 0,084698 & 0,382 & 0,045761 \\
0,093 & 0,047467 & 0,106 & 0,023950 & 0,193 & 0,068535 & 0,133 & 0,026860 \\
0,093 & 0,047467 & 0,074 & 0,020027 & 0,072 & 0,041732 & 0,174 & 0,030824 \\
0,040 & 0,031049 & 0,040 & 0,014647 & 0,461 & 0,106207 & 0,175 & 0,030894 \\
0,184 & 0,066908 & 0,133 & 0,026890 & 0,040 & 0,031049 & 0,040 & 0,014646 \\
0,814 & 0,141378 & 0,957 & 0,072752 & 0,113 & 0,052354 & 0,141 & 0,027713 \\
0,497 & 0,110302 & 0,479 & 0,051320 & 0,104 & 0,050213 & 0,109 & 0,024365 \\
0,512 & 0,111964 & 0,519 & 0,053457 & 0,072 & 0,041732 & 0,071 & 0,019599 \\
0,265 & 0,080386 & 0,291 & 0,039888 & 0,141 & 0,058522 & 0,163 & 0,029817 \\
0,071 & 0,041439 & 0,040 & 0,014607 & 0,040 & 0,031049 & 0,085 & 0,021399 \\
0,040 & 0,031049 & 0,040 & 0,014593 & 0,079 & 0,043726 & 0,081 & 0,020963 \\
0,176 & 0,065428 & 0,261 & 0,037789 & 0,190 & 0,067997 & 0,254 & 0,037222 \\
0,148 & 0,059966 & 0,163 & 0,029805 & 0,099 & 0,048984 & 0,108 & 0,024150 \\
0,060 & 0,038074 & 0,051 & 0,016582 & 0,138 & 0,057892 & 0,125 & 0,026022 \\
\hline
\end{tabular}


Tabela 6.11. Dados do exemplo 4 em Galea-Rojas et. al. (2003).

(Continuação...).

\begin{tabular}{|c|c|c|c|c|c|c|c|}
\hline $\mathrm{W}$ & $\sigma_{u}^{2}$ & $\mathrm{Y}$ & $\sigma_{e}^{2}$ & $\mathrm{~W}$ & $\sigma_{u}^{2}$ & $\mathrm{Y}$ & $\sigma_{e}^{2}$ \\
\hline 0,071 & 0,041439 & 0,073 & 0,019877 & 0,040 & 0,031049 & 0,046 & 0,015759 \\
0,107 & 0,050936 & 0,106 & 0,023982 & 0,103 & 0,049969 & 0,095 & 0,022652 \\
0,040 & 0,031049 & 0,066 & 0,018822 & 0,303 & 0,085992 & 0,287 & 0,039612 \\
0,080 & 0,044004 & 0,104 & 0,023713 & 0,152 & 0,060776 & 0,156 & 0,029155 \\
0,090 & 0,046690 & 0,105 & 0,023909 & 0,122 & 0,054412 & 0,124 & 0,025937 \\
0,113 & 0,052354 & 0,064 & 0,018643 & 0,084 & 0,045097 & 0,083 & 0,021203 \\
0,059 & 0,037754 & 0,079 & 0,020649 & 0,092 & 0,047209 & 0,081 & 0,020896 \\
0,431 & 0,102672 & 0,588 & 0,056900 & 0,072 & 0,041732 & 0,070 & 0,019480 \\
0,111 & 0,051886 & 0,136 & 0,027182 & 0,040 & 0,031049 & 0,056 & 0,017427 \\
0,040 & 0,031049 & 0,040 & 0,014615 & 1,835 & 0,212805 & 1,290 & 0,084605 \\
0,071 & 0,041439 & 0,079 & 0,020708 & 0,040 & 0,031049 & 0,053 & 0,016903 \\
0,094 & 0,047723 & 0,121 & 0,025583 & 0,405 & 0,099508 & 0,292 & 0,040001 \\
0,417 & 0,100980 & 0,630 & 0,058939 & 0,284 & 0,083236 & 0,266 & 0,038139 \\
0,040 & 0,031049 & 0,048 & 0,016080 & 0,130 & 0,056179 & 0,097 & 0,022903 \\
0,476 & 0,107932 & 0,366 & 0,044812 & 0,201 & 0,069949 & 0,176 & 0,030944 \\
0,040 & 0,031049 & 0,049 & 0,016184 & 0,113 & 0,052354 & 0,091 & 0,022190 \\
0,040 & 0,031049 & 0,047 & 0,015976 & 0,194 & 0,068713 & 0,534 & 0,054222 \\
1,147 & 0,168001 & 0,926 & 0,071560 & 0,329 & 0,089629 & 0,357 & 0,044258 \\
0,040 & 0,031049 & 0,040 & 0,014591 & 0,079 & 0,043726 & 0,074 & 0,020025 \\
1,569 & 0,196682 & 1,027 & 0,075418 & 0,074 & 0,042311 & 0,063 & 0,018419 \\
0,246 & 0,077433 & 0,186 & 0,031829 & 0,103 & 0,049969 & 0,101 & 0,023398 \\
0,040 & 0,031049 & 0,039 & 0,014533 & 0,201 & 0,069949 & 0,238 & 0,036048 \\
0,843 & 0,143890 & 0,437 & 0,049005 & 0,237 & 0,075994 & 0,239 & 0,036093 \\
0,129 & 0,055961 & 0,109 & 0,024296 & 0,083 & 0,044826 & 0,077 & 0,020379 \\
0,172 & 0,064676 & 0,100 & 0,023331 & 0,138 & 0,057892 & 0,177 & 0,031086 \\
\hline
\end{tabular}


Tabela 6.12. Dados do exemplo 4 em Galea-Rojas et. al. (2003).

(Continuação...).

\begin{tabular}{|c|c|c|c|c|c|c|c|}
\hline $\mathrm{W}$ & $\sigma_{u}^{2}$ & $\mathrm{Y}$ & $\sigma_{e}^{2}$ & $\mathrm{~W}$ & $\sigma_{u}^{2}$ & $\mathrm{Y}$ & $\sigma_{e}^{2}$ \\
\hline 0,084 & 0,045097 & 0,058 & 0,017752 & 0,451 & 0,105042 & 0,299 & 0,040441 \\
0,259 & 0,079465 & 0,162 & 0,029732 & 0,309 & 0,086845 & 0,229 & 0,035384 \\
0,068 & 0,040549 & 0,055 & 0,017282 & 0,040 & 0,031049 & 0,071 & 0,019646 \\
0,040 & 0,031049 & 0,039 & 0,014474 & 0,075 & 0,042598 & 0,100 & 0,023250 \\
0,040 & 0,031049 & 0,039 & 0,014425 & 0,113 & 0,052354 & 0,174 & 0,030801 \\
0,081 & 0,044280 & 0,076 & 0,020289 & 0,082 & 0,044554 & 0,131 & 0,026689 \\
0,040 & 0,031049 & 0,039 & 0,014490 & 0,209 & 0,071336 & 0,352 & 0,043897 \\
0,116 & 0,053049 & 0,156 & 0,029157 & 0,125 & 0,055081 & 0,178 & 0,031153 \\
0,092 & 0,047209 & 0,105 & 0,023873 & 0,066 & 0,039945 & 0,081 & 0,020959 \\
0,092 & 0,047209 & 0,150 & 0,028589 & 0,849 & 0,144404 & 0,558 & 0,055401 \\
0,142 & 0,058730 & 0,085 & 0,021494 & 0,477 & 0,108046 & 0,275 & 0,038802 \\
0,103 & 0,049969 & 0,202 & 0,033177 & 0,040 & 0,031049 & 0,039 & 0,014470 \\
0,103 & 0,049969 & 0,127 & 0,026298 & 0,341 & 0,091259 & 0,182 & 0,031498 \\
0,165 & 0,063338 & 0,152 & 0,028777 & 0,107 & 0,050936 & 0,231 & 0,035524 \\
0,098 & 0,048734 & 0,108 & 0,024160 & 0,097 & 0,048483 & 0,118 & 0,025346 \\
0,093 & 0,047467 & 0,172 & 0,030619 & 0,161 & 0,062560 & 0,223 & 0,034915 \\
0,070 & 0,041145 & 0,044 & 0,015463 & 0,135 & 0,057255 & 0,157 & 0,029224 \\
0,071 & 0,041439 & 0,069 & 0,019231 & 0,647 & 0,125954 & 0,556 & 0,055342 \\
0,040 & 0,031049 & 0,067 & 0,019034 & 0,211 & 0,071679 & 0,199 & 0,032899 \\
0,062 & 0,038708 & 0,084 & 0,021338 & 0,245 & 0,077274 & 0,348 & 0,043664 \\
0,040 & 0,031049 & 0,047 & 0,015849 & 0,826 & 0,142423 & 1,092 & 0,077778 \\
0,439 & 0,103626 & 0,504 & 0,052666 & 0,322 & 0,088664 & 0,628 & 0,058841 \\
0,230 & 0,074857 & 0,295 & 0,040168 & 0,314 & 0,087549 & 0,401 & 0,046923 \\
0,305 & 0,086277 & 0,364 & 0,044675 & 0,577 & 0,118903 & 0,622 & 0,058546 \\
0,261 & 0,079773 & 0,287 & 0,039613 & 0,040 & 0,031049 & 1,270 & 0,083939 \\
0,215 & 0,072360 & 0,262 & 0,037843 & & & & \\
\hline
\end{tabular}




\section{BIBLIOGRAFIA}

*[1] Barnett, V.D., Simulation pairwise linear structural relationship, Biometrics, 25 (1969), 129-142.

$\star[2]$ Bolfarine, H. and Gimenez, P., Corrected score functions in classical error-invariables and incidentais parameters models. Australian Journal Statistical, 39, 3, (1997), 325-344.

*[3] Carroll, R.J., Ruppert, D. and Stefanski, L.A. Measurement Error in Nonlinear Models. Chapman and Hall, New York, (1995).

*[4] Castro, M., Castilho, M.V. and Bolfarine, H. Consistent Estimation and Testing in Comparing Analytical bias Models. Relatório técnico do Departamento de Estatística da Universidade de São Paulo (RT-MAE 2004-01) (2004).

$\star$ [5] Chan and Mak On the polynomial functional relationship J. R. Statist. Soc. B, 47 (3) (1985), 510-518.

*[6] Cheng, C-L. On the polynomial Berkson Model. Inst. of Statis. Science, Acad. Sinica, Taiwan - China, (1997).

*[7] Cheng, C-L. and Scheneeweiss, H. Polynomial Regression with error in the variables. J.R. Statist., B, 60, Part I, (1998), 189-199. 
*[8] Cheng, C-L. and Vann Ness, J. Statistical Regression with Measurement Error. Arnold. Oxford University, Press Inc., New York, (1999).

*[9] Cook, R. Assessment of local Influence. J.R. Statist. Soc., B, 48 (2), (1986), 133-169.

*[10] Cook, J. and Stefanski, L.A. A Simulation Extrapolation Method for parametric measurement error model. Journal of the American Statistical Asociation, 89, (1995), 1314-1328.

$\star$ «11] Fuller, W.A. Measurement Error Models. John Willey and Sons, New York, (1987).

$\star$ «12] Galea-Rojas, M., de Castilho, M., Bolfarine, H. and de Castro, M. Detection of analytical bias, The Analyst, (2003), p 1073-1081.

*[13] Gasco, L., Bolfarine, H. and Arellano-Valle, R. Measurement Error Models with Nonconstant Covariance Matrices. (2002), Vol 82, Issue 2, pp 395-415.

*[14] Hubert P.J., Robust Statistics. (1981). New York. Willey.

*[15] Hwang, J. Multiplicative error in variables models with applications to recent data released by U.S. Departament of Energy. Journal of the American Statistical Asociation, 81, (1986), 680-688.

$\star[16]$ Lee, A. and Zhao Y. Assessing local influence in measurement error models. Biometrical Journal, 38, 7,(1996), 829-841.

*[17] Nakamura, T. Corrected score function for errors-in-variables models: Methodology and application to generalized linear model. Biometrika, 77, 1,(1990), 127-137.

*[18] Stylan, G. Hadamard product and multivariate statistical analysis. Linear algebra and its applications, 6, (1973), 217-240.

$\star[19]$ Wolter, K. and Fuller W. Estimation of the quadratic error-in-variables model. Biometrika, 69, 1,(1982), 175-182. 